\title{
Titanocene-Catalyzed Reductive Domino Epoxide Ring Opening/Defluorinative Cross-Coupling Reaction
}

\author{
Zhiyang Lin, ${ }^{1}$ Yun Lan, ${ }^{1}$ and Chuan Wang ${ }^{*, 1,2}$ \\ ${ }^{1}$ Hefei National Laboratory for Physical Science at the Microscale and Department of Chemistry, \\ University of Science and Technology of China, Hefei, Anhui, 230026 (P. R. China). \\ ${ }^{2}$ Center for Excellence in Molecular Synthesis of CAS, Hefei, Anhui, 230026 (P. R. China). \\ E-mail: chuanw@ustc.edu.cn \\ Table of Contents
}

I. General Information. ...................................................................... 2

II. Preparation of Substrates ................................................................... 3

III. Standard Procedures for Synthesis of the gem-Difluoroalkenes 3............... 6

IV. Procedure for Synthesis of the 6-Fluoro-3,4-Dihydro-2H-Pyrans 4.............23

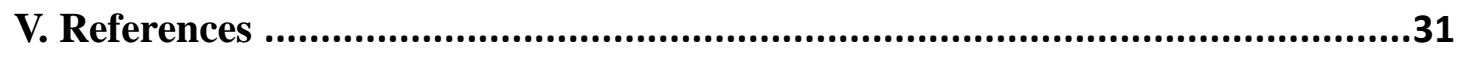

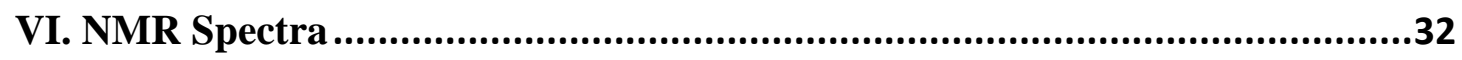

VII. HPLC Data and Chromatograms ....................................................193 


\section{General Information.}

${ }^{1} \mathrm{H}$ NMR, ${ }^{13} \mathrm{C}$ NMR and ${ }^{19} \mathrm{~F}$ NMR spectra were recorded on a Bruker Advance 400M NMR spectrometers at ambient temperature in $\mathrm{CDCl}_{3}$ at 400,101 and $376 \mathrm{MHz}$, respectively. ${ }^{19} \mathrm{~F}$ NMR were reported as ${ }^{19} \mathrm{~F}$ exp. comp. pulse decoupling $\left(\mathrm{F}^{19} \mathrm{CPD}\right.$ ) unless otherwise noted. The chemical shifts are given in ppm relative to tetramethylsilane $\left[{ }^{1} \mathrm{H}: \delta=\left(\mathrm{SiMe}_{4}\right)=0.00 \mathrm{ppm}\right]$ as an internal standard or relative to the resonance of the solvent $\left[{ }^{1} \mathrm{H}: \delta\left(\mathrm{CDCl}_{3}\right)=7.26,{ }^{13} \mathrm{C}: \delta\left(\mathrm{CDCl}_{3}\right)=77.16 \mathrm{ppm}\right]$. Multiplicities were given as: s (singlet); $\mathrm{d}$ (doublet); $\mathrm{t}$ (triplet); q (quartet); dd (doublet of doublets); dt (doublet of triplets); m (multiplets), etc. Coupling constants are reported as $J$ values in Hz. High resolution mass spectral analysis (HRMS) was performed on Waters XEVO G2 Q-TOF using ESI technique. Flash chromatography was performed using 300-400 mesh silica gel with the indicated solvent system.

$\mathrm{Cp}_{2} \mathrm{TiCl}_{2}$ and $\mathrm{NEt}_{3} \cdot \mathrm{HCl}$ were purchased from Adamas-beta. All solvents were purchased from Adamas-beta China and used as received. Other commercial reagents were purchased from Sigma-Aldrich, Alfa Aesar, TCI, Strem, Acros and Energy Chemical, and were used as received.

Reactions were monitored through thin layer chromatography [Merck 60 F254 precoated silica gel plate $(0.2 \mathrm{~mm}$ thickness $)]$. Subsequent to elution, spots were visualized using UV radiation (254 nm) on Spectroline Model ZF-7 $254 \mathrm{~nm}$. Other visualization methods include staining with a basic solution of potassium permanganate or acidic solution of ceric ammonium molybdate, followed by heating. 


\section{Preparation of Substrates}

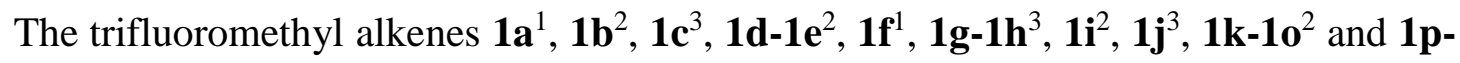
$\mathbf{1 \mathbf { q } ^ { 3 }}$ were synthesized following the reported procedures.

The epoxides $\mathbf{2 b}$ and $\mathbf{2 q}$ were commercially available.

The epoxides $2 \mathbf{a}^{4}, \mathbf{2} \mathbf{c}^{5}, \mathbf{2} \mathbf{d}-\mathbf{2} \mathbf{e}^{6}, \mathbf{2} \mathbf{f}^{7}, \mathbf{2} \mathbf{i}^{7}, \mathbf{2} \mathbf{k}^{8}, \mathbf{2} \mathbf{l}^{9}, \mathbf{2} \mathbf{m}^{10}$ and $\mathbf{2} \mathbf{p}^{11}$ were synthesized following the reported procedures.

\section{Procedure for Preparation of the Racemic Epoxides $2 \mathrm{~h}, 2 \mathrm{j}, 2 \mathrm{n}$ and $2 \mathrm{o}$}

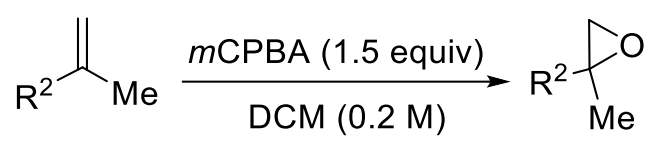

A flask charged with $m$ CPBA ( $3.0 \mathrm{~g}, 15 \mathrm{mmol}, 1.5$ equiv, $85 \%$ purity) in dry DCM (50 $\mathrm{mL}$ ) was cooled to $0{ }^{\circ} \mathrm{C}$, before the olefins ( $10 \mathrm{mmol}, 1.0$ equiv) were added to the flask. The resultant mixture was allowed to warm up and stir at room temperature for 10 hours. Then the reaction was quenched with saturated aq. $\mathrm{Na}_{2} \mathrm{SO}_{3}(25 \mathrm{~mL})$ and $\mathrm{Na}_{2} \mathrm{CO}_{3}(25$ $\mathrm{mL})$. The aqueous phase was extracted with DCM $(3 \times 25 \mathrm{~mL})$, and the combined organic phases were dried over $\mathrm{MgSO}_{4}$, filtered, and concentrated under reduced pressure. The crude product was purified through column chromatography on silica gel (petroleum ether/ethyl acetate) to give the epoxides.

2-(2-(4-(tert-Butyl)phenoxy)ethyl)-2-methyloxirane (2h)<smiles>CC1(CCOc2ccc(C(C)(C)C)cc2)CO1</smiles>

$2 \mathrm{~h}$

The title compound $\mathbf{2 h}$ was isolated as a colorless oil $(1.5 \mathrm{~g}, 65 \%)$ through flash chromatography on silica gel eluting with petroleum ether/ethyl acetate (5:1).

${ }^{1}$ H NMR (400 MHz, Chloroform-d) $\delta=7.29(\mathrm{~d}, J=8.8 \mathrm{~Hz}, 2 \mathrm{H}), 6.82(\mathrm{~d}, J=8.8 \mathrm{~Hz}$, $2 \mathrm{H}), 4.12-3.96(\mathrm{~m}, 2 \mathrm{H}), 2.73(\mathrm{~d}, J=4.8 \mathrm{~Hz}, 1 \mathrm{H}), 2.61(\mathrm{~d}, J=4.8 \mathrm{~Hz}, 1 \mathrm{H}), 2.15-1.94(\mathrm{~m}$, 2H), 1.39 (s, 3H), 1.30 (s, 9H).

${ }^{13}$ C NMR (101 MHz, Chloroform- $d$ ) $\delta=156.4,143.5,126.3$ (2C), 113.9 (2C), 64.2, 55.3, 54.0, 36.1, 34.1, 31.6 (3C), 21.7 ppm.

HRMS (ESI) m/z: [M+Na] ${ }^{+}$Calcd for $\mathrm{C}_{15} \mathrm{H}_{22} \mathrm{O}_{2} \mathrm{Na} 257.1512$; Found 257.1517 
<smiles>CC(=O)c1ccc(OCCC2(C)CO2)cc1</smiles>

2j

The title compound $\mathbf{2} \mathbf{j}$ was isolated as a colorless oil $(1.1 \mathrm{~g}, 45 \%)$ through flash chromatography on silica gel eluting with petroleum ether/ethyl acetate (4:1).

${ }^{1}$ H NMR (400 MHz, Chloroform-d) $\delta=7.92(\mathrm{~d}, J=9.0 \mathrm{~Hz}, 2 \mathrm{H}), 6.92(\mathrm{~d}, J=8.9 \mathrm{~Hz}$, $2 \mathrm{H}), 4.21-3.96(\mathrm{~m}, 2 \mathrm{H}), 2.7-2.71(\mathrm{~m}, 1 \mathrm{H}), 2.67-2.61(\mathrm{~m}, 1 \mathrm{H}), 2.54(\mathrm{~s}, 3 \mathrm{H}), 2.14-2.03$ (m, 2H), 1.41 (s, 3H) ppm.

${ }^{13}$ C NMR (101 MHz, Chloroform-d) $\delta=196.7,162.5,130.6$ (2C), 130.3, 114.1 (2C), 64.5, 55.0, 53.9, 35.8, 26.3, $21.6 \mathrm{ppm}$.

HRMS (ESI) m/z: [M+H] $]^{+}$Calcd for $\mathrm{C}_{13} \mathrm{H}_{17} \mathrm{O}_{3}$ 221.1172; Found 221.1170

2-(2-Methyloxiran-2-yl)ethyl 3-cyclohexylpropanoate (2n)<smiles>CC1(CCOC(=O)CCC2CCCCC2)CO1</smiles>

The title compound $2 \mathrm{n}$ was isolated as a colorless oil (1.2 g, 52\%) through flash chromatography on silica gel eluting with petroleum ether/ethyl acetate $(7: 1)$.

${ }^{1}$ H NMR (400 MHz, Chloroform- $d$ ) $\delta=4.33-4.04$ (m, 2H), 2.72-2.48 (m, 2H), $2.31(\mathrm{t}$, $J=7.9 \mathrm{~Hz}, 2 \mathrm{H}), 2.01-1.89(\mathrm{~m}, 1 \mathrm{H}), 1.89-1.80(\mathrm{~m}, 1 \mathrm{H}), 1.75-1.60(\mathrm{~m}, 5 \mathrm{H}), 1.57-1.46(\mathrm{~m}$, 2H), 1.36 (s, 3H), 1.28-1.09 (m, 4H), 0.96-0.82 (m, 2H) ppm.

${ }^{13}$ C NMR (101 MHz, Chloroform-d) $\delta=173.9,60.7,54.9,53.6,37.1,35.6,32.9$ (2C), 32.3, 31.8, 26.5, $26.2(2 \mathrm{C}), 21.2 \mathrm{ppm}$.

HRMS (ESI) m/z: [M+Na] ${ }^{+}$Calcd for $\mathrm{C}_{14} \mathrm{H}_{24} \mathrm{O}_{3} \mathrm{Na}$ 263.1618; Found 263.1620

2-(2-(2-Methyloxiran-2-yl)ethyl)isoindoline-1,3-dione (2o)<smiles>CC1(CCN2C(=O)c3ccccc3C2=O)CO1</smiles>

The title compound 20 was isolated as a colorless oil $(1.4 \mathrm{~g}, 62 \%)$ through flash chromatography on silica gel eluting with petroleum ether/ethyl acetate $(2: 1)$.

${ }^{1}$ H NMR (400 MHz, Chloroform- $d$ ) $\delta=7.85(\mathrm{dd}, J=5.4,3.0 \mathrm{~Hz}, 2 \mathrm{H}), 7.72$ (dd, $J=5.5$, $3.1 \mathrm{~Hz}, 2 \mathrm{H}), 3.94-3.74(\mathrm{~m}, 2 \mathrm{H}), 2.60(\mathrm{~d}, J=4.7 \mathrm{~Hz}, 1 \mathrm{H}), 2.54$ (d, J=4.7 Hz, 1H), 2.05$1.87(\mathrm{~m}, 2 \mathrm{H}), 1.43(\mathrm{~s}, 3 \mathrm{H}) \mathrm{ppm}$. 
${ }^{13}$ C NMR (101 MHz, Chloroform-d) $\delta=168.2$ (2C), 133.9 (2C), 132.1 (2C), 123.3 (2C), 55.2, 53.2, 35.0, 34.4, 20.7 ppm.

HRMS (ESI) m/z: [M+Na $]^{+}$Calcd for $\mathrm{C}_{13} \mathrm{H}_{13} \mathrm{NO}_{3} \mathrm{Na} 254.0788$; Found 254.0792

\section{Procedure for Preparation of the Enantioenriched Epoxide 2g}

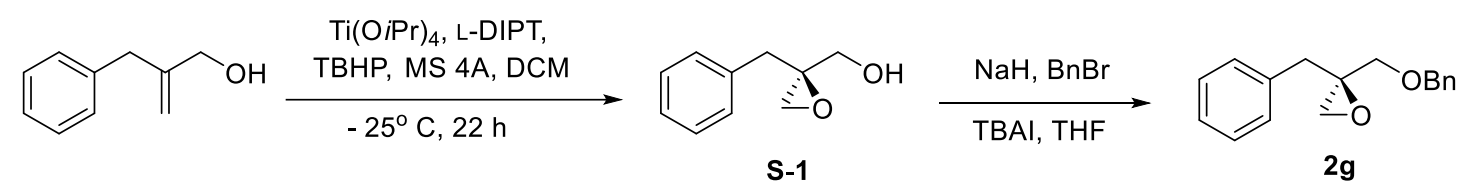

Step 1:12 A flame-dried round-bottom flask equipped with a magnetic stirring bar was charged with activated molecular sieves $4 \AA(1.0 \mathrm{~g})$. The flask was evacuated and refilled with nitrogen. To the flask were added $\mathrm{CH}_{2} \mathrm{Cl}_{2}(36 \mathrm{~mL}), \mathrm{Ti}(\mathrm{O} i \mathrm{Pr})_{4}(231 \mu \mathrm{L}$, $0.75 \mathrm{mmol}, 0.15$ equiv), and L-DIPT ( $315 \mu \mathrm{L}, 1.50 \mathrm{mmol}, 0.3$ equiv). After cooling to $-25^{\circ} \mathrm{C}$, a solution of TBHP in $n$-decane $(5.5 \mathrm{M}, 1.8 \mathrm{~mL}, 10 \mathrm{mmol}, 2.0$ equiv) was added, and the resulting mixture was stirred at the same temperature for $1 \mathrm{~h}$. Then, $(S)$ - $(2-$ benzyloxiran-2-yl)methanol ( $820 \mathrm{mg}, 5 \mathrm{mmol}, 1.0$ equiv) dissolved in $\mathrm{CH}_{2} \mathrm{Cl}_{2}(8.0 \mathrm{~mL})$ was added over $10 \mathrm{~min}$ via a syringe. After stirring for $22 \mathrm{~h}, 3 \mathrm{M}$ aq. $\mathrm{NaOH}(2.0 \mathrm{~mL})$ was added, and the reaction mixture was stirred for $3 \mathrm{~h}$ (During this time L-DIPT was converted to tartaric acid). After filtration through Celite, the organic layer was separated, and the aqueous layer was extracted with $\mathrm{Et}_{2} \mathrm{O}$. The combined organic layer was washed with brine and dried over $\mathrm{Na}_{2} \mathrm{SO}_{4}$. After filtration, the volatiles were removed under reduced pressure, and the resulting residue was purified by silica gel column chromatography (petroleum ether/ethyl acetate $=3: 1$ ) to afford epoxide $\mathbf{S}-\mathbf{1}$ as a colorless oil (648 mg, 79\%). ${ }^{1} \mathbf{H}$ NMR (500 MHz, Chloroform- $d$ ) $\delta=7.33-7.26(\mathrm{~m}$, $2 \mathrm{H}), 7.26-7.20(\mathrm{~m}, 3 \mathrm{H}), 3.72(\mathrm{dd}, J=12.4,4.5 \mathrm{~Hz}, 1 \mathrm{H}), 3.57(\mathrm{dd}, J=12.4,7.8 \mathrm{~Hz}, 1 \mathrm{H})$, $3.07(\mathrm{~d}, J=14.3 \mathrm{~Hz}, 1 \mathrm{H}), 2.87(\mathrm{~s}, 1 \mathrm{H}), 2.85(\mathrm{~d}, J=11.2 \mathrm{~Hz}, 1 \mathrm{H}), 2.66(\mathrm{~d}, J=4.7 \mathrm{~Hz}$, $1 \mathrm{H}), 2.19$ (dd, $J=8.0,5.0 \mathrm{~Hz}, 1 \mathrm{H})$ ppm. ${ }^{13} \mathrm{C}$ NMR (126 MHz, Chloroform-d) $\delta=136.1$, $129.6(2 \mathrm{C}), 128.5$ (2C), 126.9, 62.8, 60.2, 49.7, 38.3 ppm.

Step 2: To the suspension of sodium hydride (52 $\mathrm{mg}$ as a $60 \%$ dispersion in mineral oil, $1.3 \mathrm{mmol}, 1.3$ equiv. $)$ in THF $(1 \mathrm{~mL})$ was added a solution of the $(S)$-(2-benzyloxiran2-yl)methanol S-1 (164 mg, $1.0 \mathrm{mmol}, 1.0$ equiv) in THF (2 mL) at $-78{ }^{\circ} \mathrm{C}$. After stirring for $20 \mathrm{~min}$, a solution of benzyl bromide ( $257 \mathrm{mg}, 1.5 \mathrm{mmol}, 1.5 \mathrm{equiv}$ ) in THF ( $2 \mathrm{~mL}$ ) and tetrabutylammonium iodide ( $185 \mathrm{mg}, 0.5 \mathrm{mmol}, 0.5$ equiv.) were added to reaction mixture, sequentially. The reaction was warmed to room temperature and stirred for $6 \mathrm{~h}$. Then the reaction was quenched with saturated aq. $\mathrm{NH}_{4} \mathrm{Cl}(5 \mathrm{~mL})$. The aqueous phase was extracted with EA $(3 \times 10 \mathrm{~mL})$, and the combined organic phases were dried over $\mathrm{Na}_{2} \mathrm{SO}_{4}$, filtered, and concentrated under reduced pressure. The crude product was purified through column chromatography (petroleum ether/ethyl acetate $=$ 5:1), the epoxide (R)-2-benzyl-2-((benzyloxy)methyl)oxirane (2g) was obtained as a colorless oil in $80 \%$ yield $(203 \mathrm{mg}, 82 \% e e$ ). The $e e$ was determined by HPLC analysis (Chiral IA column, $\lambda=254 \mathrm{~nm}$, hexane/isopropanol $=95 / 5$, flow rate $=0.5 \mathrm{~mL} / \mathrm{min}$ ): $\mathrm{t}_{\mathrm{R}}($ minor $)=8.9 \mathrm{~min}, \mathrm{t}_{\mathrm{R}}($ major $)=9.5 \mathrm{~min}$. 
${ }^{1}$ H NMR (500 MHz, Chloroform-d) $\delta=7.34(\mathrm{~m}, 4 \mathrm{H})$, 7.32-7.24 (m, 3H), 7.26-7.19 $(\mathrm{m}, 3 \mathrm{H}), 4.57(\mathrm{~d}, J=11.9 \mathrm{~Hz}, 1 \mathrm{H}), 4.51(\mathrm{~d}, J=11.9 \mathrm{~Hz}, 1 \mathrm{H}), 3.52(\mathrm{~d}, J=10.9 \mathrm{~Hz}, 1 \mathrm{H})$, 3.40 (d, $J=10.9 \mathrm{~Hz}, 1 \mathrm{H}), 3.13$ (d, $J=14.2 \mathrm{~Hz}, 1 \mathrm{H}), 2.90$ (d, $J=14.2 \mathrm{~Hz}, 1 \mathrm{H}), 2.71$ (d, $J=4.8 \mathrm{~Hz}, 1 \mathrm{H}), 2.64(\mathrm{~d}, J=4.7 \mathrm{~Hz}, 1 \mathrm{H}) \mathrm{ppm}$.

${ }^{13}$ C NMR (126 MHz, Chloroform-d) $\delta=137.9,136.5,129.8$ (2C), 128.5 (2C), 128.4 (2C), 127.8 (2C), 127.8, 126.7, 73.3, 71.6, 59.0, 50.1, 38.3 ppm.

HRMS (ESI) $\mathrm{m} / \mathrm{z}$ : $[\mathrm{M}+\mathrm{H}]^{+}$Calcd for $\mathrm{C}_{17} \mathrm{H}_{19} \mathrm{O}_{2} 255.1380$; Found 255.1384

\section{Standard Procedures for Synthesis of the gem-Difluoro -}

\section{alkenes 3.}

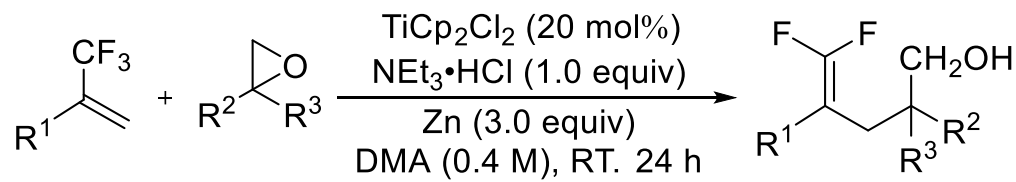

$\mathrm{TiCp}_{2} \mathrm{Cl}_{2}(20 \mathrm{mg}, 0.08 \mathrm{mmol}, 20 \mathrm{~mol} \%), \mathrm{NEt}_{3} \cdot \mathrm{HCl}(55 \mathrm{mg}, 0.4 \mathrm{mmol}, 1.0$ equiv) and $\mathrm{Zn}$ (78 mg, $1.2 \mathrm{mmol}, 3$ equiv) were placed in a Schlenk tube equipped with a stir bar. Then the Schlenk tube was evacuated and filled with nitrogen (three cycles). To these solids, $1.0 \mathrm{~mL}$ dry DMA, trifluoromethyl alkenes $1(0.4 \mathrm{mmol}, 1.0 \text { equiv })^{\mathrm{a}}$ and epoxides 2 ( 0.8 mmol, 2.0 equiv) were added successively under nitrogen atmosphere. The reaction mixture was stirred at room temperature for 24 hours, before it was quenched by addition of water. The aqueous phase was extracted with ethyl acetate $(3 \times 20 \mathrm{~mL})$, and the combined organic phases were washed with brine, dried over $\mathrm{Na}_{2} \mathrm{SO}_{4}$, filtered and concentrated in vacuo. The crude material was then purified through column chromatography on silica gel (petroleum ether/ethyl acetate) to give the gemdifluoroalkenes $\mathbf{3}$ as products.

${ }^{a} 0.2 \mathrm{mmol}$ for $\mathbf{1 q}$.

5,5-Difluoro-2-(4-methoxyphenethyl)-4-(4-methoxyphenyl)-2-methylpent-4-en-1-ol (3aa)<smiles>COc1ccc(CCC(C)(CO)CC(=C(F)F)c2ccc(OC)cc2)cc1</smiles>

The title compound 3aa was isolated as a colorless oil (122 mg, 81\%) through flash chromatography on silica gel eluting with petroleum ether/ethyl acetate (3:1). 
${ }^{1}$ H NMR (400 MHz, Chloroform-d) $\delta=7.25(\mathrm{~d}, J=8.8 \mathrm{~Hz}, 2 \mathrm{H}), 6.94(\mathrm{~d}, J=8.6 \mathrm{~Hz}$, 2H), 6.88 (d, J=8.8 Hz, 2H), 6.77 (d, $J=8.6 \mathrm{~Hz}, 2 \mathrm{H}), 3.77$ (s, 3H), $3.74(\mathrm{~s}, 3 \mathrm{H}), 3.20$ (s, 2H), 2.51-2.35 (m, 4H), 1.52-1.42 (m, 2H), $0.83(\mathrm{~s}, 3 \mathrm{H}) \mathrm{ppm}$.

${ }^{13}$ C NMR (101 MHz, Chloroform-d) $\delta=158.8,157.7,154.4(\mathrm{dd}, J=289.1,287.1 \mathrm{~Hz})$, 134.9, 129.6 (t, $J=2.6 \mathrm{~Hz}, 2 \mathrm{C}), 129.2$ (2C), 127.2 (dd, $J=4.7,2.5 \mathrm{~Hz}), 114.1(2 \mathrm{C})$, 113.8 (2C), 89.6 (dd, $J=21.5,14.1 \mathrm{~Hz}), 68.7,55.27,55.25,39.9$ (t, $J=2.4 \mathrm{~Hz}$ ), 39.3, $34.3,29.1,21.8 \mathrm{ppm}$.

${ }^{19}$ F NMR (376 MHz, Chloroform- $d$ ) $\delta=-90.08(\mathrm{~d}, J=42.5 \mathrm{~Hz}, 1 \mathrm{~F}),-91.97$ (d, $J=42.9$ $\mathrm{Hz}, 1 \mathrm{~F}) \mathrm{ppm}$.

HRMS (ESI) m/z: [M+Na] $]^{+}$Calcd for $\mathrm{C}_{22} \mathrm{H}_{26} \mathrm{~F}_{2} \mathrm{O}_{3} \mathrm{Na}$ 399.1742; Found 399.1746

4-(3,5-Dimethoxyphenyl)-5,5-difluoro-2-(4-methoxyphenethyl)-2-methylpent-4-en-1ol (3ba)<smiles>COc1ccc(CCC(C)(CO)CC(=C(F)F)c2cc(OC)cc(OC)c2)cc1</smiles>

The title compound 3ba was isolated as a colorless oil (102 mg, 63\%) through flash chromatography on silica gel eluting with petroleum ether/ethyl acetate (3:1).

${ }^{1}$ H NMR (400 MHz, Chloroform-d) $\delta=6.97$ (d, $\left.J=8.6 \mathrm{~Hz}, 2 \mathrm{H}\right), 6.78(\mathrm{~d}, J=8.6 \mathrm{~Hz}$, $2 \mathrm{H}), 6.49(\mathrm{~s}, 2 \mathrm{H}), 6.39(\mathrm{~s}, 1 \mathrm{H}), 3.77(\mathrm{~s}, 6 \mathrm{H}), 3.76(\mathrm{~s}, 3 \mathrm{H}), 3.23(\mathrm{~s}, 2 \mathrm{H}), 2.48-2.41(\mathrm{~m}$, $4 \mathrm{H}), 1.55-1.44(\mathrm{~m}, 2 \mathrm{H}), 0.86(\mathrm{~s}, 3 \mathrm{H}) \mathrm{ppm}$.

${ }^{13}$ C NMR (101 MHz, Chloroform- $d$ ) $\delta=160.8$ (2C), 157.7, 154.4 (dd, $J=290.9,287.5$ $\mathrm{Hz}$ ), 137.1 (dd, $J=5.0,2.4 \mathrm{~Hz}), 134.9,129.1$ (2C), 113.7 (2C), 106.8 (t, $J=2.9 \mathrm{~Hz}, 2 \mathrm{C}$ ), 99.2, 90.2 (dd, $J=21.9,13.4 \mathrm{~Hz}), 68.7,55.4(2 \mathrm{C}), 55.3,39.9$ (t, $J=2.5 \mathrm{~Hz}), 39.4,34.2$, 29.1, $21.7 \mathrm{ppm}$.

${ }^{19}$ F NMR (376 MHz, Chloroform- $d$ ) $\delta=-88.77$ (d, $\left.J=39.0 \mathrm{~Hz}, 1 \mathrm{~F}\right),-89.65$ (d, $J=39.3$ $\mathrm{Hz}, 1 \mathrm{~F}) \mathrm{ppm}$.

HRMS (ESI) m/z: [M+Na] ${ }^{+}$Calcd for $\mathrm{C}_{23} \mathrm{H}_{28} \mathrm{~F}_{2} \mathrm{O}_{4} \mathrm{Na}$ 429.1848; Found 429.1857

5,5-Difluoro-2-(4-methoxyphenethyl)-4-(2-methoxyphenyl)-2-methylpent-4-en-1-ol (3ca)<smiles>COc1ccc(CCC(C)(C)CC(=C(F)F)c2ccccc2OC)cc1</smiles>

The title compound 3ca was isolated as a colorless oil $(81 \mathrm{mg}, 54 \%)$ through flash chromatography on silica gel eluting with petroleum ether/ethyl acetate $(3: 1)$.

${ }^{1}$ H NMR (400 MHz, Chloroform-d) $\delta=7.34-7.24$ (m, 1H), 7.25-7.18 (m, 1H), 7.00$6.91(\mathrm{~m}, 1 \mathrm{H}), 6.92-6.85(\mathrm{~m}, 3 \mathrm{H}), 6.76(\mathrm{~d}, J=8.6 \mathrm{~Hz}, 2 \mathrm{H}), 3.81(\mathrm{~s}, 3 \mathrm{H}), 3.75(\mathrm{~s}, 3 \mathrm{H})$, 
$3.26(\mathrm{~s}, 2 \mathrm{H}), 2.50-2.43(\mathrm{~m}, 2 \mathrm{H}), 2.41-2.34(\mathrm{~m}, 2 \mathrm{H}), 1.49-1.41(\mathrm{~m}, 2 \mathrm{H}), 0.86(\mathrm{~s}, 3 \mathrm{H})$ ppm.

${ }^{13}$ C NMR (101 MHz, Chloroform- $d$ ) $\delta=157.6,156.8,153.8(\mathrm{dd}, J=289.8,289.9 \mathrm{~Hz})$, 135.0, 130.5 (t, $J=2.3 \mathrm{~Hz}), 129.2,129.1$ (2C), 123.8 (dd, $J=4.9,1.9 \mathrm{~Hz}), 120.7,113.7$ (2C), 111.3, 86.1 (dd, $J=23.1,16.4 \mathrm{~Hz}), 69.1,55.5,55.2,39.9$ (t, $J=2.4 \mathrm{~Hz}), 39.2,34.3$, 29.1, $21.9 \mathrm{ppm}$.

${ }^{19}$ F NMR (376 MHz, Chloroform-d) $\delta=-87.20$ (d, $\left.J=38.4 \mathrm{~Hz}, 1 \mathrm{~F}\right),-90.74$ (d, $J=38.2$ $\mathrm{Hz}, 1 \mathrm{~F}) \mathrm{ppm}$.

HRMS (ESI) m/z: [M+Na] ${ }^{+}$Calcd for $\mathrm{C}_{22} \mathrm{H}_{26} \mathrm{~F}_{2} \mathrm{O}_{3} \mathrm{Na}$ 399.1742; Found 399.1743

4-(3-(Benzyloxy)phenyl)-5,5-difluoro-2-(4-methoxyphenethyl)-2-methylpent-4-en-1-ol (3da)<smiles>COc1ccc(CCC(C)(CO)CC(=C(F)F)c2cccc(OCc3ccccc3)c2)cc1</smiles>

The title compound 3da was isolated as a colorless oil (134 mg, 74\%) through flash chromatography on silica gel eluting with petroleum ether/ethyl acetate (3:1).

${ }^{1}$ H NMR (400 MHz, Chloroform- $d$ ) $\delta=7.44-7.21$ (m, 6H), 6.98-6.86 (m, 5H), 6.77 (d, $J=8.6 \mathrm{~Hz}, 2 \mathrm{H}), 5.03(\mathrm{~s}, 2 \mathrm{H}), 3.73(\mathrm{~s}, 3 \mathrm{H}), 3.18(\mathrm{~s}, 2 \mathrm{H}), 2.54-2.35(\mathrm{~m}, 4 \mathrm{H}), 1.49-1.41$ (m, 2H), $0.81(\mathrm{~s}, 3 \mathrm{H}) \mathrm{ppm}$.

${ }^{13}$ C NMR (101 MHz, Chloroform- $d$ ) $\delta=158.9,157.7,154.5$ (t, $\left.J=288.9 \mathrm{~Hz}\right), 136.9$, 136.7, 134.9, 129.7, 129.2 (2C), 128.7 (2C), 128.1, 127.6 (2C), 121.1 (t, $J=2.8 \mathrm{~Hz}$ ), $115.5(\mathrm{t}, J=3.0 \mathrm{~Hz}), 113.84,113.81(2 \mathrm{C}), 90.2(\mathrm{dd}, J=21.6,13.6 \mathrm{~Hz}), 70.1,68.7,55.3$, $40.0(\mathrm{~d}, J=2.6 \mathrm{~Hz}), 39.3,34.3,29.1,21.8 \mathrm{ppm}$.

${ }^{19}$ F NMR (376 MHz, Chloroform- $d$ ) $\delta=-88.91$ (d, $\left.J=39.6 \mathrm{~Hz}, 1 \mathrm{~F}\right),-90.31$ (d, $J=39.5$ $\mathrm{Hz}, 1 \mathrm{~F}) \mathrm{ppm}$.

HRMS (ESI) m/z: [M+Na] $]^{+}$Calcd for $\mathrm{C}_{28} \mathrm{H}_{30} \mathrm{~F}_{2} \mathrm{O}_{3} \mathrm{Na}$ 475.2055; Found 475.2062

4-(Benzo[d][1,3]dioxol-5-yl)-5,5-difluoro-2-(4-methoxyphenethyl)-2-methylpent-4en-1-ol (3ea)<smiles>COc1ccc(CCC(C)(C)CC(=C(F)F)c2ccc3c(c2)OCO3)cc1</smiles>

The title compound 3ea was isolated as a colorless oil (110 mg, 71\%) through flash chromatography on silica gel eluting with petroleum ether/ethyl acetate (3:1).

${ }^{1}$ H NMR (400 MHz, Chloroform-d) $\delta=6.98(\mathrm{~d}, J=8.5 \mathrm{~Hz}, 2 \mathrm{H}), 6.81-6.77(\mathrm{~m}, 5 \mathrm{H})$, $5.94(\mathrm{~s}, 2 \mathrm{H}), 3.76(\mathrm{~s}, 3 \mathrm{H}), 3.28-3.18(\mathrm{~m}, 2 \mathrm{H}), 2.48-2.34(\mathrm{~m}, 4 \mathrm{H}), 1.55-1.45(\mathrm{~m}, 2 \mathrm{H})$, 0.84 (s, 3H) ppm. 
${ }^{13}$ C NMR (101 MHz, Chloroform- $\left.d\right) \delta=157.7,154.4(\mathrm{dd}, J=289.6,287.5 \mathrm{~Hz}), 147.8$, 146.9, 134.8, 129.1 (2C), 128.7 (dd, $J=4.8,2.3 \mathrm{~Hz}), 121.9$ (t, $J=2.7 \mathrm{~Hz}), 113.8$ (2C), $109.0(\mathrm{t}, J=2.9 \mathrm{~Hz}), 108.5,101.3,89.9(\mathrm{dd}, J=22.0,13.9 \mathrm{~Hz}), 68.7,55.3,39.9(\mathrm{t}, J=$ $2.4 \mathrm{~Hz}), 39.3,34.5,29.1,21.7 \mathrm{ppm}$.

${ }^{19}$ F NMR (376 MHz, Chloroform- $d$ ) $\delta=-89.63(\mathrm{~d}, J=41.6 \mathrm{~Hz}, 1 \mathrm{~F}),-91.07$ (d, $J=41.7$ $\mathrm{Hz}, 1 \mathrm{~F}) \mathrm{ppm}$.

HRMS (ESI) m/z: [M+Na] ${ }^{+}$Calcd for $\mathrm{C}_{22} \mathrm{H}_{24} \mathrm{~F}_{2} \mathrm{O}_{4} \mathrm{Na}$ 413.1535; Found 413.1542

4-(4-(tert-Butyl)phenyl)-5,5-difluoro-2-(4-methoxyphenethyl)-2-methylpent-4-en-1-ol (3fa)<smiles>COc1ccc(CCC(C)(CO)CC(=C(F)F)c2ccc(C(C)(C)C)cc2)cc1</smiles>

The title compound 3fa was isolated as a colorless oil (129 mg, 80\%) through flash chromatography on silica gel eluting with petroleum ether/ethyl acetate (3:1).

${ }^{1}$ H NMR (400 MHz, Chloroform-d) $\delta=7.37$ (d, $\left.J=8.5 \mathrm{~Hz}, 2 \mathrm{H}\right), 7.27(\mathrm{~d}, J=8.6 \mathrm{~Hz}$, $2 \mathrm{H}), 6.84(\mathrm{~d}, J=8.6 \mathrm{~Hz}, 2 \mathrm{H}), 6.74(\mathrm{~d}, J=8.6 \mathrm{~Hz}, 2 \mathrm{H}), 3.73(\mathrm{~s}, 3 \mathrm{H}), 3.29-3.20(\mathrm{~m}, 2 \mathrm{H})$, 2.50-2.33 (m, 4H), 1.47-1.37 (m, 2H), $1.32(\mathrm{~s}, 9 \mathrm{H}), 0.87$ (s, 3H) ppm.

${ }^{13}$ C NMR (101 MHz, Chloroform-d) $\delta=157.6,154.4(\mathrm{dd}, J=290.1,287.3 \mathrm{~Hz}), 150.5$, 134.9, 132.0 (dd, $J=4.7,2.4 \mathrm{~Hz}), 129.1$ (2C), 128.1 (t, $J=2.7 \mathrm{~Hz}, 2 \mathrm{C}), 125.6$ (2C), 113.7 (2C), 89.9 (dd, $J=21.3,13.9 \mathrm{~Hz}), 68.9,55.2,39.9$ (t, $J=2.5 \mathrm{~Hz}), 39.3,34.6,34.0$, 31.4 (3C), 29.1, 21.9 ppm.

${ }^{19}$ F NMR (376 MHz, Chloroform- $d$ ) $\delta=-89.46(\mathrm{~d}, J=41.2 \mathrm{~Hz}, 1 \mathrm{~F}),-91.41$ (d, $J=41.6$ $\mathrm{Hz}, 1 \mathrm{~F}) \mathrm{ppm}$.

HRMS (ESI) m/z: [M+Na] $]^{+}$Calcd for $\mathrm{C}_{25} \mathrm{H}_{32} \mathrm{~F}_{2} \mathrm{O}_{2} \mathrm{Na}$ 425.2263; Found 425.2266

5,5-Difluoro-4-(2-fluoro-4-methoxyphenyl)-2-(4-methoxyphenethyl)-2-methylpent-4en-1-ol (3ga)<smiles>COc1ccc(CCC(C)(CO)CC(=C(F)F)c2ccc(OC)cc2F)cc1</smiles>

The title compound 3ga was isolated as a colorless oil (124 mg, 79\%) through flash chromatography on silica gel eluting with petroleum ether/ethyl acetate $(3: 1)$.

${ }^{1}$ H NMR (400 MHz, Chloroform-d) $\delta=7.17(\mathrm{t}, J=8.5 \mathrm{~Hz}, 1 \mathrm{H}), 6.93(\mathrm{~d}, J=8.6 \mathrm{~Hz}$, $2 \mathrm{H}), 6.77(\mathrm{~d}, J=8.6 \mathrm{~Hz}, 2 \mathrm{H}), 6.70(\mathrm{dd}, J=8.6,2.6 \mathrm{~Hz}, 1 \mathrm{H}), 6.63(\mathrm{dd}, J=11.9,2.6 \mathrm{~Hz}$, $1 \mathrm{H}), 3.78(\mathrm{~s}, 3 \mathrm{H}), 3.76(\mathrm{~s}, 3 \mathrm{H}), 3.31-3.14(\mathrm{~m}, 2 \mathrm{H}), 2.49-2.33(\mathrm{~m}, 4 \mathrm{H}), 1.54-1.40(\mathrm{~m}$, $2 \mathrm{H}), 0.85$ (s, 3H) ppm. 
${ }^{13}$ C NMR (101 MHz, Chloroform- $d$ ) $\delta=160.6(\mathrm{~d}, J=10.9 \mathrm{~Hz}), 160.3(\mathrm{~d}, J=247.6 \mathrm{~Hz})$, 157.7, 154.1 (t, $J=288.9 \mathrm{~Hz}), 134.8,130.9$ (t, $J=3.8 \mathrm{~Hz}), 129.1$ (2C), 114.6 (ddd, $J=$ 15.6, 5.0, $2.1 \mathrm{~Hz}), 113.7(2 \mathrm{C}), 110.3$ (d, $J=3.0 \mathrm{~Hz}), 101.9$ (d, $J=26.2 \mathrm{~Hz}), 83.9$ (dd, $J=$ 24.6, 16.4 Hz), 68.8, 55.6, 55.3, 39.8 (t, $J=2.5 \mathrm{~Hz}), 39.1,34.1,29.0,21.6 \mathrm{ppm}$.

${ }^{19}$ F NMR (376 MHz, Chloroform-d) $\delta=-87.19(\mathrm{dd}, J=37.0,15.0 \mathrm{~Hz}, 1 \mathrm{~F}),-89.43(\mathrm{~d}$, $J=37.3 \mathrm{~Hz}, 1 \mathrm{~F}),-110.43--111.54(\mathrm{~m}, 1 \mathrm{~F}) \mathrm{ppm}$.

HRMS (ESI) m/z: [M+Na] $]^{+}$Calcd for $\mathrm{C}_{22} \mathrm{H}_{25} \mathrm{~F}_{3} \mathrm{O}_{3} \mathrm{Na}$ 417.1648; Found 417.1653

4-(3-Chloro-4-methoxyphenyl)-5,5-difluoro-2-(4-methoxyphenethyl)-2-methylpent-4en-1-ol (3ha)<smiles>COc1ccc(CCC(C)(C)CC(=C(F)F)c2ccc(OC)c(Cl)c2)cc1</smiles>

The title compound 3ha was isolated as a colorless oil (133 mg, 81\%) through flash chromatography on silica gel eluting with petroleum ether/ethyl acetate $(3: 1)$.

${ }^{1}$ H NMR (400 MHz, Chloroform-d) $\delta=7.56-7.50(\mathrm{~m}, 1 \mathrm{H}), 7.24(\mathrm{~d}, J=8.4 \mathrm{~Hz}, 1 \mathrm{H})$, $6.96(\mathrm{~d}, J=8.6 \mathrm{~Hz}, 2 \mathrm{H}), 6.85(\mathrm{~d}, J=8.5 \mathrm{~Hz}, 1 \mathrm{H}), 6.78(\mathrm{~d}, J=8.6 \mathrm{~Hz}, 2 \mathrm{H}), 3.87(\mathrm{~s}, 3 \mathrm{H})$, $3.76(\mathrm{~s}, 3 \mathrm{H}), 3.28-3.01(\mathrm{~m}, 2 \mathrm{H}), 2.51-2.36(\mathrm{~m}, 4 \mathrm{H}), 1.55-1.42(\mathrm{~m}, 2 \mathrm{H}), 0.83(\mathrm{~s}, 3 \mathrm{H})$ ppm.

${ }^{13}$ C NMR (101 MHz, Chloroform-d) $\delta=157.7,155.1,154.5$ (dd, $\left.J=290.3,288.1 \mathrm{~Hz}\right)$, 134.7, 133.2 (t, $J=2.9 \mathrm{~Hz}), 129.1$ (2C) 128.7 (dd, $J=4.9,2.5 \mathrm{~Hz}), 128.6$ (t, $J=2.8 \mathrm{~Hz}$ ), $113.8(2 \mathrm{C}), 111.8,111.7,88.9$ (dd, $J=22.4,13.9 \mathrm{~Hz}), 68.6,56.3,55.3,39.9$ (t, $J=2.4$ $\mathrm{Hz}), 39.2,34.2,29.0,21.8 \mathrm{ppm}$.

${ }^{19}$ F NMR (376 MHz, Chloroform- $d$ ) $\delta=-89.07$ (d, $\left.J=42.3 \mathrm{~Hz}, 1 \mathrm{~F}\right),-91.43(\mathrm{~d}, J=42.4$ $\mathrm{Hz}, 1 \mathrm{~F}) \mathrm{ppm}$.

HRMS (ESI) m/z: [M+H] $]^{+}$Calcd for $\mathrm{C}_{22} \mathrm{H}_{25} \mathrm{ClF}_{2} \mathrm{O}_{3} 411.1533$; Found 411.1547

4-(3-Bromo-4-methoxyphenyl)-5,5-difluoro-2-(4-methoxyphenethyl)-2-methylpent-4en-1-ol (3ia)<smiles>COc1ccc(CCC(C)(C)CC(=C(F)F)c2ccc(OC)c(Br)c2)cc1</smiles>

The title compound 3ia was isolated as a colorless oil (129 mg, 71\%) through flash chromatography on silica gel eluting with petroleum ether/ethyl acetate (3:1).

${ }^{1}$ H NMR (400 MHz, Chloroform-d) $\delta=7.33(\mathrm{~d}, J=8.7 \mathrm{~Hz}, 1 \mathrm{H}), 6.94(\mathrm{~d}, J=8.6 \mathrm{~Hz}$, $2 \mathrm{H}), 6.91-6.84(\mathrm{~m}, 2 \mathrm{H}), 6.79(\mathrm{~d}, J=8.6 \mathrm{~Hz}, 2 \mathrm{H}), 3.87(\mathrm{~s}, 3 \mathrm{H}), 3.76(\mathrm{~s}, 3 \mathrm{H}), 3.29-3.13$ $(\mathrm{m}, 2 \mathrm{H}), 2.50-2.38(\mathrm{~m}, 4 \mathrm{H}), 1.56-1.45(\mathrm{~m}, 2 \mathrm{H}), 0.84(\mathrm{~s}, 3 \mathrm{H}) \mathrm{ppm}$. 
${ }^{13}$ C NMR (101 MHz, Chloroform-d) $\delta=157.7,154.9,154.4(\mathrm{dd}, J=291.0,288.4 \mathrm{~Hz})$, $135.1(\mathrm{dd}, J=5.0,2.6 \mathrm{~Hz}), 134.7,130.2,129.1(2 \mathrm{C}), 121.6,121.4(\mathrm{t}, J=2.8 \mathrm{~Hz}), 113.8$ (2C), 112.5 (t, $J=2.9 \mathrm{~Hz}$ ), 89.9 (dd, $J=22.1,13.5 \mathrm{~Hz}$ ), 68.6, 56.2, 55.3, 39.9 (t, $J=2.4$ $\mathrm{Hz}), 39.3,34.2,29.1,21.7 \mathrm{ppm}$.

${ }^{19}$ F NMR (376 MHz, Chloroform- $d$ ) $\delta=-88.29(\mathrm{~d}, J=38.3 \mathrm{~Hz}, 1 \mathrm{~F}),-89.69$ (d, $J=38.7$ $\mathrm{Hz}, 1 \mathrm{~F}) \mathrm{ppm}$.

HRMS (ESI) m/z: [M+Na] ${ }^{+}$Calcd for $\mathrm{C}_{22} \mathrm{H}_{25} \mathrm{BrF}_{2} \mathrm{O}_{3} \mathrm{Na}_{4} 47.0847$; Found 477.0849

4-(4-Chlorophenyl)-5,5-difluoro-2-(4-methoxyphenethyl)-2-methylpent-4-en-1-ol (3ja)<smiles>COc1ccc(CCC(C)(C)CC(=C(F)F)c2ccc(Cl)cc2)cc1</smiles>

The title compound 3ja was isolated as a colorless oil (128 mg, 84\%) through flash chromatography on silica gel eluting with petroleum ether/ethyl acetate (3:1).

${ }^{1} \mathbf{H}$ NMR (400 MHz, Chloroform-d) $\delta=7.32(\mathrm{~d}, J=8.6 \mathrm{~Hz}, 2 \mathrm{H}), 7.26(\mathrm{~d}, J=8.7 \mathrm{~Hz}$, $2 \mathrm{H}), 6.92(\mathrm{~d}, J=8.6 \mathrm{~Hz}, 2 \mathrm{H}), 6.78(\mathrm{~d}, J=8.6 \mathrm{~Hz}, 2 \mathrm{H}), 3.75(\mathrm{~s}, 3 \mathrm{H}), 3.27-3.14(\mathrm{~m}, 2 \mathrm{H})$, 2.50-2.36 (m, 4H), 1.52-1.39 (m, 2H), $0.81(\mathrm{~s}, 3 \mathrm{H}) \mathrm{ppm}$.

${ }^{13}$ C NMR (101 MHz, Chloroform-d) $\delta=157.7,154.4(\mathrm{dd}, J=290.9,288.4 \mathrm{~Hz}), 134.6$, 133.7 (dd, $J=4.9,2.6 \mathrm{~Hz}$ ), 133.2, 129.8 (t, $J=2.8 \mathrm{~Hz}, 2 \mathrm{C}$ ), 129.1 (2C), 128.8 (2C), 113.8 (2C), 89.5 (dd, $J=22.3,13.6 \mathrm{~Hz}), 68.6,55.3,39.9$ (t, $J=2.4 \mathrm{~Hz}), 39.2,34.1,29.0$, $21.7 \mathrm{ppm}$.

${ }^{19}$ F NMR (376 MHz, Chloroform- $d$ ) $\delta=-88.40(\mathrm{~d}, J=38.8 \mathrm{~Hz}, 1 \mathrm{~F}),-90.36$ (d, $J=38.9$ $\mathrm{Hz}, 1 \mathrm{~F}) \mathrm{ppm}$.

HRMS (ESI) m/z: [M+Na] ${ }^{+}$Calcd for $\mathrm{C}_{21} \mathrm{H}_{23} \mathrm{~F}_{2} \mathrm{O}_{2} \mathrm{ClNa} 403.1247$; Found 403.1235

5,5-Difluoro-2-(4-methoxyphenethyl)-2-methyl-4-(4-(pyridin-2-yl)phenyl)pent-4-en-1ol (3ka)<smiles>COc1ccc(CCC(C)(C)CC(=C(F)F)c2ccc(-c3ccccn3)cc2)cc1</smiles>

The title compound 3ka was isolated as a colorless oil (102 mg, 60\%) through flash chromatography on silica gel eluting with petroleum ether/ethyl acetate (2:1).

${ }^{1}$ H NMR (400 MHz, Chloroform-d) $\delta=8.78-8.48(\mathrm{~m}, 1 \mathrm{H}), 7.97(\mathrm{~d}, J=8.4 \mathrm{~Hz}, 2 \mathrm{H})$, 7.75-7.65 (m, 2H), $7.45(\mathrm{dd}, J=8.5,1.6 \mathrm{~Hz}, 2 \mathrm{H}), 7.30-7.13(\mathrm{~m}, 1 \mathrm{H}), 6.93(\mathrm{~d}, J=8.6 \mathrm{~Hz}$, $2 \mathrm{H}), 6.74(\mathrm{~d}, J=8.7 \mathrm{~Hz}, 2 \mathrm{H}), 3.72(\mathrm{~s}, 3 \mathrm{H}), 3.29-3.10(\mathrm{~m}, 2 \mathrm{H}), 2.60-2.37(\mathrm{~m}, 4 \mathrm{H}), 1.57-$ $1.43(\mathrm{~m}, 2 \mathrm{H}), 0.83(\mathrm{~s}, 3 \mathrm{H}) \mathrm{ppm}$. 
${ }^{13}$ C NMR (101 MHz, Chloroform-d) $\delta=157.6,156.8,154.5$ (dd, $\left.J=290.9,288.9 \mathrm{~Hz}\right)$, 149.7, 138.4, 136.9, 135.9 (dd, $J=4.7,2.7 \mathrm{~Hz}$ ), 134.8, 129.1 (2C), 128.9 (t, $J=2.7 \mathrm{~Hz}$, 2C), 127.1 (2C), 122.3, 120.6, 113.7 (2C), $90.0(\mathrm{dd}, J=21.7,13.5 \mathrm{~Hz}), 68.5,55.2,39.9$ (t, J=2.4 Hz), 39.3, 34.0, 29.1, $21.8 \mathrm{ppm}$.

${ }^{19}$ F NMR (376 MHz, Chloroform- $d$ ) $\delta=-92.10(\mathrm{~d}, J=45.9 \mathrm{~Hz}, 1 \mathrm{~F}),-92.32(\mathrm{~d}, J=46.0$ $\mathrm{Hz}, 1 \mathrm{~F}) \mathrm{ppm}$.

HRMS (ESI) m/z: [M+H] $]^{+}$Calcd for $\mathrm{C}_{26} \mathrm{H}_{28} \mathrm{~F}_{2} \mathrm{NO}_{2}$ 424.2083; Found 424.2087

5,5-Difluoro-4-(3-(hydroxymethyl)phenyl)-2-(4-methoxyphenethyl)-2-methylpent-4en-1-ol (3la)<smiles>COc1ccc(CCC(C)(C)CC(=C(F)F)c2cccc(CO)c2)cc1</smiles>

The title compound 3la was isolated as a colorless oil (99 mg, 66\%) through flash chromatography on silica gel eluting with petroleum ether/ethyl acetate (1:1).

${ }^{1}$ H NMR (400 MHz, Chloroform- $d$ ) $\delta=7.39-7.29$ (m, 2H), 7.30-7.20 (m, 2H), 6.93 (d, $J=8.5 \mathrm{~Hz}, 2 \mathrm{H}), 6.77(\mathrm{~d}, J=8.6 \mathrm{~Hz}, 2 \mathrm{H}), 4.62(\mathrm{~s}, 2 \mathrm{H}), 3.76(\mathrm{~s}, 3 \mathrm{H}), 3.18-3.07(\mathrm{~m}, 2 \mathrm{H})$, 2.51-2.40 (m, 4H), 1.51-1.43 (m, 2H), $0.84(\mathrm{~s}, 3 \mathrm{H}) \mathrm{ppm}$.

${ }^{13}$ C NMR (101 MHz, Chloroform-d) $\delta=157.7,154.5$ (dd, $\left.J=290.5,288.0 \mathrm{~Hz}\right), 141.3$, 135.4 (dd, $J=4.7,2.5 \mathrm{~Hz}), 134.8,129.1$ (2C), 128.7, 127.7 (t, $J=2.7 \mathrm{~Hz}), 126.9$ (t, $J=$ $2.7 \mathrm{~Hz}), 126.1,113.8(2 \mathrm{C}), 90.1(\mathrm{dd}, J=21.4,13.7 \mathrm{~Hz}), 68.4,64.9,55.3,39.9$ (t, $J=2.4$ $\mathrm{Hz}), 39.3,33.9,28.9,21.6 \mathrm{ppm}$.

${ }^{19}$ F NMR (376 MHz, Chloroform- $d$ ) $\delta=-89.05$ (d, $\left.J=40.2 \mathrm{~Hz}, 1 \mathrm{~F}\right),-90.88$ (d, $J=39.9$ $\mathrm{Hz}, 1 \mathrm{~F}) \mathrm{ppm}$.

HRMS (ESI) m/z: [M+Na] $]^{+}$Calcd for $\mathrm{C}_{22} \mathrm{H}_{26} \mathrm{~F}_{2} \mathrm{O}_{3} \mathrm{Na} 399.1742$; Found 399.1746

1-(3-(1,1-Difluoro-4-(hydroxymethyl)-6-(4-methoxyphenyl)-4-methylhex-1-en-2yl)phenyl)ethan-1-one (3ma)<smiles>COc1ccc(CCC(C)(C)CC(=C(F)F)c2cccc(C(C)=O)c2)cc1</smiles>

The title compound 3ma was isolated as a colorless oil (96 mg, 62\%) through flash chromatography on silica gel eluting with petroleum ether/ethyl acetate (3:1).

${ }^{1}$ H NMR (400 MHz, Chloroform- $d$ ) $\delta=7.95(\mathrm{~s}, 1 \mathrm{H}), 7.89-7.81(\mathrm{~m}, 1 \mathrm{H}), 7.60-7.53(\mathrm{~m}$, $1 \mathrm{H}), 7.46(\mathrm{t}, J=7.7 \mathrm{~Hz}, 1 \mathrm{H}), 6.93(\mathrm{~d}, J=8.5 \mathrm{~Hz}, 2 \mathrm{H}), 6.77$ (d, $J=8.6 \mathrm{~Hz}, 2 \mathrm{H}), 3.77$ (s, $3 \mathrm{H}), 3.32-3.05(\mathrm{~m}, 2 \mathrm{H}), 2.60(\mathrm{~s}, 3 \mathrm{H}), 2.56-2.49(\mathrm{~m}, 2 \mathrm{H}), 2.43(\mathrm{t}, J=8.7 \mathrm{~Hz}, 2 \mathrm{H}), 1.56-$ $1.42(\mathrm{~m}, 2 \mathrm{H}), 0.83(\mathrm{~s}, 3 \mathrm{H}) \mathrm{ppm}$. 
${ }^{13}$ C NMR (101 MHz, Chloroform-d) $\delta=197.8,157.7,154.6(\mathrm{dd}, J=291.2,288.6 \mathrm{~Hz})$, $137.3,135.9$ (dd, $J=5.1,2.6 \mathrm{~Hz}), 134.6,133.1$ (t, $J=2.8 \mathrm{~Hz}), 129.0(2 \mathrm{C}), 128.8,128.1$ (t, $J=2.8 \mathrm{~Hz}), 127.4,113.8(2 \mathrm{C}), 89.8$ (dd, $J=22.1,13.5 \mathrm{~Hz}), 68.6,55.3,39.9$ (t, $J=2.4$ $\mathrm{Hz}), 39.2,34.1,28.9,26.7,21.8 \mathrm{ppm}$.

${ }^{19}$ F NMR (376 MHz, Chloroform- $d$ ) $\delta=-88.12(\mathrm{~d}, J=38.8 \mathrm{~Hz}, 1 \mathrm{~F}),-90.23$ (d, $J=38.1$ $\mathrm{Hz}, 1 \mathrm{~F}) \mathrm{ppm}$.

HRMS (ESI) m/z: [M+Na] $]^{+}$Calcd for $\mathrm{C}_{23} \mathrm{H}_{26} \mathrm{~F}_{2} \mathrm{O}_{3} \mathrm{Na} 411.1742$; Found 411.1749

5,5-Difluoro-2-(4-methoxyphenethyl)-4-(6-methoxypyridin-3-yl)-2-methylpent-4-en-1ol (3na)<smiles>COc1ccc(CCC(C)(CO)CC(=C(F)F)c2ccc(OC)nc2)cc1</smiles>

The title compound 3na was isolated as a colorless oil (116 mg, 77\%) through flash chromatography on silica gel eluting with petroleum ether/ethyl acetate (3:1).

${ }^{1}$ H NMR (400 MHz, Chloroform- $d$ ) $\delta=8.20-8.08(\mathrm{~m}, 1 \mathrm{H}), 7.57-7.49$ (m, 1H), $6.96(\mathrm{~d}$, $J=8.6 \mathrm{~Hz}, 2 \mathrm{H}), 6.78(\mathrm{~d}, J=8.7 \mathrm{~Hz}, 2 \mathrm{H}), 6.77-6.70(\mathrm{~m}, 1 \mathrm{H}), 3.93(\mathrm{~s}, 3 \mathrm{H}), 3.76(\mathrm{~s}, 3 \mathrm{H})$, 3.29-3.20 (m, 2H), 2.51-2.36 (m, 4H), 1.57-1.40 (m, 2H), $0.83(\mathrm{~s}, 3 \mathrm{H}) \mathrm{ppm}$.

${ }^{13}$ C NMR (101 MHz, Chloroform-d) $\delta=163.2,157.7,154.5$ (dd, $J=290.1,288.4 \mathrm{~Hz}$ ), $146.4(\mathrm{t}, J=3.1 \mathrm{~Hz}), 138.6(\mathrm{t}, J=2.7 \mathrm{~Hz}), 134.6,129.1(2 \mathrm{C}), 123.9$ (dd, $J=4.8,2.8 \mathrm{~Hz})$, 113.8 (2C), 110.7, 87.1 (dd, $J=22.9,14.1 \mathrm{~Hz}), 68.5,55.2,53.5,39.8$ (t, $J=2.4 \mathrm{~Hz}$ ), 39.3, 33.9, 29.0, $21.8 \mathrm{ppm}$.

${ }^{19}$ F NMR (376 MHz, Chloroform- $d$ ) $\delta=-88.52(\mathrm{~d}, J=40.1 \mathrm{~Hz}, 1 \mathrm{~F}),-90.90$ (d, $J=40.3$ $\mathrm{Hz}, 1 \mathrm{~F}) \mathrm{ppm}$.

HRMS (ESI) m/z: $[\mathrm{M}+\mathrm{H}]^{+}$Calcd for $\mathrm{C}_{21} \mathrm{H}_{26} \mathrm{~F}_{2} \mathrm{NO}_{3}$ 378.1875; Found 378.1878

tert-Butyl 3-(1,1-difluoro-4-(hydroxymethyl)-6-(4-methoxyphenyl)-4-methylhex-1-en2-yl)-1H-indole-1-carboxylate (3oa)<smiles>COc1ccc(CCC(C)(C)CC(=C(F)F)c2cn(C(C)(C)C)c3ccccc23)cc1</smiles>

The title compound 3oa was isolated as a colorless oil (136 mg, 70\%) through flash chromatography on silica gel eluting with petroleum ether/ethyl acetate $(2: 1)$.

${ }^{1} \mathbf{H}$ NMR (400 MHz, Chloroform-d) $\delta=8.16(\mathrm{~d}, J=8.3 \mathrm{~Hz}, 1 \mathrm{H}), 7.63-7.48(\mathrm{~m}, 2 \mathrm{H})$, 7.39-7.22 (m, 2H), $6.86(\mathrm{~d}, J=8.6 \mathrm{~Hz}, 2 \mathrm{H}), 6.74(\mathrm{~d}, J=8.6 \mathrm{~Hz}, 2 \mathrm{H}), 3.75(\mathrm{~s}, 3 \mathrm{H}), 3.27$ (s, 2H), 2.56-2.49 (m, 2H), 2.45-2.37 (m, 2H), $1.66(\mathrm{~s}, 9 \mathrm{H}), 1.56-1.41(\mathrm{~m}, 2 \mathrm{H}), 0.88$ (s, $3 \mathrm{H}) \mathrm{ppm}$. 
${ }^{13}$ C NMR (101 MHz, Chloroform- $\left.d\right) \delta=157.6,154.4(\mathrm{dd}, J=290.9,288.6 \mathrm{~Hz}), 149.5$, 135.3, 134.7, 129.0 (2C), 128.7, 124.8, 124.3 (d, $J=4.4$ Hz), 123.0, 120.0 (d, $J=3.2$ $\mathrm{Hz}), 115.5,115.3$ (dd, $J=5.3,1.7 \mathrm{~Hz}), 113.8$ (2C), 84.2, 82.2 (dd, $J=25.1,15.6 \mathrm{~Hz}$ ), 68.8, 55.3, 39.9 (t, $J=2.4 \mathrm{~Hz}), 39.1,34.9,29.1,28.2(3 \mathrm{C}), 21.7 \mathrm{ppm}$.

${ }^{19}$ F NMR (376 MHz, Chloroform- $d$ ) $\delta=-85.36(\mathrm{~d}, J=36.8 \mathrm{~Hz}, 1 \mathrm{~F}),-88.27$ (d, $J=36.9$ $\mathrm{Hz}, 1 \mathrm{~F}) \mathrm{ppm}$.

HRMS (ESI) m/z: [M+Na] ${ }^{+}$Calcd for $\mathrm{C}_{28} \mathrm{H}_{33} \mathrm{~F}_{2} \mathrm{NO}_{4} \mathrm{Na}$ 508.2270; Found 508.2273

4-(Benzo[b]thiophen-3-yl)-5,5-difluoro-2-(4-methoxyphenethyl)-2-methylpent-4-en-1ol (3pa)<smiles>COc1ccc(CCC(C)(C)CC(=C(F)F)c2csc3ccccc23)cc1</smiles>

The title compound 3pa was isolated as a colorless oil (125 mg, 78\%) through flash chromatography on silica gel eluting with petroleum ether/ethyl acetate (3:1).

${ }^{1}$ H NMR (400 MHz, Chloroform-d) $\delta=7.86(\mathrm{~d}, J=7.2 \mathrm{~Hz}, 1 \mathrm{H}), 7.71(\mathrm{~d}, J=7.7 \mathrm{~Hz}$, $1 \mathrm{H}), 7.45-7.32(\mathrm{~m}, 3 \mathrm{H}), 6.84(\mathrm{~d}, J=8.3 \mathrm{~Hz}, 2 \mathrm{H}), 6.73(\mathrm{~d}, J=8.4 \mathrm{~Hz}, 2 \mathrm{H}), 3.74(\mathrm{~s}, 3 \mathrm{H})$, 3.27-3.11 (m, 2H), 2.56 (t, J=2.4 Hz, 2H), 2.40-2.32 (m, 2H), 1.53-1.41 (m, 2H), 0.86 (s, 3H) ppm.

${ }^{13}$ C NMR (101 MHz, Chloroform- $d$ ) $\delta=157.7,154.3(\mathrm{t}, J=291.9 \mathrm{~Hz}), 140.1,137.2$ (d, $J=1.8 \mathrm{~Hz}$ ), 134.7, 130.6 (dd, $J=5.0,1.8 \mathrm{~Hz}$ ), 129.1 (2C), 125.0 (d, $J=3.3 \mathrm{~Hz}$ ), 124.7, 124.6, 123.1, 122.8 (d, $J=2.9 \mathrm{~Hz}), 113.8(2 \mathrm{C}), 84.5$ (dd, $J=24.1,15.9 \mathrm{~Hz}), 68.8,55.3$, $39.9(\mathrm{t}, J=2.4 \mathrm{~Hz}), 39.2,35.4,29.1,21.7 \mathrm{ppm}$.

${ }^{19}$ F NMR (376 MHz, Chloroform- $d$ ) $\delta=-83.97$ (d, $\left.J=35.3 \mathrm{~Hz}, 1 \mathrm{~F}\right),-88.28$ (d, $J=35.4$ $\mathrm{Hz}, 1 \mathrm{~F}) \mathrm{ppm}$.

HRMS (ESI) m/z: $[\mathrm{M}+\mathrm{H}]^{+}$Calcd for $\mathrm{C}_{23} \mathrm{H}_{25} \mathrm{~F}_{2} \mathrm{O}_{2} \mathrm{~S} 403.1538$; Found 403.1542

(8R,9S,13S,14S)-3-(1,1-Difluoro-4-(hydroxymethyl)-6-(4-methoxyphenyl)-4methylhex-1-en-2-yl)-13-methyl-6,7,8,9,11,12,13,14,15,16-decahydro-17Hcyclopenta[a]phenanthren-17-one (3qa)<smiles>COc1ccc(CCC(C)(C)CC(=C(F)F)c2ccc3c(c2)CC[C@@H]2[C@@H]3CC[C@]3(C)C(=O)CC[C@H]23)cc1</smiles>

The title compound 3qa was isolated as a colorless oil (66 mg, 63\%) through flash chromatography on silica gel eluting with petroleum ether/ethyl acetate (3:1). 
${ }^{1} \mathbf{H}$ NMR (400 MHz, Chloroform-d) $\delta=7.27(\mathrm{~d}, J=8.1 \mathrm{~Hz}, 1 \mathrm{H}), 7.13(\mathrm{~d}, J=8.2 \mathrm{~Hz}$, 1H), $7.08(\mathrm{~s}, 1 \mathrm{H}), 6.88(\mathrm{~d}, J=8.1 \mathrm{~Hz}, 2 \mathrm{H}), 6.76(\mathrm{~d}, J=8.1 \mathrm{~Hz}, 2 \mathrm{H}), 3.75(\mathrm{~s}, 3 \mathrm{H}), 3.24$ (s, 2H), 2.94-2.84 (m, 2H), 2.56-2.36 (m, 6H), 2.35-2.23 (m, 1H), 2.19-1.93 (m, 4H), $1.68-1.40(\mathrm{~m}, 9 \mathrm{H}), 0.92(\mathrm{~s}, 3 \mathrm{H}), 0.88(\mathrm{~s}, 3 \mathrm{H}) \mathrm{ppm}$.

${ }^{13}$ C NMR (101 MHz, Chloroform- $\left.d\right) \delta=220.9,157.6,154.4(\mathrm{dd}, J=288.9,299.0 \mathrm{~Hz})$, 139.1, 136.8, 134.9, 132.5 (t, $J=3.4 \mathrm{~Hz}), 129.1(2 \mathrm{C}), 128.9$ (t, $J=2.9 \mathrm{~Hz}), 125.8$ (d, $J=$ $2.5 \mathrm{~Hz}), 125.6,113.7$ (2C), 89.8 (dd, $J=21.3,13.9 \mathrm{~Hz}), 68.9,55.2,50.5,48.0,44.3$, 39.9 (t, $J=2.9 \mathrm{~Hz}), 39.2$, 38.1, 35.9, 33.9, 31.6, 29.4, 29.1, 26.5, 25.7, 21.9, 21.6, 13.9 ppm.

${ }^{19}$ F NMR (376 MHz, Chloroform-d) $\delta=-89.37$ (d, $\left.J=41.1 \mathrm{~Hz}, 1 \mathrm{~F}\right),-91.24$ (d, $J=41.2$ $\mathrm{Hz}, 1 \mathrm{~F}) \mathrm{ppm}$.

HRMS (ESI) m/z: [M+Na] $]^{+}$Calcd for $\mathrm{C}_{33} \mathrm{H}_{40} \mathrm{~F}_{2} \mathrm{O}_{3} \mathrm{Na}$ 545.2838; Found 545.2838

5,5-Difluoro-4-(4-methoxyphenyl)-2,2-dimethylpent-4-en-1-ol (3ab)<smiles>COc1ccc(C(CC(C)(C)CO)=C(F)F)cc1</smiles>

The title compound 3ab was isolated as a colorless oil $(85 \mathrm{mg}, 83 \%)$ through flash chromatography on silica gel eluting with petroleum ether/ethyl acetate $(3: 1)$.

${ }^{1} \mathrm{H}$ NMR (400 MHz, Chloroform-d) $\delta=7.25(\mathrm{~d}, J=8.8 \mathrm{~Hz}, 2 \mathrm{H}), 6.88(\mathrm{~d}, J=8.8 \mathrm{~Hz}$, $2 \mathrm{H}), 3.80$ (s, 3H), 3.14 (s, 2H), 2.37 (t, J=2.4 Hz, 2H), 1.33 (brs, 1H), 0.80 (s, 6H) ppm. ${ }^{13}$ C NMR (101 MHz, Chloroform-d) $\delta=158.7,154.3$ (dd, $\left.J=289.4,286.9 \mathrm{~Hz}\right), 129.4$ (t, $J=2.9 \mathrm{~Hz}, 2 \mathrm{C}), 127.22$ (dd, $J=4.6,2.6 \mathrm{~Hz}), 113.9(2 \mathrm{C}), 89.8$ (dd, $J=21.7,13.7 \mathrm{~Hz})$, $71.1,55.2,37.3(\mathrm{t}, J=2.5 \mathrm{~Hz}), 36.0,24.3(2 \mathrm{C}) \mathrm{ppm}$.

${ }^{19}$ F NMR (376 MHz, Chloroform- $d$ ) $\delta=-90.51(\mathrm{~d}, J=43.0 \mathrm{~Hz}, 1 \mathrm{~F}),-92.57$ (d, $J=43.0$ $\mathrm{Hz}, 1 \mathrm{~F}) \mathrm{ppm}$.

HRMS (ESI) m/z: $[\mathrm{M}+\mathrm{H}]^{+}$Calcd for $\mathrm{C}_{14} \mathrm{H}_{19} \mathrm{~F}_{2} \mathrm{O}_{2} 257.1348$; Found 257.1355

2,2-Dibenzyl-5,5-difluoro-4-(4-methoxyphenyl)pent-4-en-1-ol (3ac)<smiles>COc1ccc(C(CC(C)(CBr)CBr)=C(F)F)cc1</smiles>

The title compound 3ac was isolated as a colorless oil (126 mg, 77\%) through flash chromatography on silica gel eluting with petroleum ether/ethyl acetate $(3: 1)$.

${ }^{1} \mathbf{H}$ NMR (400 MHz, Chloroform- $d$ ) $\delta=7.30-7.13(\mathrm{~m}, 10 \mathrm{H}), 7.12(\mathrm{~d}, J=8.8 \mathrm{~Hz}, 2 \mathrm{H})$, $6.85(\mathrm{~d}, J=8.7 \mathrm{~Hz}, 2 \mathrm{H}), 3.77(\mathrm{~s}, 3 \mathrm{H}), 3.19(\mathrm{~s}, 2 \mathrm{H}), 2.71(\mathrm{~s}, 4 \mathrm{H}), 2.45(\mathrm{t}, J=2.4 \mathrm{~Hz}, 2 \mathrm{H})$ ppm. 
${ }^{13}$ C NMR (101 MHz, Chloroform-d) $\delta=158.9,154.0(\mathrm{t}, J=289.9 \mathrm{~Hz}), 138.2(2 \mathrm{C})$, 130.7 (4C), 129.6 (t, $J=2.7 \mathrm{~Hz}, 2 \mathrm{C}$ ), 128.2 (4C), 126.9 (dd, $J=4.4,2.3 \mathrm{~Hz}$ ), 126.3 (2C), 114.1 (2C), 89.5 (dd, $J=20.9,14.8 \mathrm{~Hz}$ ), 66.6, 55.2, 44.2 (t, $J=2.4 \mathrm{~Hz}$ ), 42.2 (2C), 34.7 ppm.

${ }^{19}$ F NMR (376 MHz, Chloroform- $d$ ) $\delta=-89.12(\mathrm{~d}, J=40.8 \mathrm{~Hz}, 1 \mathrm{~F}),-90.91$ (d, $J=41.4$ $\mathrm{Hz}, 1 \mathrm{~F}) \mathrm{ppm}$.

HRMS (ESI) m/z: [M+Na] $]^{+}$Calcd for $\mathrm{C}_{26} \mathrm{H}_{26} \mathrm{~F}_{2} \mathrm{O}_{2} \mathrm{Na}$ 431.1793; Found 431.1798

2-(3,3-Difluoro-2-(4-methoxyphenyl)allyl)adamantan-2-yl)methanol (3ad)<smiles>COc1ccc(C(CC2(C)C3CC4CC(C3)CC2C4)=C(F)F)cc1</smiles>

The title compound 3ad was isolated as a colorless oil (58 mg, 42\%) through flash chromatography on silica gel eluting with petroleum ether/ethyl acetate (3:1).

${ }^{1} \mathbf{H}$ NMR (400 MHz, Chloroform-d) $\delta=7.26(\mathrm{~d}, J=8.8 \mathrm{~Hz}, 2 \mathrm{H}), 6.88(\mathrm{~d}, J=8.7 \mathrm{~Hz}$, $2 \mathrm{H}), 3.80(\mathrm{~s}, 3 \mathrm{H}), 3.62(\mathrm{~s}, 2 \mathrm{H}), 2.72(\mathrm{t}, J=2.3 \mathrm{~Hz}, 2 \mathrm{H}), 2.08-2.02(\mathrm{~m}, 2 \mathrm{H}), 2.01-1.93$ (m, 2H), 1.87-1.78 (m, 2H), 1.65 (s, 2H), 1.51 (s, 4H), 1.48 (s, 2H) ppm.

${ }^{13}$ C NMR (101 MHz, Chloroform- $d$ ) $\delta=158.8,154.1$ (t, $\left.J=287.8 \mathrm{~Hz}\right), 129.6(\mathrm{t}, J=2.5$ Hz, 2C), 127.2 (dd, J= 5.2, $1.7 \mathrm{~Hz}$ ), 113.9 (2C), 89.9 (dd, $J=21.8,14.6 \mathrm{~Hz}$ ), 64.0, 55.2, 43.9 (t, $J=2.3 \mathrm{~Hz}$ ), 39.6, 32.9 (2C), 32.7 (2C), 31.6 (2C), 30.6, 28.1, 27.7 ppm.

${ }^{19}$ F NMR (376 MHz, Chloroform- $d$ ) $\delta=-90.25$ (d, $\left.J=44.9 \mathrm{~Hz}, 1 \mathrm{~F}\right),-91.19$ (d, $J=44.5$ $\mathrm{Hz}, 1 \mathrm{~F}) \mathrm{ppm}$.

HRMS (ESI) m/z: $[\mathrm{M}+\mathrm{H}]^{+}$Calcd for $\mathrm{C}_{21} \mathrm{H}_{27} \mathrm{~F}_{2} \mathrm{O}_{2}$ 349.1974; Found 349.1980

tert-Butyl 4-(3,3-difluoro-2-(4-methoxyphenyl)allyl)-4-(hydroxymethyl)piperidine-1carboxylate (3ae)<smiles>COc1ccc(C(CC2(C)CCN(C(=O)OC(C)(C)C)CC2O)=C(F)F)cc1</smiles>

The title compound 3ae was isolated as a colorless oil (113 mg, 71\%) through flash chromatography on silica gel eluting with petroleum ether/ethyl acetate (2:1).

${ }^{1}$ H NMR (400 MHz, Chloroform-d) $\delta=7.25(\mathrm{~d}, J=8.8 \mathrm{~Hz}, 2 \mathrm{H}), 6.89(\mathrm{~d}, J=8.7 \mathrm{~Hz}$, $2 \mathrm{H}), 3.81(\mathrm{~s}, 3 \mathrm{H}), 3.33(\mathrm{t}, J=5.9 \mathrm{~Hz}, 4 \mathrm{H}), 3.25(\mathrm{~s}, 2 \mathrm{H}), 2.49$ (t, J=2.4 Hz, 2H), 1.44 (s, 9H), 1.40-1.30 (m, 4H) ppm.

${ }^{13}$ C NMR (101 MHz, Chloroform-d) $\delta=158.9,154.9,154.3$ (dd, $\left.J=289.9,287.4 \mathrm{~Hz}\right)$, 129.4 (t, $J=2.7 \mathrm{~Hz}, 2 \mathrm{C}), 126.7$ (dd, $J=4.8,2.3 \mathrm{~Hz}), 114.1$ (2C), 88.9 (dd, $J=21.7,14.3$ $\mathrm{Hz}), 79.4,66.2,55.2,39.4$ (2C), 38.1 (t, J=2.5 Hz), 32.6, 31.6 (2C), 28.4 (3C) ppm. 
${ }^{19}$ F NMR (376 MHz, Chloroform- $d$ ) $\delta=-89.73(\mathrm{~d}, J=42.4 \mathrm{~Hz}, 1 \mathrm{~F}),-91.47$ (d, $J=42.5$ $\mathrm{Hz}, 1 \mathrm{~F}) \mathrm{ppm}$.

HRMS (ESI) m/z: [M+Na] ${ }^{+}$Calcd for $\mathrm{C}_{21} \mathrm{H}_{29} \mathrm{~F}_{2} \mathrm{NO}_{4} \mathrm{Na}$ 420.1957; Found 420.1965

5,5-Difluoro-4-(4-methoxyphenyl)-2-methyl-2-(phenoxymethyl)pent-4-en-1-ol (3af)<smiles>COc1ccc(C(CC(C)(C)COc2ccccc2)=C(F)F)cc1</smiles>

The title compound 3af was isolated as a colorless oil (100 mg, 72\%) through flash chromatography on silica gel eluting with petroleum ether/ethyl acetate $(3: 1)$.

${ }^{1}$ H NMR (400 MHz, Chloroform- $d$ ) $\delta=7.24-7.18(\mathrm{~m}, 4 \mathrm{H}), 6.93-6.88(\mathrm{~m}, 1 \mathrm{H}), 6.75$ (d, $J=8.8 \mathrm{~Hz}, 2 \mathrm{H}), 6.70(\mathrm{~d}, J=7.7 \mathrm{~Hz}, 2 \mathrm{H}), 3.71(\mathrm{~s}, 3 \mathrm{H}), 3.59(\mathrm{~d}, J=8.9 \mathrm{~Hz}, 1 \mathrm{H}), 3.55(\mathrm{~d}$, $J=8.9 \mathrm{~Hz}, 1 \mathrm{H}), 3.47(\mathrm{~s}, 2 \mathrm{H}), 2.69(\mathrm{dt}, J=14.5,2.7 \mathrm{~Hz}, 1 \mathrm{H}), 2.54-2.44(\mathrm{~m}, 1 \mathrm{H}), 1.84$ (brs, 1H), 0.90 (s, 3H) ppm.

${ }^{13}$ C NMR (101 MHz, Chloroform-d) $\delta=158.7,158.6,154.4(\mathrm{dd}, J=289.7,287.6 \mathrm{~Hz})$, 129.4 (t, $J=2.8 \mathrm{~Hz}, 2 \mathrm{C}$ ), 129.3 (2C), 126.5 (dd, $J=4.4,2.7 \mathrm{~Hz}), 120.8,114.3$ (2C), 113.9 (2C), 88.9 (dd, $J=21.3,14.6 \mathrm{~Hz}), 72.4,68.7,55.2,40.8(\mathrm{t}, J=2.5 \mathrm{~Hz}), 31.8,19.0$ ppm.

${ }^{19}$ F NMR (376 MHz, Chloroform- $d$ ) $\delta=-90.14(\mathrm{~d}, J=42.1 \mathrm{~Hz}, 1 \mathrm{~F}),-91.70(\mathrm{~d}, J=41.6$ $\mathrm{Hz}, 1 \mathrm{~F}) \mathrm{ppm}$.

HRMS (ESI) m/z: [M+Na] ${ }^{+}$Calcd for $\mathrm{C}_{20} \mathrm{H}_{22} \mathrm{~F}_{2} \mathrm{O}_{3} \mathrm{Na} 371.1429$; Found 371.1433

2-Benzyl-2-((benzyloxy)methyl)-5,5-difluoro-4-(4-methoxyphenyl)pent-4-en-1-ol (3ag)<smiles>COc1ccc(C(CC(C)(CO)CBr)=C(F)F)cc1</smiles>

The title compound 3ag was isolated as a colorless oil (116 mg, 66\%) through flash chromatography on silica gel eluting with petroleum ether/ethyl acetate $(3: 1)$.

${ }^{1}$ H NMR (400 MHz, Chloroform-d) $\delta=7.38-7.26(\mathrm{~m}, 3 \mathrm{H}), 7.25-7.16(\mathrm{~m}, 7 \mathrm{H}), 7.14-$ $7.11(\mathrm{~m}, 2 \mathrm{H}), 6.82(\mathrm{~d}, J=8.7 \mathrm{~Hz}, 2 \mathrm{H}), 4.26-4.06(\mathrm{~m}, 2 \mathrm{H}), 3.77(\mathrm{~s}, 3 \mathrm{H}), 3.41-3.26(\mathrm{~m}$, 2H), 3.15-3.03 (m, 2H), 2.82-2.69 (m, 2H), 2.63-2.46 (m, 2H), 2.07 (brs, 1H) ppm.

${ }^{13}$ C NMR (101 MHz, Chloroform- $d$ ) $\delta=158.8,154.2(\mathrm{dd}, J=288.8,288.9 \mathrm{~Hz}), 137.9$, 137.5, 130.6 (2C), 129.5 (t, J=2.8 Hz, 2C), 128.4 (2C), 128.0 (2C), 127.7, 127.6 (2C), $126.7(\mathrm{dd}, J=4.5,2.4 \mathrm{~Hz}), 126.2,113.9$ (2C), 89.1 (dd, $J=21.2,14.5 \mathrm{~Hz}), 73.6,73.2$, 66.8, 55.3, $44.2(\mathrm{t}, J=2.4 \mathrm{~Hz}), 39.4,31.6 \mathrm{ppm}$.

${ }^{19}$ F NMR (376 MHz, Chloroform- $d$ ) $\delta=-89.62(\mathrm{~d}, J=41.4 \mathrm{~Hz}, 1 \mathrm{~F}),-91.21(\mathrm{~d}, J=41.1$ $\mathrm{Hz}, 1 \mathrm{~F}) \mathrm{ppm}$.

HRMS (ESI) m/z: [M+Na] ${ }^{+}$Calcd for $\mathrm{C}_{27} \mathrm{H}_{28} \mathrm{~F}_{2} \mathrm{O}_{3} \mathrm{Na} 461.1899$; Found 461.1903 
2-(2-(4-(tert-Butyl)phenoxy)ethyl)-5,5-difluoro-4-(4-methoxyphenyl)-2-methylpent-4en-1-ol (3ah)<smiles>COc1ccc(C(CC(C)(C)CCOc2ccc(C(C)(C)C)cc2)=C(F)F)cc1</smiles>

The title compound 3ah was isolated as a colorless oil (137 mg, 82\%) through flash chromatography on silica gel eluting with petroleum ether/ethyl acetate $(3: 1)$.

${ }^{1}$ H NMR (400 MHz, Chloroform- $d$ ) $\delta=7.28(\mathrm{~d}, J=8.8 \mathrm{~Hz}, 2 \mathrm{H}), 7.24(\mathrm{~d}, J=8.7 \mathrm{~Hz}$, $2 \mathrm{H}), 6.88(\mathrm{~d}, J=8.8 \mathrm{~Hz}, 2 \mathrm{H}), 6.79(\mathrm{~d}, J=8.8 \mathrm{~Hz}, 2 \mathrm{H}), 4.00-3.86(\mathrm{~m}, 2 \mathrm{H}), 3.80(\mathrm{~s}, 3 \mathrm{H})$, $3.25(\mathrm{~s}, 2 \mathrm{H}), 2.55-2.37(\mathrm{~m}, 2 \mathrm{H}), 2.23(\mathrm{~s}, 1 \mathrm{H}), 1.86-1.73(\mathrm{~m}, 1 \mathrm{H}), 1.71-1.62(\mathrm{~m}, 1 \mathrm{H})$, 1.29 (s, 9H), $0.81(\mathrm{~s}, 3 \mathrm{H}) \mathrm{ppm}$.

${ }^{13}$ C NMR (101 MHz, Chloroform- $d$ ) $\delta=158.8,156.0,154.4(\mathrm{dd}, J=288.8,288.9 \mathrm{~Hz})$, 143.9, 129.5 (t, $J=2.8 \mathrm{~Hz}, 2 \mathrm{C}$ ), 127.1 (dd, $J=4.6,2.5 \mathrm{~Hz}$ ), 126.3 (2C), 113.98 (2C), 113.95 (2C), 89.4 (dd, $J=21.3,14.3 \mathrm{~Hz}), 69.0,64.5,55.2,39.6(\mathrm{t}, J=2.5 \mathrm{~Hz}), 36.3$, 35.5, 34.1, 31.5 (3C), 21.8 ppm.

${ }^{19}$ F NMR (376 MHz, Chloroform- $d$ ) $\delta=-90.01$ (d, $\left.J=42.4 \mathrm{~Hz}, 1 \mathrm{~F}\right),-91.91$ (d, $J=42.3$ $\mathrm{Hz}, 1 \mathrm{~F}) \mathrm{ppm}$.

HRMS (ESI) m/z: [M+Na] ${ }^{+}$Calcd for $\mathrm{C}_{25} \mathrm{H}_{32} \mathrm{~F}_{2} \mathrm{O}_{3} \mathrm{Na}$ 441.2212; Found 441.2215

5,5-Difluoro-2-(2-(4-methoxyphenoxy)ethyl)-4-(4-methoxyphenyl)-2-methylpent-4-en1-ol (3ai)<smiles>COc1ccc(OCCC(C)(C)CC(=C(F)F)c2ccc(OC)cc2)cc1</smiles>

The title compound 3ai was isolated as a colorless oil (119 mg, 76\%) through flash chromatography on silica gel eluting with petroleum ether/ethyl acetate (3:1).

${ }^{1}$ H NMR (400 MHz, Chloroform-d) $\delta=7.24(\mathrm{~d}, J=8.8 \mathrm{~Hz}, 2 \mathrm{H}), 6.88(\mathrm{~d}, J=8.8 \mathrm{~Hz}$, $2 \mathrm{H})$, 6.84-6.74 (m, $4 \mathrm{H}), 3.95-3.89(\mathrm{~m}, 2 \mathrm{H}), 3.79(\mathrm{~s}, 3 \mathrm{H}), 3.75(\mathrm{~s}, 3 \mathrm{H}), 3.25(\mathrm{~s}, 2 \mathrm{H})$, 2.55-2.38 (m, 2H), 2.26 (brs, $1 \mathrm{H}), 1.83-1.71(\mathrm{~m}, 1 \mathrm{H}), 1.70-1.58(\mathrm{~m}, 1 \mathrm{H}), 0.80(\mathrm{~s}, 3 \mathrm{H})$ ppm.

${ }^{13}$ C NMR (101 MHz, Chloroform-d) $\delta=158.8,154.4(\mathrm{dd}, J=289.4,287.5 \mathrm{~Hz}), 154.1$, 152.5, 129.5 (t, $J=2.8 \mathrm{~Hz}, 2 \mathrm{C}$ ), 127.1 (dd, $J=4.6,2.6 \mathrm{~Hz}), 115.5$ (2C), 114.7 (2C), 113.9 (2C), 89.4 (dd, $J=21.4,14.2 \mathrm{~Hz}), 69.0,65.3,55.7,55.2,39.6$ (t, $J=2.5 \mathrm{~Hz}), 36.3$, $35.5,21.9 \mathrm{ppm}$.

${ }^{19}$ F NMR (376 MHz, Chloroform- $d$ ) $\delta=-90.03(\mathrm{~d}, J=42.9 \mathrm{~Hz}, 1 \mathrm{~F}),-91.92(\mathrm{~d}, J=44.2$ $\mathrm{Hz}, 1 \mathrm{~F}) \mathrm{ppm}$.

HRMS (ESI) m/z: [M+Na] ${ }^{+}$Calcd for $\mathrm{C}_{22} \mathrm{H}_{26} \mathrm{~F}_{2} \mathrm{O}_{4} \mathrm{Na}$ 415.1691; Found 415.1689 
1-(4-((6,6-Difluoro-3-(hydroxymethyl)-5-(4-methoxyphenyl)-3-methylhex-5-en-1yl)oxy)phenyl)ethan-1-one (3aj)<smiles>COc1ccc(C(CC(C)(C)CCOc2ccc(C(C)=O)cc2)=C(F)F)cc1</smiles>

The title compound 3aj was isolated as a colorless oil (113 mg, 70\%) through flash chromatography on silica gel eluting with petroleum ether/ethyl acetate $(3: 1)$.

${ }^{1}$ H NMR (400 MHz, Chloroform-d) $\delta=7.91(\mathrm{~d}, J=8.6 \mathrm{~Hz}, 2 \mathrm{H}), 7.26(\mathrm{~d}, J=7.8 \mathrm{~Hz}$, $2 \mathrm{H}), 6.90-6.86(\mathrm{~m}, 4 \mathrm{H}), 4.08-3.99(\mathrm{~m}, 2 \mathrm{H}), 3.79(\mathrm{~s}, 3 \mathrm{H}), 3.26(\mathrm{~s}, 2 \mathrm{H}), 2.55(\mathrm{~s}, 3 \mathrm{H})$, 2.50-2.46 (m, 2H), 1.89-1.78 (m, 2H), 1.78-1.66 (m, 1H), $0.84(\mathrm{~s}, 3 \mathrm{H}) \mathrm{ppm}$.

${ }^{13}$ C NMR (101 MHz, Chloroform-d) $\delta=196.8,162.4,158.8,154.4$ (dd, $J=289.6$, $287.5 \mathrm{~Hz}$ ), 130.6 (2C), 130.5, 129.4 (t, $J=2.7 \mathrm{~Hz}, 2 \mathrm{C}), 126.9$ (dd, $J=4.6,2.5 \mathrm{~Hz}), 114.1$ (2C), 114.0 (2C), 89.3 (dd, $J=21.5,14.3 \mathrm{~Hz}), 68.9,64.9,55.2,39.5$ (t, $J=2.5 \mathrm{~Hz}), 35.9$, $35.3,26.4,21.8 \mathrm{ppm}$.

${ }^{19}$ F NMR (376 MHz, Chloroform- $d$ ) $\delta=-89.91(\mathrm{~d}, J=42.0 \mathrm{~Hz}, 1 \mathrm{~F}),-91.71(\mathrm{~d}, J=41.8$ $\mathrm{Hz}, 1 \mathrm{~F}) \mathrm{ppm}$.

HRMS (ESI) m/z: [M+Na] $]^{+}$Calcd for $\mathrm{C}_{23} \mathrm{H}_{26} \mathrm{~F}_{2} \mathrm{O}_{4} \mathrm{Na}$ 427.1691; Found 427.1699

2-(2-(Benzyloxy)ethyl)-5,5-difluoro-4-(4-methoxyphenyl)-2-methylpent-4-en-1-ol (3ak)<smiles>COc1ccc(C(CC(C)(C)CCOCc2ccccc2)=C(F)F)cc1</smiles>

The title compound 3ak was isolated as a colorless oil (123 mg, 82\%) through flash chromatography on silica gel eluting with petroleum ether/ethyl acetate $(3: 1)$.

${ }^{1}$ H NMR (400 MHz, Chloroform-d) $\delta=7.36-7.29$ (m, 2H), 7.31-7.24 (m, 3H), $7.21(\mathrm{~d}$, $J=8.9 \mathrm{~Hz}, 2 \mathrm{H}), 6.86(\mathrm{~d}, J=8.8 \mathrm{~Hz}, 2 \mathrm{H}), 4.45(\mathrm{~s}, 2 \mathrm{H}), 3.78(\mathrm{~s}, 3 \mathrm{H}), 3.49-3.37(\mathrm{~m}, 2 \mathrm{H})$, $3.18(\mathrm{~s}, 2 \mathrm{H}), 3.05$ (brs, 1H), 2.45-2.30 (m, 2H), 1.66-1.54 (m, 1H), 1.51-1.39 (m, 1H), 0.73 (s, 3H) ppm.

${ }^{13}$ C NMR (101 MHz, Chloroform- $\left.d\right) \delta=158.6,154.3(\mathrm{dd}, J=289.2,287.3 \mathrm{~Hz}), 137.5$, 129.5 (t, $J=2.8 \mathrm{~Hz}, 2 \mathrm{C}), 128.5$ (2C), 127.9, 127.8 (2C), 127.2 (dd, $J=4.7,2.6 \mathrm{~Hz}$ ), 113.9 (2C), 89.5 (dd, $J=21.4,14.0 \mathrm{~Hz}), 73.4,69.3,66.6,55.2,39.7$ (t, $J=2.4 \mathrm{~Hz}), 37.2$, 35.7, $21.9 \mathrm{ppm}$.

${ }^{19}$ F NMR (376 MHz, Chloroform-d) $\delta=-90.13(\mathrm{~d}, J=42.4 \mathrm{~Hz}, 1 \mathrm{~F}),-92.14(\mathrm{~d}, J=42.2$ $\mathrm{Hz}, 1 \mathrm{~F}) \mathrm{ppm}$.

HRMS (ESI) m/z: [M+Na] $]^{+}$Calcd for $\mathrm{C}_{22} \mathrm{H}_{26} \mathrm{~F}_{2} \mathrm{O}_{3} \mathrm{Na}$ 399.1742; Found 399.1749 
2-(2-((tert-Butyldiphenylsilyl)oxy)ethyl)-5,5-difluoro-4-(4-methoxyphenyl)-2-

methylpent-4-en-1-ol (3al)<smiles>COc1ccc(C(CC(C)(C)CCO[Si](c2ccccc2)(c2ccccc2)C(C)(C)C)=C(F)F)cc1</smiles>

The title compound 3al was isolated as a colorless oil (161 mg, 77\%) through flash chromatography on silica gel eluting with petroleum ether/ethyl acetate $(3: 1)$.

${ }^{1}$ H NMR (400 MHz, Chloroform- $d$ ) $\delta=7.65-7.62(\mathrm{~m}, 4 \mathrm{H}), 7.45-7.35(\mathrm{~m}, 6 \mathrm{H}), 7.19(\mathrm{~d}$, $J=8.9 \mathrm{~Hz}, 2 \mathrm{H}), 6.84(\mathrm{~d}, J=8.8 \mathrm{~Hz}, 2 \mathrm{H}), 3.77(\mathrm{~s}, 3 \mathrm{H}), 3.63-3.56(\mathrm{~m}, 2 \mathrm{H}), 3.30(\mathrm{~s}, 2 \mathrm{H})$, 3.13 (brs, 1H), 2.46-2.33 (m, 2H), 1.66-1.51 (m, 1H), 1.49-1.36 (m, 1H), $1.03(\mathrm{~s}, 9 \mathrm{H})$, 0.73 (s, 3H) ppm.

${ }^{13}$ C NMR (101 MHz, Chloroform- $d$ ) $\delta=158.7,154.3(\mathrm{dd}, J=287.9,288.9 \mathrm{~Hz}), 135.6$ (4C), 132.9 (2C), 129.9 (2C), 129.5 (t, $J=2.7 \mathrm{~Hz}, 2 \mathrm{C}$ ), 127.8 (4C), 127.3 (dd, $J=4.5$, $2.5 \mathrm{~Hz}), 113.9$ (2C), 89.5 (dd, $J=21.3,14.0 \mathrm{~Hz}), 69.5,60.7,55.2,40.0,39.8$ (t, $J=2.4$ $\mathrm{Hz}), 35.7,26.8$ (3C), 22.1, $19.0 \mathrm{ppm}$.

${ }^{19}$ F NMR (376 MHz, Chloroform- $d$ ) $\delta=-90.10(\mathrm{~d}, J=43.0 \mathrm{~Hz}, 1 \mathrm{~F}),-92.12$ (d, $J=42.3$ $\mathrm{Hz}, 1 \mathrm{~F}) \mathrm{ppm}$.

HRMS (ESI) m/z: [M+H] $]^{+}$Calcd for $\mathrm{C}_{31} \mathrm{H}_{39} \mathrm{~F}_{2} \mathrm{O}_{3} \mathrm{Si}$ 525.2631; Found 525.2640

6,6-Difluoro-3-(hydroxymethyl)-5-(4-methoxyphenyl)-3-methylhex-5-en-1-yl benzoate (3am)<smiles>COc1ccc(C(CC(C)(C)CCOC(=O)c2ccccc2)=C(F)F)cc1</smiles>

The title compound 3am was isolated as a colorless oil (122 mg, 78\%) through flash chromatography on silica gel eluting with petroleum ether/ethyl acetate $(3: 1)$.

${ }^{1}$ H NMR (400 MHz, Chloroform-d) $\delta=8.03-7.96(\mathrm{~m}, 2 \mathrm{H}), 7.59-7.50(\mathrm{~m}, 1 \mathrm{H})$, 7.47$7.38(\mathrm{~m}, 2 \mathrm{H}), 7.26(\mathrm{~d}, J=10.3 \mathrm{~Hz}, 2 \mathrm{H}), 6.88(\mathrm{~d}, J=8.8 \mathrm{~Hz}, 2 \mathrm{H}), 4.33(\mathrm{t}, J=7.1 \mathrm{~Hz}, 2 \mathrm{H})$, 3.77 (s, 3H), 3.26 (s, 2H), 2.52-2.39 (m, 2H), 1.79-1.68 (m, 2H), 1.60 (brs, 1H), 0.85 (s, 3H) ppm.

${ }^{13}$ C NMR (101 MHz, Chloroform- $\left.d\right) \delta=166.7,158.8,154.4(\mathrm{dd}, J=289.6,287.5 \mathrm{~Hz})$, 132.9, 130.2, 129.5 (2C), 129.4 (t, $J=2.7 \mathrm{~Hz}, 2 \mathrm{C}), 128.4$ (2C), 126.9 (dd, $J=4.7,2.5$ $\mathrm{Hz}), 114.1$ (2C), 89.3 (dd, $J=21.3,14.4 \mathrm{~Hz}), 68.6,61.7,55.2,39.3$ (t, $J=2.5 \mathrm{~Hz}), 35.4$, 34.9, $21.6 \mathrm{ppm}$.

${ }^{19}$ F NMR (376 MHz, Chloroform- $d$ ) $\delta=-89.93(\mathrm{~d}, J=41.8 \mathrm{~Hz}, 1 \mathrm{~F}),-91.72(\mathrm{~d}, J=42.5$ $\mathrm{Hz}, 1 \mathrm{~F}) \mathrm{ppm}$.

HRMS (ESI) m/z: [M+Na] ${ }^{+}$Calcd for $\mathrm{C}_{22} \mathrm{H}_{24} \mathrm{~F}_{2} \mathrm{O}_{4} \mathrm{Na} 413.1535$; Found 413.1542 

cyclohexylpropanoate (3an)<smiles>COc1ccc(C(CC(C)(CO)CCOC(=O)CCC2CCCCC2)=C(F)F)cc1</smiles>

The title compound 3an was isolated as a colorless oil (127 mg, 75\%) through flash chromatography on silica gel eluting with petroleum ether/ethyl acetate $(3: 1)$.

${ }^{1}$ H NMR (400 MHz, Chloroform-d) $\delta=7.24$ (d, J=8.8 Hz, 2H), 6.88 (d, J=8.8 Hz, 2H), $4.07(\mathrm{t}, J=7.1 \mathrm{~Hz}, 2 \mathrm{H}), 3.80(\mathrm{~s}, 3 \mathrm{H}), 3.19(\mathrm{~s}, 2 \mathrm{H}), 2.42(\mathrm{t}, J=2.9 \mathrm{~Hz}, 2 \mathrm{H}), 2.27(\mathrm{t}$, $J=7.6 \mathrm{~Hz}, 2 \mathrm{H}), 1.74-1.53(\mathrm{~m}, 8 \mathrm{H}), 1.54-1.43(\mathrm{~m}, 2 \mathrm{H}), 1.28-1.09$ (m, 4H), 0.99-0.83 (m, $2 \mathrm{H}), 0.78(\mathrm{~s}, 3 \mathrm{H}) \mathrm{ppm}$.

${ }^{13}$ C NMR (101 MHz, Chloroform-d) $\delta=174.2,158.8,154.4$ (dd, $\left.J=289.6,287.4 \mathrm{~Hz}\right)$, 129.4 (t, $J=2.7 \mathrm{~Hz}, 2 \mathrm{C}$ ), 126.9 (dd, $J=4.7,2.5 \mathrm{~Hz}), 114.0$ (2C), 89.2 (dd, $J=21.4,14.3$ $\mathrm{Hz}), 68.5,61.0,55.2,39.2$ (t, $J=2.4 \mathrm{~Hz}), 37.2$, 35.3, 34.9, 32.9 (2C), 32.3, 31.9, 26.5, 26.2 (2C), $21.5 \mathrm{ppm}$.

${ }^{19}$ F NMR (376 MHz, Chloroform- $d$ ) $\delta=-90.00(\mathrm{~d}, J=41.9 \mathrm{~Hz}, 1 \mathrm{~F}),-91.81$ (d, $J=42.2$ $\mathrm{Hz}, 1 \mathrm{~F}) \mathrm{ppm}$.

HRMS (ESI) m/z: [M+Na] ${ }^{+}$Calcd for $\mathrm{C}_{24} \mathrm{H}_{34} \mathrm{~F}_{2} \mathrm{O}_{4} \mathrm{Na}$ 447.2317; Found 447.2327

2-(6,6-Difluoro-3-(hydroxymethyl)-5-(4-methoxyphenyl)-3-methylhex-5-en-1yl)isoindoline-1,3-dione (3ao)<smiles>COc1ccc(C(CC(C)(C)CCN2C(=O)c3ccccc3C2=O)=C(F)F)cc1</smiles>

The title compound 3ao was isolated as a colorless oil (71 mg, 43\%) through flash chromatography on silica gel eluting with petroleum ether/ethyl acetate $(2: 1)$.

${ }^{1}$ H NMR (400 MHz, Chloroform-d) $\delta=7.83(\mathrm{dd}, J=5.5,3.1 \mathrm{~Hz}, 2 \mathrm{H}), 7.70$ (dd, $J=5.5$, $3.1 \mathrm{~Hz}, 2 \mathrm{H}), 7.26(\mathrm{~d}, J=8.7 \mathrm{~Hz}, 2 \mathrm{H}), 6.88(\mathrm{~d}, J=8.6 \mathrm{~Hz}, 2 \mathrm{H}), 3.80(\mathrm{~s}, 3 \mathrm{H}), 3.72-3.61$ (m, 2H), 3.26 (s, 2H), 2.47 (s, 2H), 1.79 (brs, 1H), 1.64 (t, J=8.0 Hz, 2H), $0.83(\mathrm{~s}, 3 \mathrm{H})$ ppm.

${ }^{13}$ C NMR (101 MHz, Chloroform- $d$ ) $\delta=168.3(2 \mathrm{C}), 158.8,154.4(\mathrm{dd}, J=289.5,287.5$ Hz), 133.9 (2C), 132.2 (2C), 129.4 (t, $J=2.7 \mathrm{~Hz}, 2 \mathrm{C}$ ), 126.9 (dd, $J=4.4,2.6 \mathrm{~Hz}$ ), 123.2 (2C), 114.0 (2C), 89.2 (dd, $J=21.3,14.3 \mathrm{~Hz}), 68.1,55.2,39.3$ (t, $J=2.5 \mathrm{~Hz}), 35.4,34.8$, 33.6, $21.2 \mathrm{ppm}$.

${ }^{19}$ F NMR (376 MHz, Chloroform- $d$ ) $\delta=-89.80(\mathrm{~d}, J=42.1 \mathrm{~Hz}, 1 \mathrm{~F}),-91.69(\mathrm{~d}, J=42.1$ $\mathrm{Hz}, 1 \mathrm{~F}) \mathrm{ppm}$.

HRMS (ESI) m/z: [M+Na] ${ }^{+}$Calcd for $\mathrm{C}_{23} \mathrm{H}_{23} \mathrm{~F}_{2} \mathrm{NO}_{4} \mathrm{Na} 438.1487$; Found 438.1491 
<smiles>COc1ccc(C(CC(C)CCc2ccccc2)=C(F)F)cc1</smiles>

The title compound 3ap was isolated as a colorless oil (74 mg, 56\%) through flash chromatography on silica gel eluting with petroleum ether/ethyl acetate $(3: 1)$.

${ }^{1}$ H NMR (400 MHz, Chloroform-d) $\delta=7.28-7.15(\mathrm{~m}, 5 \mathrm{H}), 7.11(\mathrm{~d}, J=8.6 \mathrm{~Hz}, 2 \mathrm{H})$, $6.87(\mathrm{~d}, J=8.8 \mathrm{~Hz}, 2 \mathrm{H}), 3.81$ (s, 3H), 3.61-3.51 (m, 2H), 2.62-2.39 (m, 4H), 1.73-1.54 (m, 4H) ppm.

${ }^{13}$ C NMR (101 MHz, Chloroform- $d$ ) $\delta=158.7,153.8(\mathrm{dd}, J=287.9,286.8 \mathrm{~Hz}), 142.2$, 129.4 (t, $J=3.1 \mathrm{~Hz}, 2 \mathrm{C}$ ), 128.4 (2C), 128.3 (2C), 125.8, 125.6 (t, J=2.9 Hz), 114.0 (2C), 90.4 (dd, $J=20.8,14.6 \mathrm{~Hz}), 64.6,55.3,38.3$ (t, $J=2.4 \mathrm{~Hz}), 33.0,32.3,29.2 \mathrm{ppm}$.

${ }^{19}$ F NMR (376 MHz, Chloroform- $d$ ) $\delta=-91.73(\mathrm{~d}, J=45.9 \mathrm{~Hz}, 1 \mathrm{~F}),-91.92(\mathrm{~d}, J=45.7$ $\mathrm{Hz}, 1 \mathrm{~F}) \mathrm{ppm}$.

HRMS (ESI) m/z: $[\mathrm{M}+\mathrm{H}]^{+}$Calcd for $\mathrm{C}_{20} \mathrm{H}_{23} \mathrm{~F}_{2} \mathrm{O}_{2} 333.1661$; Found 333.1664

trans-2-(3,3-Difluoro-2-(4-methoxyphenyl)allyl)cyclohexan-1-ol (3aq)<smiles>COc1ccc(C(CC2CCCC[C@@H]2O)=C(F)F)cc1</smiles>

The title compound 3aq was isolated as a colorless oil (76 mg, 67\%) through flash chromatography on silica gel eluting with petroleum ether/ethyl acetate (3:1).

${ }^{1} \mathbf{H}$ NMR (500 MHz, Chloroform-d) $\delta=7.28(\mathrm{~d}, J=8.7 \mathrm{~Hz}, 2 \mathrm{H}), 6.89(\mathrm{~d}, J=8.8 \mathrm{~Hz}$, 2H), 3.81 (s, 3H), 3.22 (td, $J=10.0,4.3 \mathrm{~Hz}, 1 \mathrm{H}), 2.93$ (dq, $J=14.3,4.0 \mathrm{~Hz}, 1 \mathrm{H}), 2.17$ (ddd, $J=14.3,10.0,2.3 \mathrm{~Hz}, 1 \mathrm{H}), 2.00-1.88(\mathrm{~m}, 1 \mathrm{H}), 1.76(\mathrm{dt}, J=13.3,3.1 \mathrm{~Hz}, 1 \mathrm{H}), 1.68$ (ddd, $J=13.2,5.8,3.2 \mathrm{~Hz}, 1 \mathrm{H}), 1.59$ (dt, $J=12.7,3.5 \mathrm{~Hz}, 1 \mathrm{H}), 1.40$ (s, 1H), 1.28-1.12 (m, 3H), 1.03 (qt, $J=12.6,3.5 \mathrm{~Hz}, 1 \mathrm{H}), 0.90(\mathrm{qd}, J=12.8,3.5 \mathrm{~Hz}, 1 \mathrm{H}) \mathrm{ppm}$.

${ }^{13}$ C NMR (126 MHz, Chloroform- $d$ ) $\delta=158.6,153.9$ (dd, $\left.J=289.4,285.0 \mathrm{~Hz}\right), 129.4$ (t, $J=3.2 \mathrm{~Hz}, 2 \mathrm{C}), 125.9$ (t, $J=8.8 \mathrm{~Hz}$ ), 113.9 (2C), 90.3 (dd, $J=21.8,13.1 \mathrm{~Hz}$ ), 74.6, $55.2,43.4,35.8,30.5,29.8,25.3,24.9 \mathrm{ppm}$.

${ }^{19}$ F NMR (471 MHz, Chloroform-d) $\delta=-91.92(\mathrm{~d}, J=47.1 \mathrm{~Hz}, 1 \mathrm{~F}),-92.23(\mathrm{~d}, J=47.1$ $\mathrm{Hz}, 1 \mathrm{~F}) \mathrm{ppm}$.

HRMS (ESI) m/z: [M+Na] $]^{+}$Calcd for $\mathrm{C}_{16} \mathrm{H}_{20} \mathrm{~F}_{2} \mathrm{O}_{2} \mathrm{Na}$ 305.1324; Found 305.1337

\section{1-mmol-Scale Synthesis for 3ab}

$\mathrm{TiCp}_{2} \mathrm{Cl}_{2}$ (50 mg, $0.20 \mathrm{mmol}, 20 \mathrm{~mol} \%$ ), $\mathrm{NEt}_{3} \cdot \mathrm{HCl}$ (138 mg, $1.0 \mathrm{mmol}, 1.0$ equiv) and $\mathrm{Zn}$ (195 mg, $3.0 \mathrm{mmol}, 3.0$ equiv) were placed in a Schlenk tube equipped with a stir bar. Then the Schlenk tube was evacuated and filled with nitrogen (three cycles). To 
these solids, 1.0 mL dry DMA, the trifluoromethyl alkenes $1 \mathbf{a}(202 \mathrm{mg}, 1.0 \mathrm{mmol}, 1.0$ equiv) and the epoxide $\mathbf{2 b}$ (144 $\mathrm{mg}, 2.0 \mathrm{mmol}, 2.0$ equiv) were added successively under nitrogen atmosphere. The reaction mixture was stirred at room temperature for 24 hours, before it was quenched by addition of water. The aqueous phase was extracted with ethyl acetate $(3 \times 50 \mathrm{~mL})$, and the combined organic phases were washed with brine, dried over $\mathrm{Na}_{2} \mathrm{SO}_{4}$, filtered and concentrated in vacuo. The crude material was then purified through column chromatography on silica gel (petroleum ether/ethyl acetate= 3:1) to give the gem-difluoroalkenes 3a as a colorless oil (205 $\mathrm{mg}, 80 \%)$.

\section{Procedure for Synthesis of the 6-Fluoro-3,4-Dihydro- $2 H$ -}

\section{Pyrans 4}<smiles>[R]C(CC([R])([R])CO)=C(F)F</smiles>
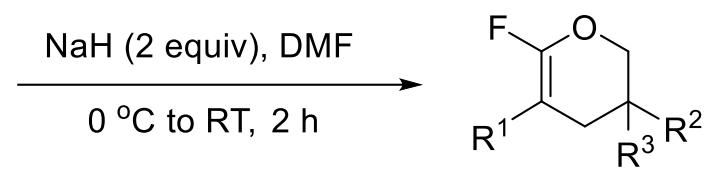

A flask charged with $\mathrm{NaH}(12 \mathrm{mg}, 0.3 \mathrm{mmol}, 2.0$ equiv as a $60 \%$ dispersion in mineral oil) in dry DMF $(1.0 \mathrm{~mL})$ was cooled to $0{ }^{\circ} \mathrm{C}$, before the gem-difluoroalkenes 3 (0.15 mmol, 1.0 equiv) $)^{\mathrm{a}}$ were added. The reaction was allowed to war up and stir at room temperature for 2 hours, before it was quenched with a minimal amount of water. The resultant crude product was purified through column chromatography on silica gel (petroleum ether/ethyl acetate) to give the 6-fluoro-3,4-dihydro-2 $\mathrm{H}$-pyran 4.

${ }^{a} 0.1 \mathrm{mmol}$ 3ca, 3pa and 3aq.

6-Fluoro-3-(4-methoxyphenethyl)-5-(4-methoxyphenyl)-3-methyl-3,4-dihydro-2Hpyran (4aa)<smiles>COc1ccc(CCC2(C)COC(F)=C(c3ccc(OC)cc3)C2)cc1</smiles>

The title compound 4aa was isolated as a colorless oil (45 mg, 85\%) through flash chromatography on silica gel eluting with petroleum ether/ethyl acetate (5:1).

${ }^{1} \mathbf{H}$ NMR (500 MHz, Chloroform-d) $\delta=7.29(\mathrm{~d}, J=9.0 \mathrm{~Hz}, 2 \mathrm{H}), 7.11(\mathrm{~d}, J=8.6 \mathrm{~Hz}$, $2 \mathrm{H}), 6.87(\mathrm{~d}, J=8.9 \mathrm{~Hz}, 2 \mathrm{H}), 6.83(\mathrm{~d}, J=8.7 \mathrm{~Hz}, 2 \mathrm{H}), 3.92(\mathrm{~d}, J=10.2 \mathrm{~Hz}, 1 \mathrm{H}), 3.86(\mathrm{~d}$, $J=11.6 \mathrm{~Hz}, 1 \mathrm{H}), 3.80(\mathrm{~s}, 3 \mathrm{H}), 3.78(\mathrm{~s}, 3 \mathrm{H}), 2.65-2.57(\mathrm{~m}, 2 \mathrm{H}), 2.32(\mathrm{dd}, J=15.4,6.3$ $\mathrm{Hz}, 1 \mathrm{H}), 2.22$ (dd, $J=15.5,5.9 \mathrm{~Hz}, 1 \mathrm{H}), 1.74-1.58(\mathrm{~m}, 2 \mathrm{H}), 1.13(\mathrm{~s}, 3 \mathrm{H}) \mathrm{ppm}$. 
${ }^{13}$ C NMR (126 MHz, Chloroform-d) $\delta=157.9,157.6,153.9$ (d, $\left.J=256.4 \mathrm{~Hz}\right), 134.3$, 129.2 (2C), 128.9 (d, J= 4.4 Hz), 127.9 (d, $J=5.3 \mathrm{~Hz}, 2 \mathrm{C}$ ), 113.9 (2C), 113.7 (2C), 83.3 (d, $J=14.7 \mathrm{~Hz}), 76.1,55.3(2 \mathrm{C}), 40.0,36.9$ (d, $J=3.9 \mathrm{~Hz}), 32.5,29.2,21.7 \mathrm{ppm}$.

${ }^{19}$ F NMR (471 MHz, Chloroform-d) $\delta=-92.67$ (s, 1F) ppm.

HRMS (ESI) m/z: [M+H] ${ }^{+}$Calcd for $\mathrm{C}_{22} \mathrm{H}_{26} \mathrm{FO}_{3} 357.1860$; Found 357.1853

5-(3,5-Dimethoxyphenyl)-6-fluoro-3-(4-methoxyphenethyl)-3-methyl-3,4-dihydro-2Hpyran (4ba)<smiles>COc1ccc(CCC2(C)COC(F)=C(c3cc(OC)cc(OC)c3)C2)cc1</smiles>

The title compound $\mathbf{4 b a}$ was isolated as a colorless oil (46 mg, 79\%) through flash chromatography on silica gel eluting with petroleum ether/ethyl acetate (5:1).

${ }^{1}$ H NMR (400 MHz, Chloroform-d) $\delta=7.10(\mathrm{~d}, J=8.4 \mathrm{~Hz}, 2 \mathrm{H}), 6.83(\mathrm{~d}, J=8.6 \mathrm{~Hz}$, $2 \mathrm{H}), 6.54$ (d, $J=2.3 \mathrm{~Hz}, 2 \mathrm{H}), 6.32$ (t, $J=2.3 \mathrm{~Hz}, 1 \mathrm{H}), 3.93$ (d, $J=10.4 \mathrm{~Hz}, 1 \mathrm{H}), 3.86$ (d, $J=10.3 \mathrm{~Hz}, 1 \mathrm{H}), 3.79(\mathrm{~s}, 6 \mathrm{H}), 3.78(\mathrm{~s}, 3 \mathrm{H}), 2.67-2.54(\mathrm{~m}, 2 \mathrm{H}), 2.33(\mathrm{dd}, J=15.5,6.0$ $\mathrm{Hz}, 1 \mathrm{H}), 2.28-2.17(\mathrm{~m}, 1 \mathrm{H}), 1.70-1.58(\mathrm{~m}, 2 \mathrm{H}), 1.13(\mathrm{~s}, 3 \mathrm{H}) \mathrm{ppm}$.

${ }^{13}$ C NMR (101 MHz, Chloroform- $d$ ) $\delta=160.6$ (2C), 157.9, 154.6 (d, $J=258.4 \mathrm{~Hz}$ ), 138.5 (d, $J=4.1 \mathrm{~Hz}$ ), 134.2, 129.2 (2C), 113.9 (2C), 105.3 (d, J=5.7 Hz, 2C), 97.9, 83.8 (d, $J=13.6 \mathrm{~Hz}), 76.1,55.33$ (2C), 55.29, 39.9, 36.8 (d, $J=3.9 \mathrm{~Hz}), 32.4$ (d, $J=1.3 \mathrm{~Hz})$, 29.1, $21.6 \mathrm{ppm}$.

${ }^{19}$ F NMR (376 MHz, Chloroform- $d$ ) $\delta=-89.25$ (s, 1F) ppm.

HRMS (ESI) m/z: [M+Na] $]^{+}$Calcd for $\mathrm{C}_{23} \mathrm{H}_{27} \mathrm{FO}_{4} \mathrm{Na} 409.1786$; Found 409.1793

6-Fluoro-3-(4-methoxyphenethyl)-5-(2-methoxyphenyl)-3-methyl-3,4-dihydro-2Hpyran (4ca)<smiles>COc1ccc(CCC2(C)COC(F)=C(c3ccccc3OC)C2)cc1</smiles>

The title compound 4ca was isolated as a colorless oil (26 mg, 73\%) through flash chromatography on silica gel eluting with petroleum ether/ethyl acetate (5:1).

${ }^{1}$ H NMR (400 MHz, Chloroform-d) $\delta=7.26-7.14(\mathrm{~m}, 2 \mathrm{H}), 7.12(\mathrm{~d}, J=8.6 \mathrm{~Hz}, 2 \mathrm{H})$, 6.95-6.87 (m, 2H), $6.84(\mathrm{~d}, J=8.6 \mathrm{~Hz}, 2 \mathrm{H}), 3.96(\mathrm{~d}, J=10.3 \mathrm{~Hz}, 1 \mathrm{H}), 3.90(\mathrm{~d}, J=10.4$ $\mathrm{Hz}, 1 \mathrm{H}), 3.81$ (s, 3H), 3.79 (s, 3H), 2.66-2.56 (m, 2H), 2.32 (dd, J= 15.8, 5.9 Hz, 1H), 2.17 (dd, $J=15.7,5.8 \mathrm{~Hz}, 1 \mathrm{H}), 1.76-1.62$ (m, 2H), 1.13 (s, 3H) ppm.

${ }^{13}$ C NMR (101 MHz, Chloroform-d) $\delta=157.8,157.4,153.2(\mathrm{~d}, J=252.2 \mathrm{~Hz}), 134.5$, 131.2 (d, $J=2.0 \mathrm{~Hz}$ ), 129.2 (2C), 128.2, 125.4 (d, $J=1.5 \mathrm{~Hz}$ ), 120.4, 113.9 (2C), 111.0, 
$81.6(\mathrm{~d}, J=18.4 \mathrm{~Hz}), 76.5,55.5,55.3,39.8,36.9$ (d, $J=3.4 \mathrm{~Hz}), 32.3(\mathrm{~d}, J=1.3 \mathrm{~Hz})$, 29.2, $21.5 \mathrm{ppm}$.

${ }^{19}$ F NMR (376 MHz, Chloroform- $d$ ) $\delta=-93.69$ (s, 1F) ppm.

HRMS (ESI) m/z: [M+H] $]^{+}$Calcd for $\mathrm{C}_{22} \mathrm{H}_{26} \mathrm{FO}_{3} 357.1860$; Found 357.1864

5-(4-(tert-Butyl)phenyl)-6-fluoro-3-(4-methoxyphenethyl)-3-methyl-3,4-dihydro-2Hpyran (4fa)<smiles>COc1ccc(CCC2(C)COC(F)=C(c3ccc(CC(C)C)cc3)C2)cc1</smiles>

The title compound $\mathbf{4 f a}$ was isolated as a colorless oil (50 $\mathrm{mg}, 87 \%)$ through flash chromatography on silica gel eluting with petroleum ether/ethyl acetate (5:1).

${ }^{1}$ H NMR (500 MHz, Chloroform-d) $\delta=7.35(\mathrm{~d}, J=8.6 \mathrm{~Hz}, 2 \mathrm{H}), 7.32(\mathrm{~d}, J=8.4 \mathrm{~Hz}$, $2 \mathrm{H}), 7.10(\mathrm{~d}, J=8.5 \mathrm{~Hz}, 2 \mathrm{H}), 6.83(\mathrm{~d}, J=8.5 \mathrm{~Hz}, 2 \mathrm{H}), 3.92(\mathrm{~d}, J=10.2 \mathrm{~Hz}, 1 \mathrm{H}), 3.86$ (d, $J=10.2 \mathrm{~Hz}, 1 \mathrm{H}), 3.78(\mathrm{~s}, 3 \mathrm{H}), 2.66-2.56(\mathrm{~m}, 2 \mathrm{H}), 2.36(\mathrm{dd}, J=15.5,6.0 \mathrm{~Hz}, 1 \mathrm{H}), 2.25$ $(\mathrm{dd}, J=15.5,5.9 \mathrm{~Hz}, 1 \mathrm{H}), 1.71-1.58(\mathrm{~m}, 2 \mathrm{H}), 1.31(\mathrm{~s}, 9 \mathrm{H}), 1.12(\mathrm{~s}, 3 \mathrm{H}) \mathrm{ppm}$.

${ }^{13}$ C NMR (126 MHz, Chloroform-d) $\delta=157.9,154.3$ (d, $\left.J=257.4 \mathrm{~Hz}\right), 148.6,134.3$, 133.5 (d, $J=4.4 \mathrm{~Hz}$ ), 129.2 (2C), 126.5 (d, J=4.8 Hz, 2C), 125.2 (2C), 113.9 (2C), 83.5 (d, $J=13.9 \mathrm{~Hz}), 76.2,55.3,40.0,36.6$ (d, $J=3.8 \mathrm{~Hz}), 34.4,32.4,31.4$ (3C), 29.2, 21.7 ppm.

${ }^{19}$ F NMR (471 MHz, Chloroform- $d$ ) $\delta=-91.28$ (s, 1F) ppm.

HRMS (ESI) m/z: [M+H] ${ }^{+}$Calcd for $\mathrm{C}_{25} \mathrm{H}_{32} \mathrm{FO}_{2} 383.2381$; Found 383.2392

5-(3-Chloro-4-methoxyphenyl)-6-fluoro-3-(4-methoxyphenethyl)-3-methyl-3,4dihydro-2H-pyran (4ha)<smiles>COc1ccc(CCC2(C)COC(F)=C(c3ccc(OC)c(Cl)c3)C2)cc1</smiles>

The title compound 4 ha was isolated as a colorless oil (45 mg, 78\%) through flash chromatography on silica gel eluting with petroleum ether/ethyl acetate $(5: 1)$.

${ }^{1}$ H NMR (500 MHz, Chloroform- $d$ ) $\delta=7.52(\mathrm{~d}, J=2.3 \mathrm{~Hz}, 1 \mathrm{H}), 7.28(\mathrm{~d}, J=8.0 \mathrm{~Hz}$, $1 \mathrm{H}), 7.11(\mathrm{~d}, J=8.2 \mathrm{~Hz}, 2 \mathrm{H}), 6.85(\mathrm{~d}, J=8.5 \mathrm{~Hz}, 2 \mathrm{H}), 6.83-6.81(\mathrm{~m}, 1 \mathrm{H}), 3.93(\mathrm{~d}, J=$ $10.3 \mathrm{~Hz}, 1 \mathrm{H}), 3.88$ (s, 3H), $3.86(\mathrm{~d}, J=10.3 \mathrm{~Hz}, 1 \mathrm{H}), 3.78$ (s, 3H), 2.66-2.59 (m, 2H), $2.29(\mathrm{dd}, J=15.5,6.1 \mathrm{~Hz}, 1 \mathrm{H}), 2.19(\mathrm{dd}, J=15.4,6.0 \mathrm{~Hz}, 1 \mathrm{H}), 1.72-1.58(\mathrm{~m}, 2 \mathrm{H}), 1.13$ (s, 3H) ppm.

${ }^{13}$ C NMR (126 MHz, Chloroform-d) $\delta=157.9,154.3$ (d, $\left.J=257.4 \mathrm{~Hz}\right), 153.8,134.1$, 131.5 (d, $J=4.8 \mathrm{~Hz}), 130.4$ (d, $J=3.9 \mathrm{~Hz}), 129.2$ (2C), 127.0 (d, $J=6.2 \mathrm{~Hz}), 113.9$ (2C), 
111.6, 111.4, 82.4 (d, $J=14.6 \mathrm{~Hz}), 76.1,56.3,55.3,39.9,36.7$ (d, $J=3.9 \mathrm{~Hz}), 32.5,29.1$, $21.7 \mathrm{ppm}$.

${ }^{19}$ F NMR (471 MHz, Chloroform-d) $\delta=-91.09$ (s,1F) ppm.

HRMS (ESI) m/z: $[\mathrm{M}+\mathrm{H}]^{+}$Calcd for $\mathrm{C}_{22} \mathrm{H}_{25} \mathrm{FClO}_{3} 391.1471$; Found 391.1476

5-(4-Chlorophenyl)-6-fluoro-3-(4-methoxyphenethyl)-3-methyl-3,4-dihydro-2H-pyran (4ja)<smiles>COc1ccc(CCC2(C)COC(F)=C(c3ccc(Cl)cc3)C2)cc1</smiles>

The title compound $\mathbf{4 j a}$ was isolated as a colorless oil (43 $\mathrm{mg}, 80 \%)$ through flash chromatography on silica gel eluting with petroleum ether/ethyl acetate $(5: 1)$.

${ }^{1}$ H NMR (500 MHz, Chloroform-d) $\delta=7.30-7.25(\mathrm{~m}, 4 \mathrm{H}), 7.10(\mathrm{~d}, J=8.6 \mathrm{~Hz}, 2 \mathrm{H})$, $6.83(\mathrm{~d}, J=8.6 \mathrm{~Hz}, 2 \mathrm{H}), 3.94(\mathrm{~d}, J=10.3 \mathrm{~Hz}, 1 \mathrm{H}), 3.87(\mathrm{~d}, J=10.4 \mathrm{~Hz}, 1 \mathrm{H}), 3.78(\mathrm{~s}$, $3 \mathrm{H}), 2.67-2.54(\mathrm{~m}, 2 \mathrm{H}), 2.31(\mathrm{dd}, J=15.6,6.1 \mathrm{~Hz}, 1 \mathrm{H}), 2.22(\mathrm{dd}, J=15.4,5.9 \mathrm{~Hz}, 1 \mathrm{H})$, 1.74-1.56 (m, 2H), 1.13 (s, 3H) ppm.

${ }^{13}$ C NMR (126 MHz, Chloroform- $d$ ) $\delta=157.9,154.6$ (d, $\left.J=258.3 \mathrm{~Hz}\right), 134.9$ (d, $J=$ $4.5 \mathrm{~Hz}$ ), 134.1, 131.2, 129.2 (2C), 128.3 (2C), 128.0 (d, J=6.2 Hz, 2C), 113.9 (2C), 82.9 (d, $J=13.8 \mathrm{~Hz}), 76.2,55.3,39.9,36.6$ (d, $J=4.0 \mathrm{~Hz}), 32.4,29.1,21.7$ ppm.

${ }^{19}$ F NMR (471 MHz, Chloroform-d) $\delta=-89.86$ (s, 1F) ppm.

HRMS (ESI) $\mathrm{m} / \mathrm{z}$ : $[\mathrm{M}+\mathrm{H}]^{+}$Calcd for $\mathrm{C}_{21} \mathrm{H}_{23} \mathrm{FClO}_{2} 361.1365$; Found 361.1383

2-(4-(6-Fluoro-3-(4-methoxyphenethyl)-3-methyl-3,4-dihydro-2H-pyran-5-

yl)phenyl)pyridine (4ka)<smiles>COc1ccc(CCC2(C)COC(F)=C(c3ccc(-c4ccccn4)cc3)C2)cc1</smiles>

The title compound 4ka was isolated as a colorless oil (51 mg, 84\%) through flash chromatography on silica gel eluting with petroleum ether/ethyl acetate (5:1).

${ }^{1}$ H NMR (400 MHz, Chloroform-d) $\delta=8.68(\mathrm{~d}, J=4.8 \mathrm{~Hz}, 1 \mathrm{H}), 7.96(\mathrm{~d}, J=8.5 \mathrm{~Hz}$, $2 \mathrm{H}), 7.72(\mathrm{~d}, J=4.9 \mathrm{~Hz}, 2 \mathrm{H}), 7.49(\mathrm{~d}, J=8.6 \mathrm{~Hz}, 2 \mathrm{H}), 7.21-7.16(\mathrm{~m}, 1 \mathrm{H}), 7.12(\mathrm{~d}, J=$ $8.6 \mathrm{~Hz}, 2 \mathrm{H}), 6.83(\mathrm{~d}, J=8.6 \mathrm{~Hz}, 2 \mathrm{H}), 3.96(\mathrm{~d}, J=10.3 \mathrm{~Hz}, 1 \mathrm{H}), 3.89$ (d, $J=10.3 \mathrm{~Hz}$, $1 \mathrm{H}), 3.78(\mathrm{~s}, 3 \mathrm{H}), 2.68-2.58(\mathrm{~m}, 2 \mathrm{H}), 2.40(\mathrm{dd}, J=15.4,6.0 \mathrm{~Hz}, 1 \mathrm{H}), 2.31$ (dd, $J=15.4$, $5.9 \mathrm{~Hz}, 1 \mathrm{H}), 1.72-1.60(\mathrm{~m}, 2 \mathrm{H}), 1.15$ (s, 3H) ppm.

${ }^{13}$ C NMR (101 MHz, Chloroform- $d$ ) $\delta=157.9,157.1,154.8(\mathrm{~d}, J=259.0 \mathrm{~Hz}), 149.7$, 137.2 (d, J=4.6 Hz), 136.7, 136.5, 134.1, 129.2 (2C), 126.9 (d, J=5.9 Hz, 2C), 126.6 
(2C), 121.9, 120.3, 113.9 (2C), 83.5 (d, $J=13.6 \mathrm{~Hz}), 76.2,55.3,39.9,36.5$ (d, $J=4.0$ $\mathrm{Hz}), 32.4$ (d, $J=1.4 \mathrm{~Hz}), 29.2,21.7 \mathrm{ppm}$.

${ }^{19}$ F NMR (376 MHz, Chloroform- $d$ ) $\delta=-89.07$ (s, 1F) ppm.

HRMS (ESI) m/z: $[\mathrm{M}+\mathrm{H}]^{+}$Calcd for $\mathrm{C}_{26} \mathrm{H}_{27} \mathrm{FNO}_{2} 404.2020$; Found 404.2039

5-(6-Fluoro-3-(4-methoxyphenethyl)-3-methyl-3,4-dihydro-2H-pyran-5-yl)-2-

methoxypyridine (4na)<smiles>COc1ccc(CCC2(C)COC(F)=C(c3ccc(OC)nc3)C2)cc1</smiles>

The title compound 4na was isolated as a colorless oil (43 mg, 81\%) through flash chromatography on silica gel eluting with petroleum ether/ethyl acetate $(5: 1)$.

${ }^{1}$ H NMR (500 MHz, Chloroform- $d$ ) $\delta=8.13(\mathrm{~s}, 1 \mathrm{H}), 7.60(\mathrm{dd}, J=8.7,2.5 \mathrm{~Hz}, 1 \mathrm{H})$, $7.11(\mathrm{~d}, J=8.4 \mathrm{~Hz}, 2 \mathrm{H}), 6.83(\mathrm{~d}, J=8.6 \mathrm{~Hz}, 2 \mathrm{H}), 6.71(\mathrm{~d}, J=8.7 \mathrm{~Hz}, 1 \mathrm{H}), 3.95(\mathrm{~d}, J=$ $10.6 \mathrm{~Hz}, 1 \mathrm{H}), 3.93$ (s, 3H), 3.88 (d, J=10.5 Hz, 1H), 3.78 (s, 3H), 2.65-2.55 (m, 2H), $2.31(\mathrm{dd}, J=15.4,6.1 \mathrm{~Hz}, 1 \mathrm{H}), 2.21(\mathrm{dd}, J=15.4,6.0 \mathrm{~Hz}, 1 \mathrm{H}), 1.73-1.58(\mathrm{~m}, 2 \mathrm{H}), 1.14$ (s, 3H) ppm.

${ }^{13}$ C NMR (126 MHz, Chloroform- $d$ ) $\delta=162.2,157.9,154.3$ (d, $\left.J=256.6 \mathrm{~Hz}\right), 144.5$ (d, J=4.7 Hz), 137.4 (d, J=6.0 Hz), 134.1, $129.2(2 \mathrm{C}), 125.4$ (d, $J=3.8 \mathrm{~Hz}), 113.9(2 \mathrm{C})$, 110.3, 80.7 (d, $J=14.8 \mathrm{~Hz}), 76.2,55.3,53.4,39.9,36.4$ (d, J=3.9 Hz), 32.4, 29.1, 21.7 ppm.

${ }^{19}$ F NMR (471 MHz, Chloroform- $d$ ) $\delta=-91.28(\mathrm{~s}, 1 \mathrm{~F}) \mathrm{ppm}$.

HRMS (ESI) m/z: $[\mathrm{M}+\mathrm{H}]^{+}$Calcd for $\mathrm{C}_{21} \mathrm{H}_{25} \mathrm{FNO}_{3}$ 358.1813; Found 358.1818

5-(Benzo[b]thiophen-3-yl)-6-fluoro-3-(4-methoxyphenethyl)-3-methyl-3,4-dihydro2H-pyran (4oa)<smiles>COc1ccc(CCC2(C)COC(F)=C(c3csc4ccccc34)C2)cc1</smiles>

The title compound 40 a was isolated as a colorless oil (31 mg, 82\%) through flash chromatography on silica gel eluting with petroleum ether/ethyl acetate $(5: 1)$.

${ }^{1}$ H NMR (400 MHz, Chloroform-d) $\delta=7.85(\mathrm{~d}, J=9.4 \mathrm{~Hz}, 1 \mathrm{H}), 7.71(\mathrm{~d}, J=7.9 \mathrm{~Hz}$, $1 \mathrm{H}), 7.41-7.32(\mathrm{~m}, 2 \mathrm{H}), 7.21(\mathrm{~s}, 1 \mathrm{H}), 7.12(\mathrm{~d}, J=8.6 \mathrm{~Hz}, 2 \mathrm{H}), 6.84(\mathrm{~d}, J=8.6 \mathrm{~Hz}, 2 \mathrm{H})$, $4.03(\mathrm{~d}, J=10.4 \mathrm{~Hz}, 1 \mathrm{H}), 3.96(\mathrm{~d}, J=10.4 \mathrm{~Hz}, 1 \mathrm{H}), 3.79$ (s, 3H), 2.71-2.54 (m, 2H), 2.37 (dd, $J=15.9,6.0 \mathrm{~Hz}, 1 \mathrm{H}), 2.27$ (dd, $J=16.2,5.5 \mathrm{~Hz}, 1 \mathrm{H}), 1.76-1.63(\mathrm{~m}, 2 \mathrm{H}), 1.20$ (s, 3H) ppm.

${ }^{13}$ C NMR (101 MHz, Chloroform-d) $\delta=157.9,154.1$ (d, $\left.J=254.8 \mathrm{~Hz}\right), 139.9,138.1$, 134.1, 132.1 (d, $J=1.6 \mathrm{~Hz}), 129.2$ (2C), 124.2, 123.9, 123.6, 122.9 (d, $J=3.4 \mathrm{~Hz}), 122.8$, 
113.9 (2C), 78.6 (d, $J=18.7 \mathrm{~Hz}), 76.5,55.3,39.9,38.3$ (d, $J=3.7 \mathrm{~Hz}), 32.6,29.2,21.6$ ppm.

${ }^{19}$ F NMR (376 MHz, Chloroform- $d$ ) $\delta=-88.89$ (s, 1F) ppm.

HRMS (ESI) $\mathrm{m} / \mathrm{z}$ : $[\mathrm{M}+\mathrm{H}]^{+}$Calcd for $\mathrm{C}_{23} \mathrm{H}_{24} \mathrm{FO}_{2} \mathrm{~S}$ 383.1476; Found 383.1479

6-Fluoro-5-(4-methoxyphenyl)-3,3-dimethyl-3,4-dihydro-2H-pyran (4ab)<smiles>COc1ccc(C2=C(F)OCC(C)(C)C2)cc1</smiles>

The title compound 4ab was isolated as a colorless oil (29 $\mathrm{mg}, 83 \%)$ through flash chromatography on silica gel eluting with petroleum ether/ethyl acetate (5:1).

${ }^{1} \mathbf{H}$ NMR (500 MHz, Chloroform-d) $\delta=7.30(\mathrm{~d}, J=9.0 \mathrm{~Hz}, 2 \mathrm{H}), 6.87(\mathrm{~d}, J=8.8 \mathrm{~Hz}$, $2 \mathrm{H}), 3.81(\mathrm{~s}, 2 \mathrm{H}), 3.80(\mathrm{~s}, 3 \mathrm{H}), 2.21(\mathrm{~d}, J=6.0 \mathrm{~Hz}, 2 \mathrm{H}), 1.08(\mathrm{~s}, 6 \mathrm{H}) \mathrm{ppm}$.

${ }^{13}$ C NMR (126 MHz, Chloroform- $d$ ) $\delta=157.5,153.8$ (d, $\left.J=256.3 \mathrm{~Hz}\right), 128.9$ (d, $J=$ $3.8 \mathrm{~Hz}), 127.9$ (d, $J=5.4 \mathrm{~Hz}, 2 \mathrm{C}), 113.7$ (2C), 83.4 (d, $J=14.6 \mathrm{~Hz}), 77.4,55.3,38.3$ (d, $J=3.8 \mathrm{~Hz}), 29.6,24.8(2 \mathrm{C}) \mathrm{ppm}$.

${ }^{19}$ F NMR (471 MHz, Chloroform-d) $\delta=-92.96$ (s, 1F) ppm.

HRMS (ESI) m/z: [M+Na] ${ }^{+}$Calcd for $\mathrm{C}_{14} \mathrm{H}_{17} \mathrm{FO}_{2} \mathrm{Na} 259.1105$; Found 259.1112

tert-Butyl 3-fluoro-4-(4-methoxyphenyl)-2-oxa-9-azaspiro[5.5] undec-3-ene-9-carboxy -late (4ae)<smiles>COc1ccc(C2=C(F)OCC3(CCN(C(=O)OCc4ccccc4)CC3)C2)cc1</smiles>

The title compound 4ae was isolated as a colorless oil (46 mg, 81\%) through flash chromatography on silica gel eluting with petroleum ether/ethyl acetate (5:1).

${ }^{1}$ H NMR (400 MHz, Chloroform-d) $\delta=7.29(\mathrm{~d}, J=9.0 \mathrm{~Hz}, 2 \mathrm{H}), 6.87(\mathrm{~d}, J=8.9 \mathrm{~Hz}$, $2 \mathrm{H}), 3.95(\mathrm{~s}, 2 \mathrm{H}), 3.81(\mathrm{~s}, 3 \mathrm{H}), 3.62-3.53(\mathrm{~m}, 2 \mathrm{H}), 3.41-3.24(\mathrm{~m}, 2 \mathrm{H}), 2.33(\mathrm{~d}, J=6.0$ $\mathrm{Hz}, 2 \mathrm{H}), 1.60-1.44$ (m, 4H), 1.47 (s, 9H) ppm.

${ }^{13}$ C NMR (101 MHz, Chloroform- $d$ ) $\delta=157.7,154.8,153.7$ (d, $\left.J=257.0 \mathrm{~Hz}\right), 128.5$ (d, $J=4.0 \mathrm{~Hz}$ ), 127.9 (d, $J=5.1 \mathrm{~Hz}, 2 \mathrm{C}), 113.7$ (2C), 82.8 (d, $J=14.8 \mathrm{~Hz}), 79.7,74.9$, 55.3, 39.2 (2C), 34.5 (d, $J=4.0 \mathrm{~Hz}), 32.3(2 \mathrm{C}), 31.1$ (d, $J=1.4 \mathrm{~Hz}), 28.5$ (3C) ppm.

${ }^{19}$ F NMR (376 MHz, Chloroform-d) $\delta=-92.15$ (s, 1F) ppm.

HRMS (ESI) m/z: [M+Na] ${ }^{+}$Calcd for $\mathrm{C}_{21} \mathrm{H}_{28} \mathrm{FNO}_{4} \mathrm{Na} 400.1895$; Found 400.1902 
6-Fluoro-3-(2-(4-methoxyphenoxy)ethyl)-5-(4-methoxyphenyl)-3-methyl-3,4-dihydro$2 H$-pyran (4ai)<smiles>COc1ccc(OCCC2(C)COC(F)=C(c3ccc(OC)cc3)C2)cc1</smiles>

The title compound 4ai was isolated as a colorless oil (42 $\mathrm{mg}, 76 \%)$ through flash chromatography on silica gel eluting with petroleum ether/ethyl acetate (5:1).

${ }^{1}$ H NMR (500 MHz, Chloroform-d) $\delta=7.29(\mathrm{~d}, J=9.0 \mathrm{~Hz}, 2 \mathrm{H}), 6.87(\mathrm{~d}, J=8.9 \mathrm{~Hz}$, 2H), 6.87-6.75 (m, 4H), $4.06(\mathrm{t}, J=6.4 \mathrm{~Hz}, 2 \mathrm{H}), 4.00-3.93(\mathrm{~m}, 2 \mathrm{H}), 3.80(\mathrm{~s}, 3 \mathrm{H}), 3.77$ (s, 3H), $2.41(\mathrm{dd}, J=15.6,6.1 \mathrm{~Hz}, 1 \mathrm{H}), 2.22(\mathrm{dd}, J=15.6,5.9 \mathrm{~Hz}, 1 \mathrm{H}), 1.88(\mathrm{t}, J=6.4$ $\mathrm{Hz}, 2 \mathrm{H}), 1.16$ (s, 3H) ppm.

${ }^{13}$ C NMR (126 MHz, Chloroform- $d$ ) $\delta=157.6,153.9,153.8$ (d, $\left.J=259.0 \mathrm{~Hz}\right), 152.8$, 128.7 (d, $J=4.4 \mathrm{~Hz}$ ), 127.9 (d, $J=5.1 \mathrm{~Hz}, 2 \mathrm{C}$ ), 115.3 (2C), 114.7 (2C), 113.7 (2C), 83.2 (d, $J=14.7 \mathrm{~Hz}), 76.2,64.7,55.8,55.3,37.3$ (d, $J=3.8 \mathrm{~Hz}), 36.6,31.9,22.1 \mathrm{ppm}$.

${ }^{19}$ F NMR (471 MHz, Chloroform-d) $\delta=-92.58$ (s, 1F) ppm.

HRMS (ESI) m/z: [M+Na] $]^{+}$Calcd for $\mathrm{C}_{22} \mathrm{H}_{25} \mathrm{FO}_{4} \mathrm{Na} 395.1629$; Found 395.1634

3-(2-(Benzyloxy)ethyl)-6-fluoro-5-(4-methoxyphenyl)-3-methyl-3,4-dihydro-2H-pyran (4ak)<smiles>COc1ccc(C2=C(F)OCC(C)(CCOCc3ccccc3)C2)cc1</smiles>

The title compound 4ak was isolated as a colorless oil (38 mg, 71\%) through flash chromatography on silica gel eluting with petroleum ether/ethyl acetate (5:1).

${ }^{1}$ H NMR (400 MHz, Chloroform- $d$ ) $\delta=7.37-7.30$ (m, 4H), 7.29-7.25 (m, 3H), 6.85 (d, $J=8.9 \mathrm{~Hz}, 2 \mathrm{H}), 4.50(\mathrm{~s}, 2 \mathrm{H}), 3.91(\mathrm{~s}, 2 \mathrm{H}), 3.80(\mathrm{~s}, 3 \mathrm{H}), 3.63(\mathrm{t}, J=6.6 \mathrm{~Hz}, 2 \mathrm{H}), 2.36$ (dd, $J=15.6,6.1 \mathrm{~Hz}, 1 \mathrm{H}), 2.15(\mathrm{dd}, J=15.2,5.5 \mathrm{~Hz}, 1 \mathrm{H}), 1.73(\mathrm{t}, J=6.6 \mathrm{~Hz}, 2 \mathrm{H}), 1.09$ (s, 3H) ppm.

${ }^{13}$ C NMR (101 MHz, Chloroform- $d$ ) $\delta=157.54,157.53,153.8(\mathrm{~d}, J=256.1 \mathrm{~Hz}), 138.2$, 128.8 (d, $J=3.9 \mathrm{~Hz}), 128.4(2 \mathrm{C}), 127.9$ (d, $J=5.2 \mathrm{~Hz}, 2 \mathrm{C}), 127.6$ (2C), 113.6 (2C), $83.2(\mathrm{~d}, J=14.6 \mathrm{~Hz}), 76.3,73.2,66.6,55.3,37.2(\mathrm{~d}, J=4.0 \mathrm{~Hz}), 37.1,31.8(\mathrm{~d}, J=1.4$ $\mathrm{Hz}), 21.9 \mathrm{ppm}$.

${ }^{19}$ F NMR (376 MHz, Chloroform-d) $\delta=-92.66$ (s, 1F) ppm.

HRMS (ESI) m/z: $[\mathrm{M}+\mathrm{H}]^{+}$Calcd for $\mathrm{C}_{22} \mathrm{H}_{26} \mathrm{FO}_{3} 357.1860$; Found 357.1865 
<smiles>COc1ccc(C2=C(F)OCC(CCc3ccccc3)C2)cc1</smiles>

The title compound 4ap was isolated as a colorless oil (20 mg, 65\%) through flash chromatography on silica gel eluting with petroleum ether/ethyl acetate (10:1).

${ }^{1}$ H NMR (500 MHz, Chloroform-d) $\delta=7.33-7.28(\mathrm{~m}, 4 \mathrm{H}), 7.20(\mathrm{~d}, J=7.9 \mathrm{~Hz}, 3 \mathrm{H})$, $6.87(\mathrm{~d}, J=8.8 \mathrm{~Hz}, 2 \mathrm{H}), 4.25$ (dd, $J=10.3,3.5 \mathrm{~Hz}, 1 \mathrm{H}), 3.90(\mathrm{t}, J=9.7 \mathrm{~Hz}, 1 \mathrm{H}), 3.81$ $(\mathrm{s}, 3 \mathrm{H}), 2.79-2.69(\mathrm{~m}, 2 \mathrm{H}), 2.60-2.53(\mathrm{~m}, 1 \mathrm{H}), 2.22-2.16(\mathrm{~m}, 1 \mathrm{H}), 2.12-2.03(\mathrm{~m}$, $1 \mathrm{H}), 1.77-1.64(\mathrm{~m}, 2 \mathrm{H})$.

${ }^{13}$ C NMR (126 MHz, Chloroform- $d$ ) $\delta=157.5,154.7$ (d, $\left.J=255.8 \mathrm{~Hz}\right), 141.6,128.8$ (d, $J=4.1 \mathrm{~Hz}), 128.5$ (2C), 128.3 (2C), 127.9 (d, $J=5.1 \mathrm{~Hz}, 2 \mathrm{C}), 126.1,113.6$ (2C), 83.7 (d, $J=14.3 \mathrm{~Hz}), 72.2,55.3,33.2,33.0,32.2,30.4$ (d, $J=3.7 \mathrm{~Hz}) \mathrm{ppm}$.

${ }^{19}$ F NMR (471 MHz, Chloroform-d) $\delta=-91.36$ (s, 1F) ppm.

HRMS (ESI) $\mathrm{m} / \mathrm{z}$ : [M+Na] $]^{+}$Calcd for $\mathrm{C}_{20} \mathrm{H}_{21} \mathrm{FO}_{2} \mathrm{Na} 335.1418$; Found 335.1423

trans-2-Fluoro-3-(4-methoxyphenyl)-4a,5,6,7,8,8a-hexahydro-4H-chromene (4aq)<smiles>COc1ccc(C2=C(F)OC3CCCC[C@H]3C2)cc1</smiles>

The title compound 4aq was isolated as a colorless oil (29 mg, 74\%) through flash chromatography on silica gel eluting with petroleum ether/ethyl acetate (8:1).

${ }^{1}$ H NMR (400 MHz, Chloroform-d) $\delta=7.30(\mathrm{~d}, J=9.0 \mathrm{~Hz}, 2 \mathrm{H}), 6.86(\mathrm{~d}, J=8.9 \mathrm{~Hz}$, $2 \mathrm{H}), 3.80(\mathrm{~s}, 3 \mathrm{H}), 3.75$ (dd, $J=10.7,4.5 \mathrm{~Hz}, 1 \mathrm{H}), 2.38$ (dt, $J=15.4,5.5 \mathrm{~Hz}, 1 \mathrm{H}), 2.22$ $2.08(\mathrm{~m}, 2 \mathrm{H}), 1.98(\mathrm{dt}, J=13.8,3.0 \mathrm{~Hz}, 1 \mathrm{H}), 1.87(\mathrm{dq}, J=9.4,3.2 \mathrm{~Hz}, 1 \mathrm{H}), 1.77-1.66$ (m, 2H), 1.54-1.44 (m, 1H), 1.38-1.28 (m, 2H), 1.09-1.01 (m, 1H) ppm.

${ }^{13}$ C NMR (101 MHz, Chloroform-d) $\delta=157.4,154.4$ (d, $\left.J=257.2 \mathrm{~Hz}\right), 128.9$ (d, $J=$ $4.3 \mathrm{~Hz}$ ), 127.8 (d, J=5.2 Hz, 2C), 113.6 (2C), 84.2 (d, $J=14.6 \mathrm{~Hz}), 81.6,55.3,37.7$ (d, $J=1.0 \mathrm{~Hz}), 31.5,31.4,31.1,25.2,24.3 \mathrm{ppm}$.

${ }^{19}$ F NMR (376 MHz, Chloroform- $d$ ) $\delta=-90.65$ (s, 1F) ppm.

HRMS (ESI) m/z: [M+H] $]^{+}$Calcd for $\mathrm{C}_{16} \mathrm{H}_{20} \mathrm{FO}_{2} 263.1442$; Found 263.1457

\section{1-mmol-Scale Synthesis for 4aa}

A flask charged with $\mathrm{NaH}(80 \mathrm{mg}, 2.0 \mathrm{mmol}, 2.0$ equiv as a $60 \%$ dispersion in mineral oil) in dry DMF $(6.7 \mathrm{~mL})$ was cooled to $0{ }^{\circ} \mathrm{C}$, before the gem-difluoroalkene 3aa (376 $\mathrm{mg}, 1.0 \mathrm{mmol}, 1.0$ equiv) were added. The reaction was allowed to war up and stir at room temperature for 2 hours, before it was quenched with water. The aqueous phase was extracted with ethyl acetate $(3 \times 50 \mathrm{~mL})$, and the combined organic phases were washed with brine, dried over $\mathrm{Na}_{2} \mathrm{SO}_{4}$, filtered and concentrated in vacuo. The crude 
material was then purified through column chromatography on silica gel (petroleum ether/ethyl acetate $=5: 1$ ) to give the 6-fluoro-3,4-dihydro- $2 \mathrm{H}$-pyran $4 \mathbf{a a}$ as a colorless oil (292 mg, 82\%).

\section{References}

1. Ichitsuka, T.; Fujita, T.; Arita,T.; Ichikawa, J. Double C-F Bond Activation through $\beta$-Fluorine Elimination: Nickel-Mediated [3+2] Cycloaddition of 2-Trifluoromethyl-1alkenes with Alkynes. Angew. Chem. Int. Ed. 2014, 53, 7564-7568.

2. Lan, Y.; Yang, F.; Wang, C. Synthesis of gem-Difluoroalkenes via Nickel-Catalyzed Allylic Defluorinative Reductive Cross-Coupling. ACS Catal. 2018, 8, 9245-9251.

3. Lin, Z.; Lan, Y.; Wang, C. Synthesis of gem-Difluoroalkenes via Nickel-Catalyzed Reductive C-F and C-O Bond Cleavage. ACS Catal. 2019, 9, 775-780.

4. Marcyk, P. T.; Jefferies, L. R.; AbuSalim, D. I.; Maren, P.; Baik, M.-H.; Cook, S. P. Stereoinversion of Unactivated Alcohols by Tethered Sulfonamides. Angew. Chem. Int. Ed. 2019, 58, $1727-1731$.

5. Huang, J.; Du, D.-M.; Xu, J. Facile Synthesis of 1,1-Disubstituted Taurines. Synthesis, 2006, 2006, 315-319.

6. Ellis, G. L.; Amewu, R.; Sabbani, S.; Stocks, P. A.; Shone, A.; Stanford, D.; Gibbons, P.; Davies, J.; Vivas, L.; Charnaud, S.; Bongard, E.; Hall, C.; Rimmer, K.; Lozanom, S.; Jesús, M.; Gargallo, D.; Ward, S. A.; O’Neill, P. M. Two-Step Synthesis of Achiral Dispiro-1,2,4,5-tetraoxanes with Outstanding Antimalarial Activity, Low Toxicity, and High-Stability Profiles. J. Med. Chem. 2008, 51, 2170-2177.

7. ElMarrouni, A.; Lebeuf, R.; Gebauer, J.; Heras, M.; Arseniyadis, S.; Cossy, J. Total Synthesis of Nominal Lyngbouilloside Aglycon. Org. Lett. 2012, 14, 314-317.

8. Muehlbach, M.; Poulter, C. D. Regioselective Opening of Simple Epoxides with Diisopropylamine Trihydro fluoride. J. Org. Chem. 1988, 53, 1026-1030.

9. Imoto, S.; Kohgo, S.; Tokuda, R.; Kumamoto, H.; Aoki, M.; Amano, M.; KuwataHigashi, N.; Mitsuya, H.; Haragushi, K. Design, Synthesis, and Evaluation of AntiHBV Activity of Hybrid Molecules of Entecavir and Adefovir: Exomethylene Acycloguanine Nucleosides and Their Monophosphate Derivatives. Nucleosides, Nucleotides and Nucleic Acids, 2015, 34, 590-602.

10. Nasrallah, A.; Grelier, G.;Lapuh, M. I.; Duran, F. J.; Darses, B.; Dauban, P. Dirhodium(II)-Mediated Alkene Epoxidation with Iodine(III) Oxidants. Eur. J. Org. Chem. 2018, 2018, 5836-5842.

11. Li, S.; Chen, X.; Xu, J. Microwave-Assisted Copper-Catalyzed Stereoselective Ring Expansion of Three-Membered Heterocycles with $\alpha$-Diazo- $\beta$-Dicarbonyl Compounds. Tetrahedron, 2018, 74, 1613-1620. 


\section{NMR Spectra}

${ }^{1} \mathrm{H}$ NMR-spectrum $\left(400 \mathrm{MHz}, \mathrm{CDCl}_{3}\right.$ ) of $\mathbf{2 h}$

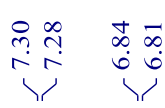

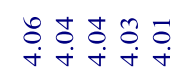

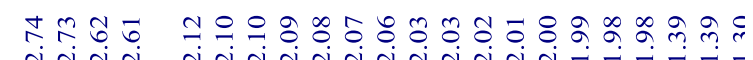

Y Y
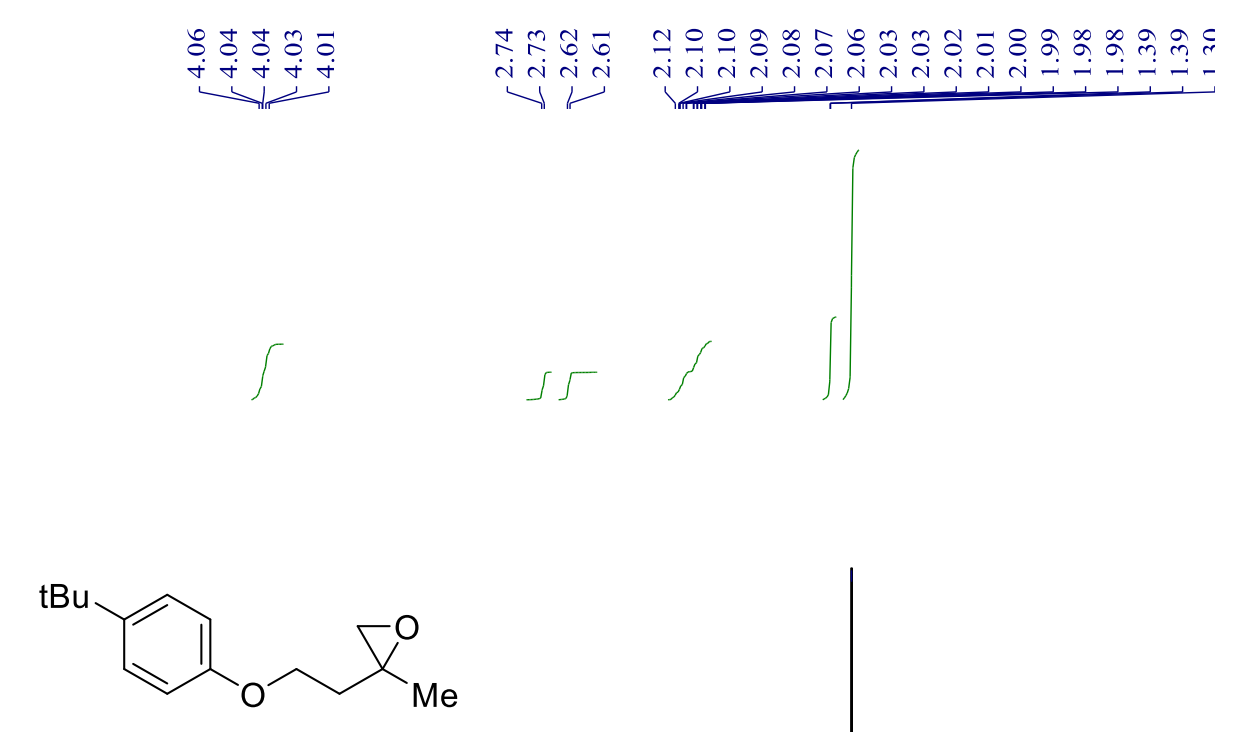

$2 h$

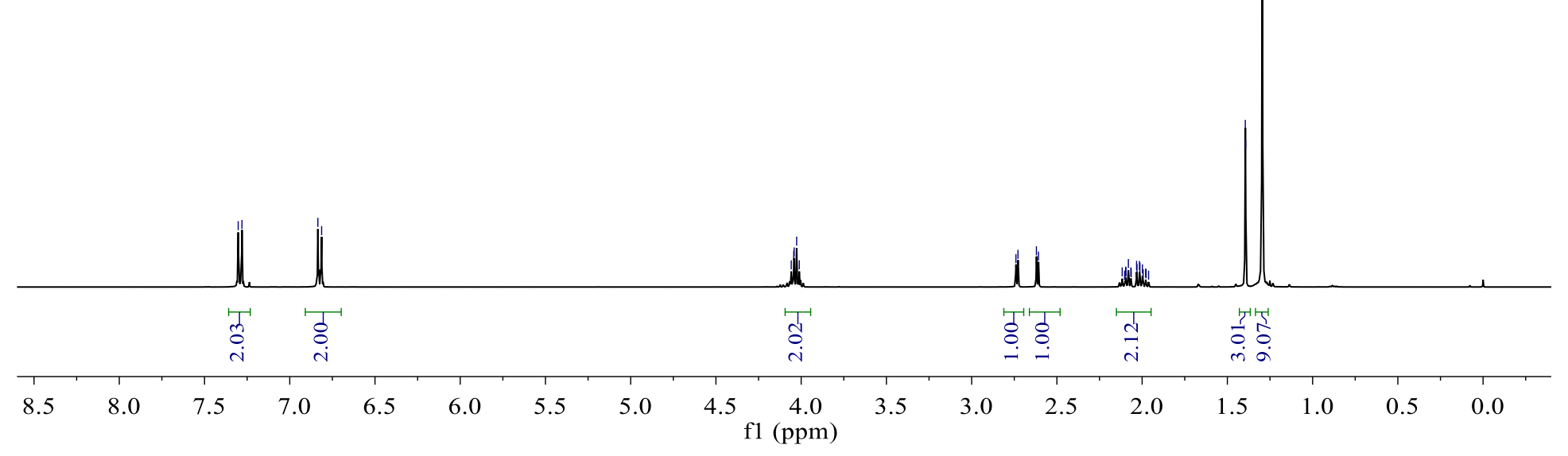

\$32 
${ }^{13} \mathrm{C} \mathrm{NMR}$-spectrum $\left(101 \mathrm{MHz}, \mathrm{CDCl}_{3}\right)$ of $\mathbf{2 h}$

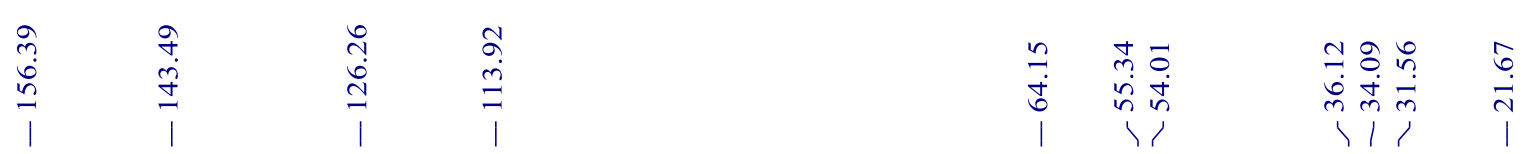

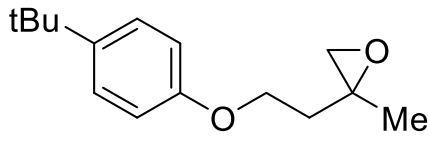

$2 h$
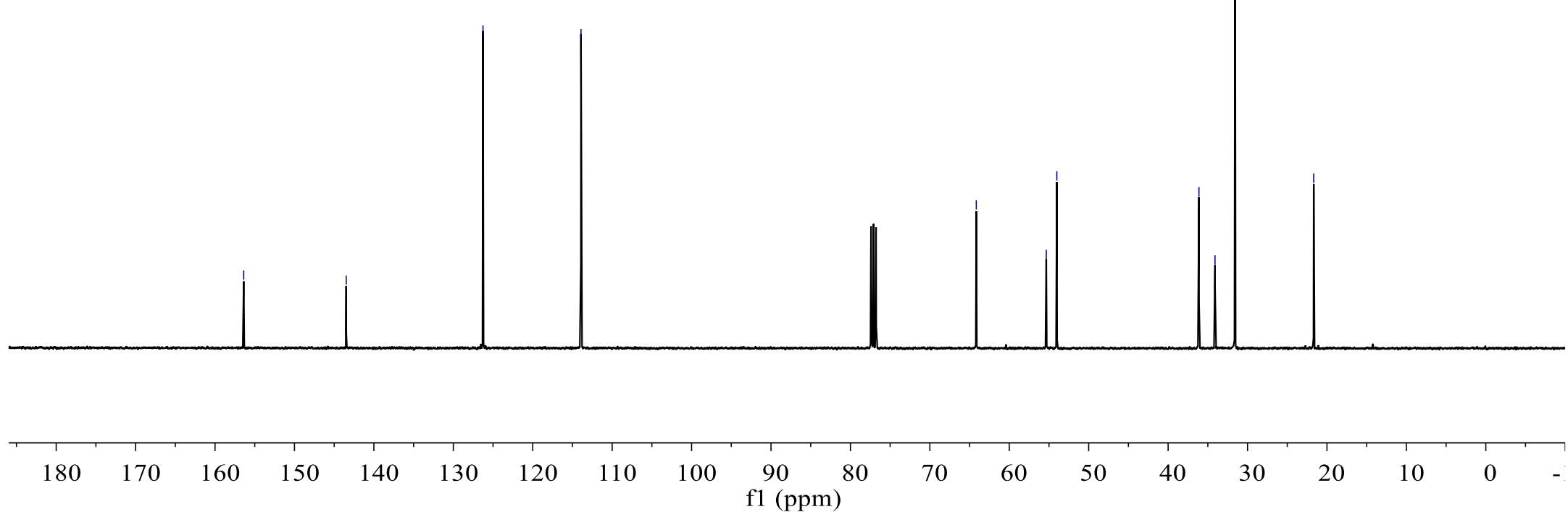

S33 
${ }^{1} \mathrm{H}$ NMR-spectrum (400 MHz, $\mathrm{CDCl}_{3}$ ) of $\mathbf{2} \mathbf{j}$

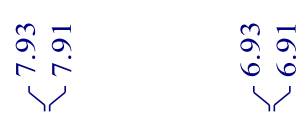

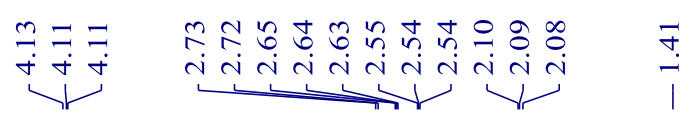
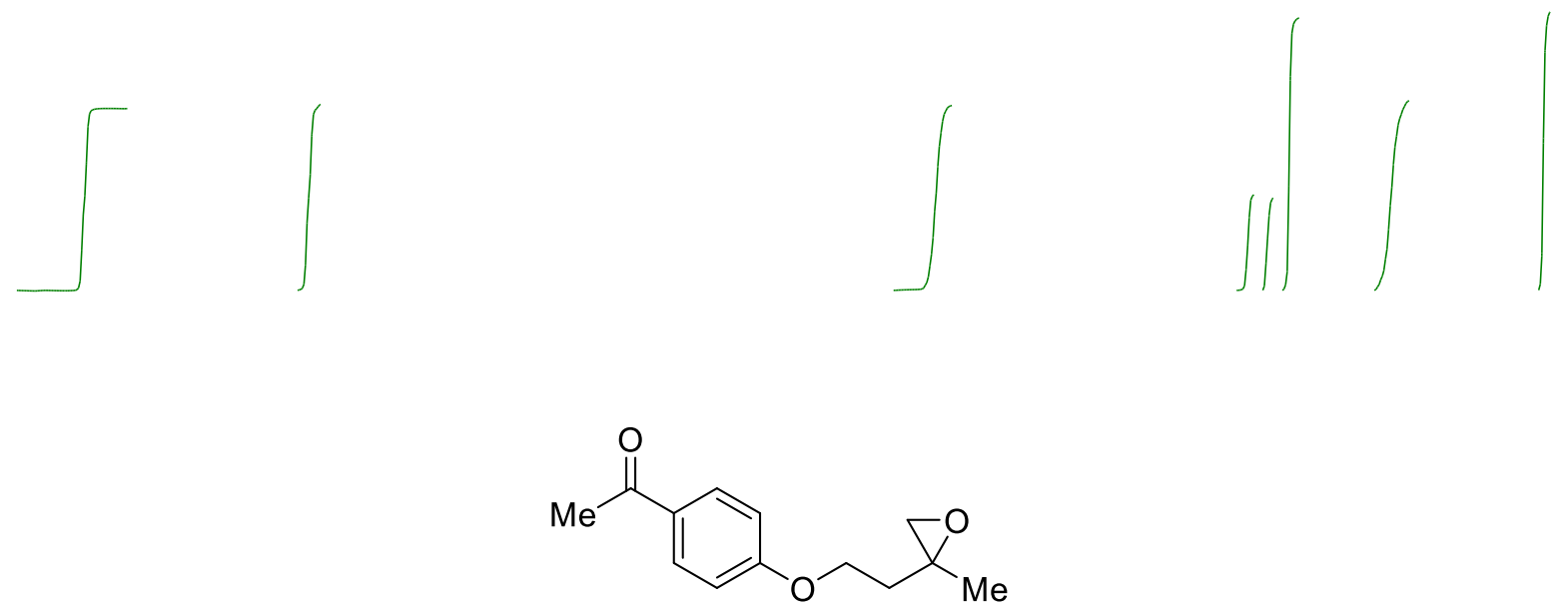

2j

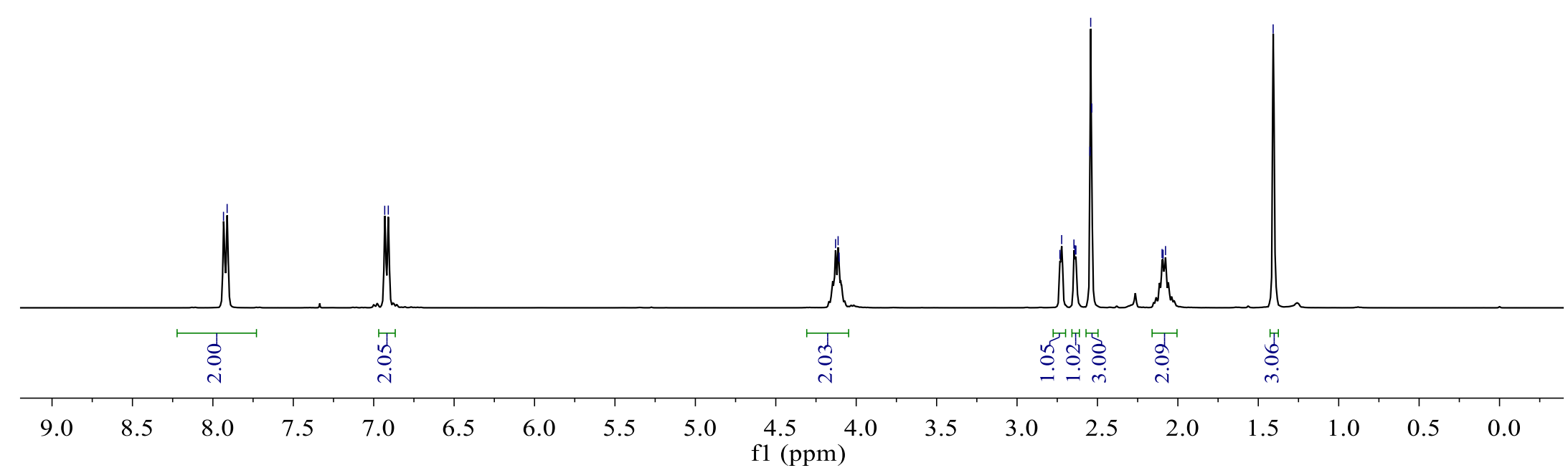

S34 
${ }^{13} \mathrm{C}$ NMR-spectrum (101 MHz, $\left.\mathrm{CDCl}_{3}\right)$ of $\mathbf{2} \mathbf{j}$

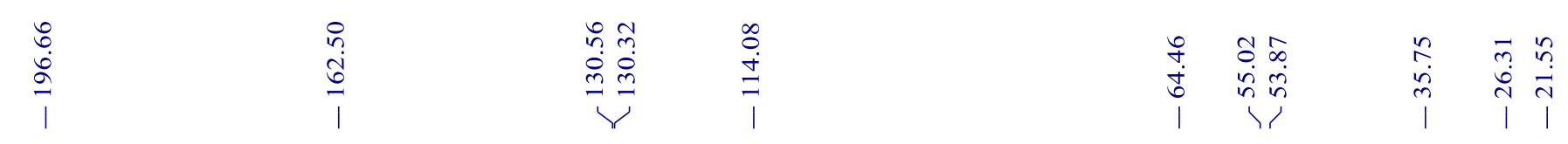

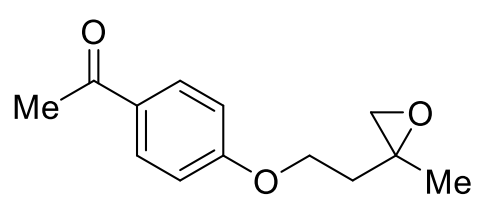

2j

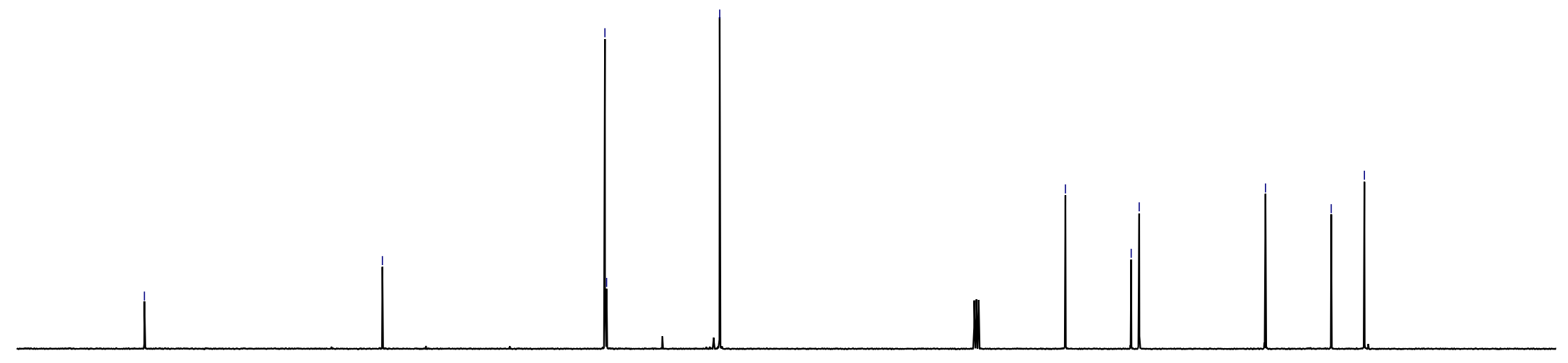

$\begin{array}{llllllllllll}210 & 200 & 190 & 180 & 170 & 160 & 150 & 140 & 130 & 120 & 110 & 100\end{array}$ f1 (ppm)

S35 
${ }^{1} \mathrm{H}$ NMR-spectrum $\left(400 \mathrm{MHz}, \mathrm{CDCl}_{3}\right)$ of $\mathbf{2 n}$

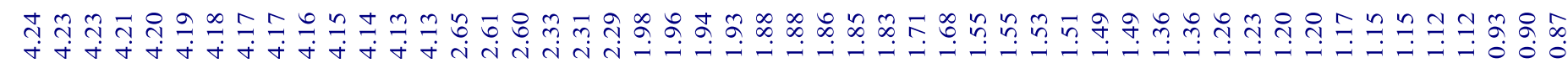
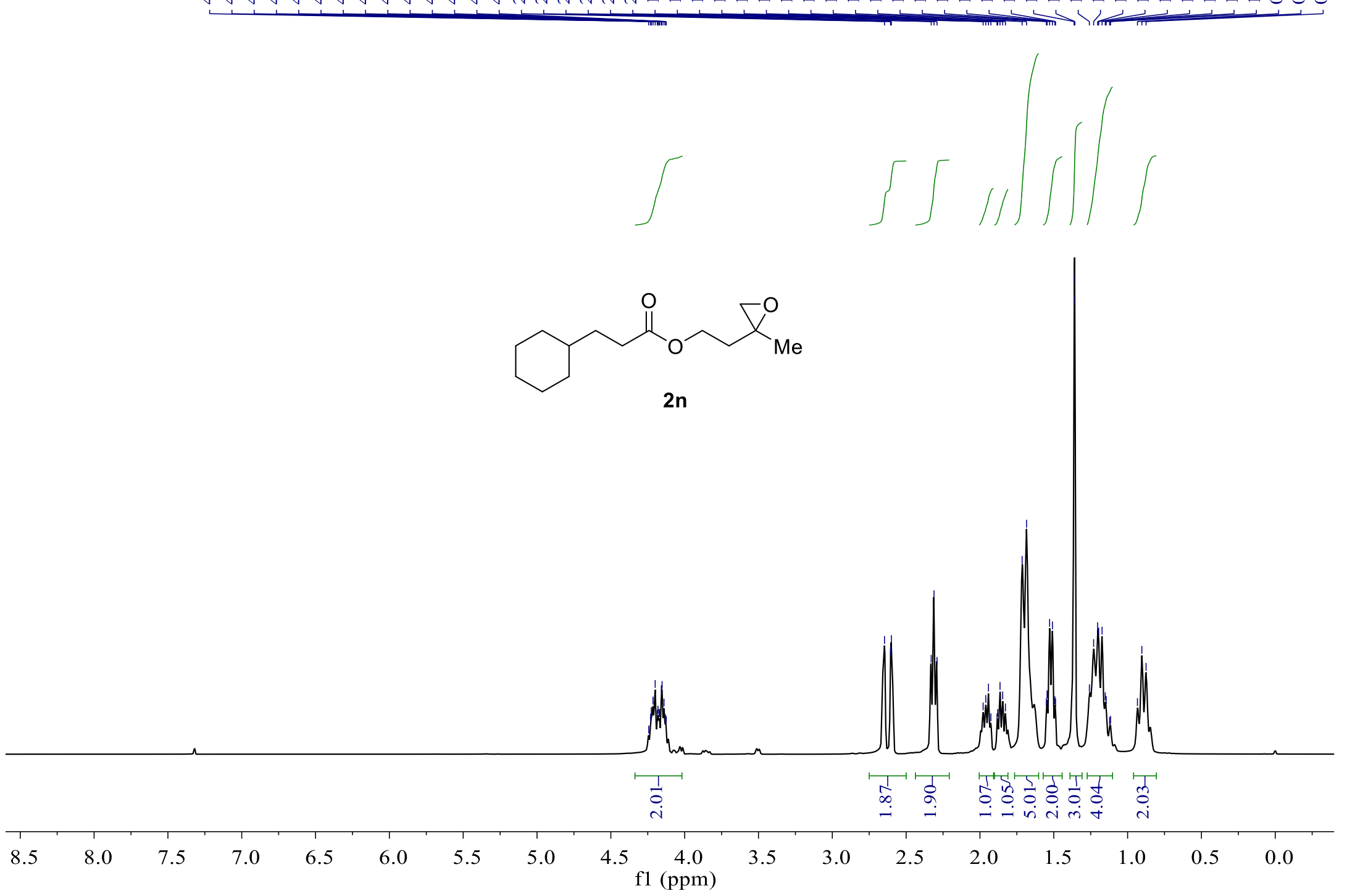

S36 
${ }^{13} \mathrm{C}$ NMR-spectrum $\left(101 \mathrm{MHz}, \mathrm{CDCl}_{3}\right)$ of $\mathbf{2 n}$
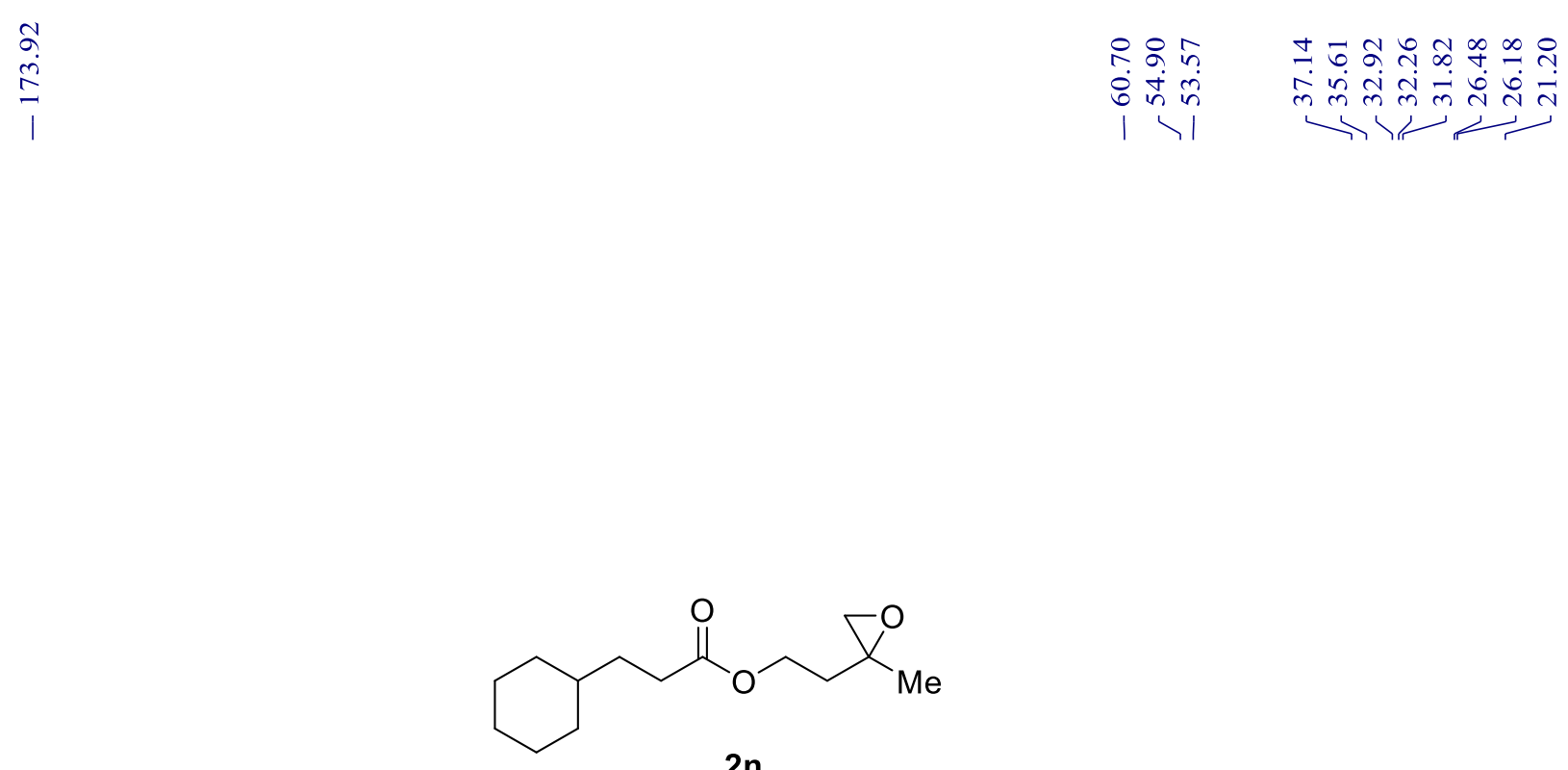

2n

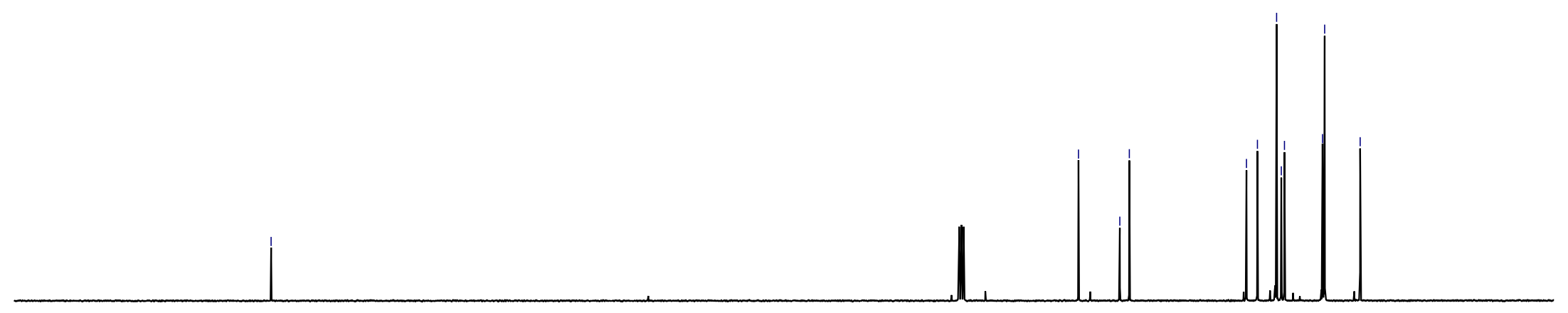

$10 \quad 200 \quad 190$

$80 \quad 1$

16

150

$140 \quad 130$

$0 \quad 120$

$110 \quad 100$

f1 (ppm)

$\begin{array}{lll}80 & 70 & 60\end{array}$

$50 \quad 40$

S37 
${ }^{1} \mathrm{H}$ NMR-spectrum $\left(400 \mathrm{MHz}, \mathrm{CDCl}_{3}\right)$ of $\mathbf{2 o}$

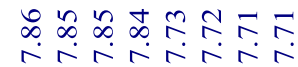

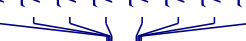

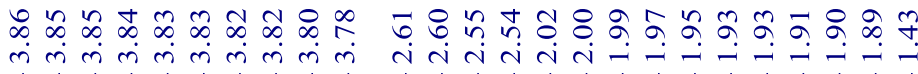
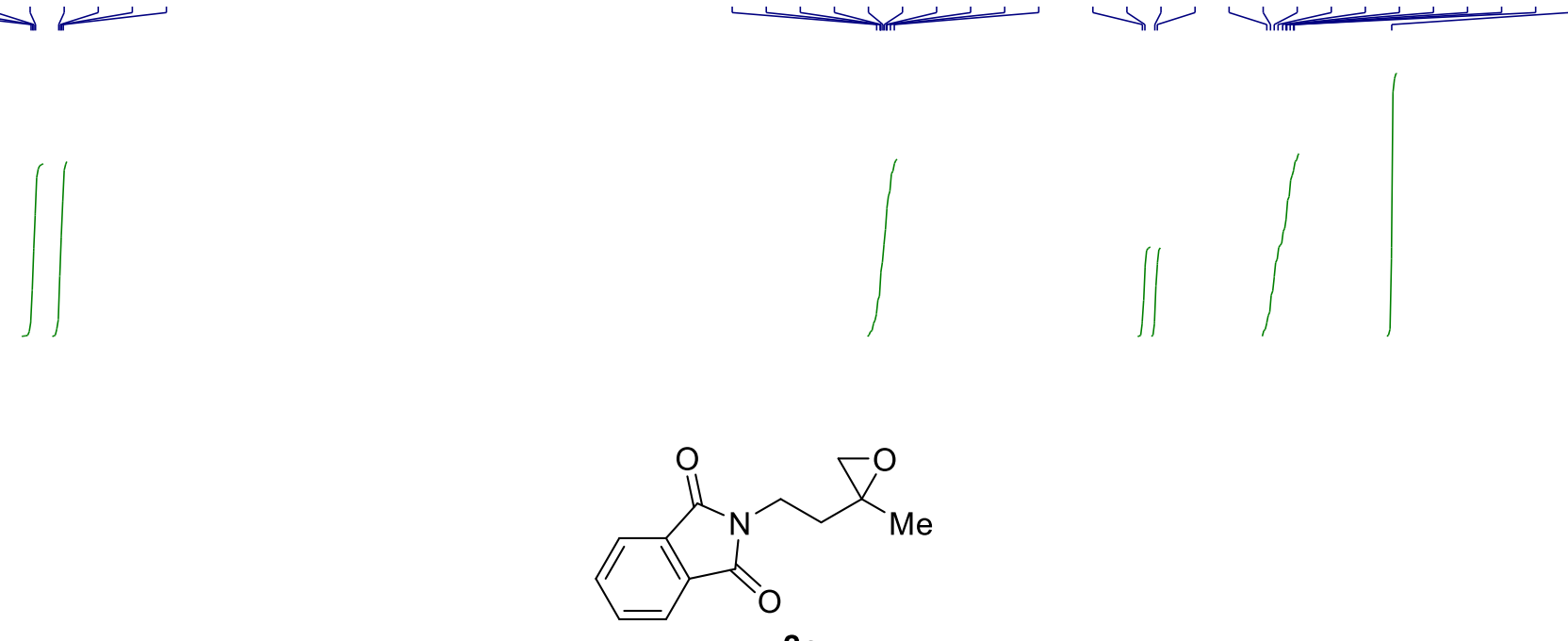

20

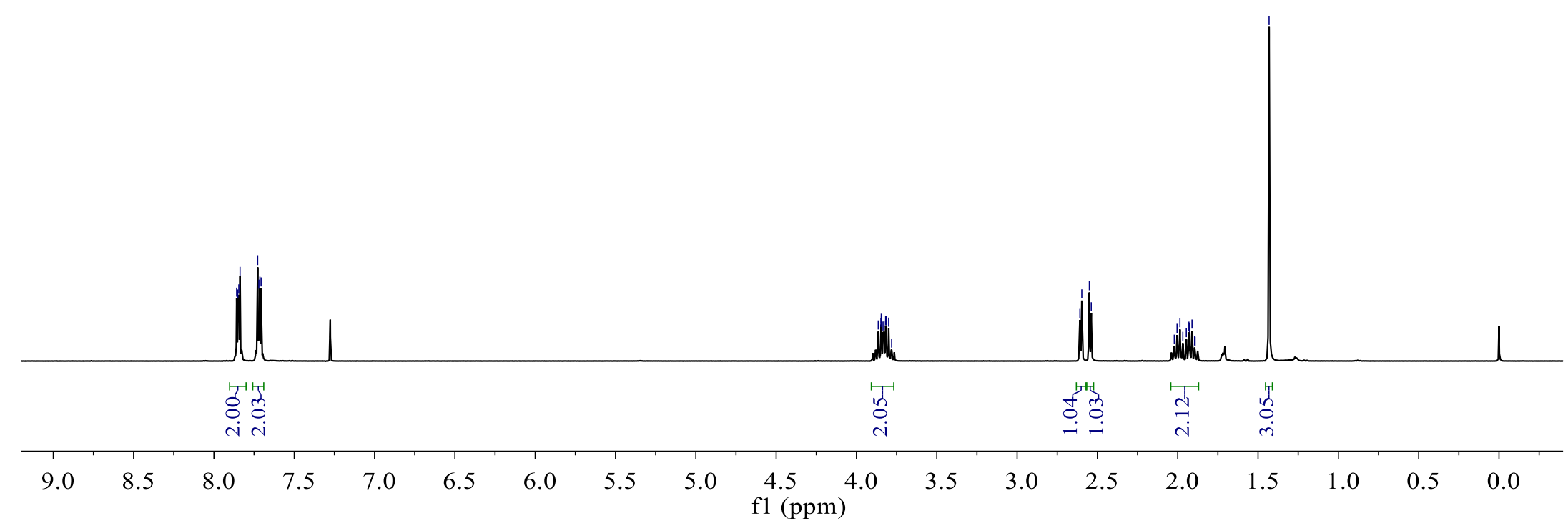

S38 
${ }^{13} \mathrm{C}$ NMR-spectrum $\left(101 \mathrm{MHz}, \mathrm{CDCl}_{3}\right)$ of $\mathbf{2 o}$

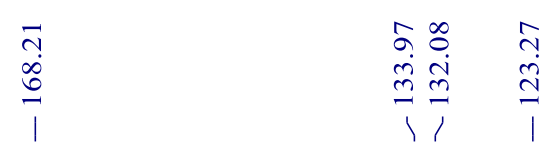

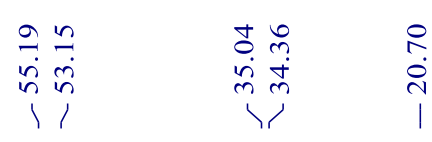

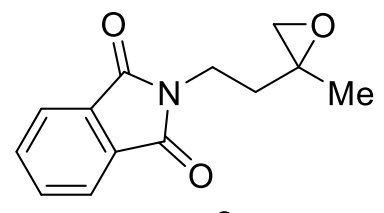

20
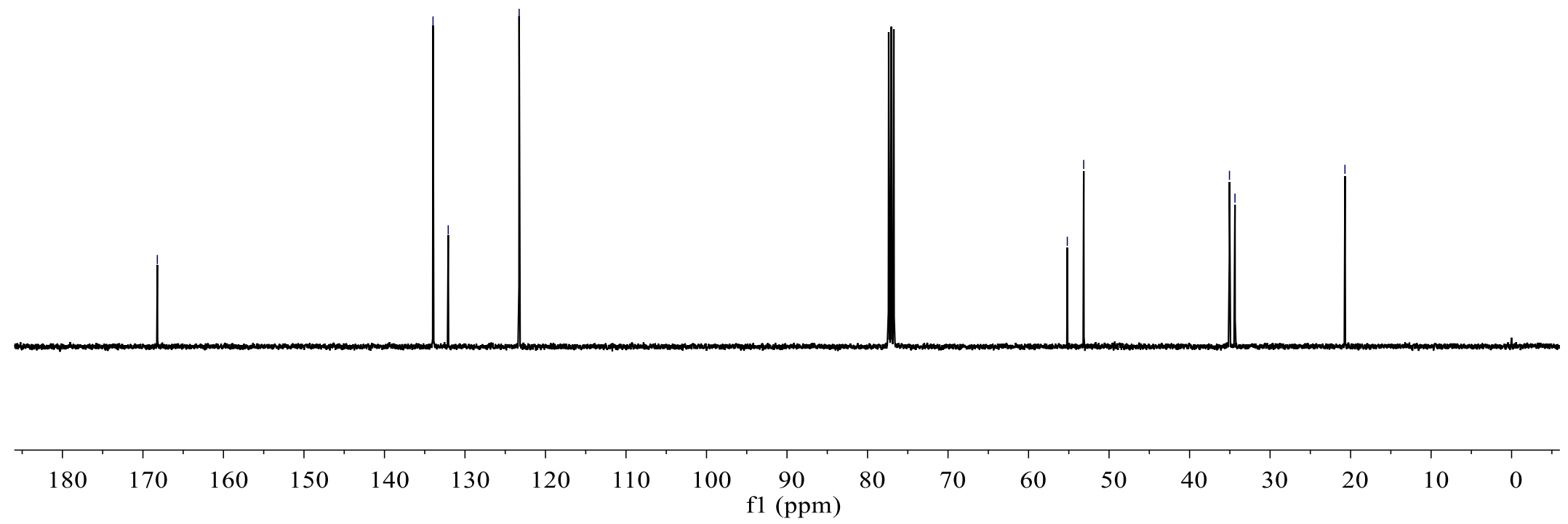

S39 
${ }^{1} \mathrm{H}$ NMR-spectrum $\left(500 \mathrm{MHz}, \mathrm{CDCl}_{3}\right.$ ) of $\mathbf{S - 1}$

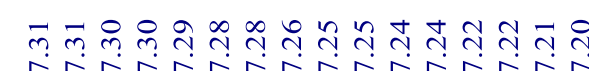

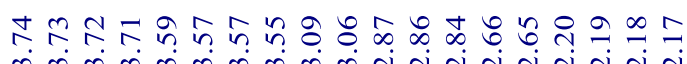

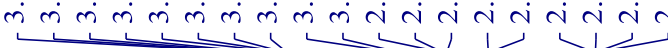
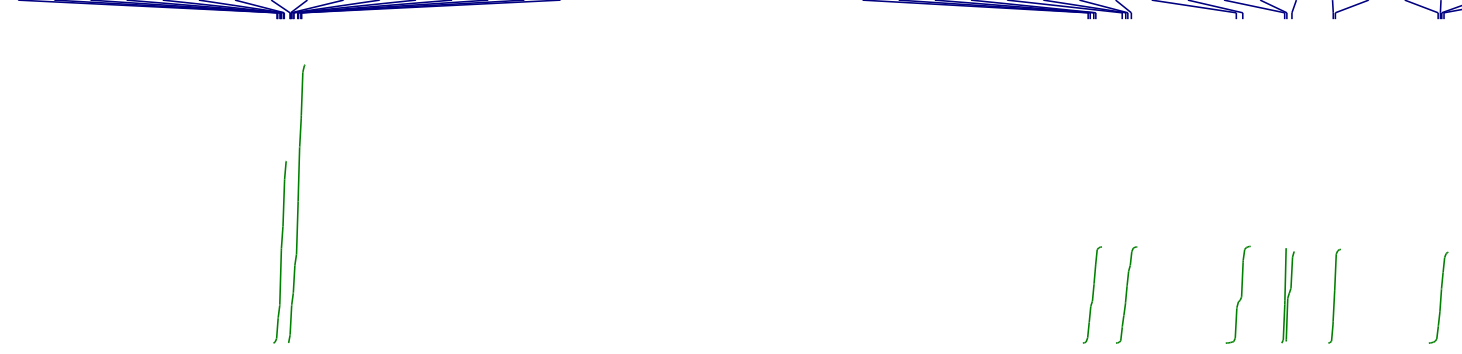

$1>\overbrace{\mathrm{O}} \mathrm{OH}$

S-1

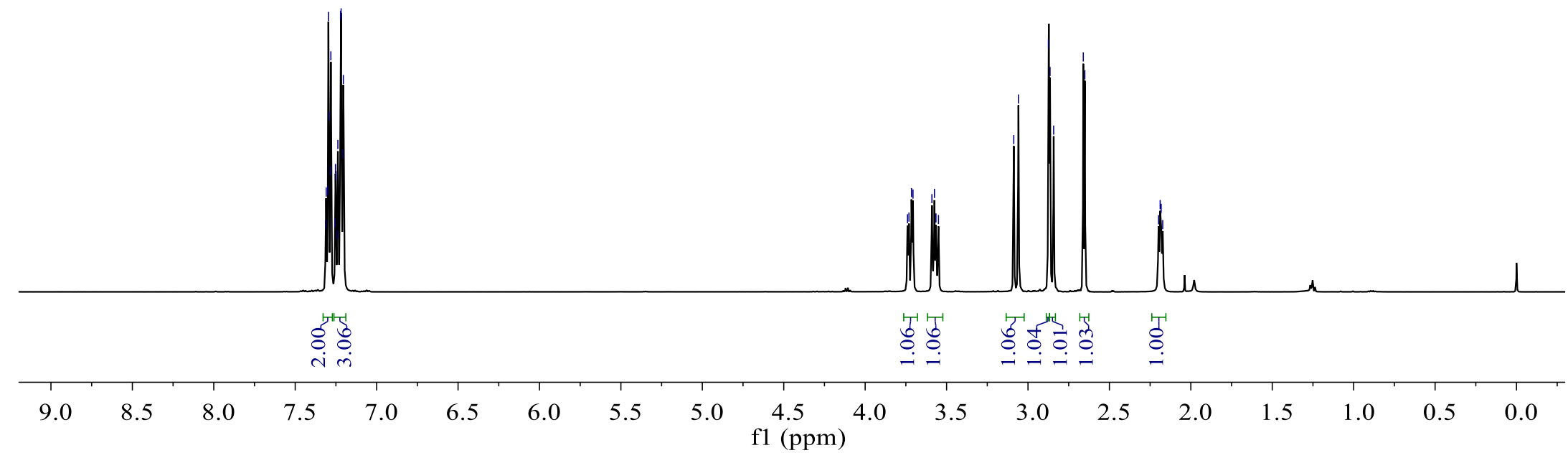

540 
${ }^{13} \mathrm{C}$ NMR-spectrum $\left(126 \mathrm{MHz}, \mathrm{CDCl}_{3}\right)$ of $\mathbf{S - 1}$

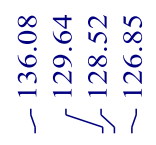

$\begin{array}{cccc}\vec{\infty} & 0 & 0 & n \\ i & 0 & 0 \\ 0 & 0 & \infty \\ 1 & 1 & 1 & 1\end{array}$
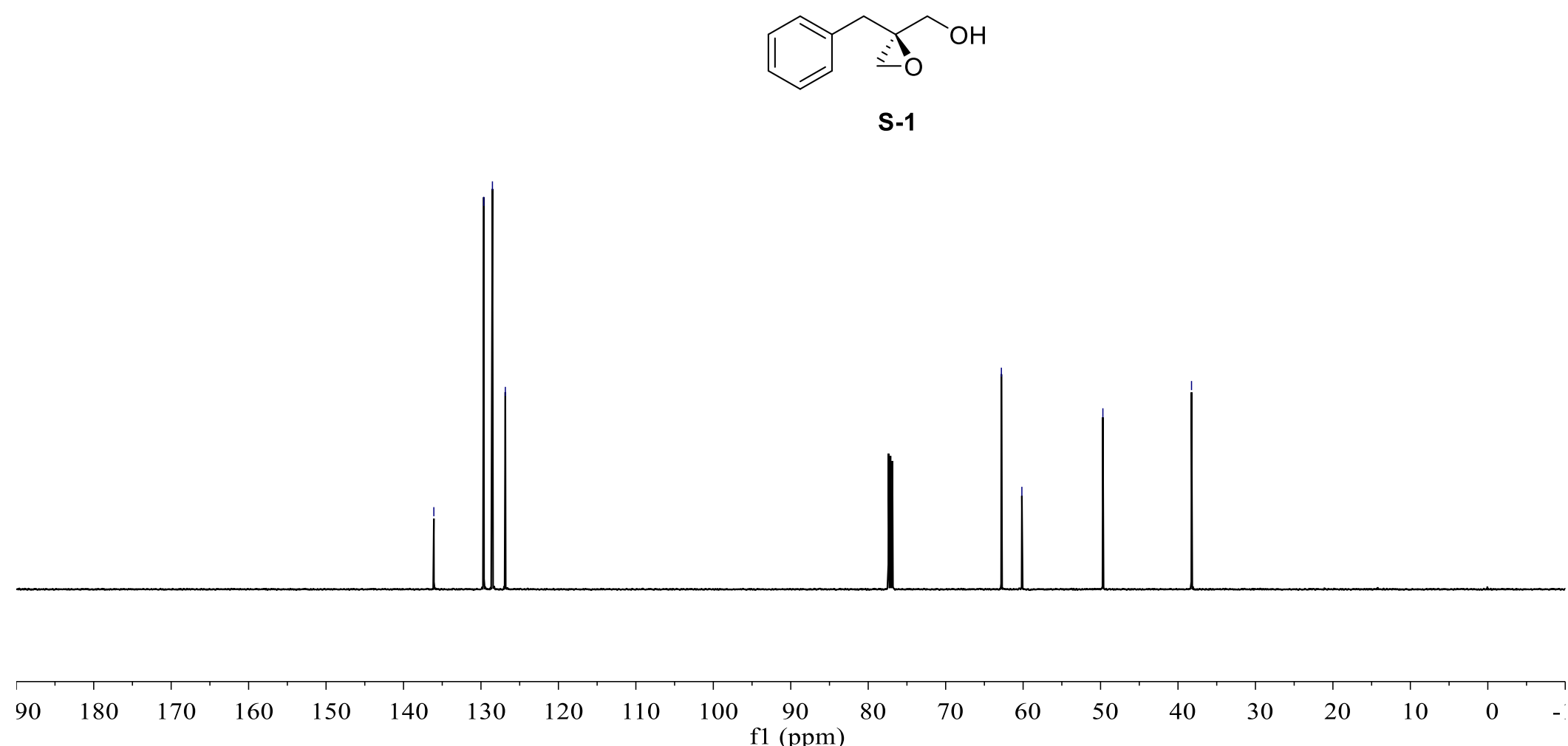

S41 
${ }^{1} \mathrm{H}$ NMR-spectrum $\left(500 \mathrm{MHz}, \mathrm{CDCl}_{3}\right)$ of $\mathbf{2 g}$

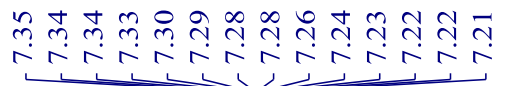

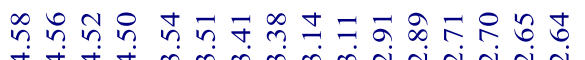

L

$\sqrt{r+a_{n}}$
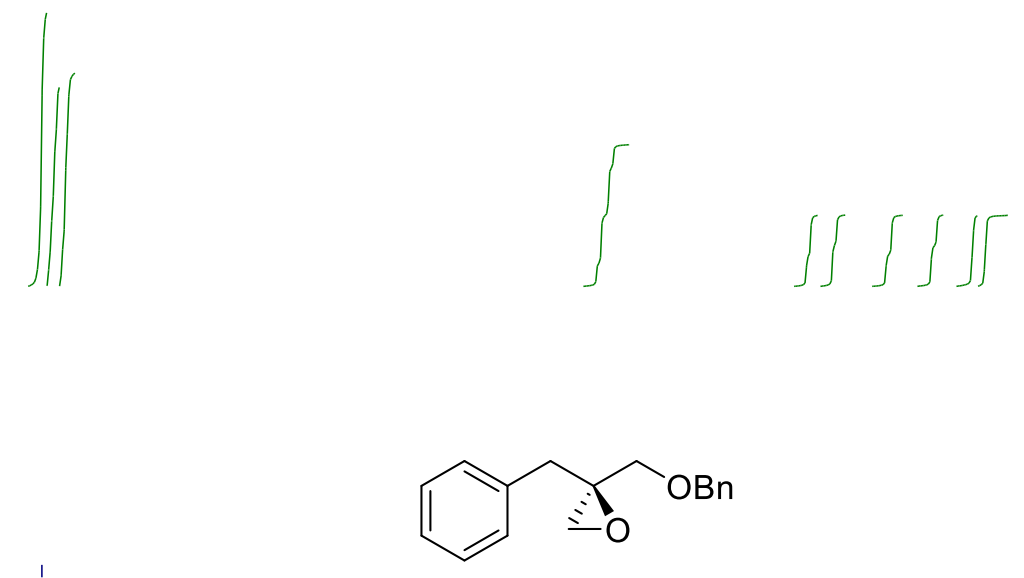

2g

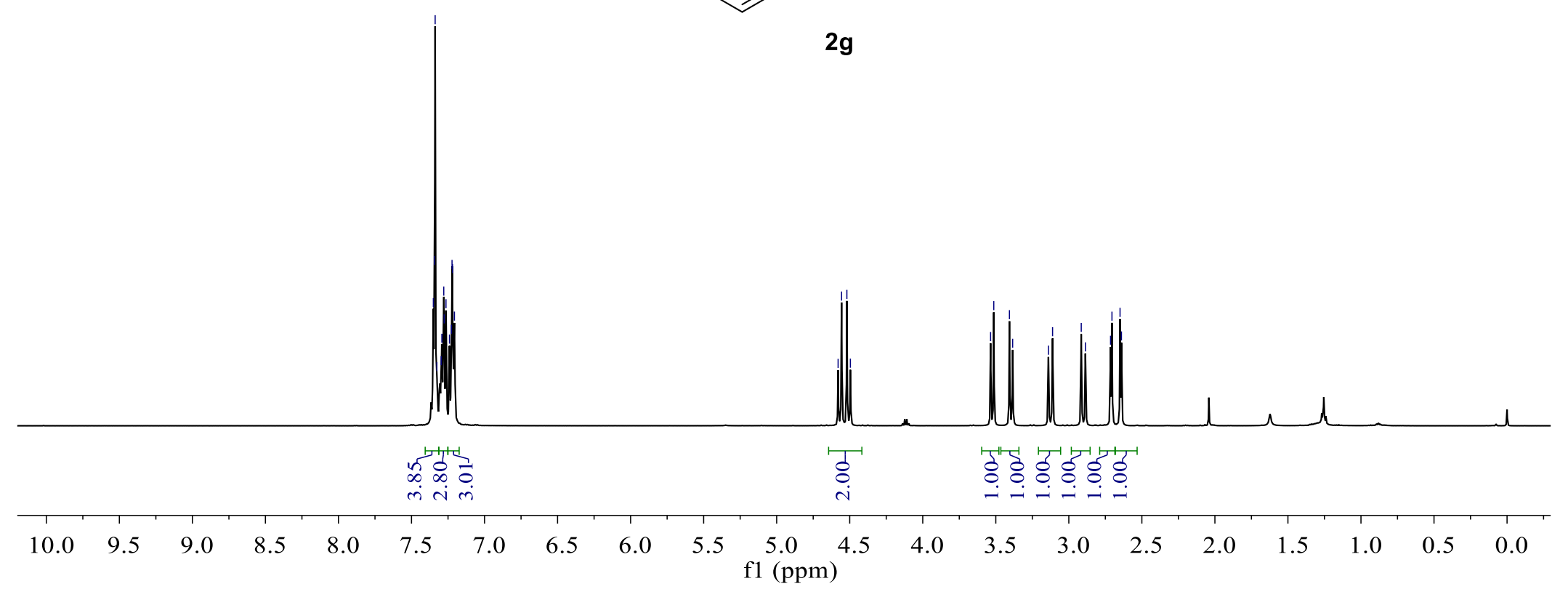

S42 
${ }^{13} \mathrm{C}$ NMR-spectrum $\left(126 \mathrm{MHz}, \mathrm{CDCl}_{3}\right)$ of $\mathbf{2 g}$

$$
\begin{aligned}
& \text { a }
\end{aligned}
$$

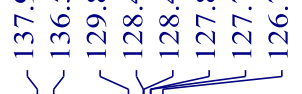

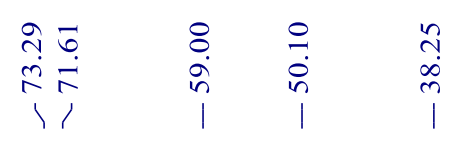

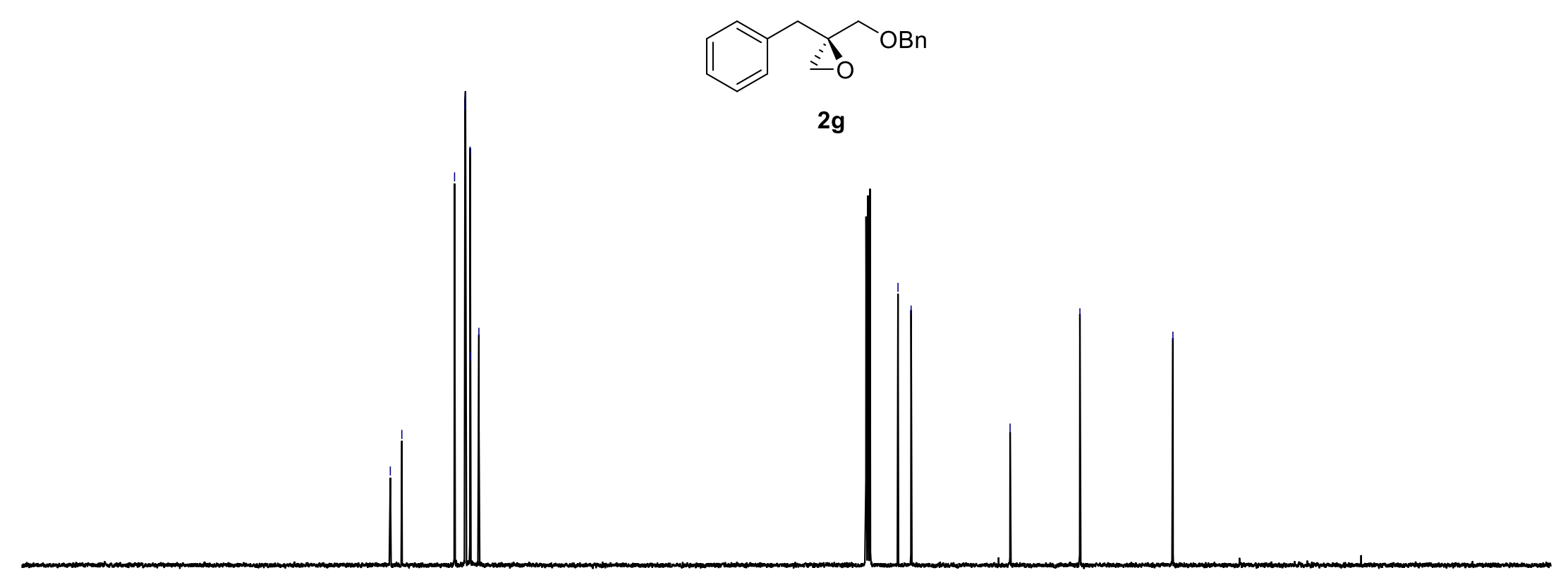

$180 \quad 170$ 160 150 140 130 fl (ppm)

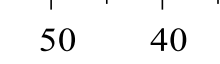

s43 
${ }^{1} \mathrm{H}$ NMR-spectrum (400 MHz, $\mathrm{CDCl}_{3}$ ) of $\mathbf{3 a a}$

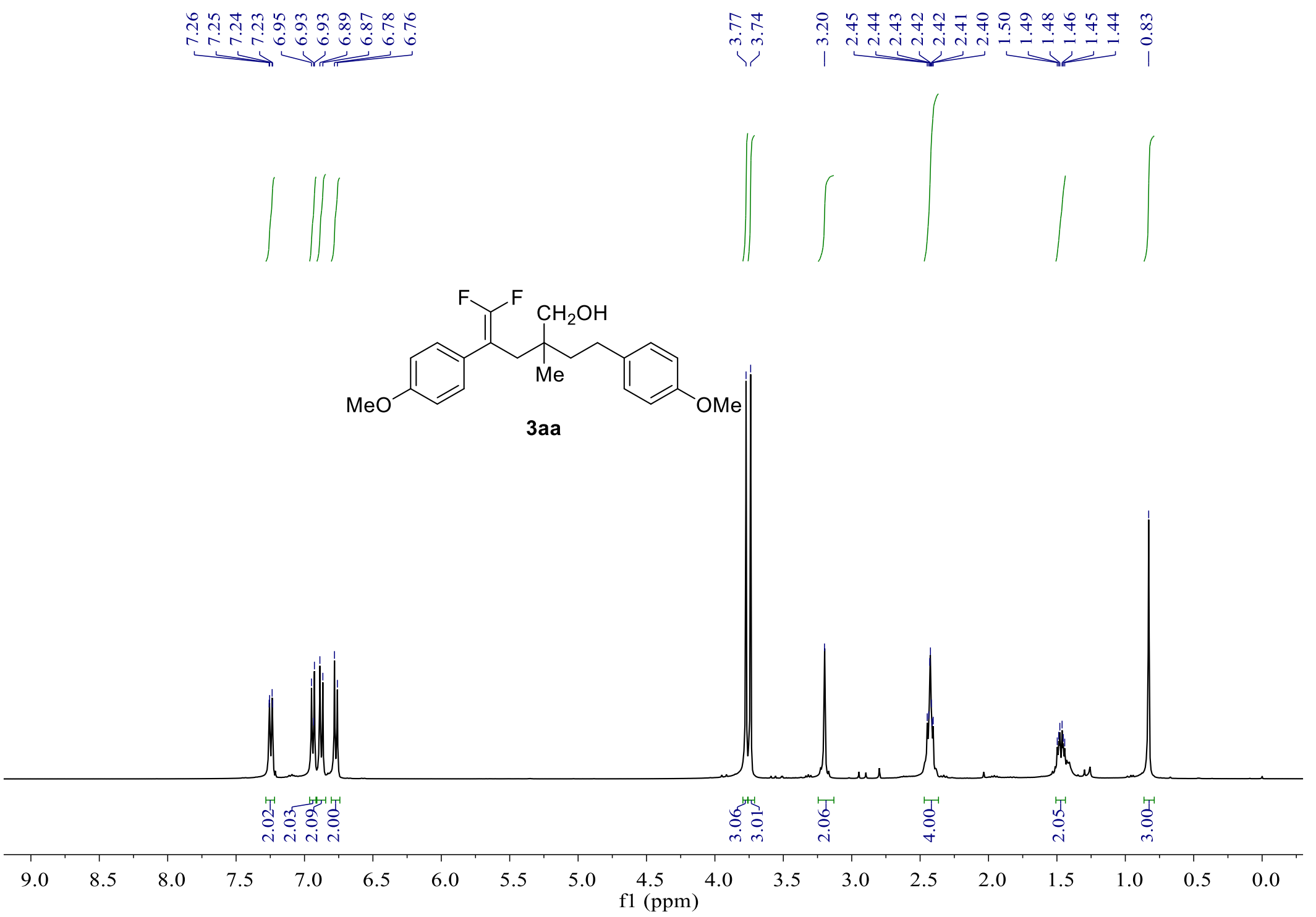

S44 

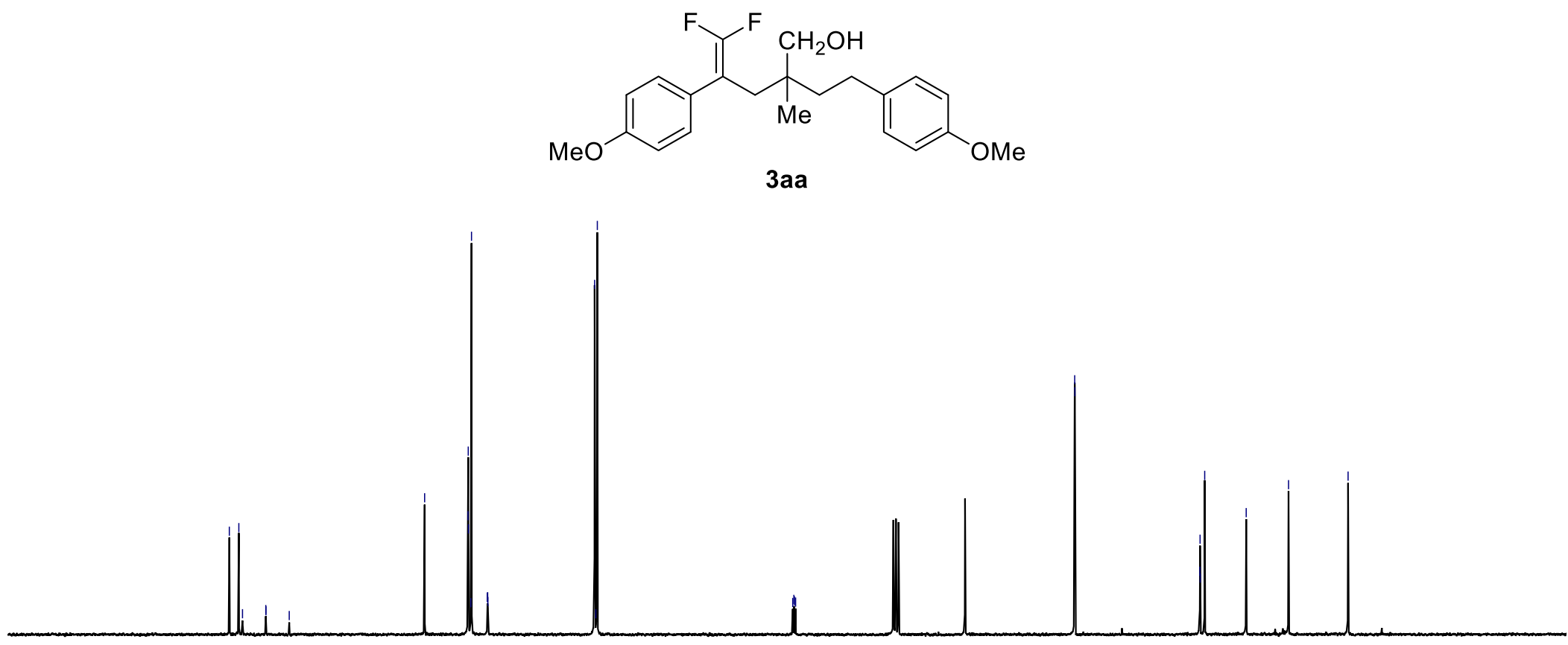
${ }^{19} \mathrm{~F}$ NMR-spectrum $\left(376 \mathrm{MHz}, \mathrm{CDCl}_{3}\right.$ ) of 3aa

$$
\left.\right|_{||} ^{\mid}
$$
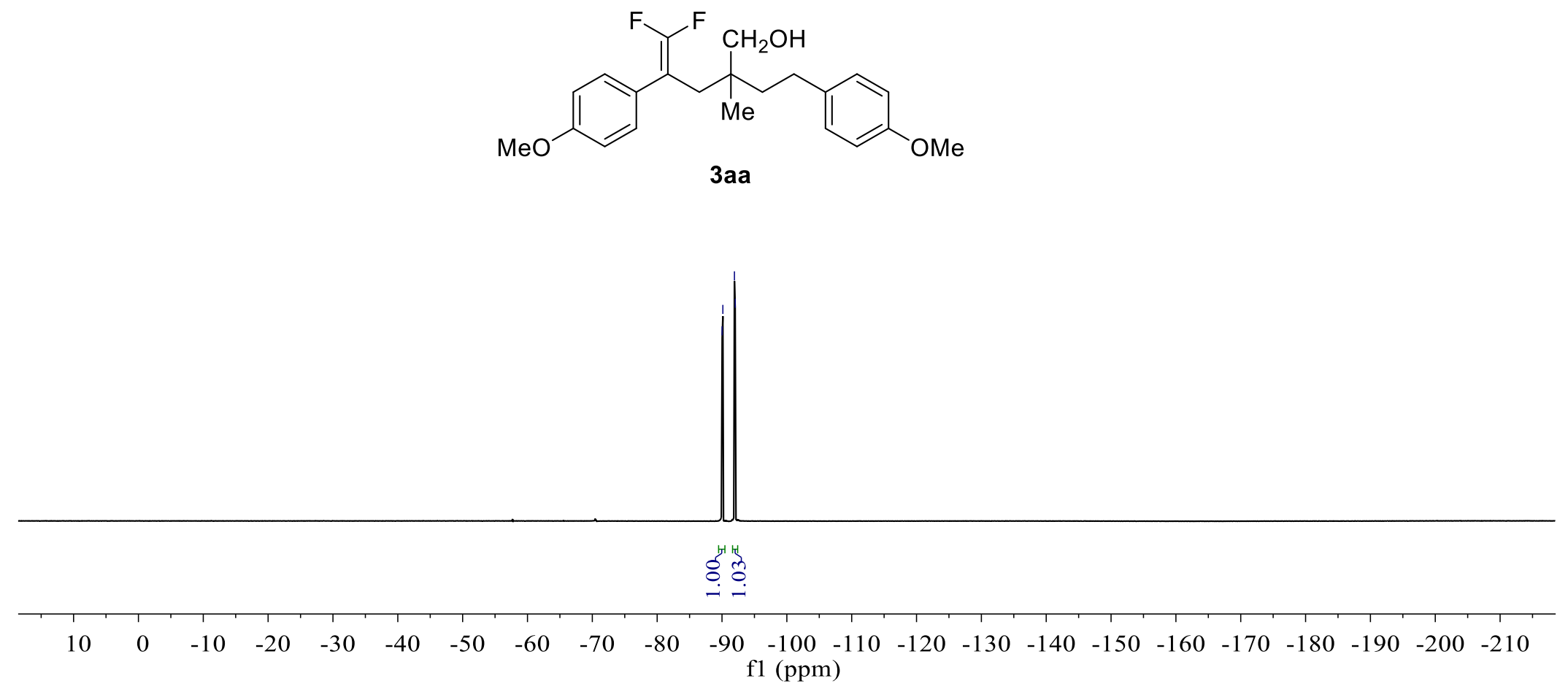
${ }^{1} \mathrm{H}$ NMR-spectrum $\left(400 \mathrm{MHz}, \mathrm{CDCl}_{3}\right)$ of $\mathbf{3 b a}$

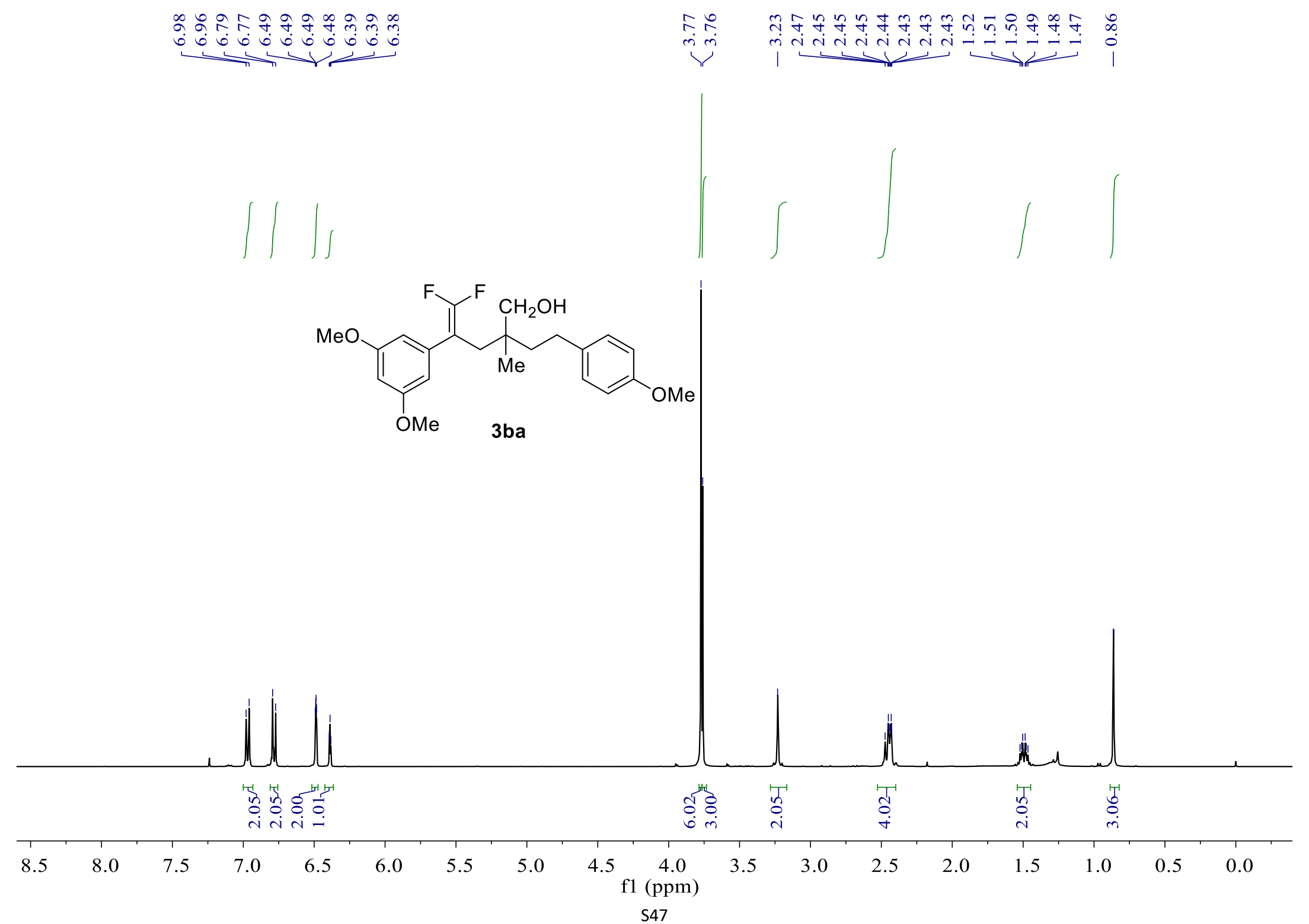


${ }^{13} \mathrm{C}$ NMR-spectrum $\left(101 \mathrm{MHz}, \mathrm{CDCl}_{3}\right)$ of $\mathbf{3 b a}$
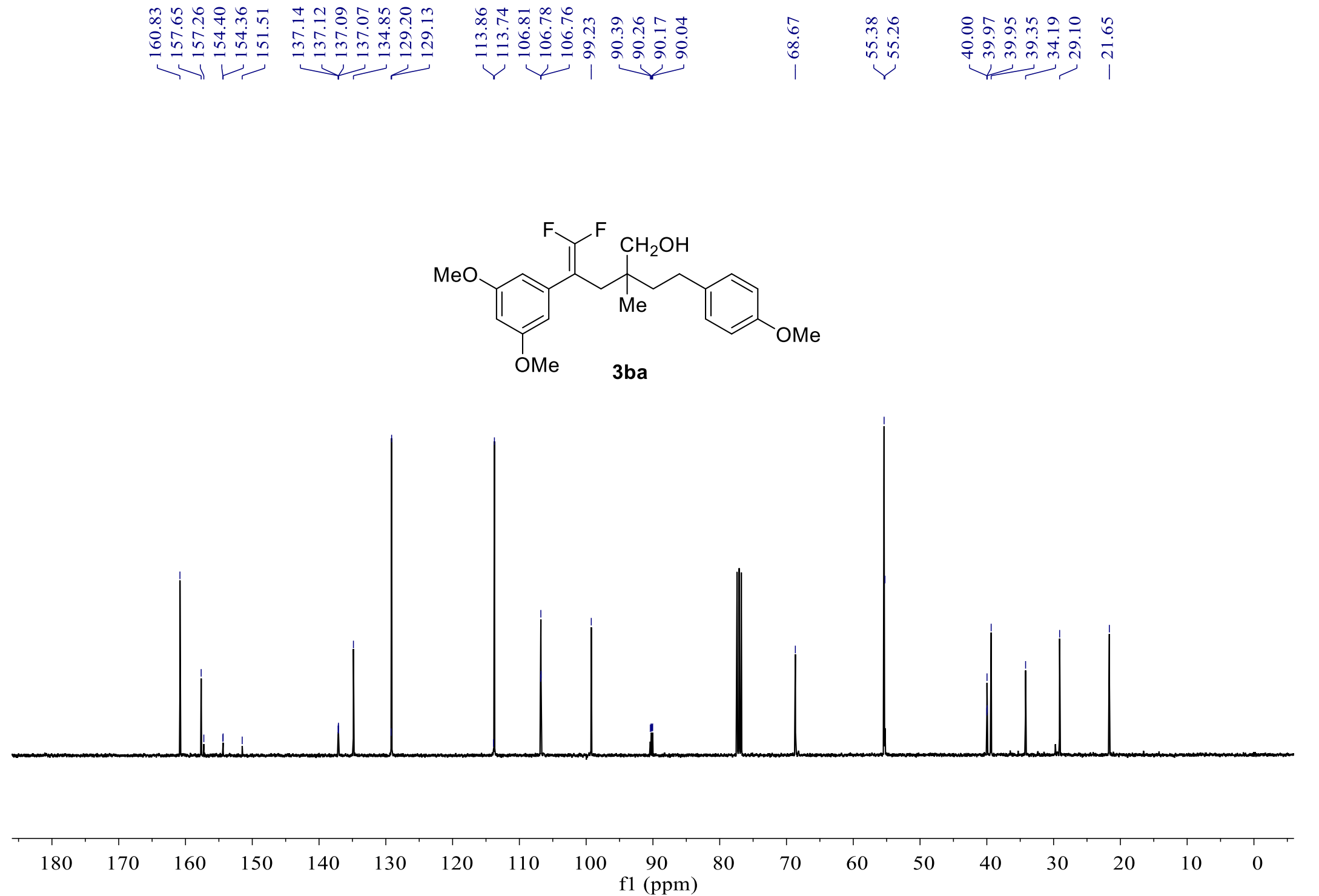
${ }^{19} \mathrm{~F}$ NMR-spectrum (376 MHz, $\mathrm{CDCl}_{3}$ ) of $\mathbf{3 b a}$

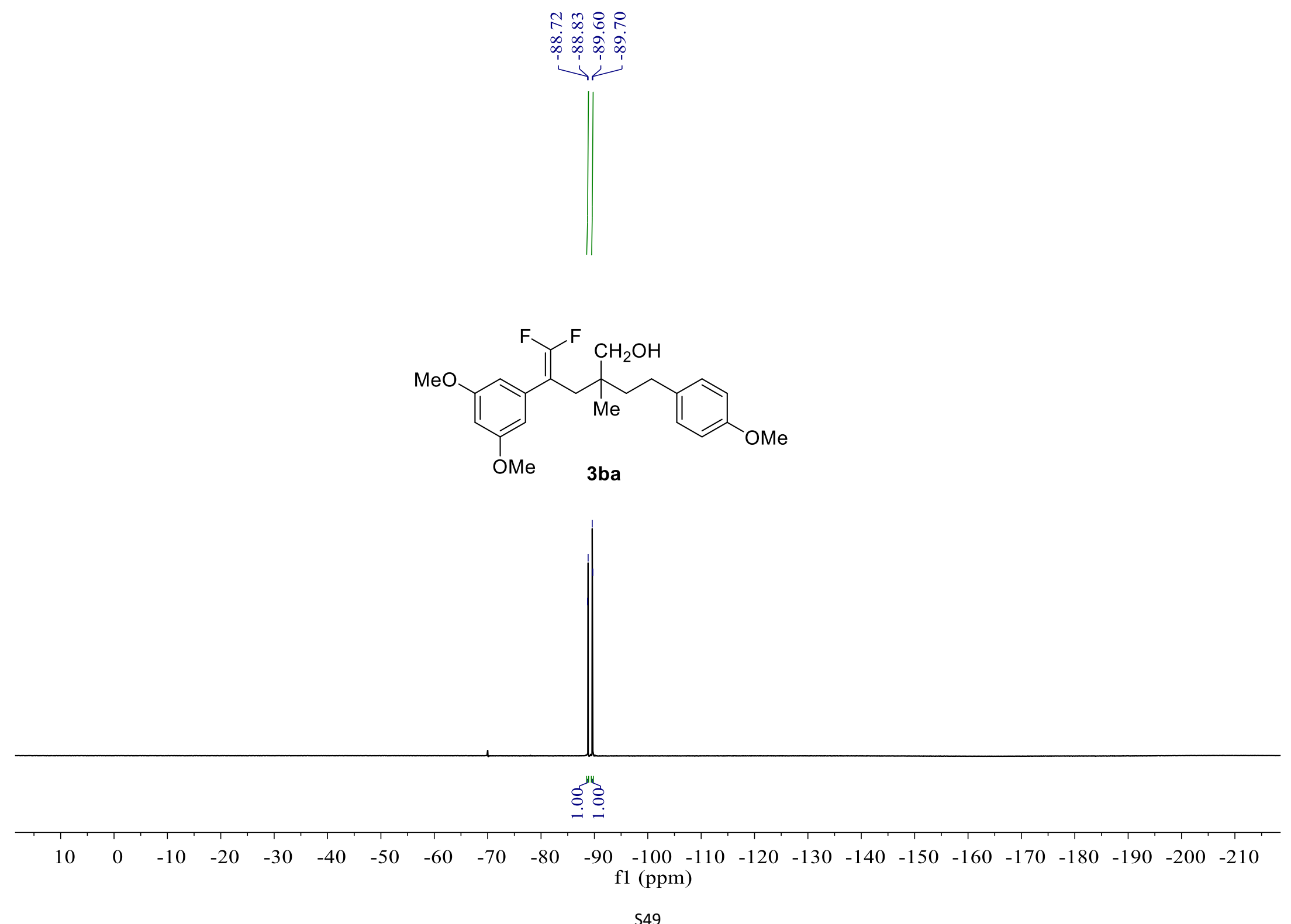

549 
m.

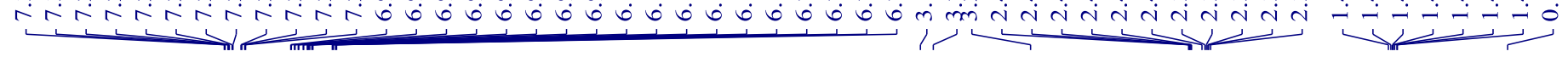
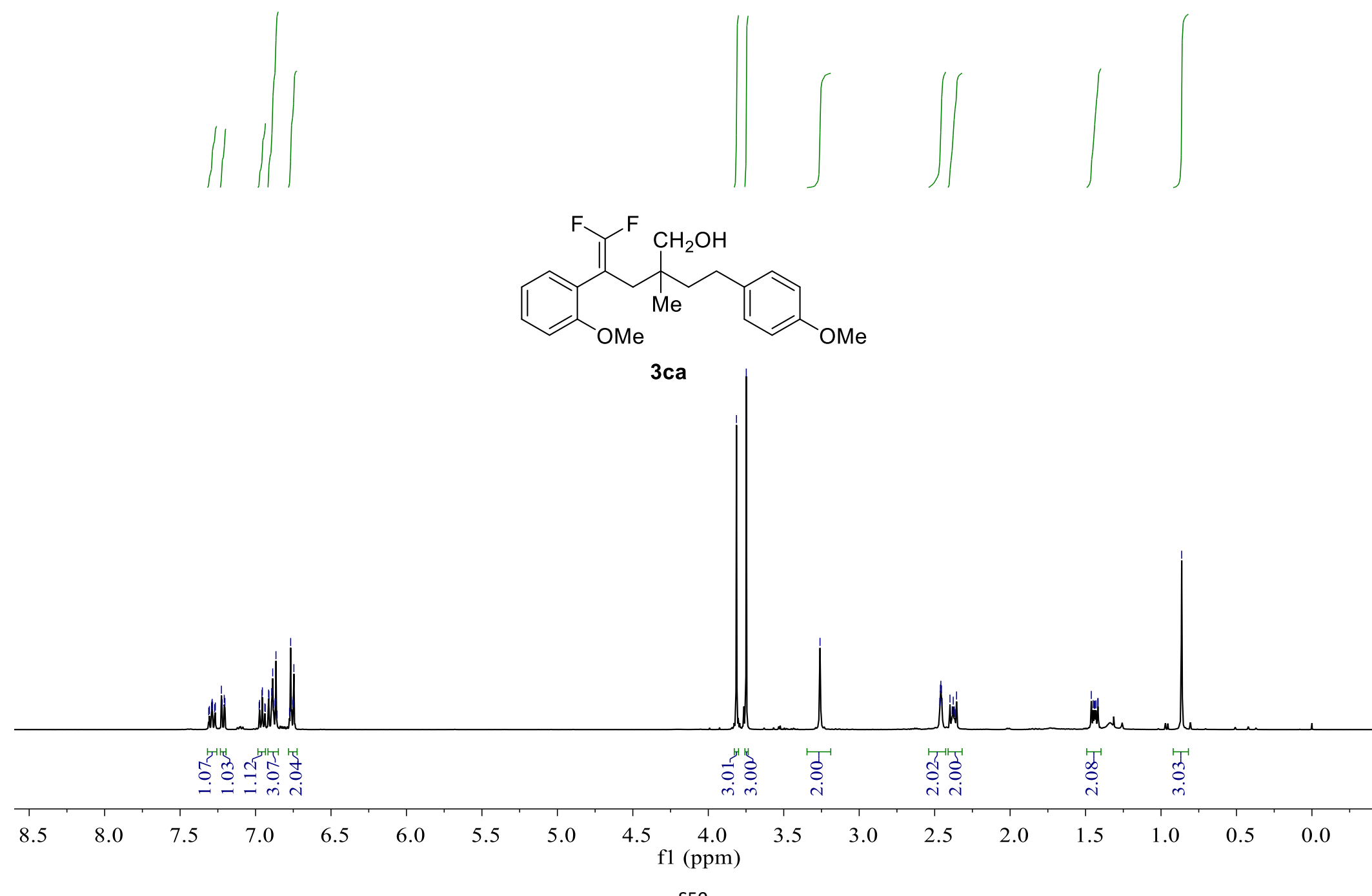

550 
${ }^{13} \mathrm{C}$ NMR-spectrum $\left(101 \mathrm{MHz}, \mathrm{CDCl}_{3}\right)$ of $\mathbf{3 c a}$

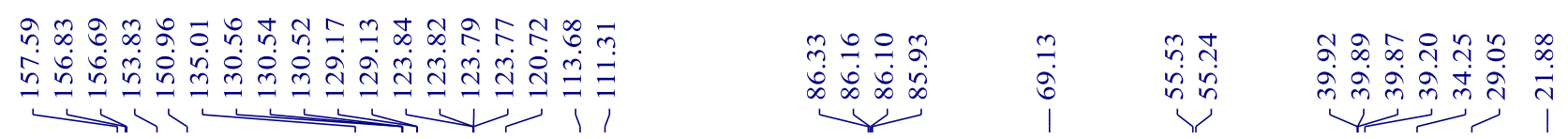

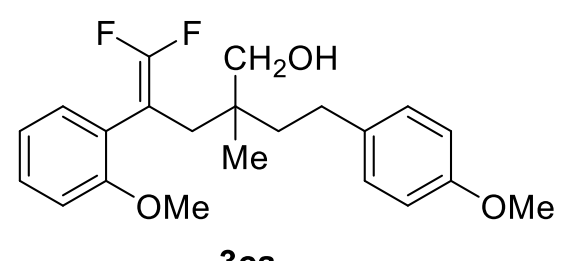

3ca

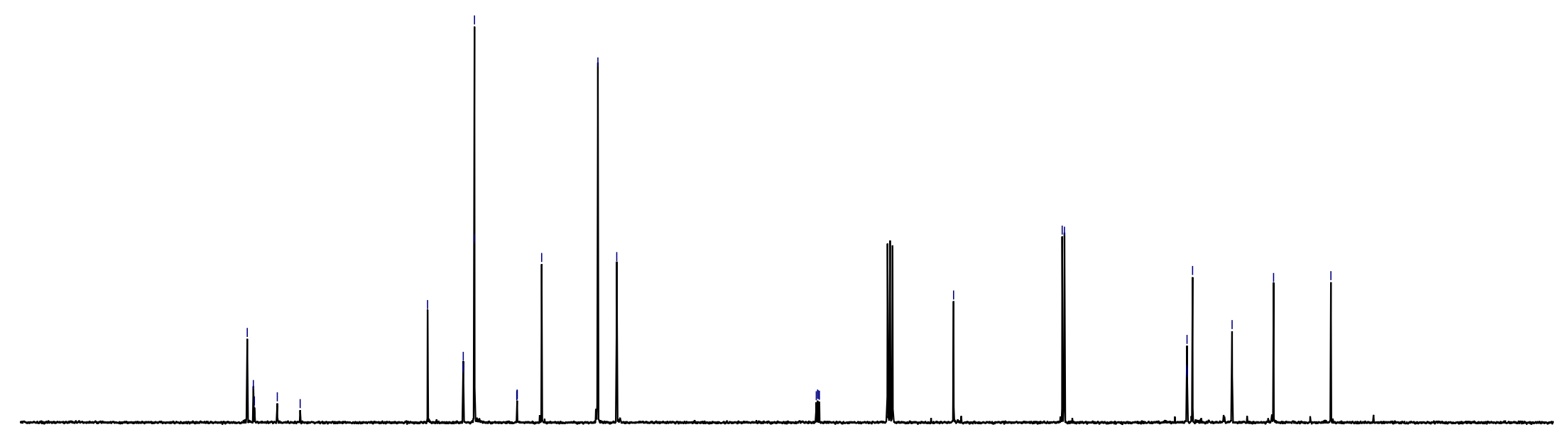


${ }^{19} \mathrm{~F}$ NMR-spectrum $\left(376 \mathrm{MHz}, \mathrm{CDCl}_{3}\right.$ ) of $\mathbf{3 c a}$
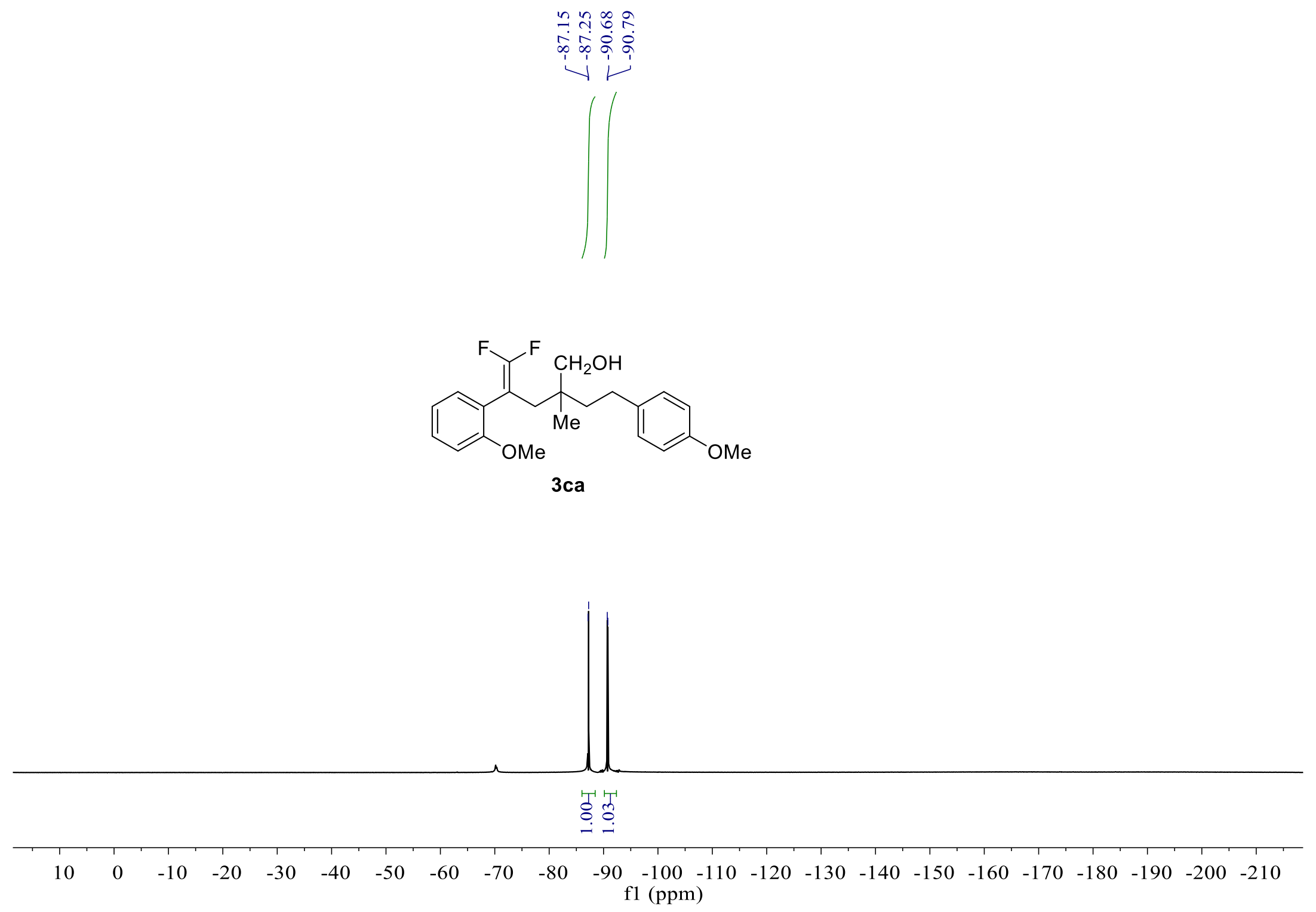
${ }^{1} \mathrm{H}$ NMR-spectrum (400 MHz, $\mathrm{CDCl}_{3}$ ) of 3da

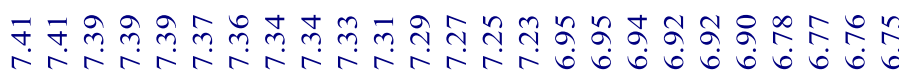

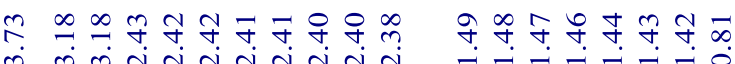

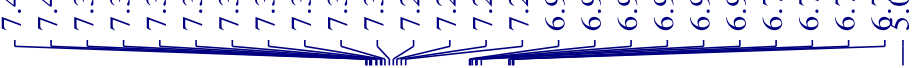

$i \sqrt{m i n g i n i v i n}$
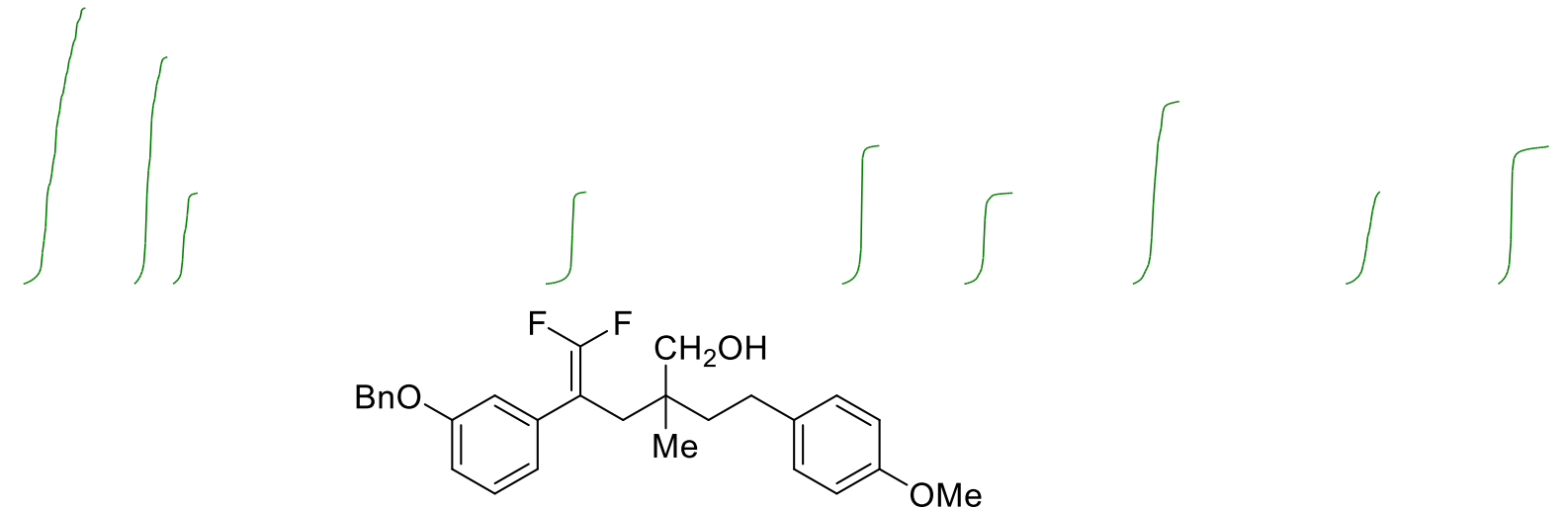

3da

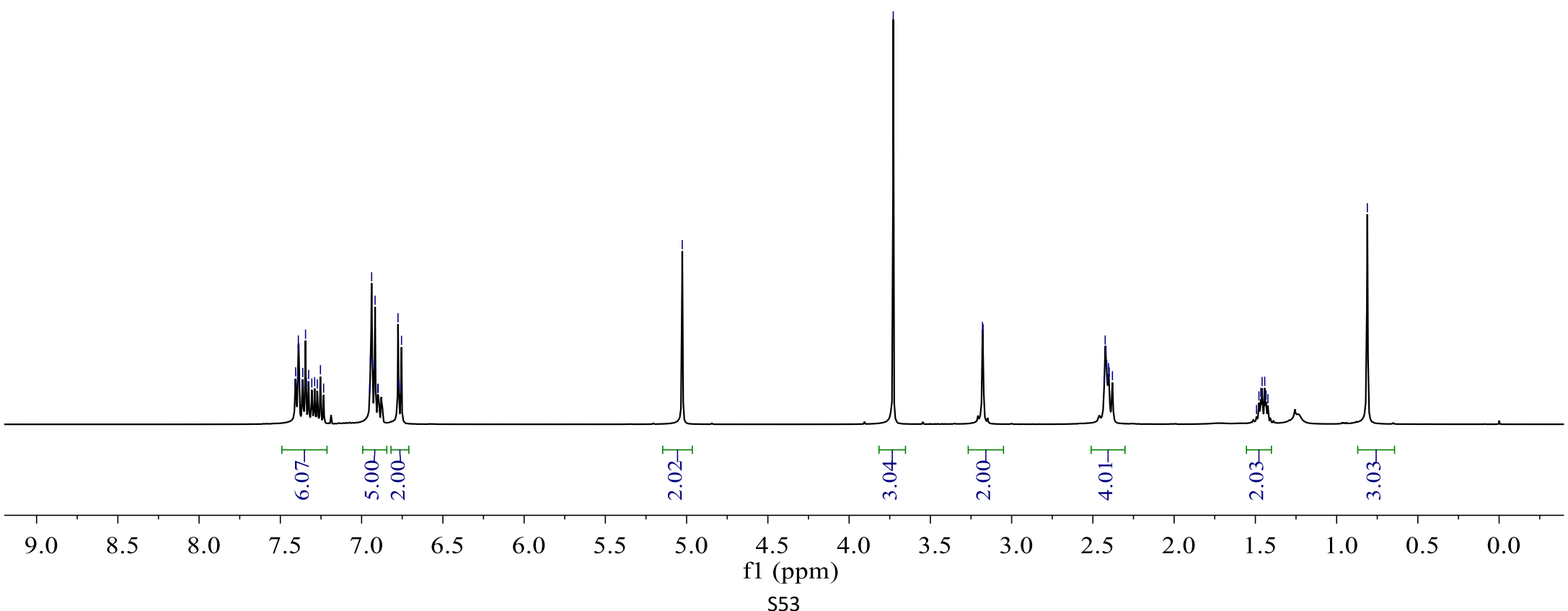

S53 

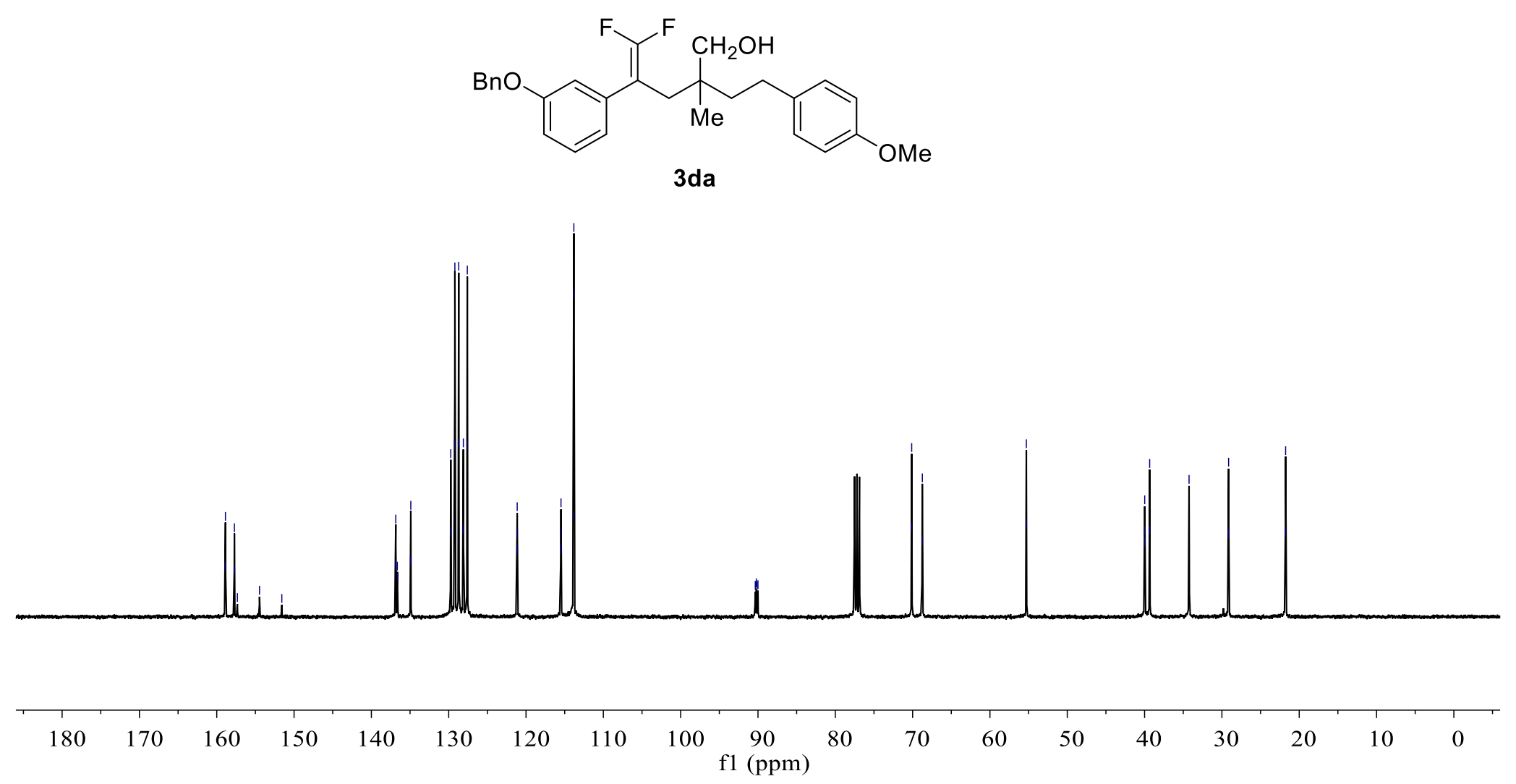
${ }^{19} \mathrm{~F}$ NMR-spectrum $\left(376 \mathrm{MHz}, \mathrm{CDCl}_{3}\right.$ ) of $\mathbf{3 d a}$

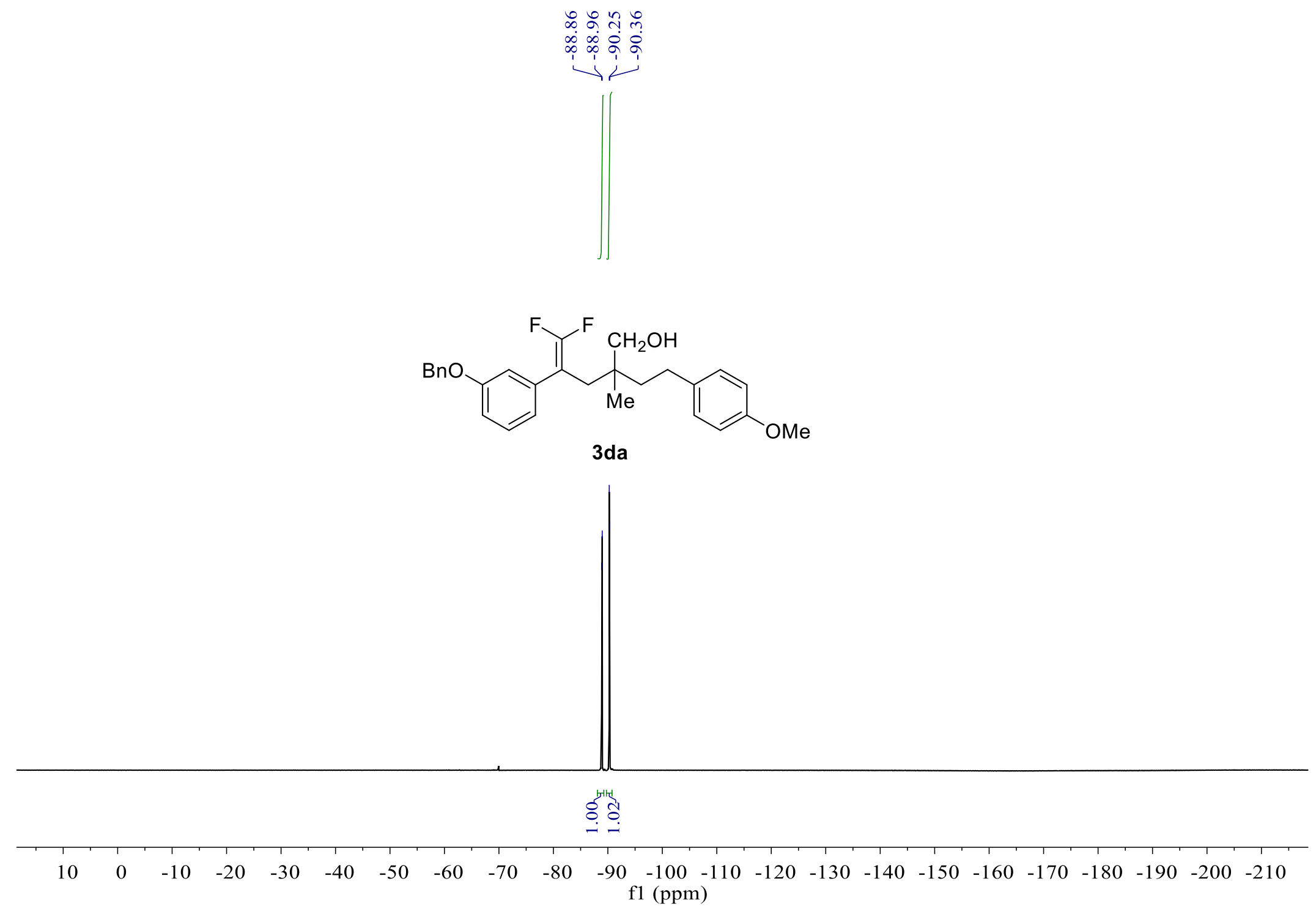


${ }^{1} \mathrm{H}$ NMR-spectrum $\left(400 \mathrm{MHz}, \mathrm{CDCl}_{3}\right)$ of $\mathbf{3 e a}$

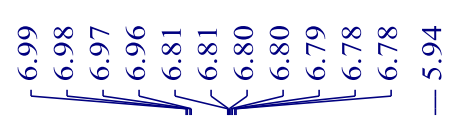

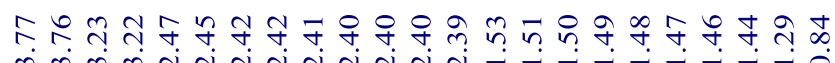

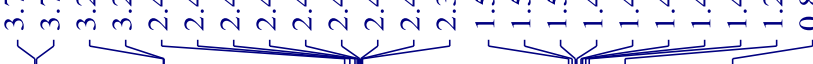
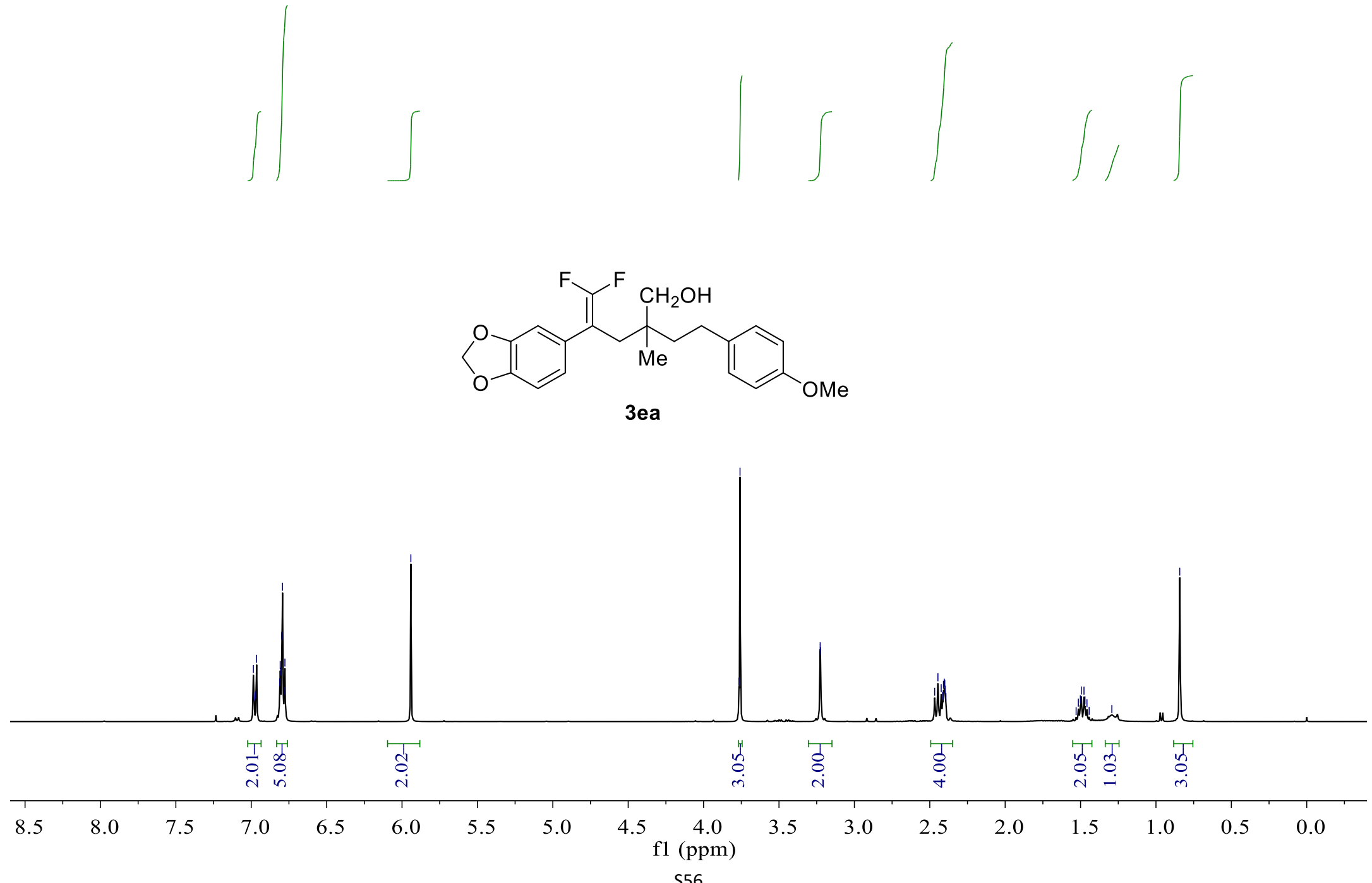

S56 

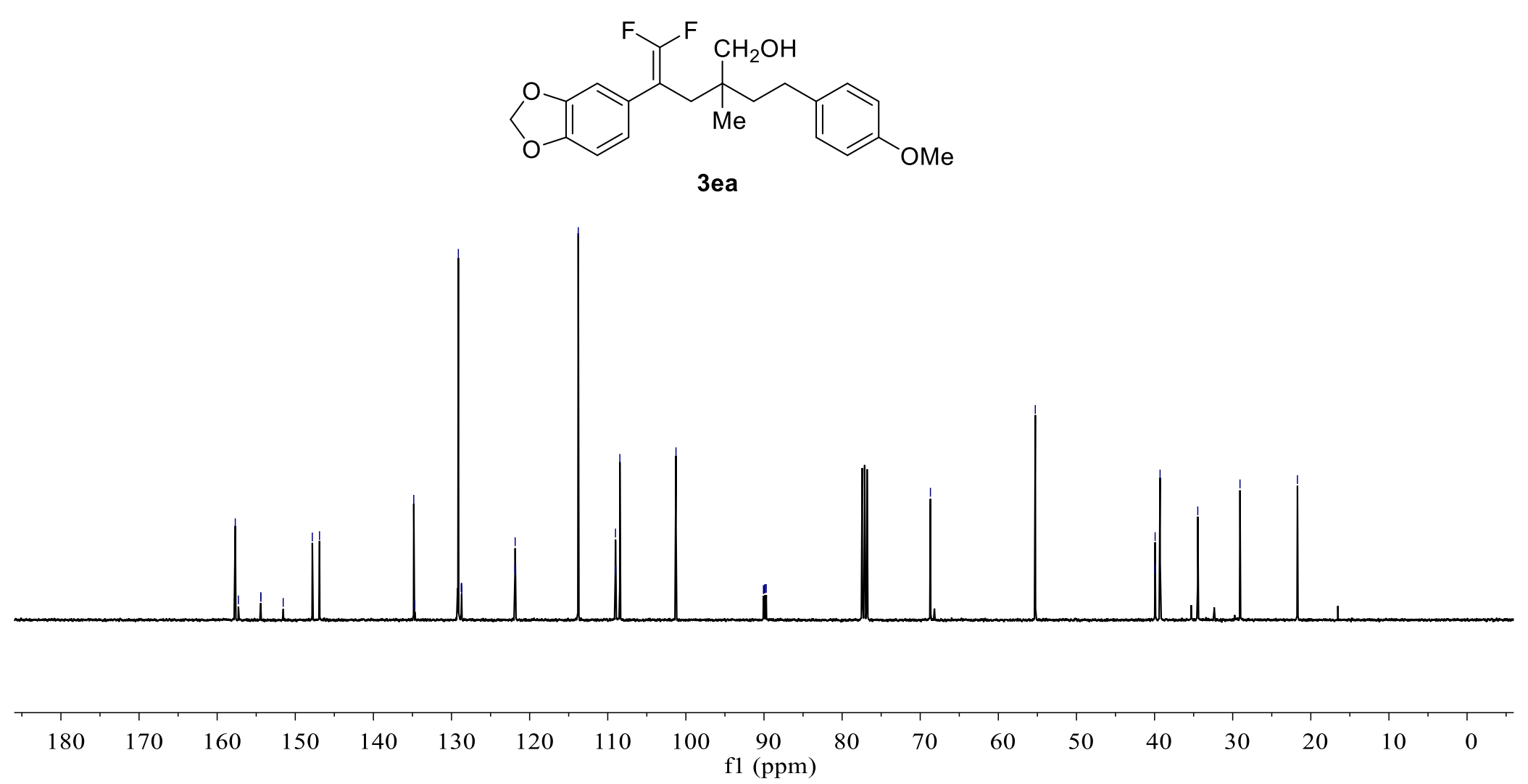
${ }^{19} \mathrm{~F}$ NMR-spectrum $\left(376 \mathrm{MHz}, \mathrm{CDCl}_{3}\right.$ ) of $\mathbf{3 e a}$
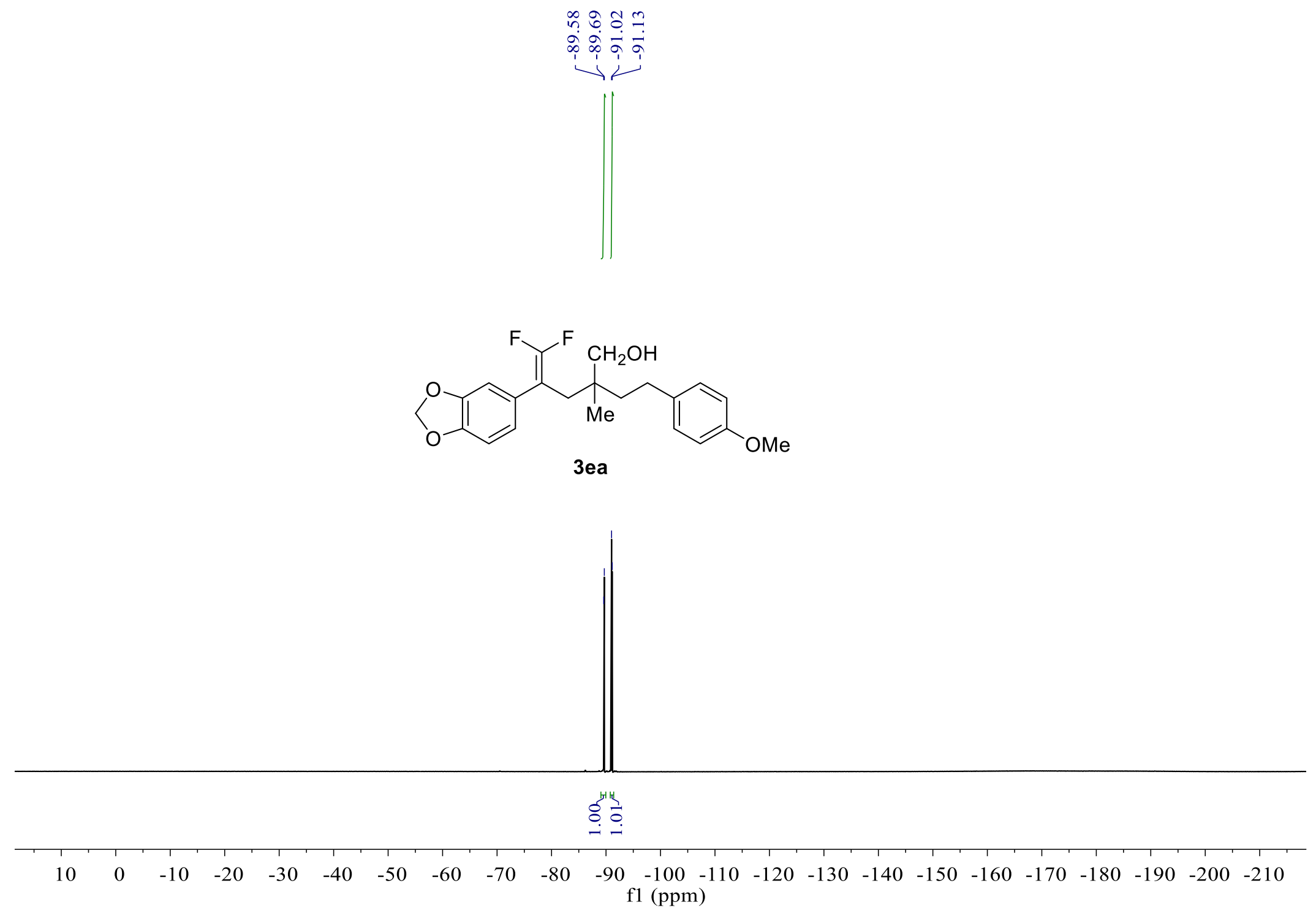
${ }^{1} \mathrm{H}$ NMR-spectrum $\left(400 \mathrm{MHz}, \mathrm{CDCl}_{3}\right)$ of $\mathbf{3 f a}$

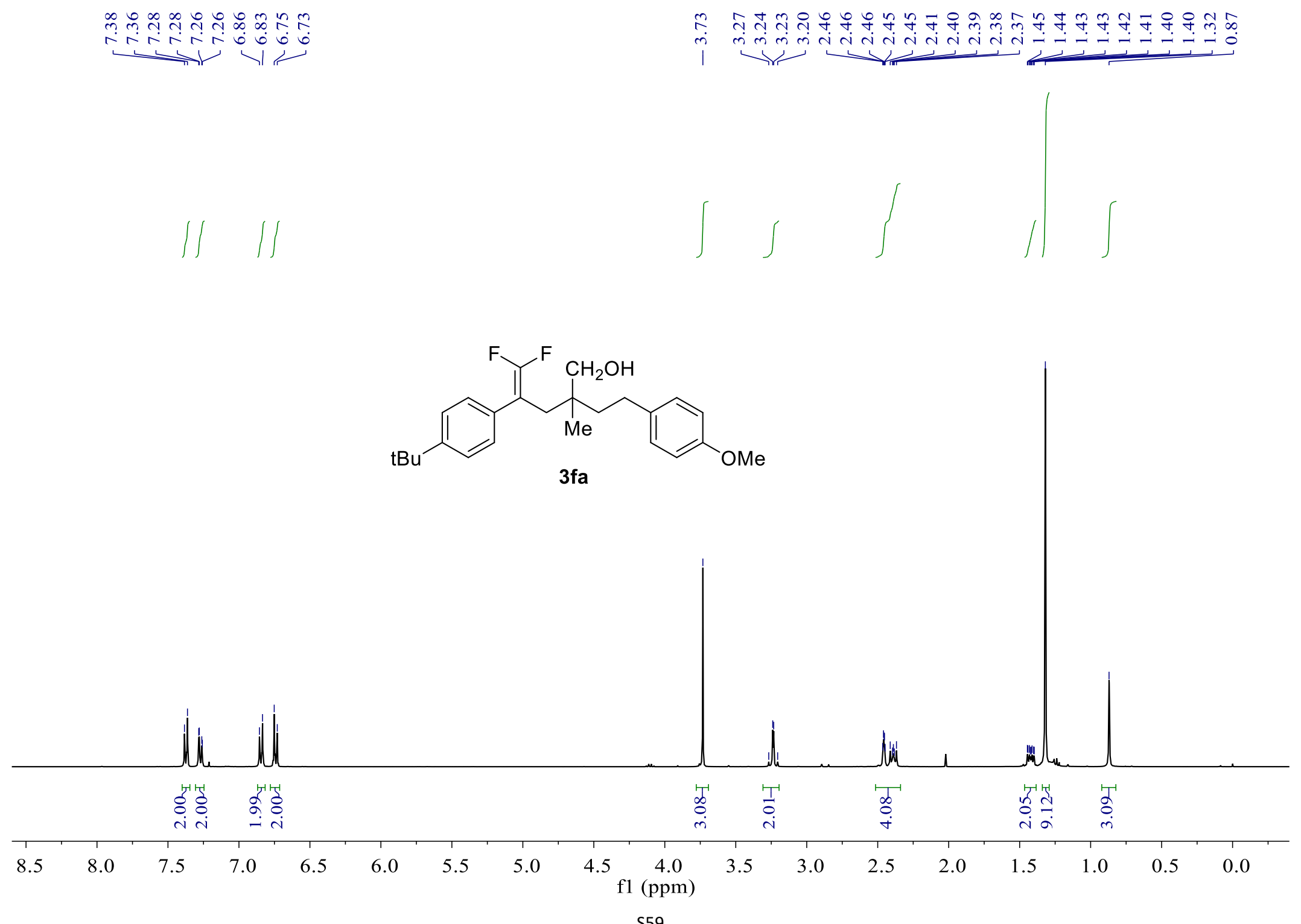

559 
${ }^{13} \mathrm{C}$ NMR-spectrum $\left(101 \mathrm{MHz}, \mathrm{CDCl}_{3}\right)$ of $\mathbf{3 f a}$
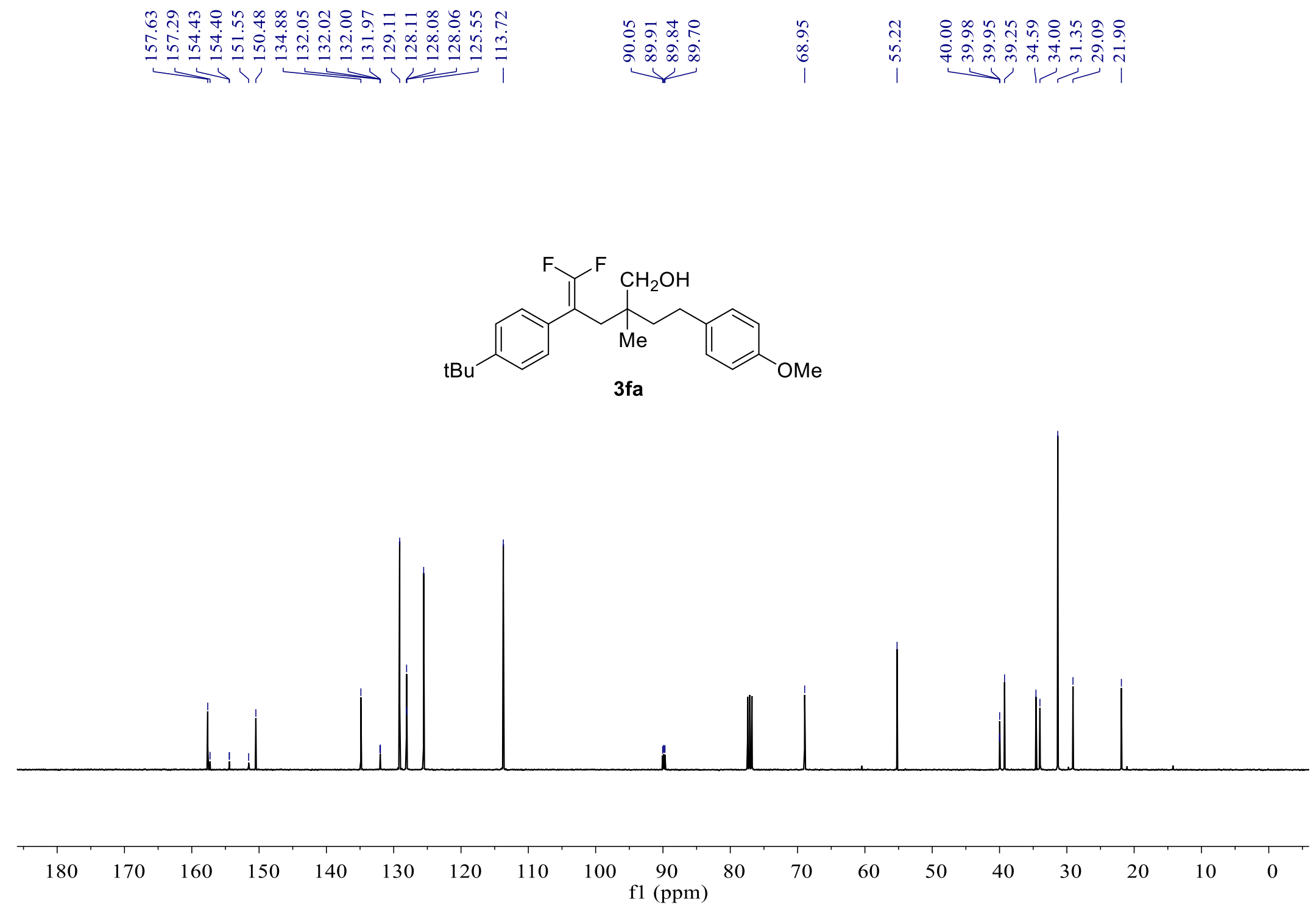
${ }^{19} \mathrm{~F} \mathrm{NMR}$-spectrum $\left(376 \mathrm{MHz}, \mathrm{CDCl}_{3}\right.$ ) of $\mathbf{3 f a}$

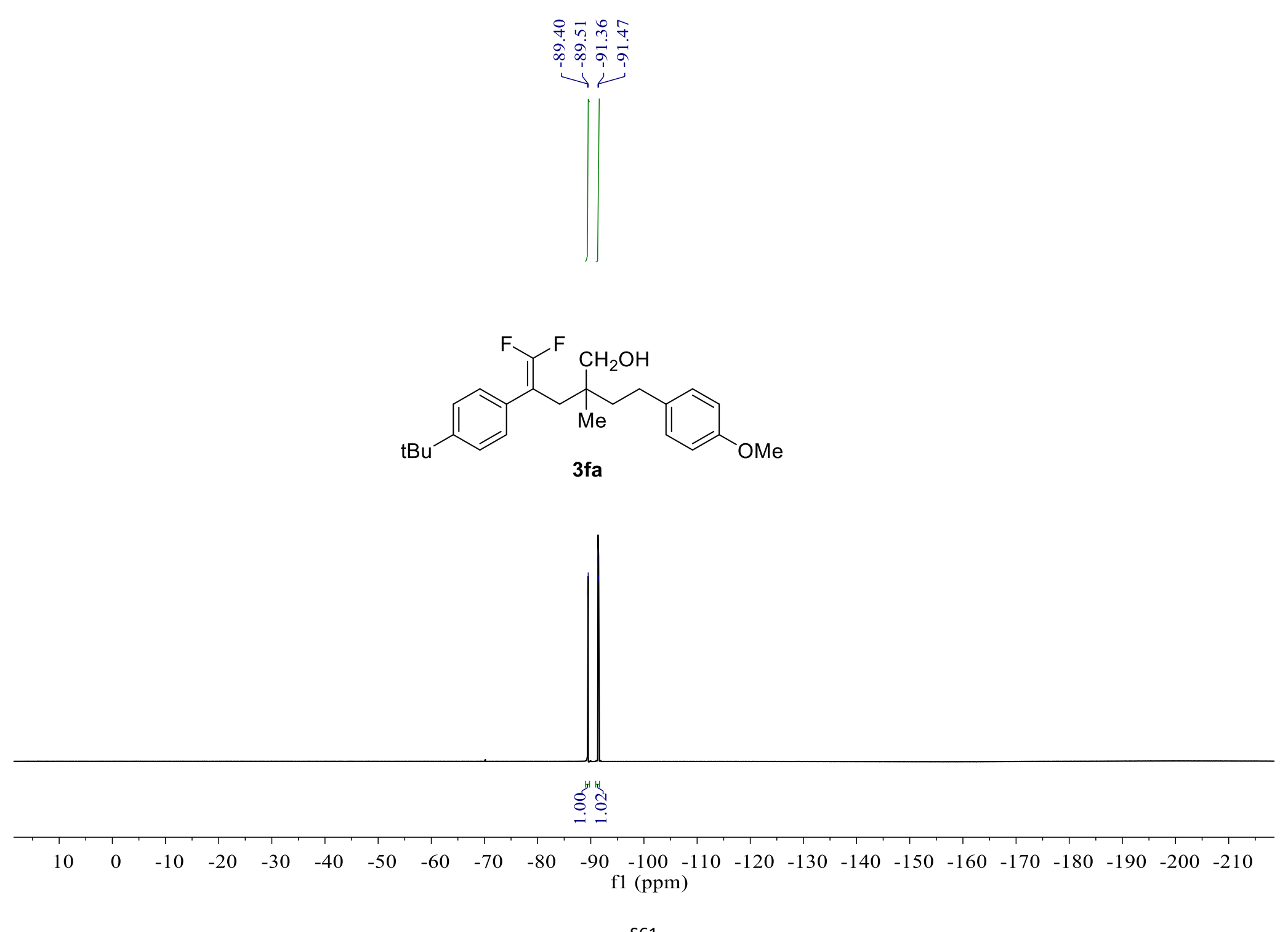


${ }^{1} \mathrm{H}$ NMR-spectrum (400 MHz, $\mathrm{CDCl}_{3}$ ) of $\mathbf{3 g a}$

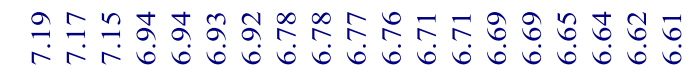

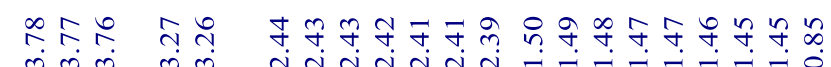

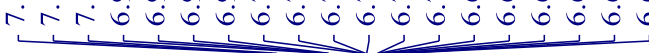

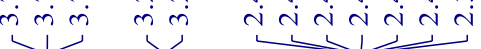
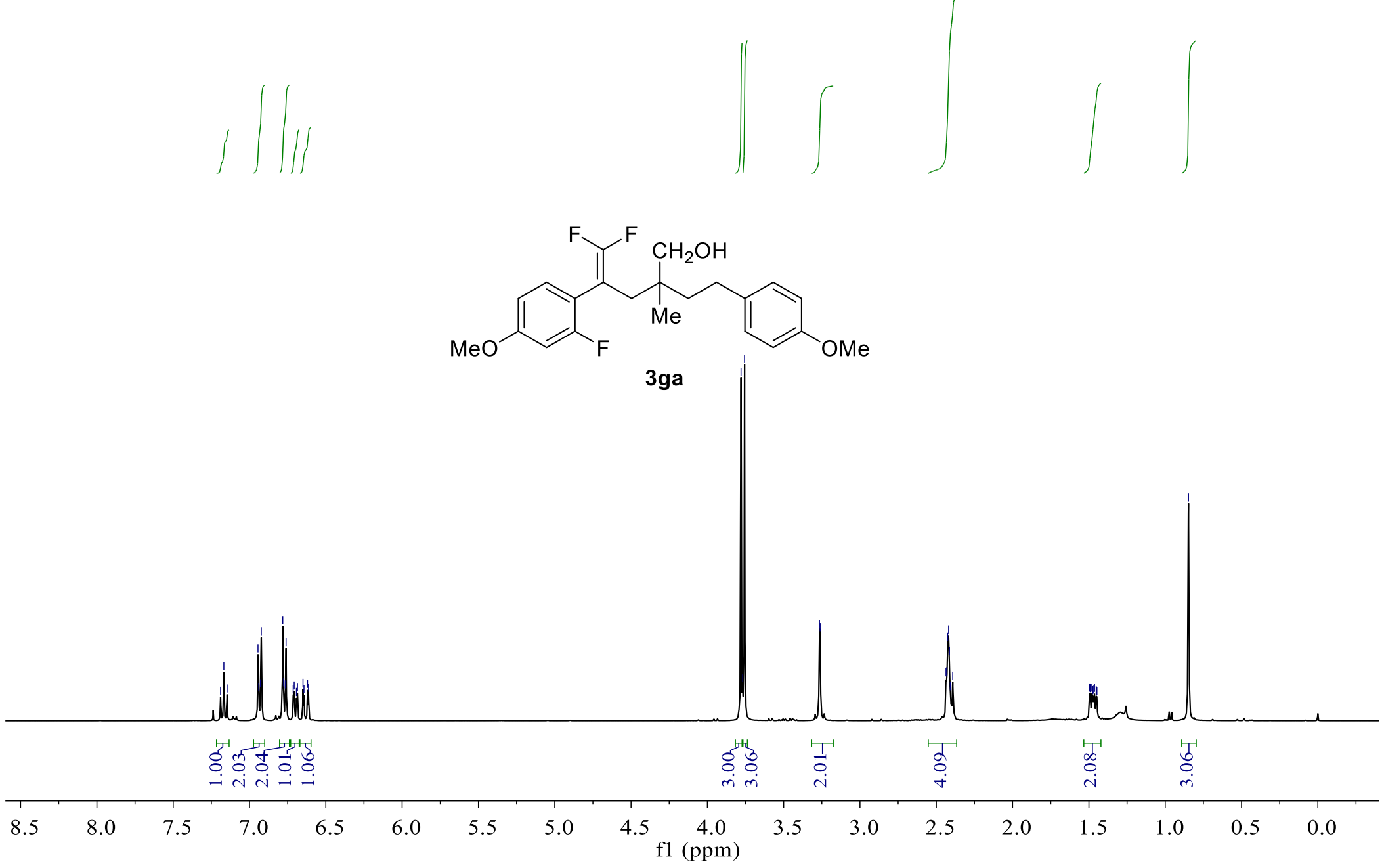

562 
${ }^{13} \mathrm{C}$ NMR-spectrum $\left(101 \mathrm{MHz}, \mathrm{CDCl}_{3}\right)$ of $\mathbf{3 g a}$
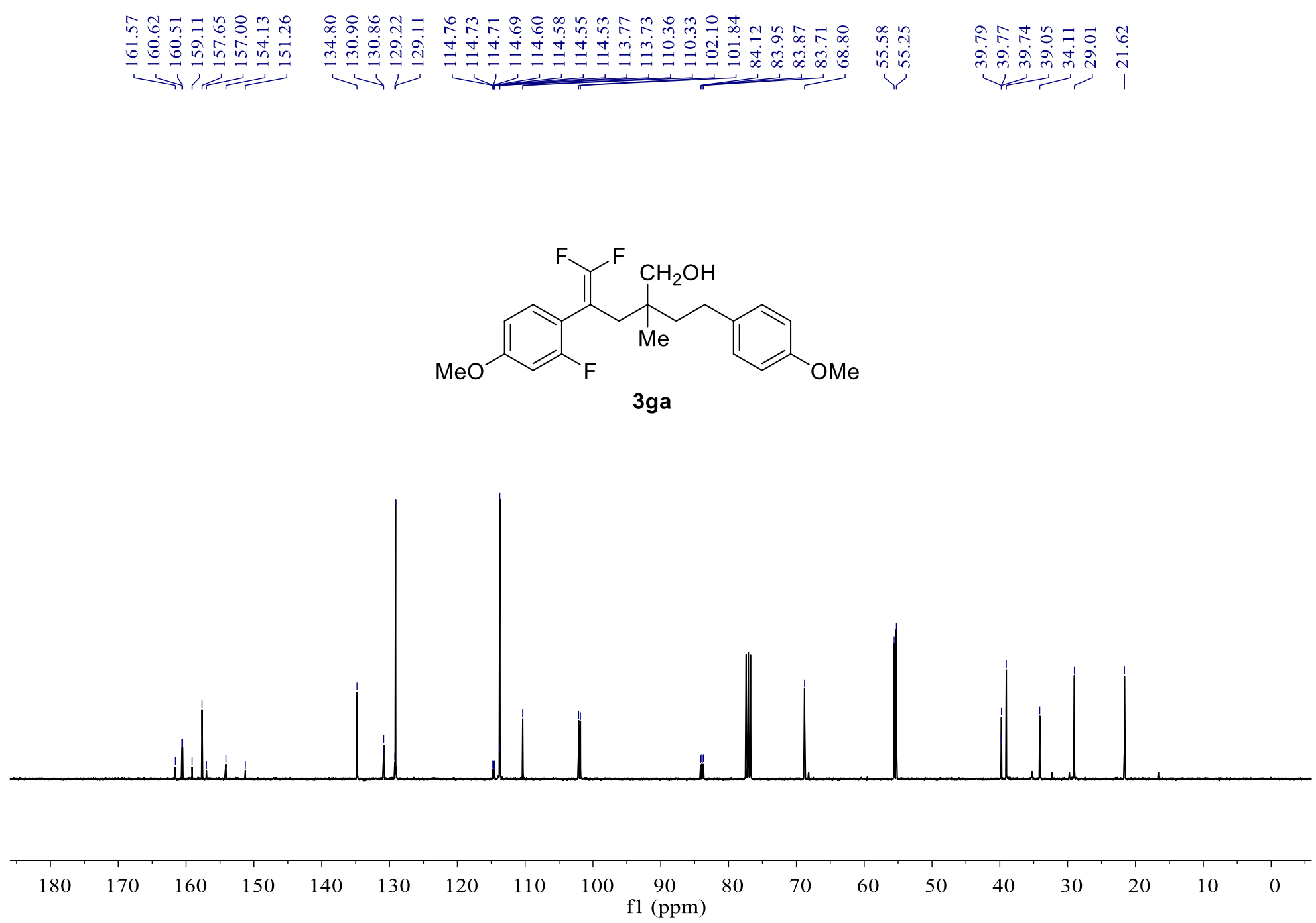
${ }^{19} \mathrm{~F} \mathrm{NMR}$-spectrum $\left(376 \mathrm{MHz}, \mathrm{CDCl}_{3}\right)$ of $\mathbf{3 g a}$
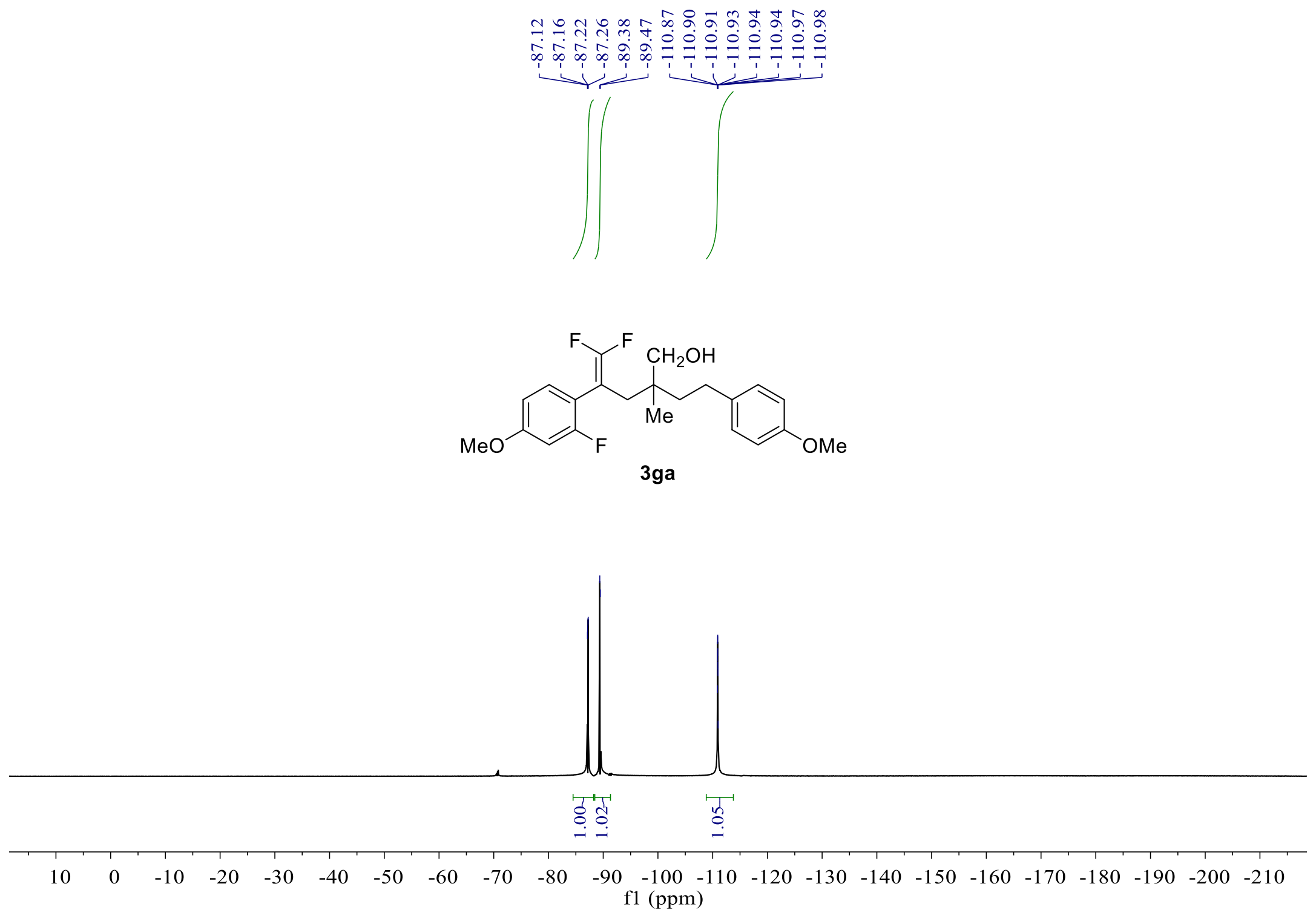
${ }^{1} \mathrm{H}$ NMR-spectrum $\left(400 \mathrm{MHz}, \mathrm{CDCl}_{3}\right)$ of $\mathbf{3 h a}$

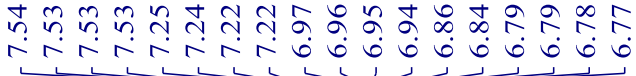

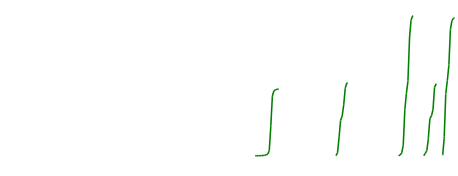

il
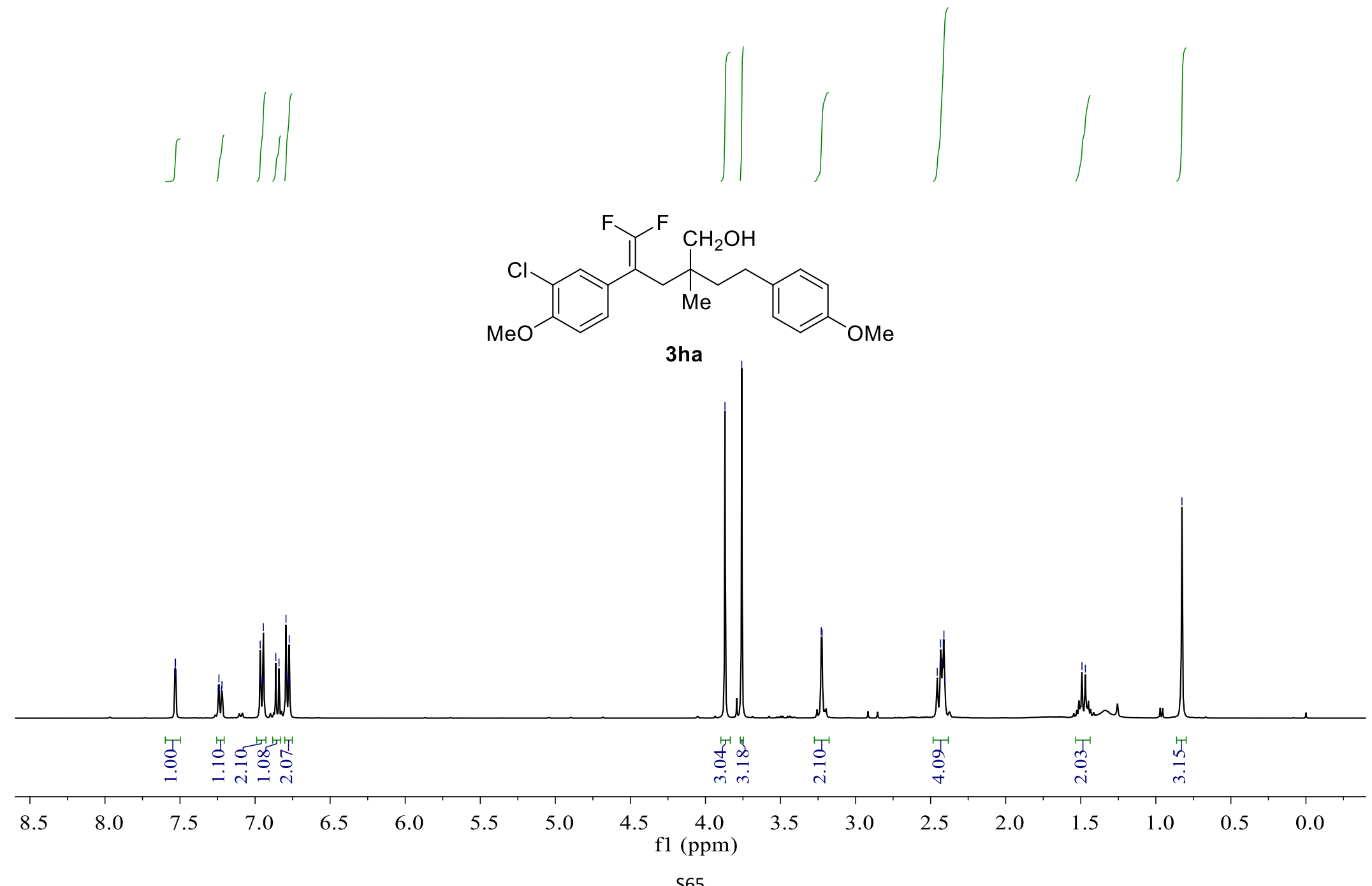

S65 
${ }^{13} \mathrm{C}$ NMR-spectrum $\left(101 \mathrm{MHz}, \mathrm{CDCl}_{3}\right)$ of $3 \mathbf{h a}$

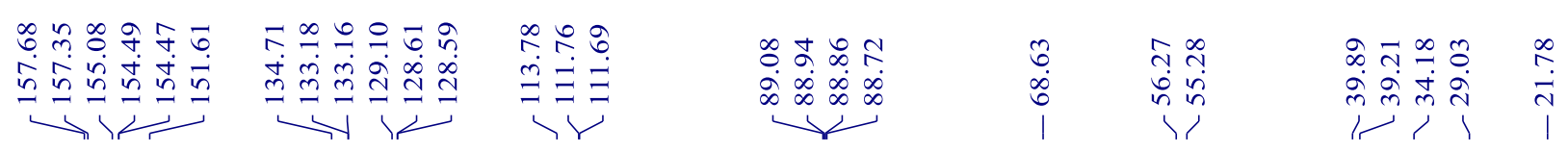

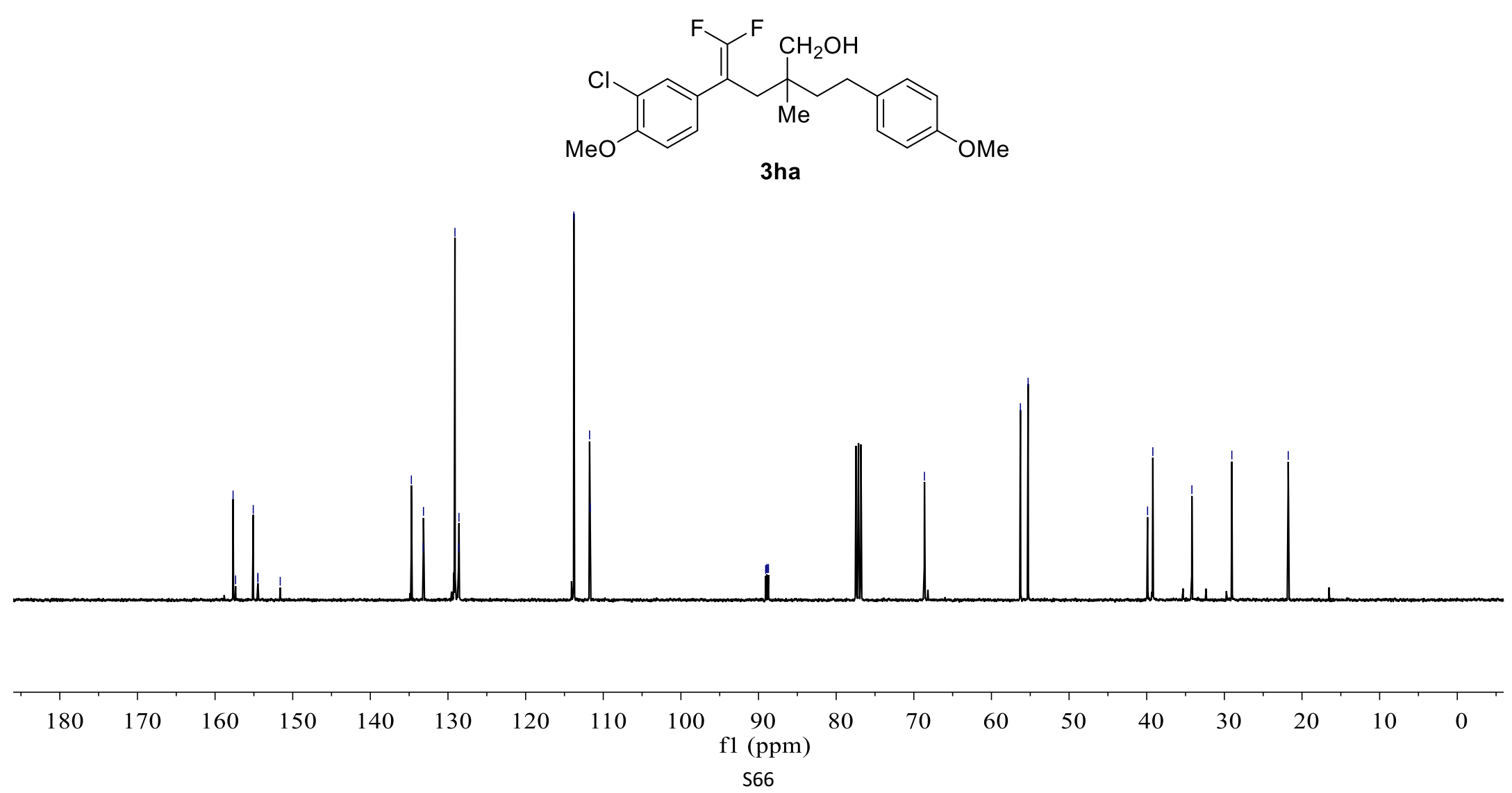


${ }^{19} \mathrm{~F}$ NMR-spectrum $\left(376 \mathrm{MHz}, \mathrm{CDCl}_{3}\right)$ of $\mathbf{3 h a}$
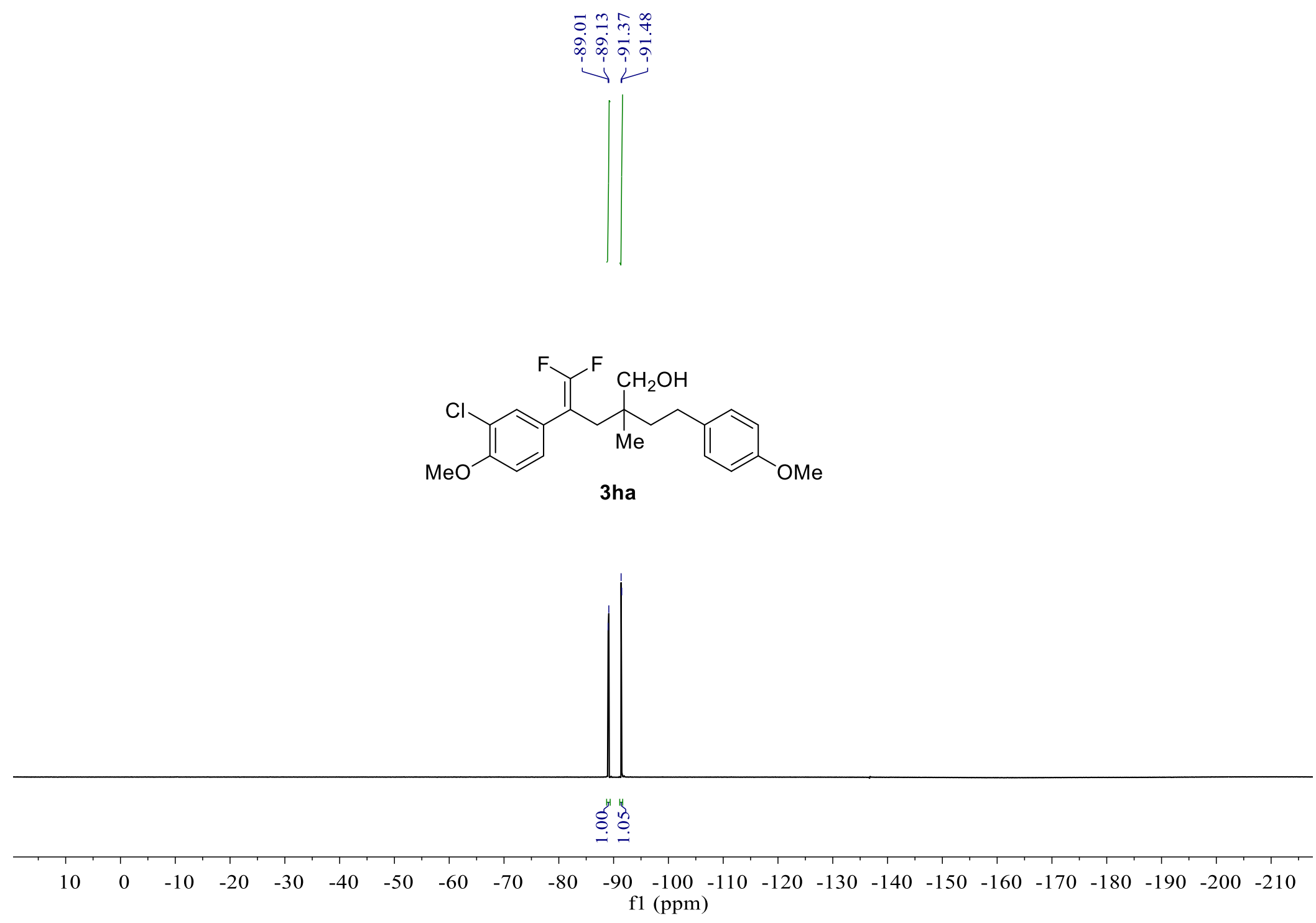

S67 
${ }^{1} \mathrm{H}$ NMR-spectrum $\left(400 \mathrm{MHz}, \mathrm{CDCl}_{3}\right)$ of 3ia

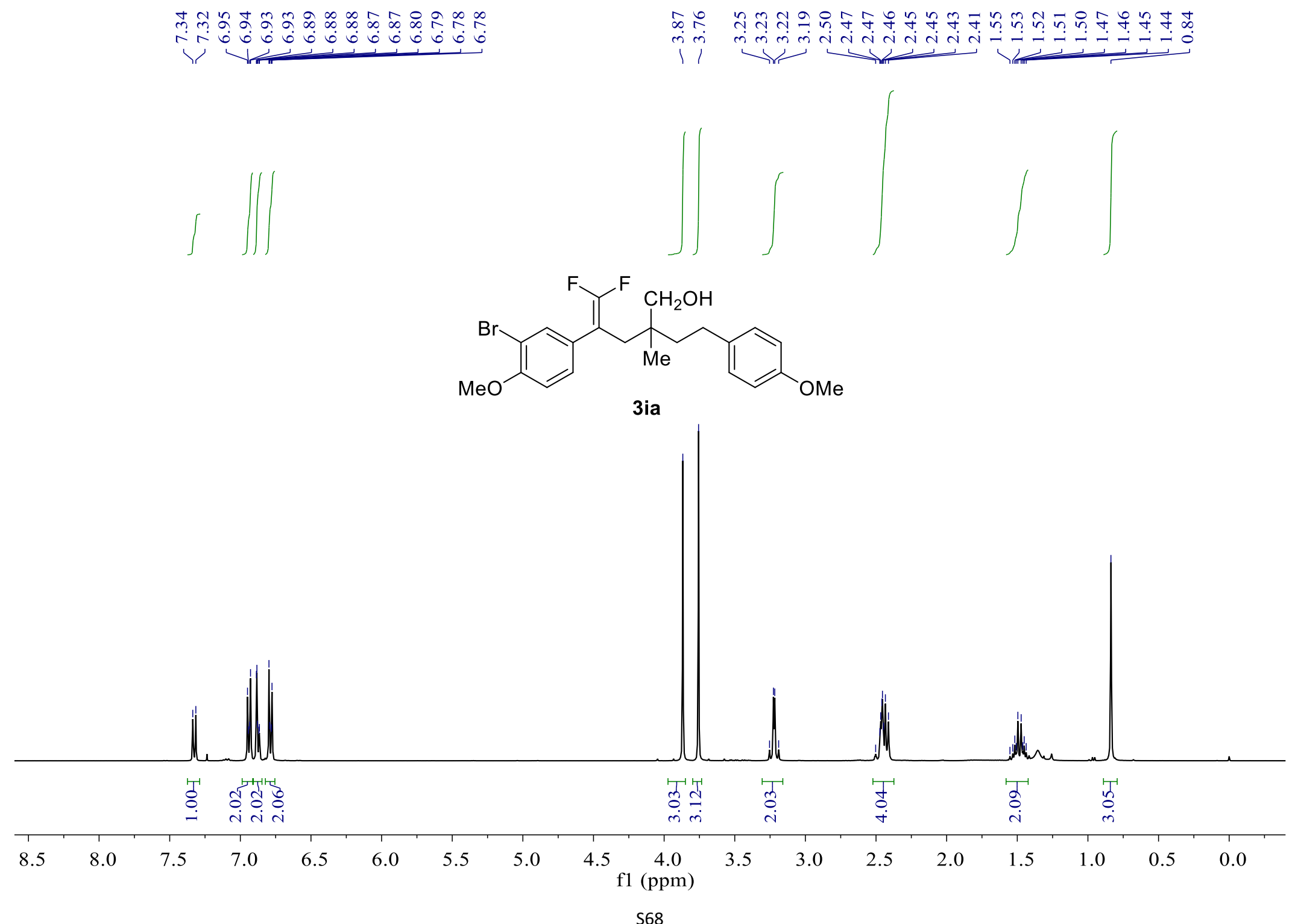


${ }^{13} \mathrm{C}$ NMR-spectrum $\left(101 \mathrm{MHz}, \mathrm{CDCl}_{3}\right)$ of 3ia 

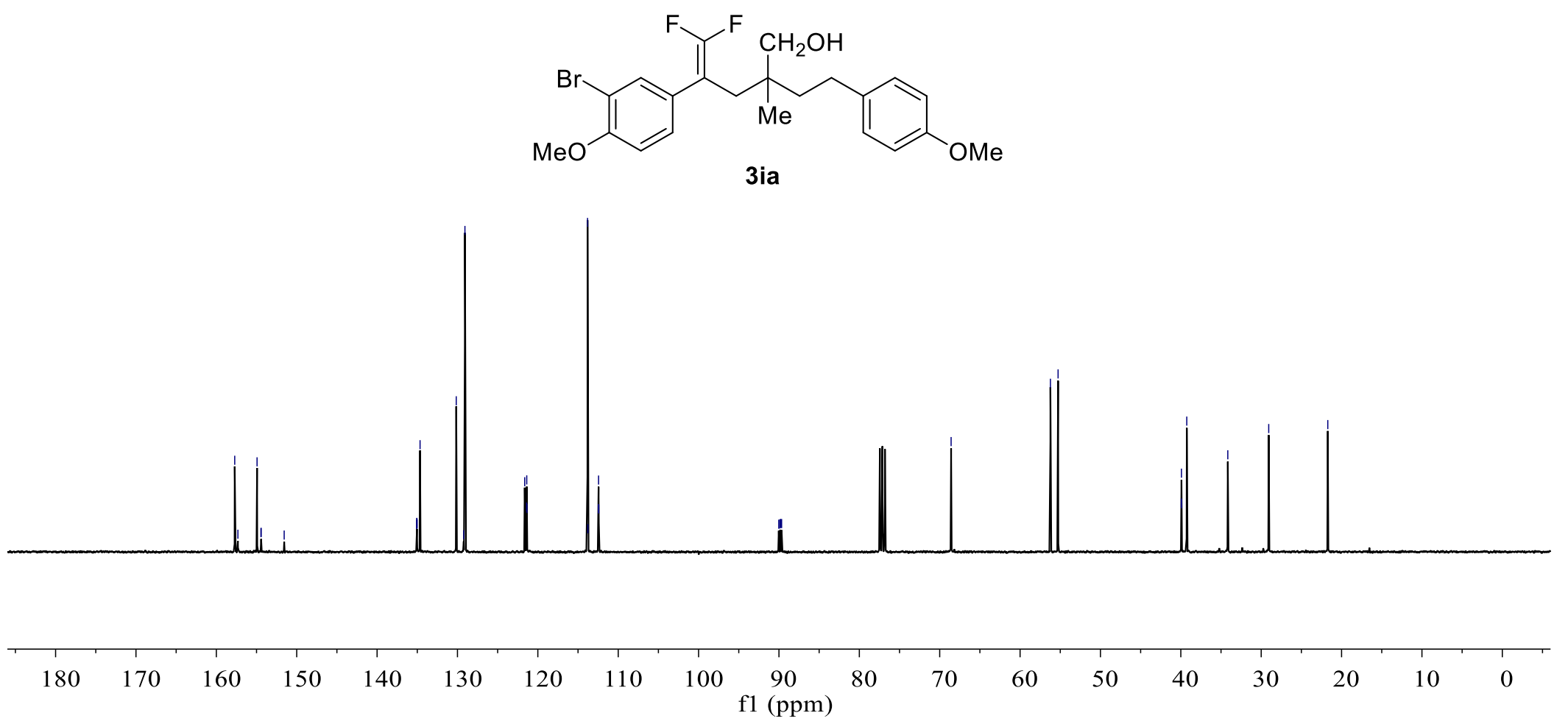

${ }^{19} \mathrm{~F}$ NMR-spectrum $\left(376 \mathrm{MHz}, \mathrm{CDCl}_{3}\right)$ of 3 ia 

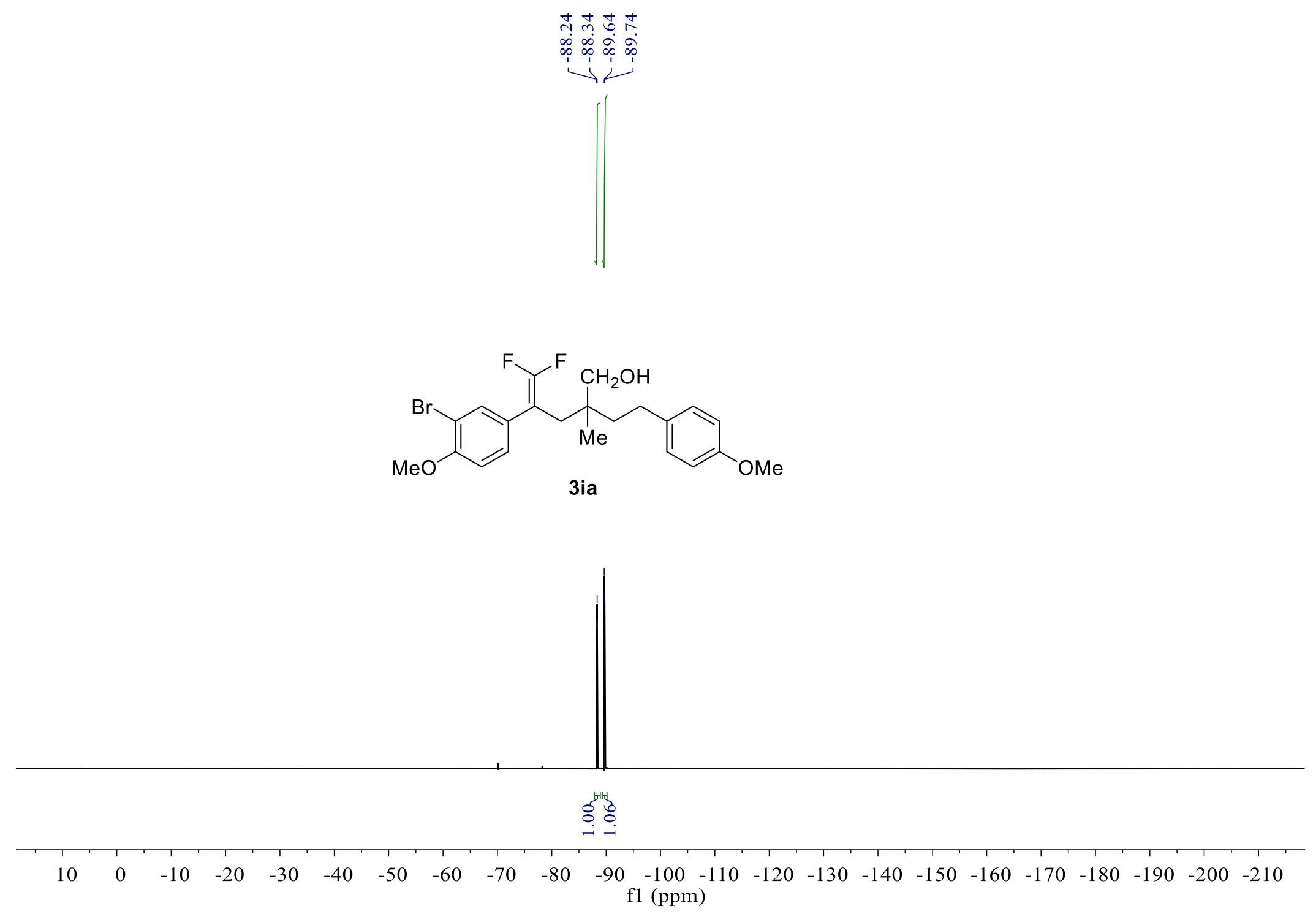

${ }^{1} \mathrm{H}$ NMR-spectrum $\left(400 \mathrm{MHz}, \mathrm{CDCl}_{3}\right.$ ) of $\mathbf{3 j a}$ 


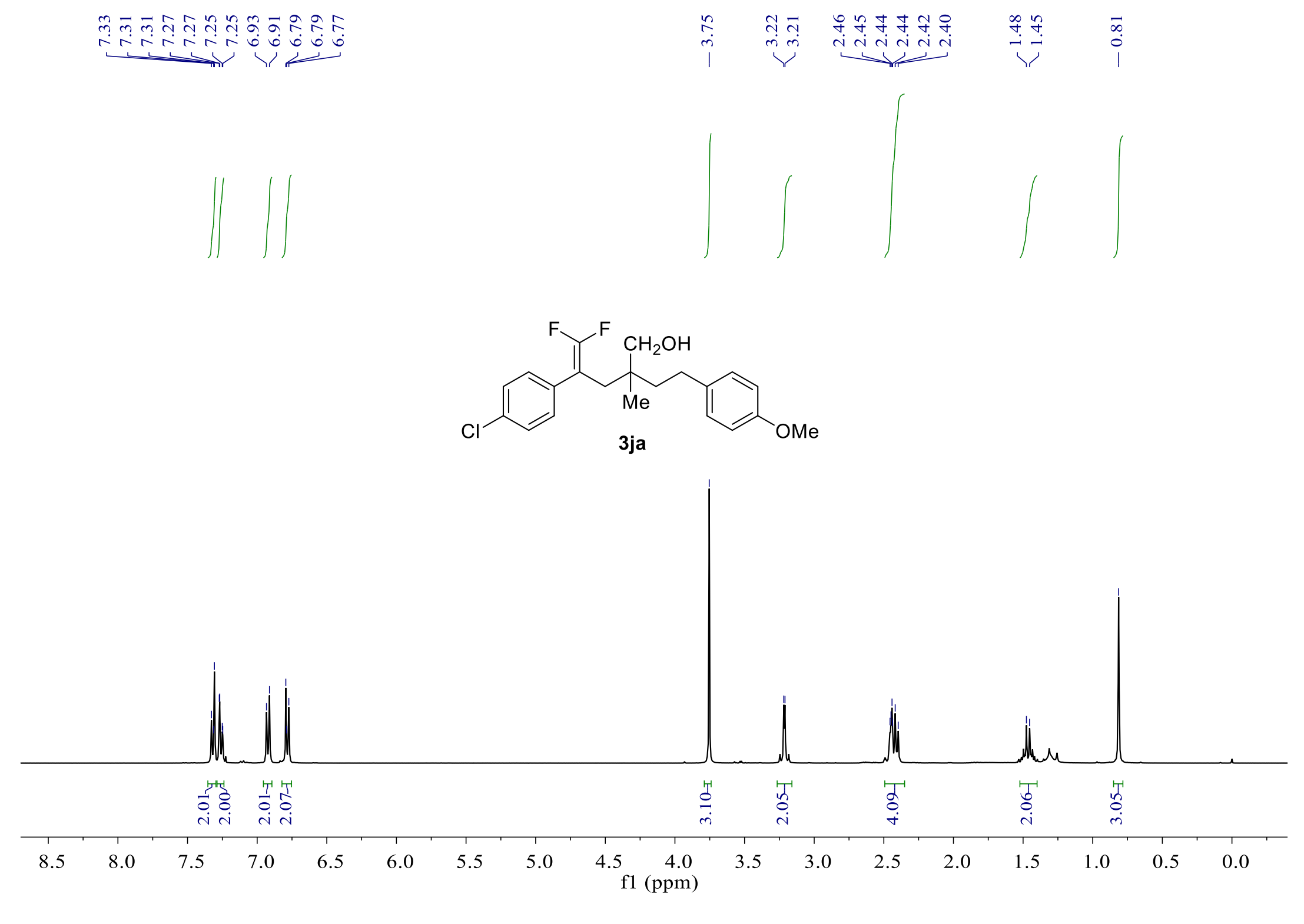


${ }^{13} \mathrm{C}$ NMR-spectrum $\left(101 \mathrm{MHz}, \mathrm{CDCl}_{3}\right)$ of $\mathbf{3 j a}$
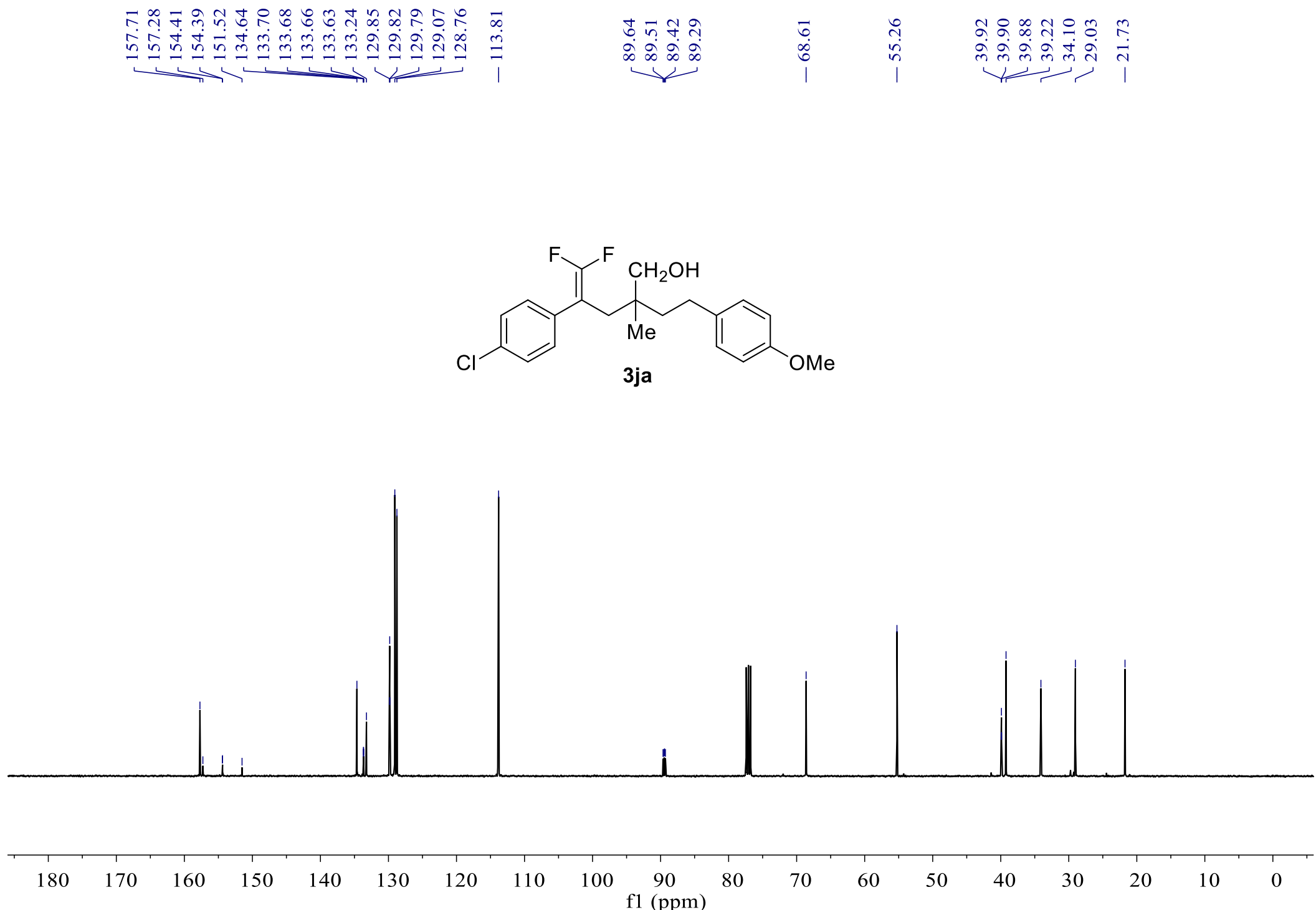

S73 
${ }^{19} \mathrm{~F} \mathrm{NMR}$-spectrum $\left(376 \mathrm{MHz}, \mathrm{CDCl}_{3}\right)$ of $\mathbf{3 j a}$
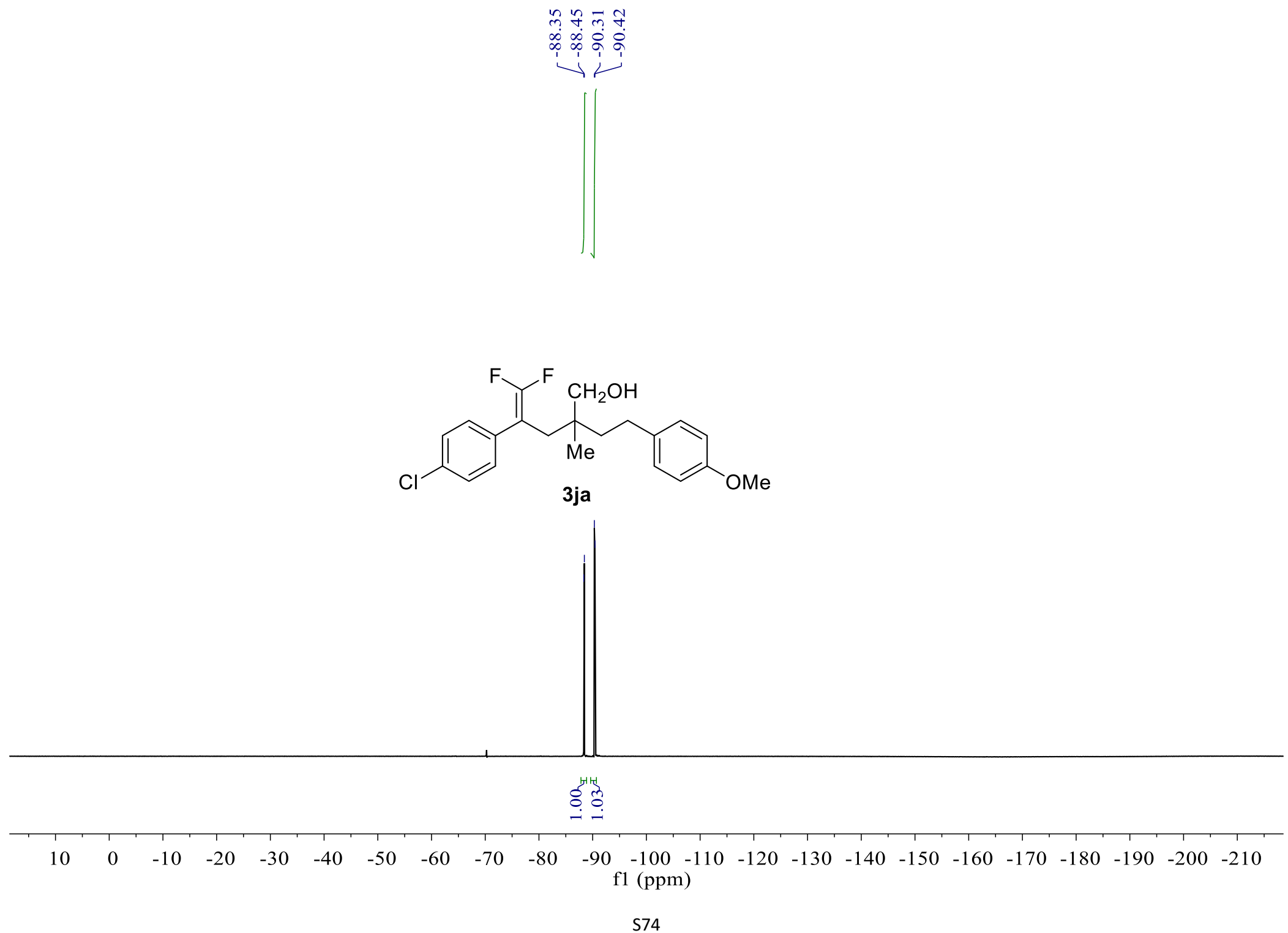


\section{${ }^{1} \mathrm{H}$ NMR-spectrum $\left(400 \mathrm{MHz}, \mathrm{CDCl}_{3}\right)$ of $\mathbf{3 k a}$}

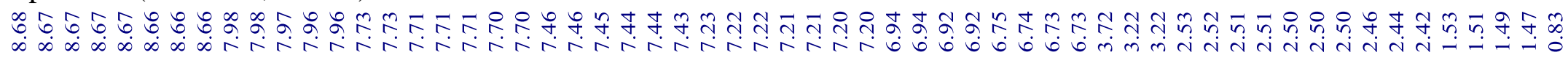

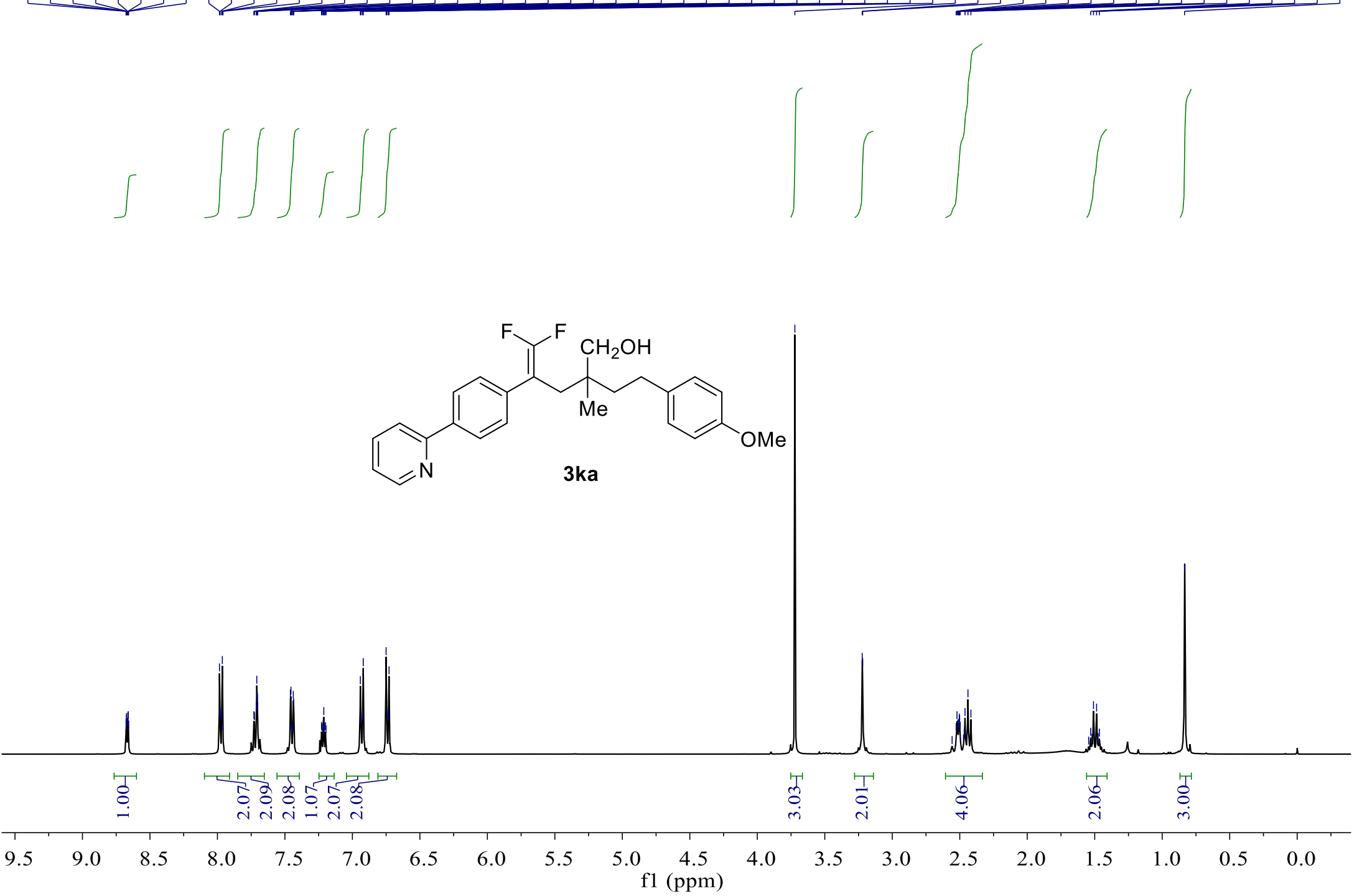


${ }^{13} \mathrm{C}$ NMR-spectrum $\left(101 \mathrm{MHz}, \mathrm{CDCl}_{3}\right)$ of $3 \mathbf{k a}$

\begin{tabular}{|c|c|c|c|c|}
\hline 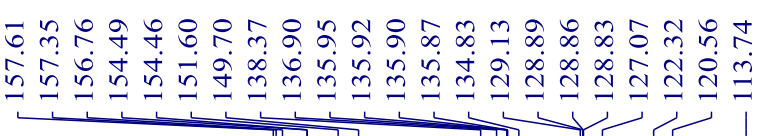 & 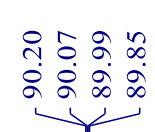 & $\begin{array}{c}\dot{*} \\
n \\
\infty \\
0 \\
1\end{array}$ & 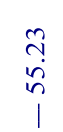 & 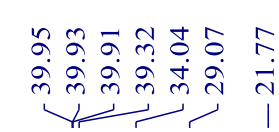 \\
\hline
\end{tabular}
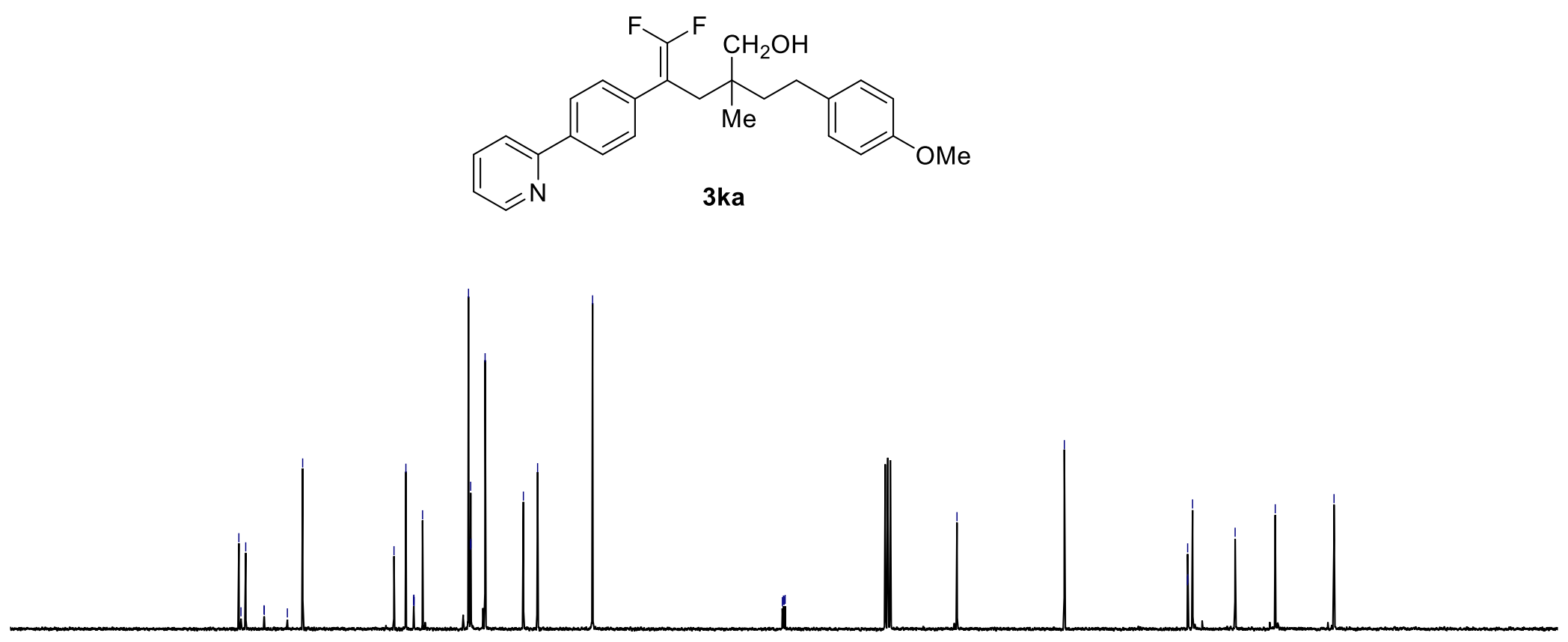

$\begin{array}{lllllllllllllllllll}180 & 170 & 160 & 150 & 140 & 130 & 120 & 110 & 100 & \begin{array}{c}90 \\ \mathrm{f} 1(\mathrm{ppm})\end{array} & 80 & 70 & 60 & 50 & 40 & 30 & 20 & 10 & 0\end{array}$ 


\section{${ }^{19}$ F NMR-spectrum (376 MHz, $\mathrm{CDCl}_{3}$ ) of 3ka}
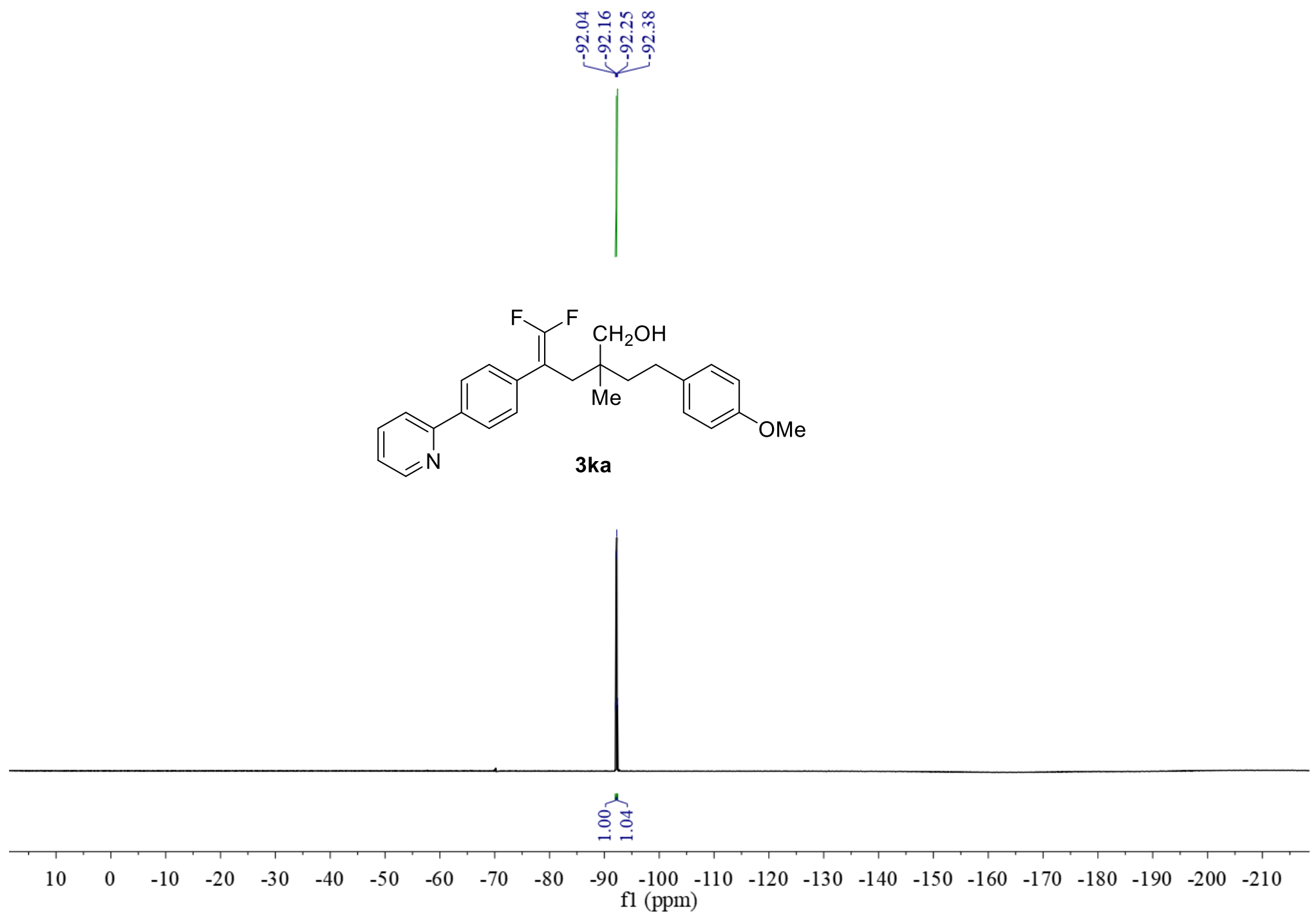
${ }^{1} \mathrm{H}$ NMR-spectrum $\left(400 \mathrm{MHz}, \mathrm{CDCl}_{3}\right)$ of 3la

桴
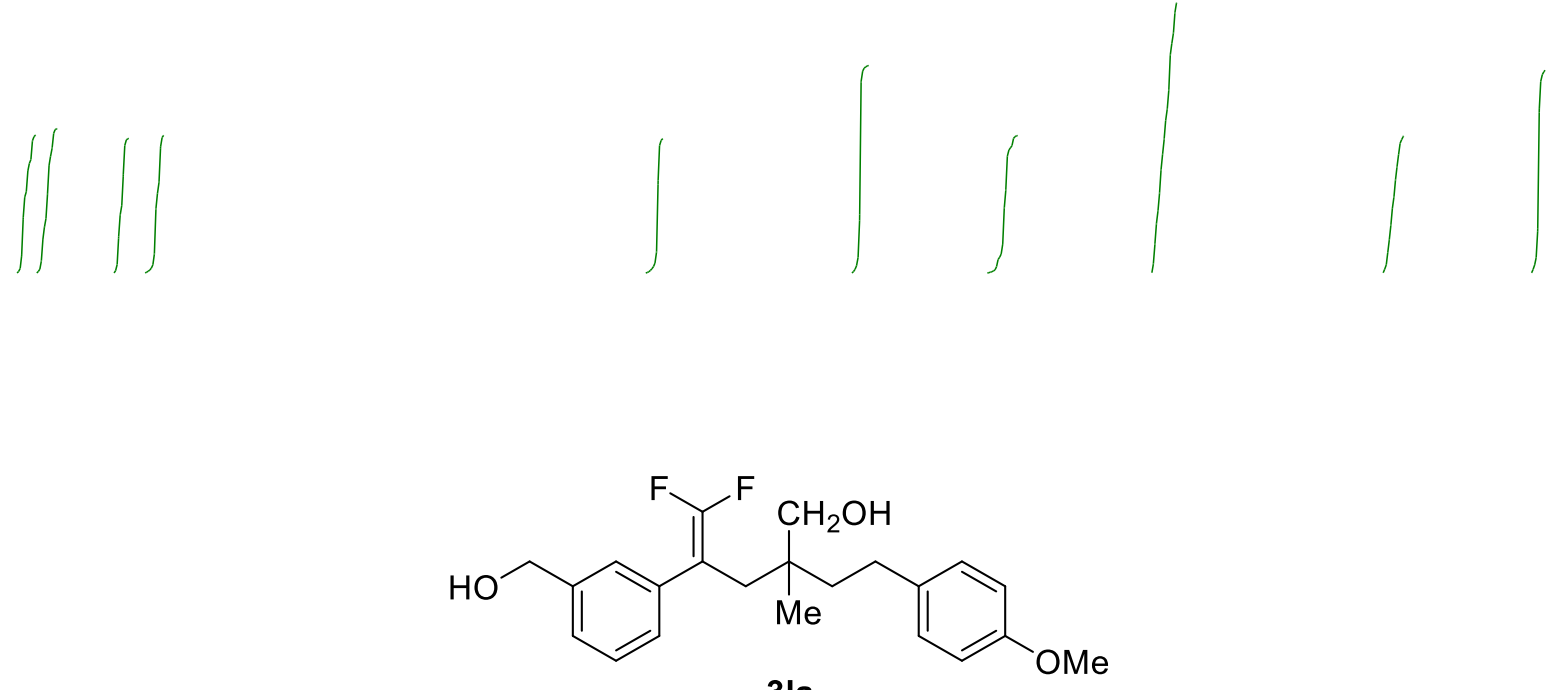

31

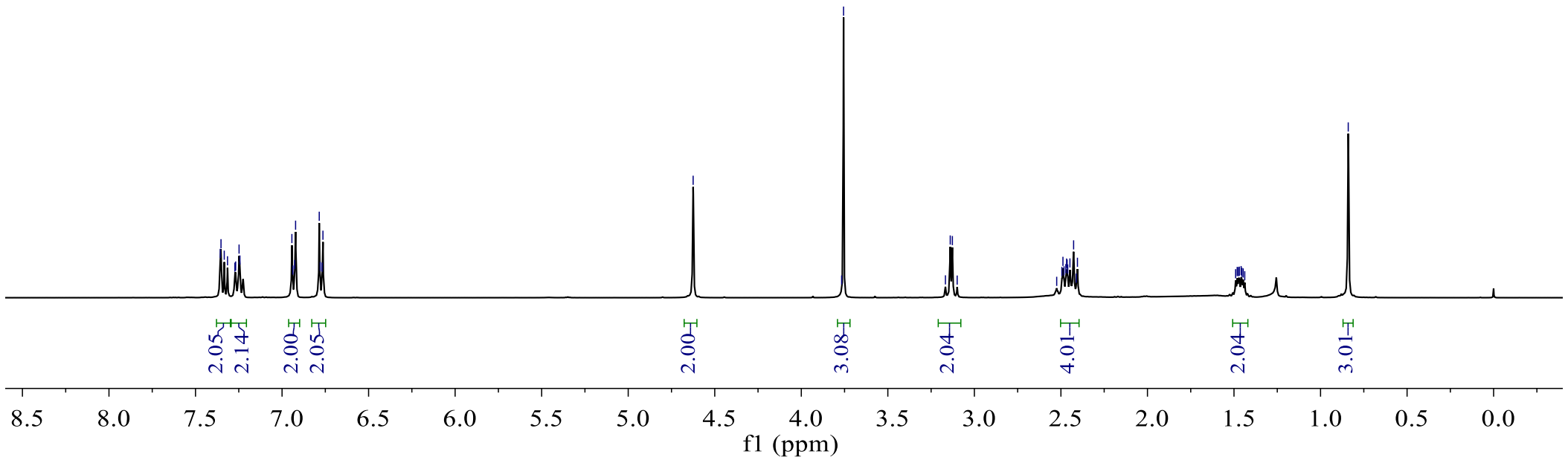


${ }^{13} \mathrm{C}$ NMR-spectrum $\left(101 \mathrm{MHz}, \mathrm{CDCl}_{3}\right)$ of 3la

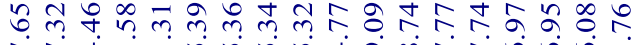

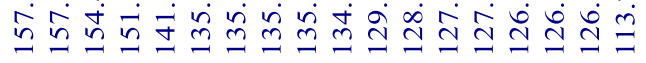

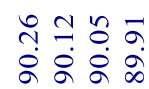

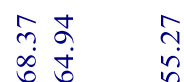
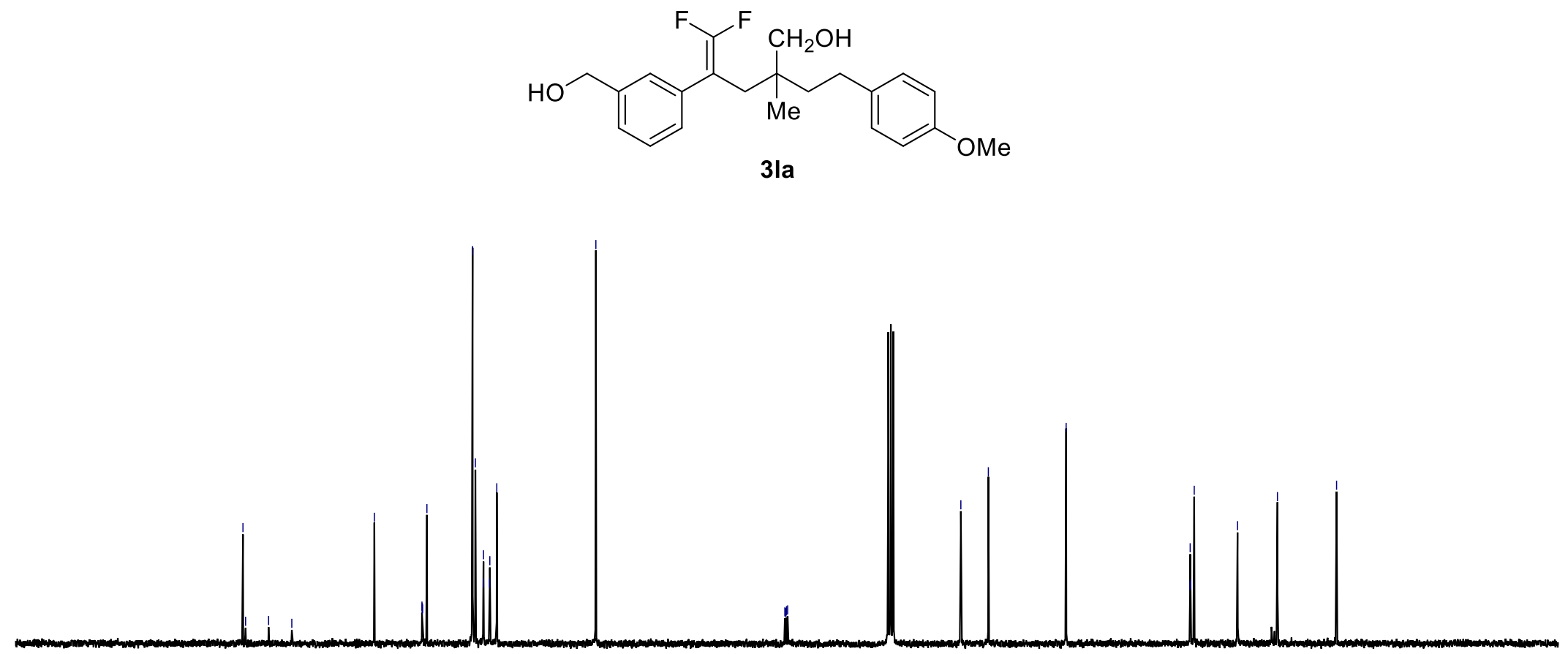
${ }^{19} \mathrm{~F} \mathrm{NMR}$-spectrum $\left(376 \mathrm{MHz}, \mathrm{CDCl}_{3}\right)$ of 3la
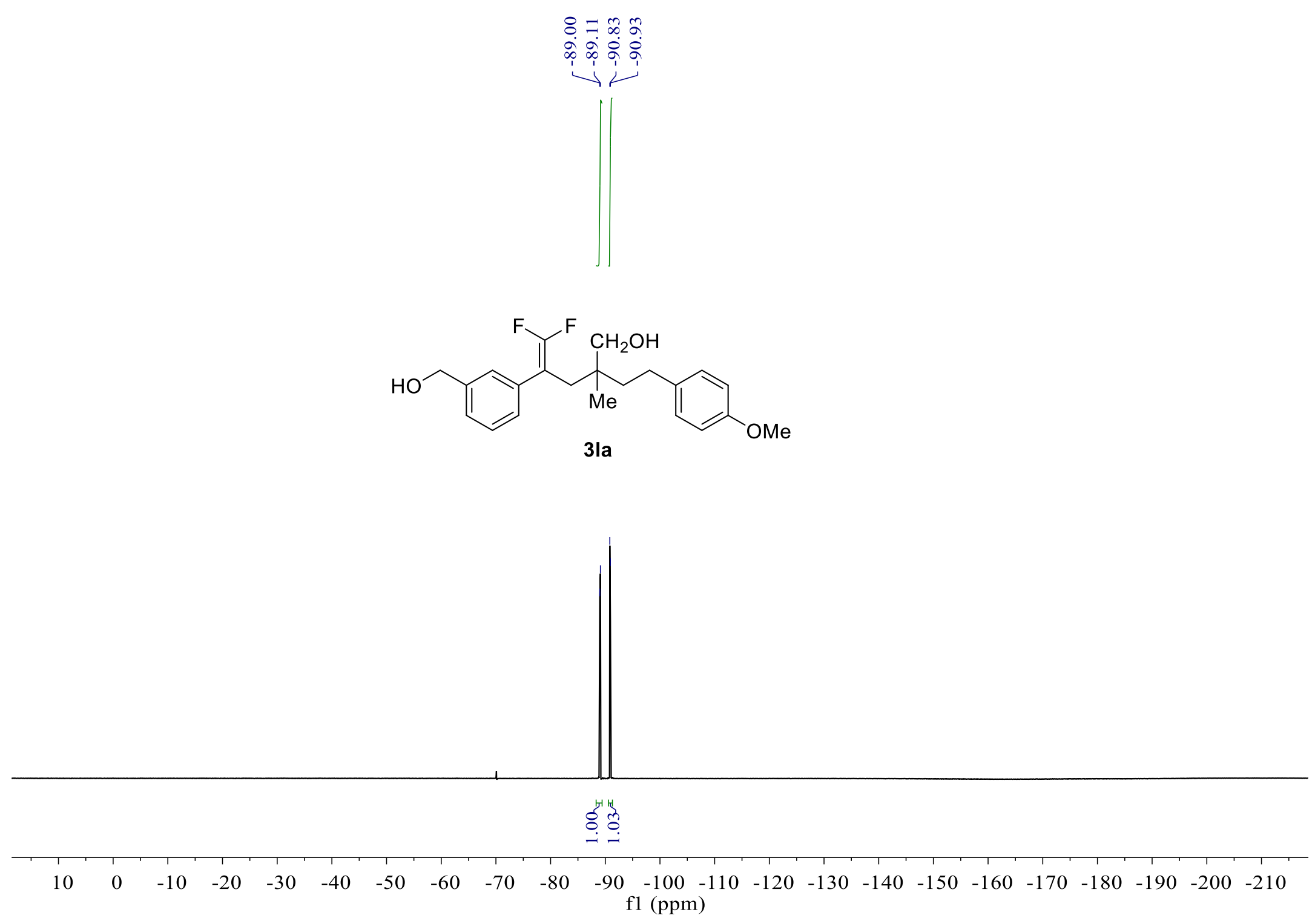

580 
${ }^{1} \mathrm{H}$ NMR-spectrum $\left(400 \mathrm{MHz}, \mathrm{CDCl}_{3}\right)$ of $\mathbf{3 m a}$

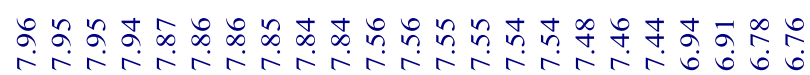

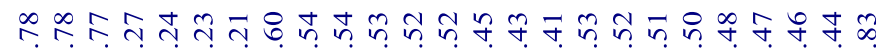

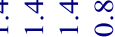

1 ,
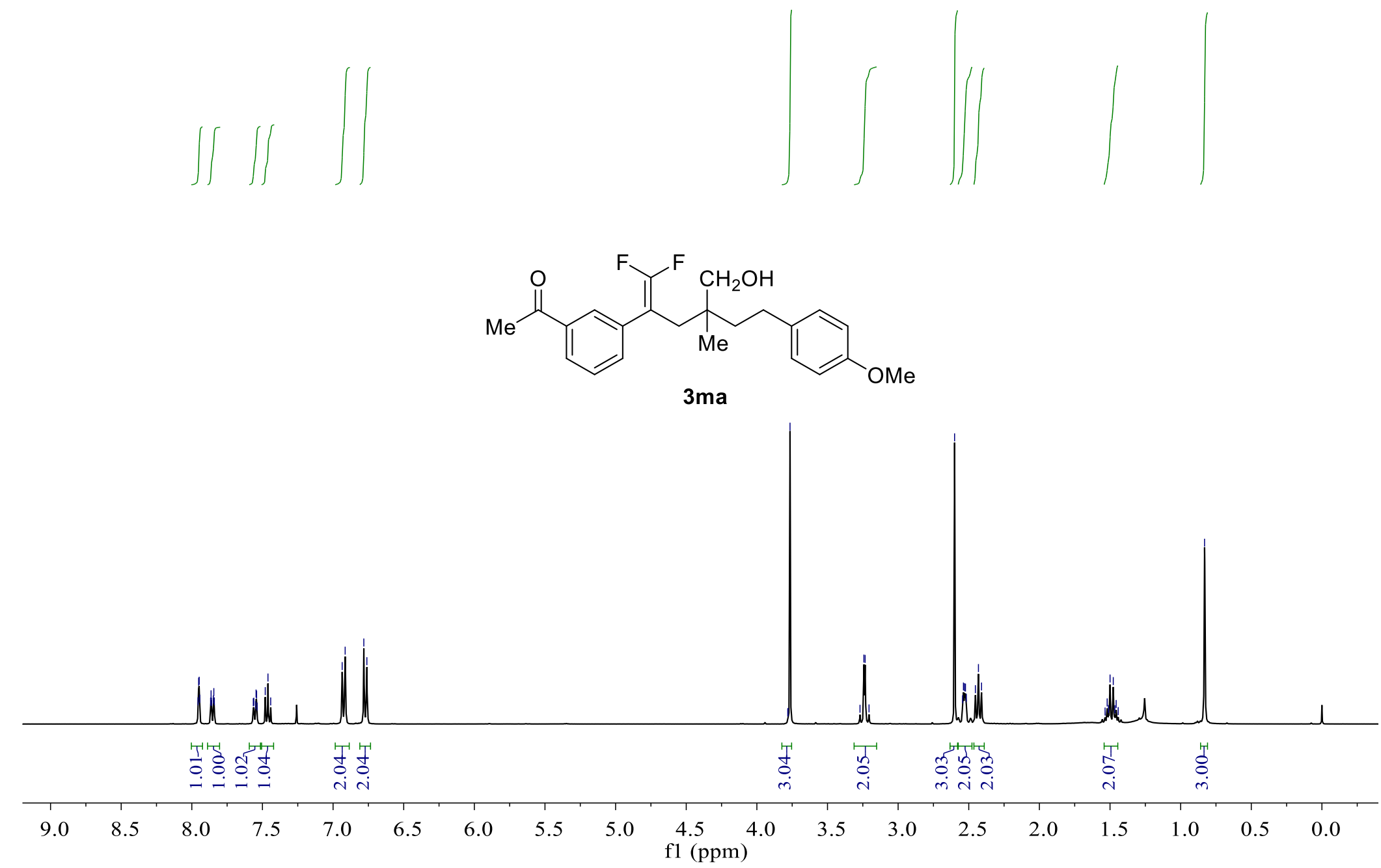

581 
${ }^{13} \mathrm{C}$ NMR-spectrum (101 MHz, $\mathrm{CDCl}_{3}$ ) of $\mathbf{3 m a}$
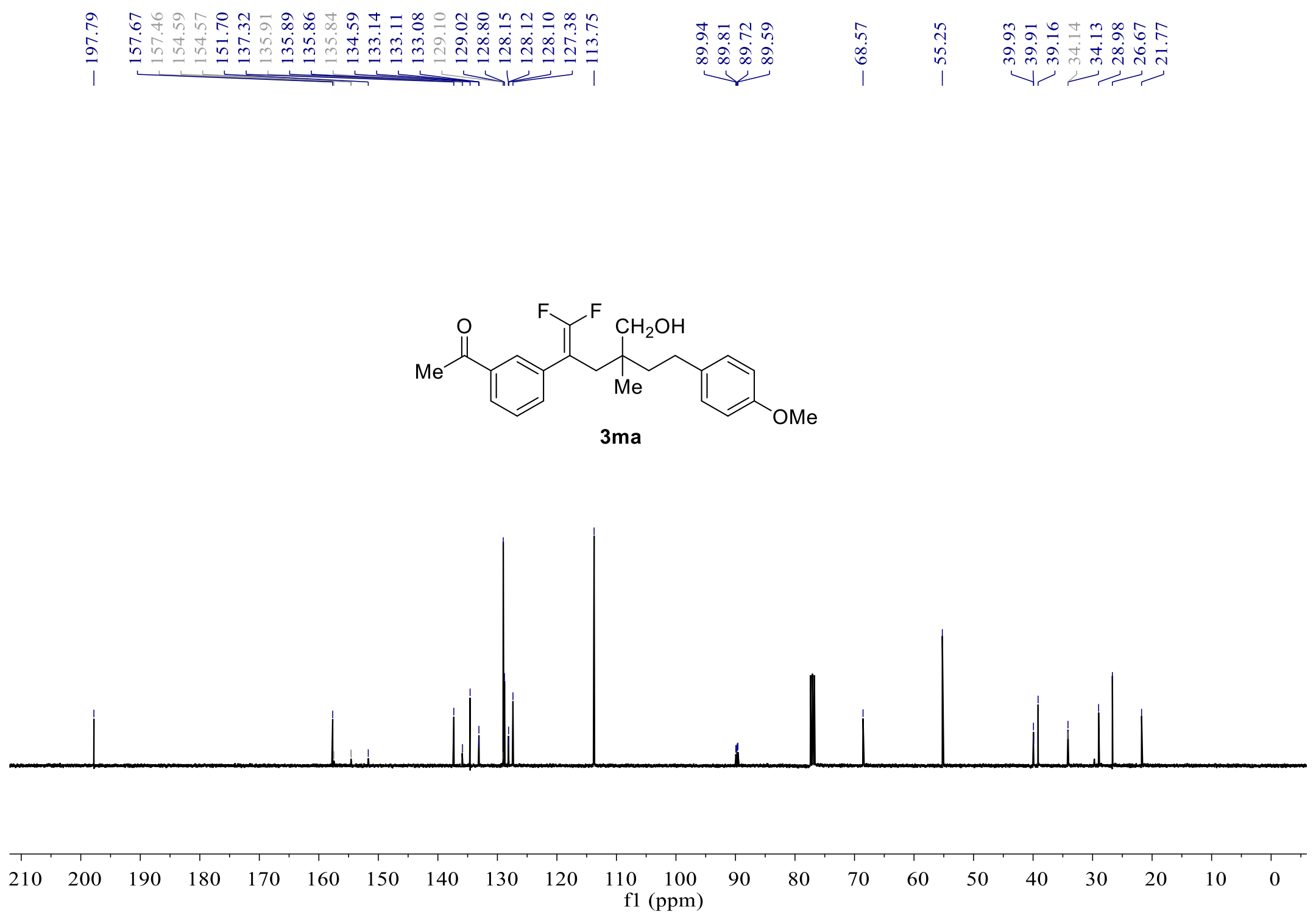

582 
${ }^{19} \mathrm{~F}$ NMR-spectrum $\left(376 \mathrm{MHz}, \mathrm{CDCl}_{3}\right)$ of $\mathbf{3 m a}$
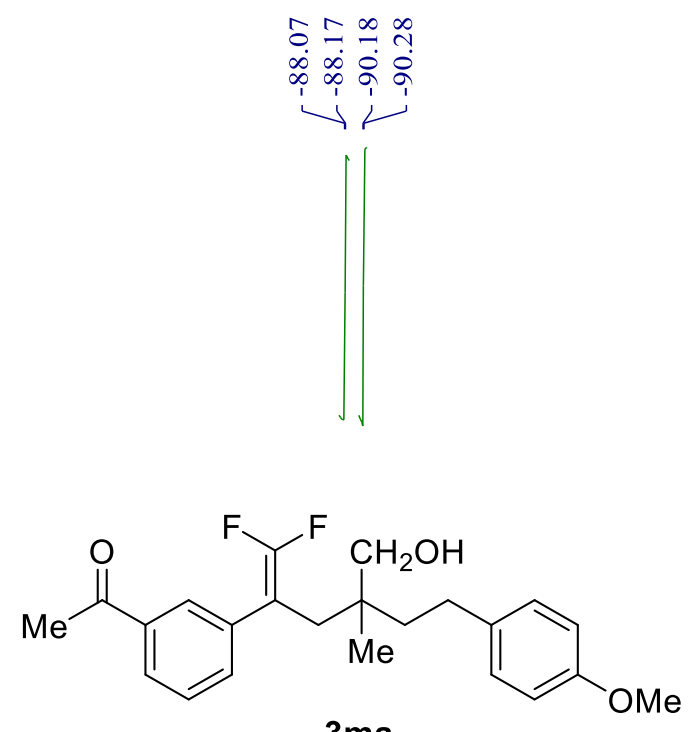

$3 \mathrm{ma}$

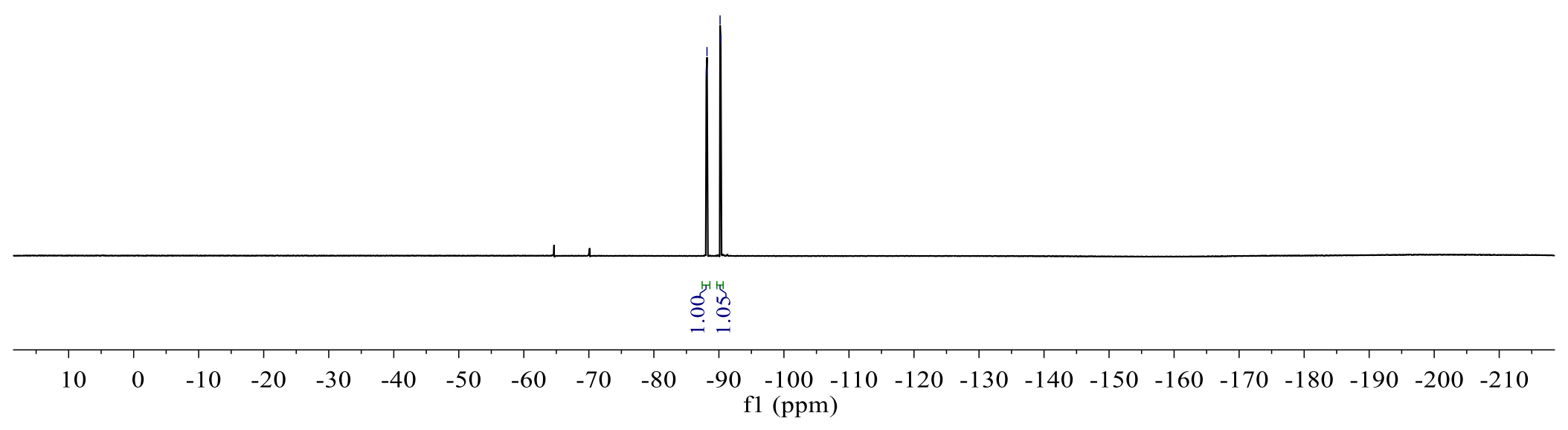




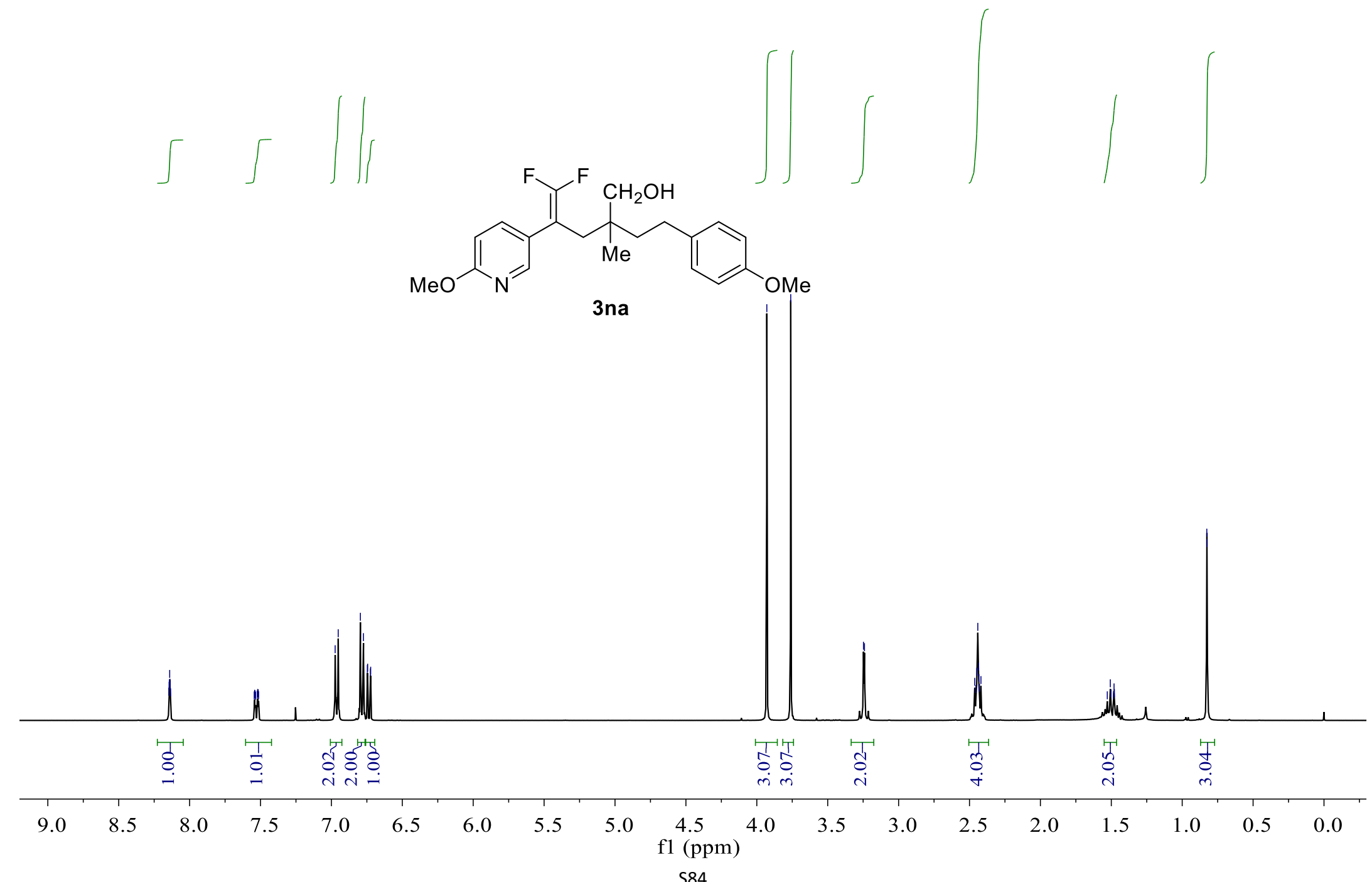


${ }^{13} \mathrm{C}$ NMR-spectrum (101 MHz, $\left.\mathrm{CDCl}_{3}\right)$ of 3na

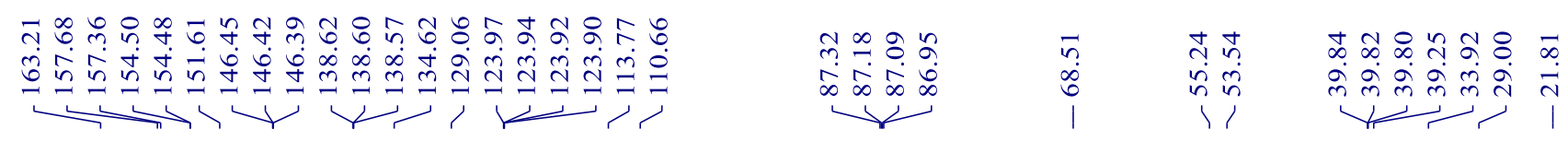
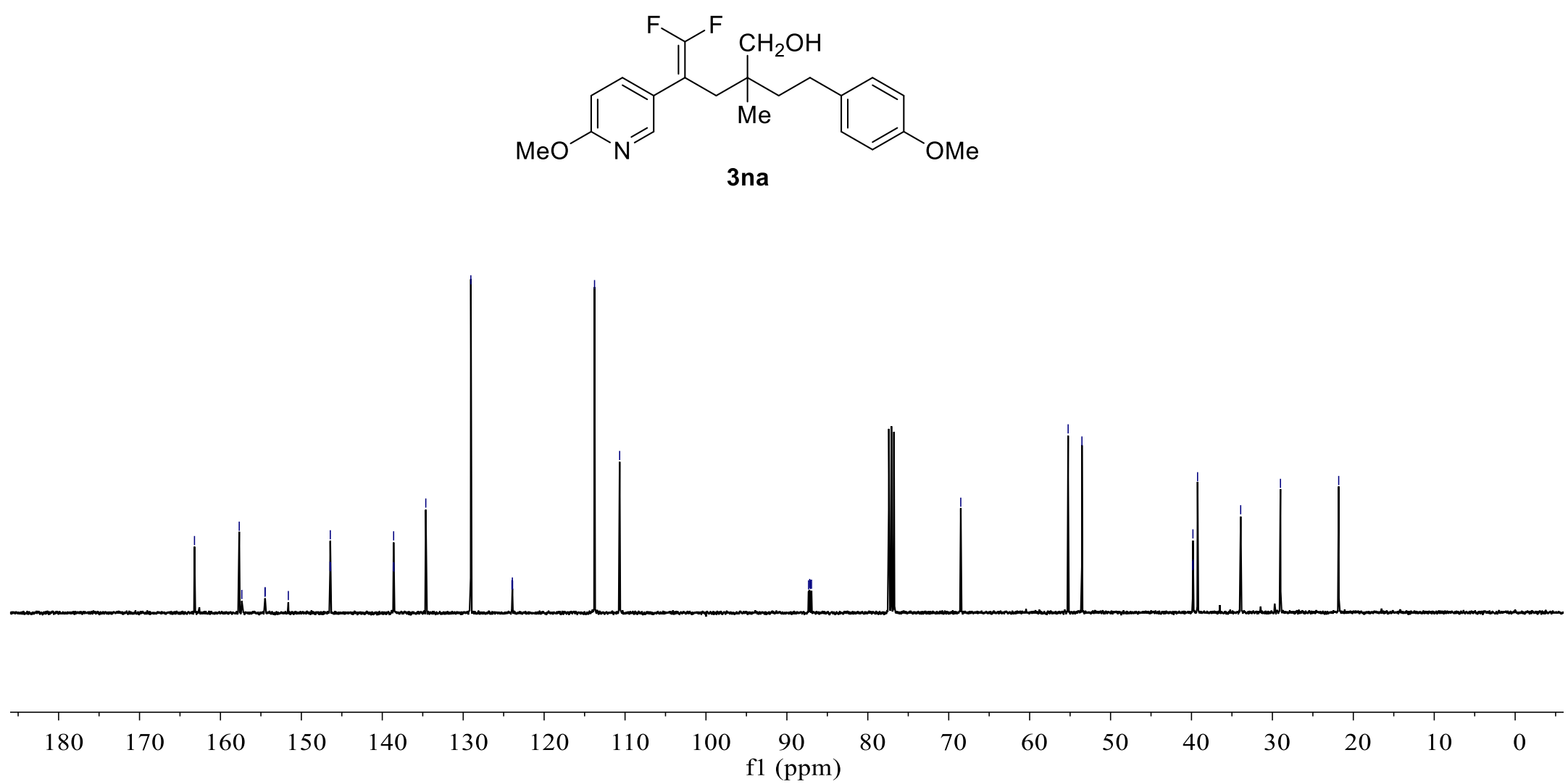

S85 
${ }^{19} \mathrm{~F}$ NMR-spectrum $\left(376 \mathrm{MHz}, \mathrm{CDCl}_{3}\right.$ ) of 3na
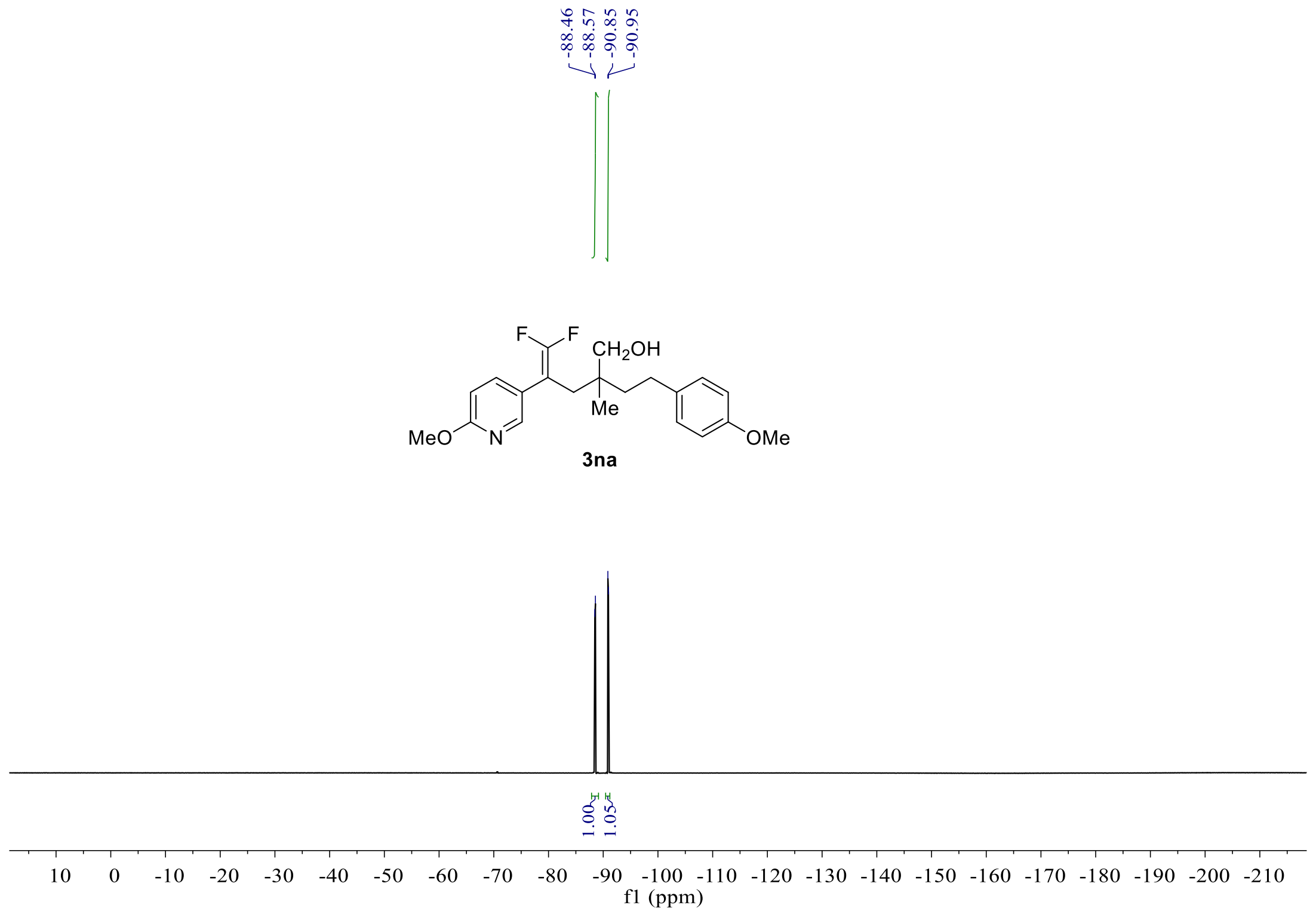

S86 
$=\frac{0}{\infty}$

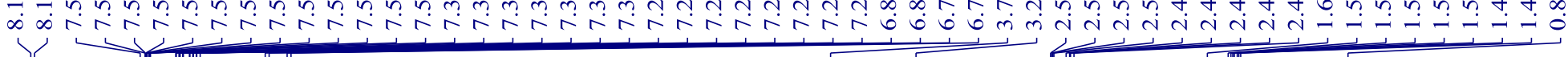

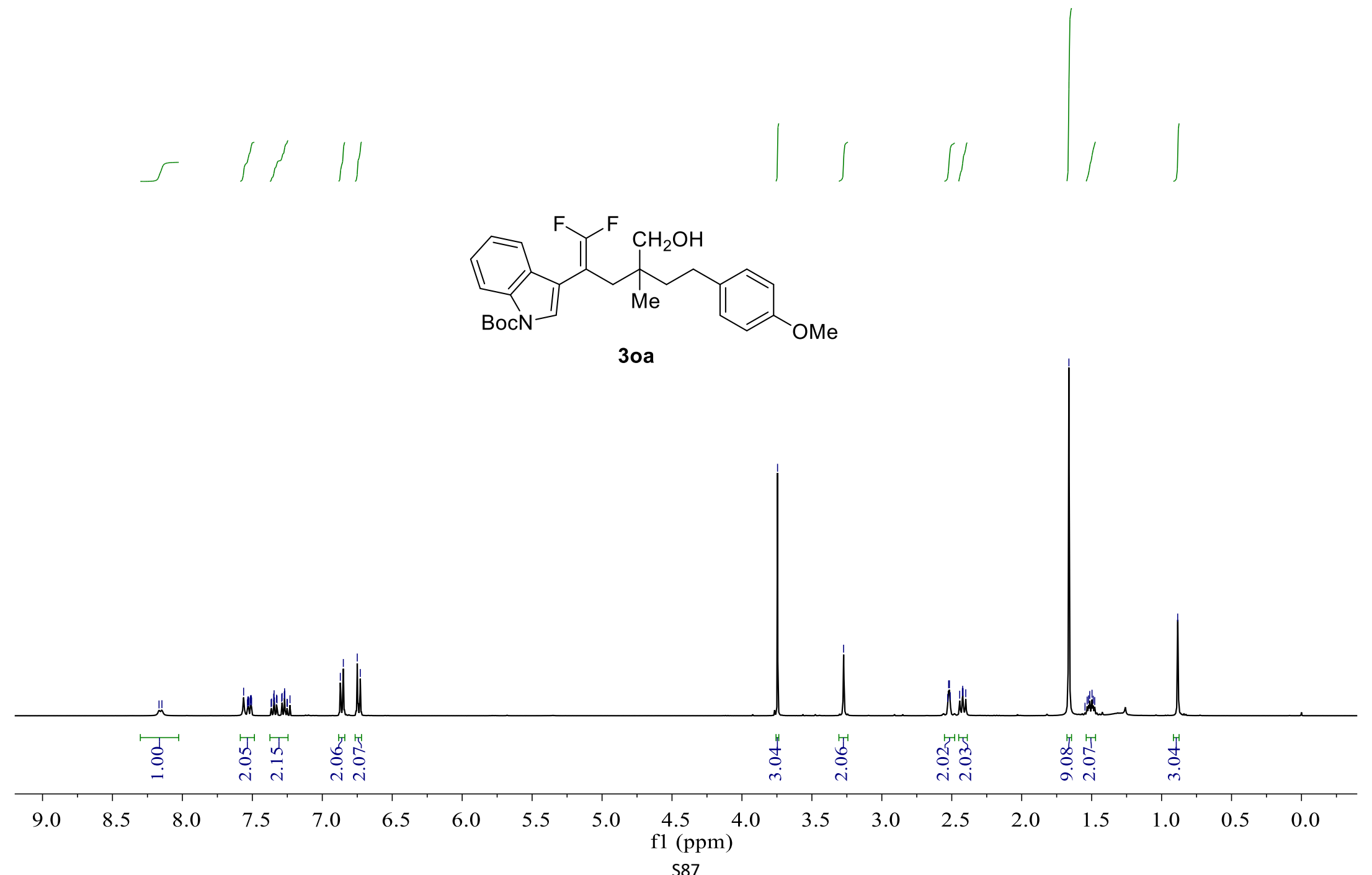

s87 
${ }^{13} \mathrm{C}$ NMR-spectrum $\left(101 \mathrm{MHz}, \mathrm{CDCl}_{3}\right)$ of $\mathbf{3 o a}$
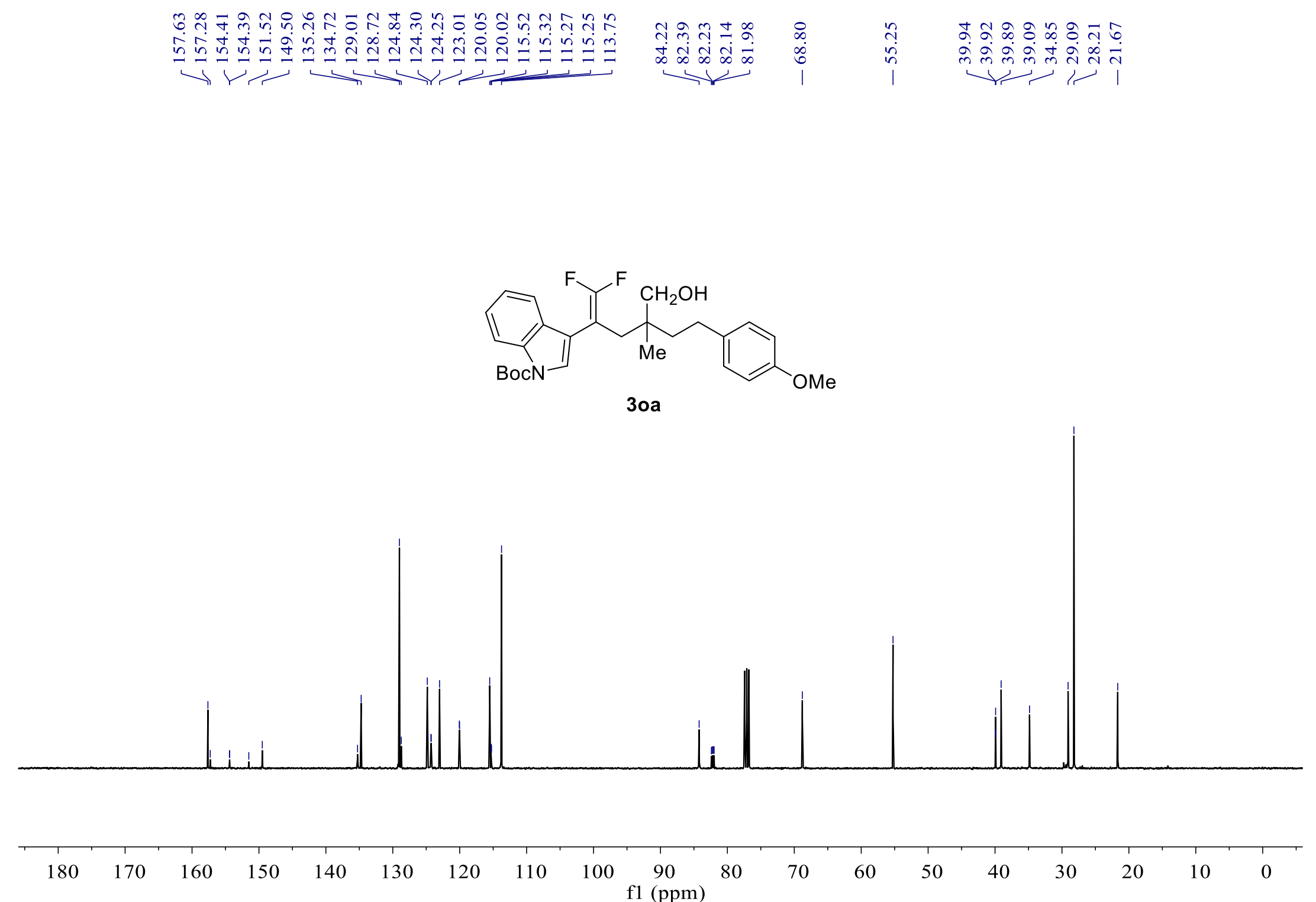

$\mathrm{S} 88$ 
${ }^{19} \mathrm{~F} \mathrm{NMR}$-spectrum $\left(376 \mathrm{MHz}, \mathrm{CDCl}_{3}\right.$ ) of $\mathbf{3 o a}$
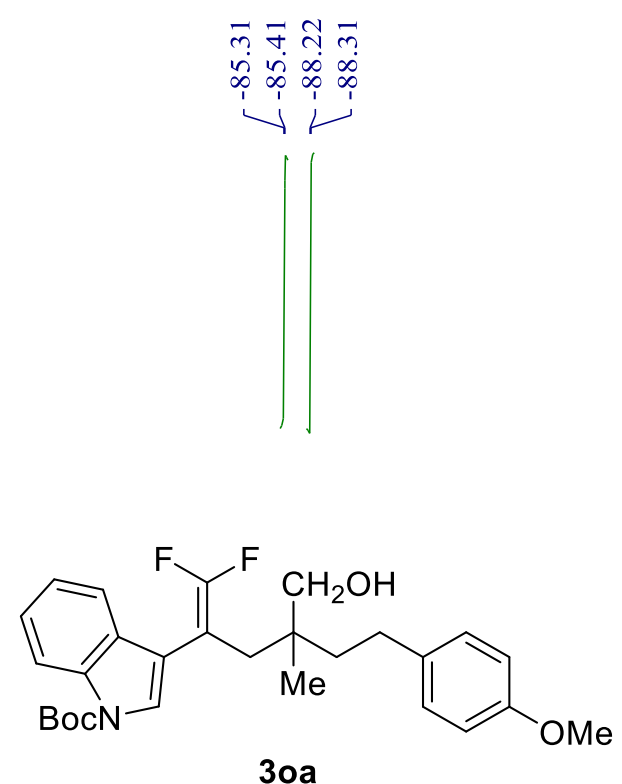

$30 a$

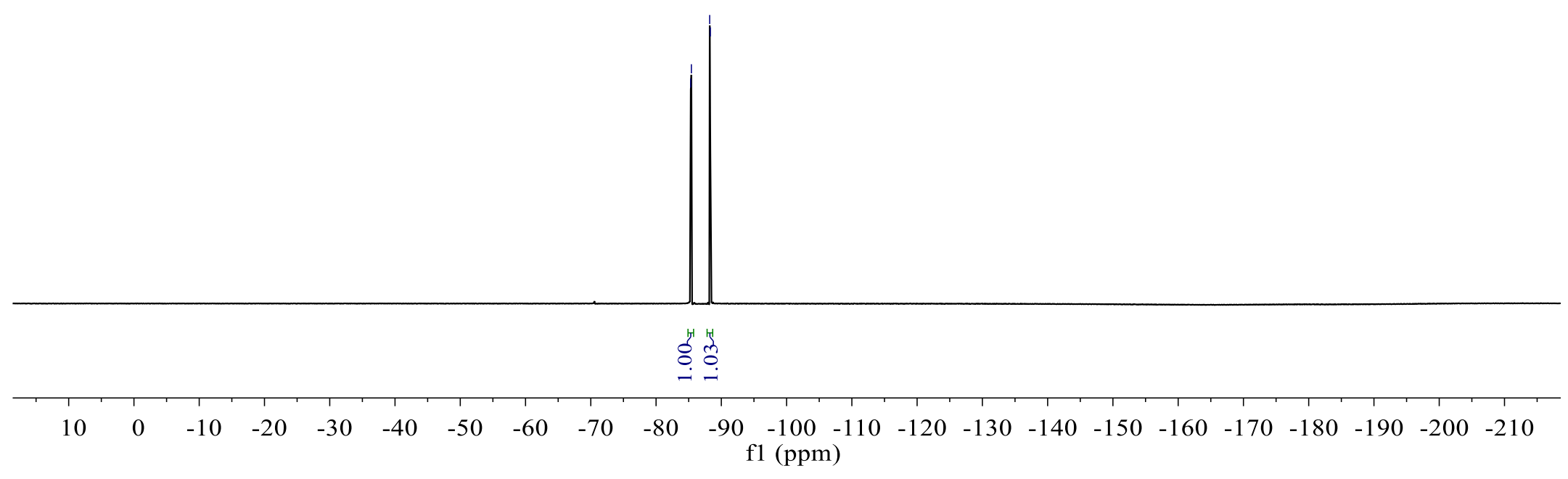

589 
${ }^{1} \mathrm{H}$ NMR-spectrum $\left(400 \mathrm{MHz}, \mathrm{CDCl}_{3}\right)$ of $\mathbf{3 p a}$

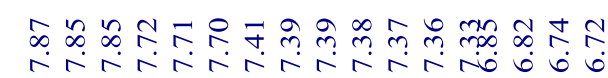

(1)

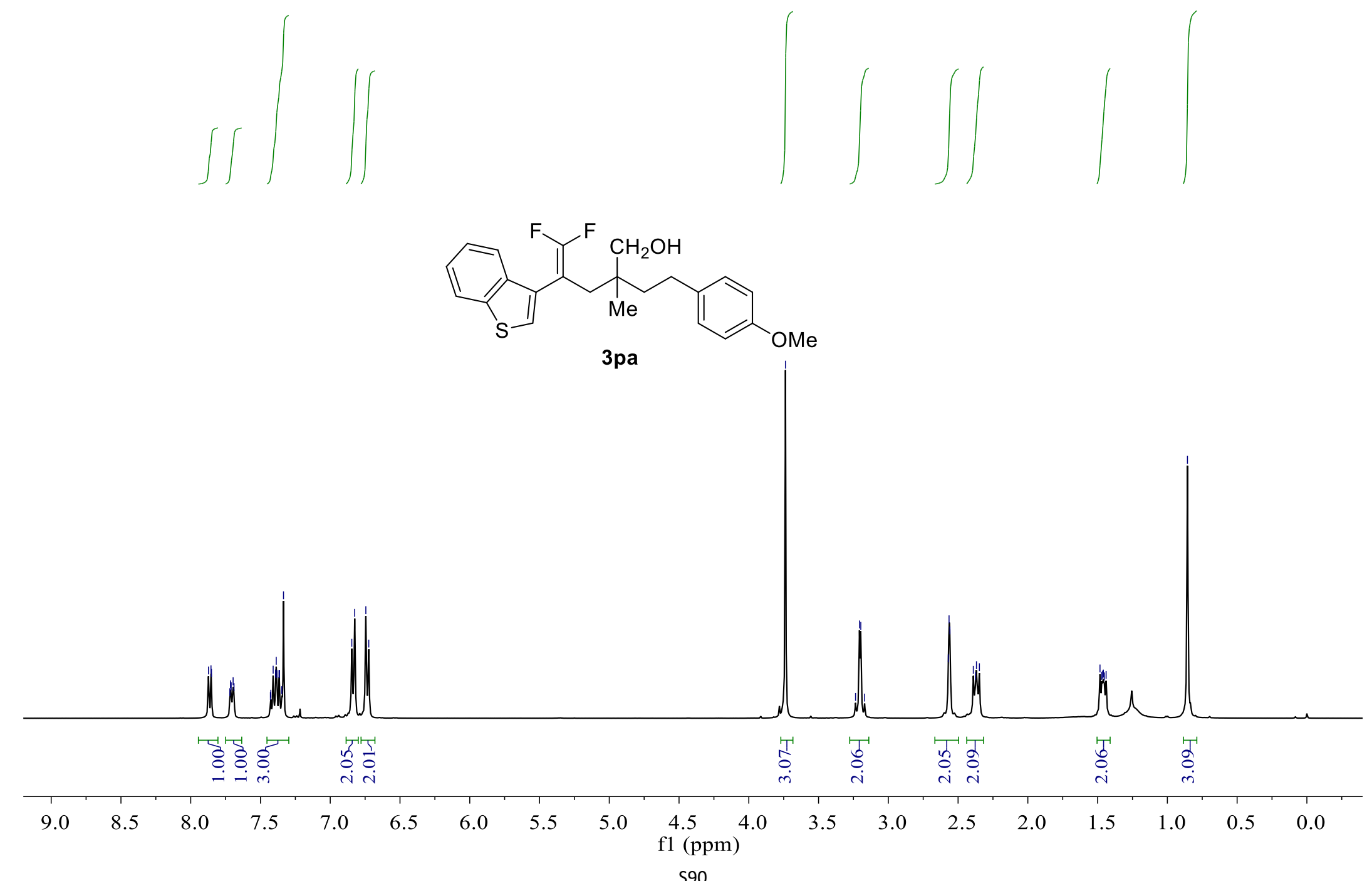

萑

s9o 
${ }^{13} \mathrm{C}$ NMR-spectrum $\left(101 \mathrm{MHz}, \mathrm{CDCl}_{3}\right)$ of 3pa
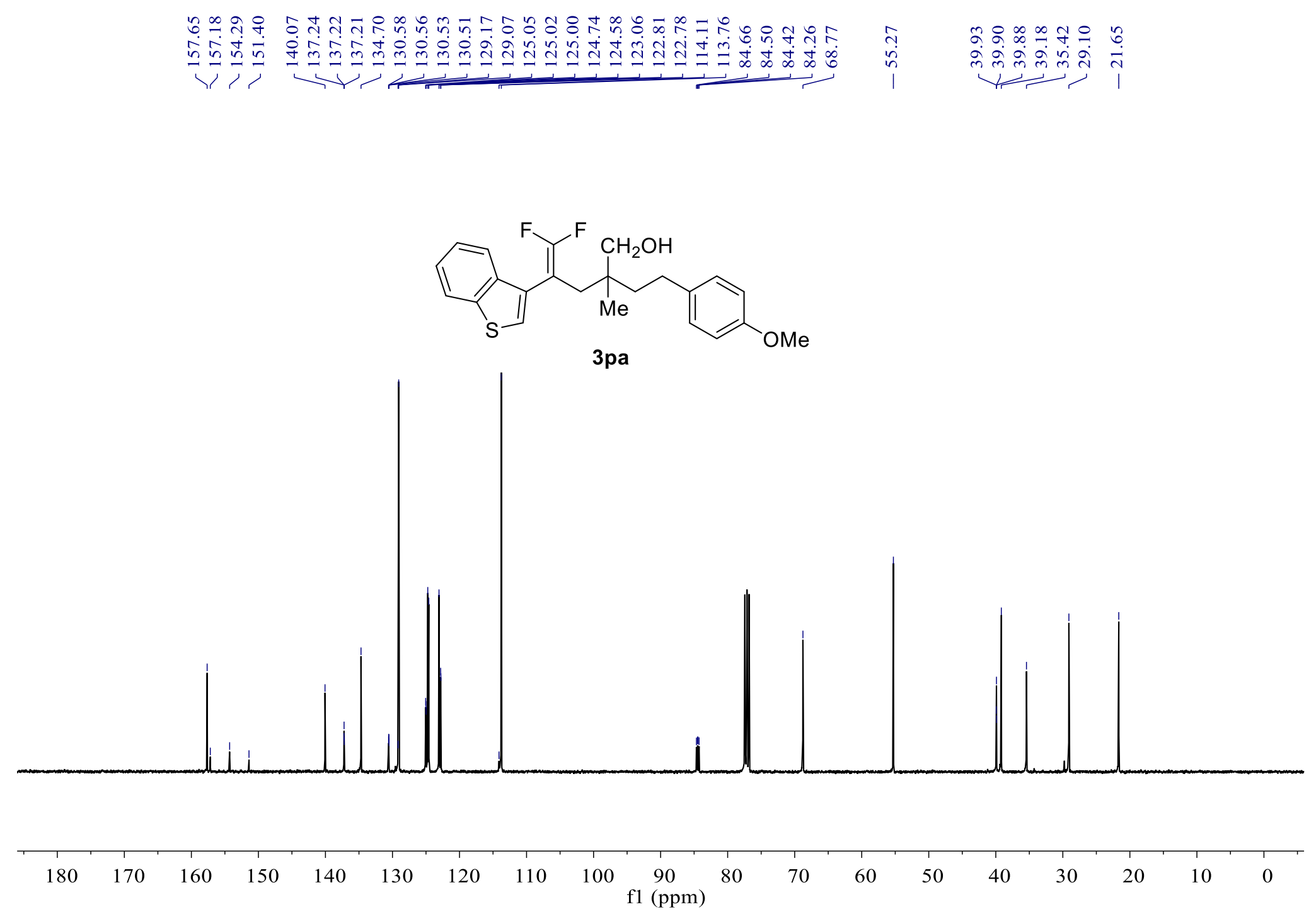

s91 
${ }^{19} \mathrm{~F}$ NMR-spectrum $\left(376 \mathrm{MHz}, \mathrm{CDCl}_{3}\right.$ ) of $\mathbf{3 p a}$
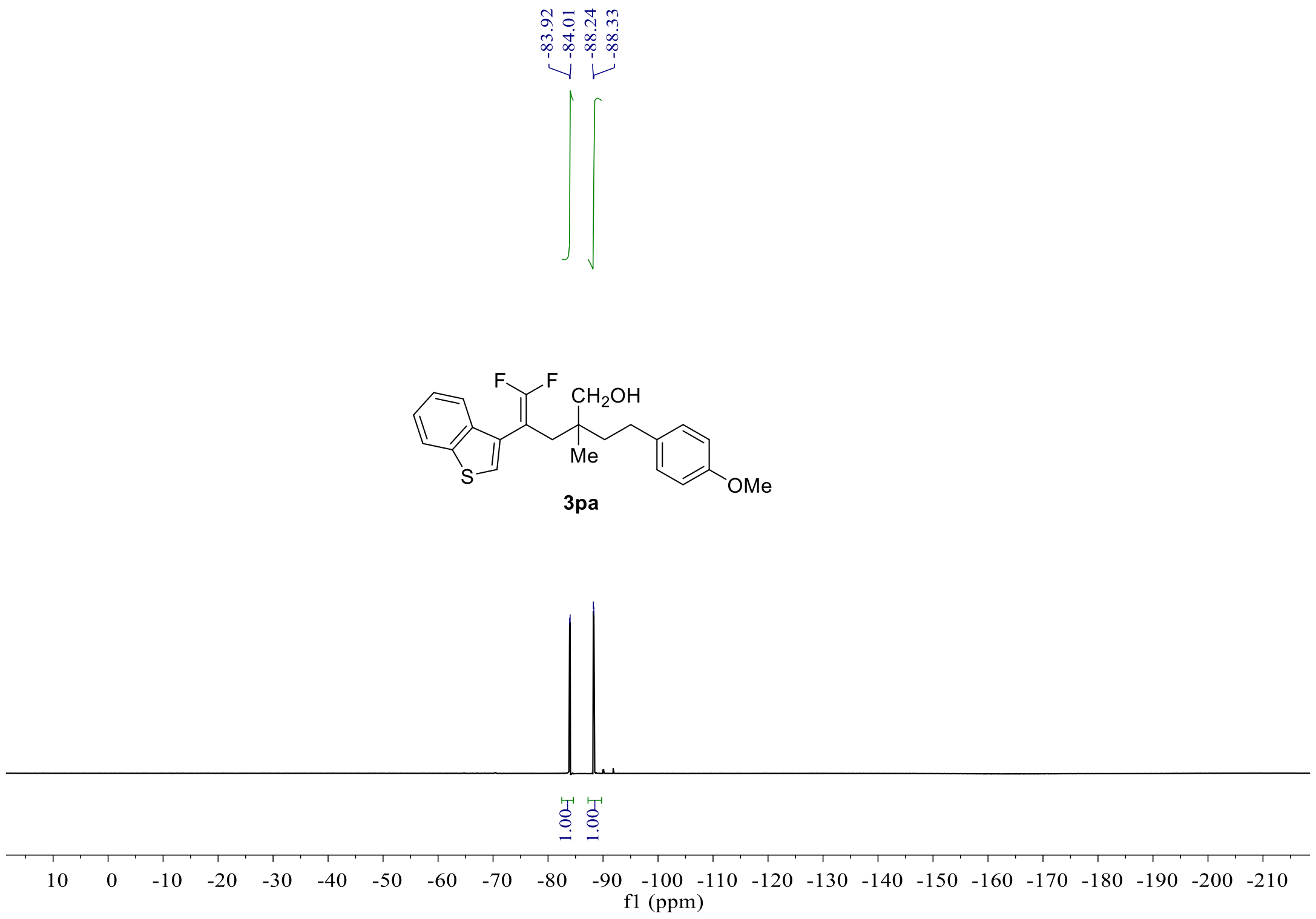


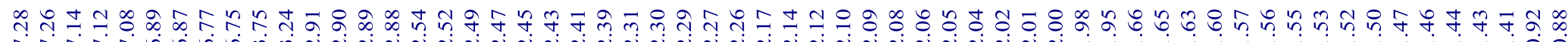

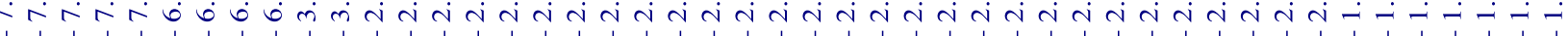

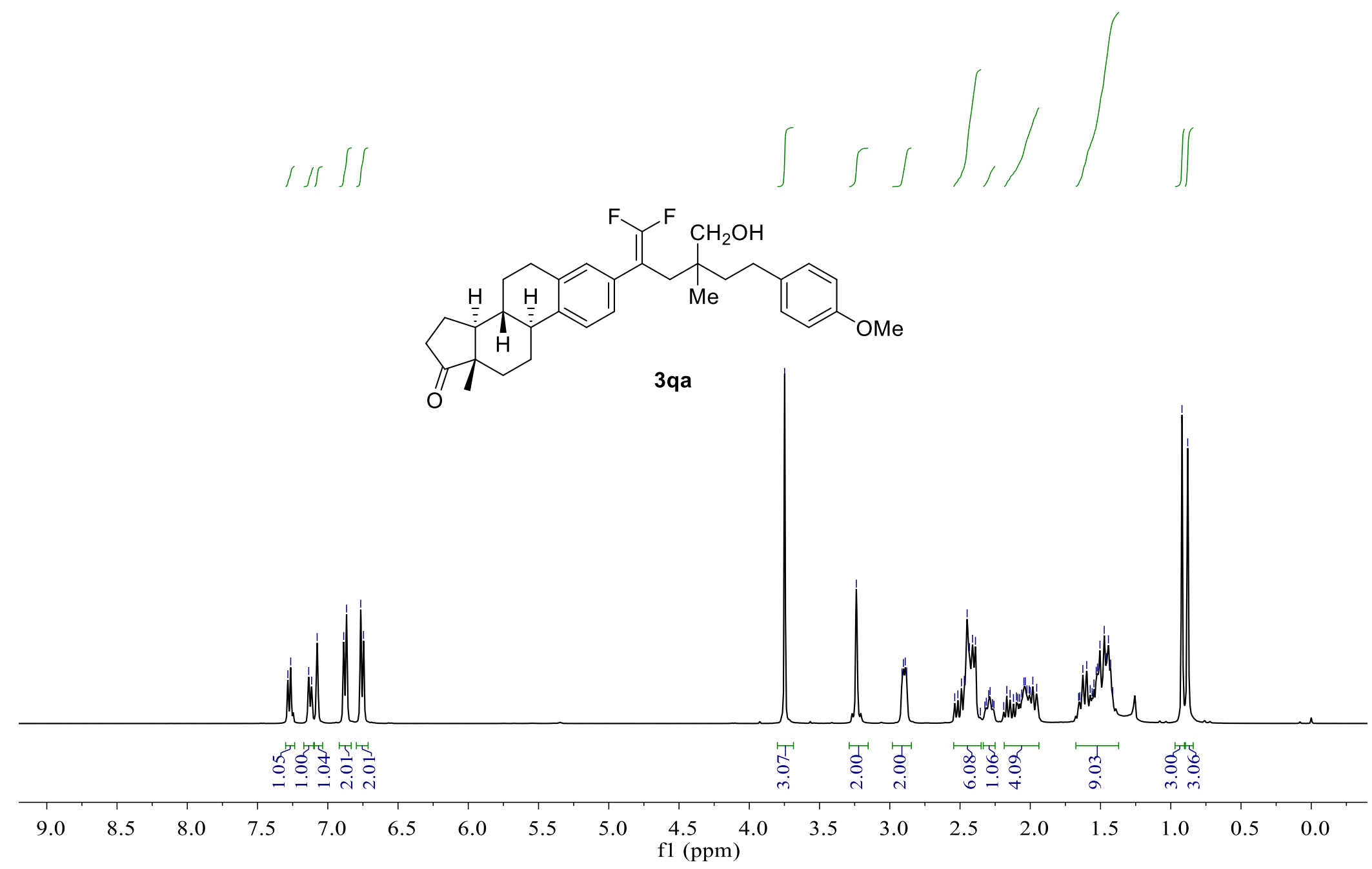


${ }^{13} \mathrm{C}$ NMR-spectrum $\left(101 \mathrm{MHz}, \mathrm{CDCl}_{3}\right)$ of $\mathbf{3 q a}$
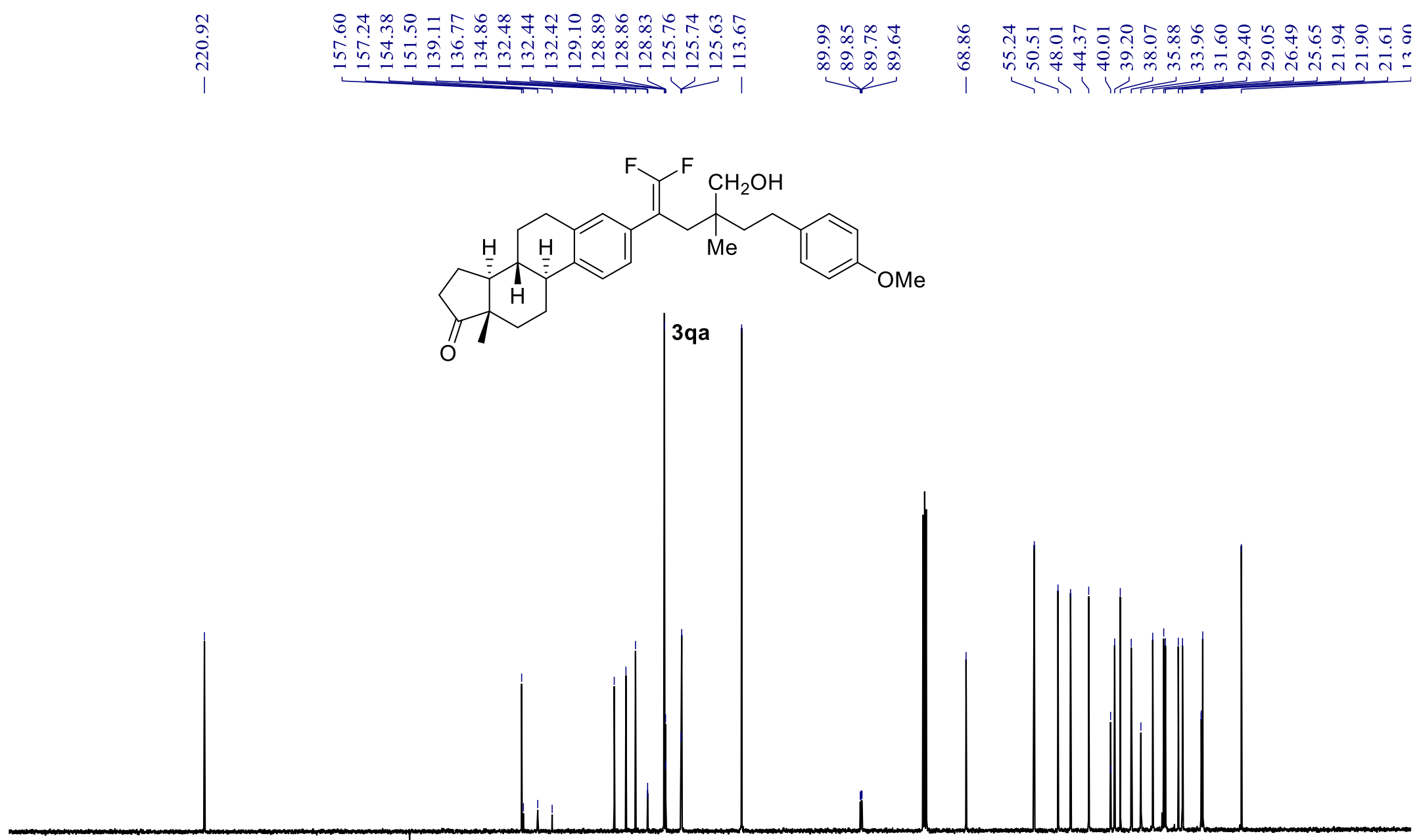

$\begin{array}{lllllllllllllllllllllllllllll}60 & 250 & 240 & 230 & 220 & 210 & 200 & 190 & 180 & 170 & 160 & 150 & 140 & 130 & 120 & 110 & 100 & 90 & 80 & 70 & 60 & 50 & 40 & 30 & 20 & 10 & 0 & -10 & -\end{array}$ f1 (ppm) 
${ }^{19} \mathrm{~F}$ NMR-spectrum $\left(376 \mathrm{MHz}, \mathrm{CDCl}_{3}\right)$ of $\mathbf{3 q a}$

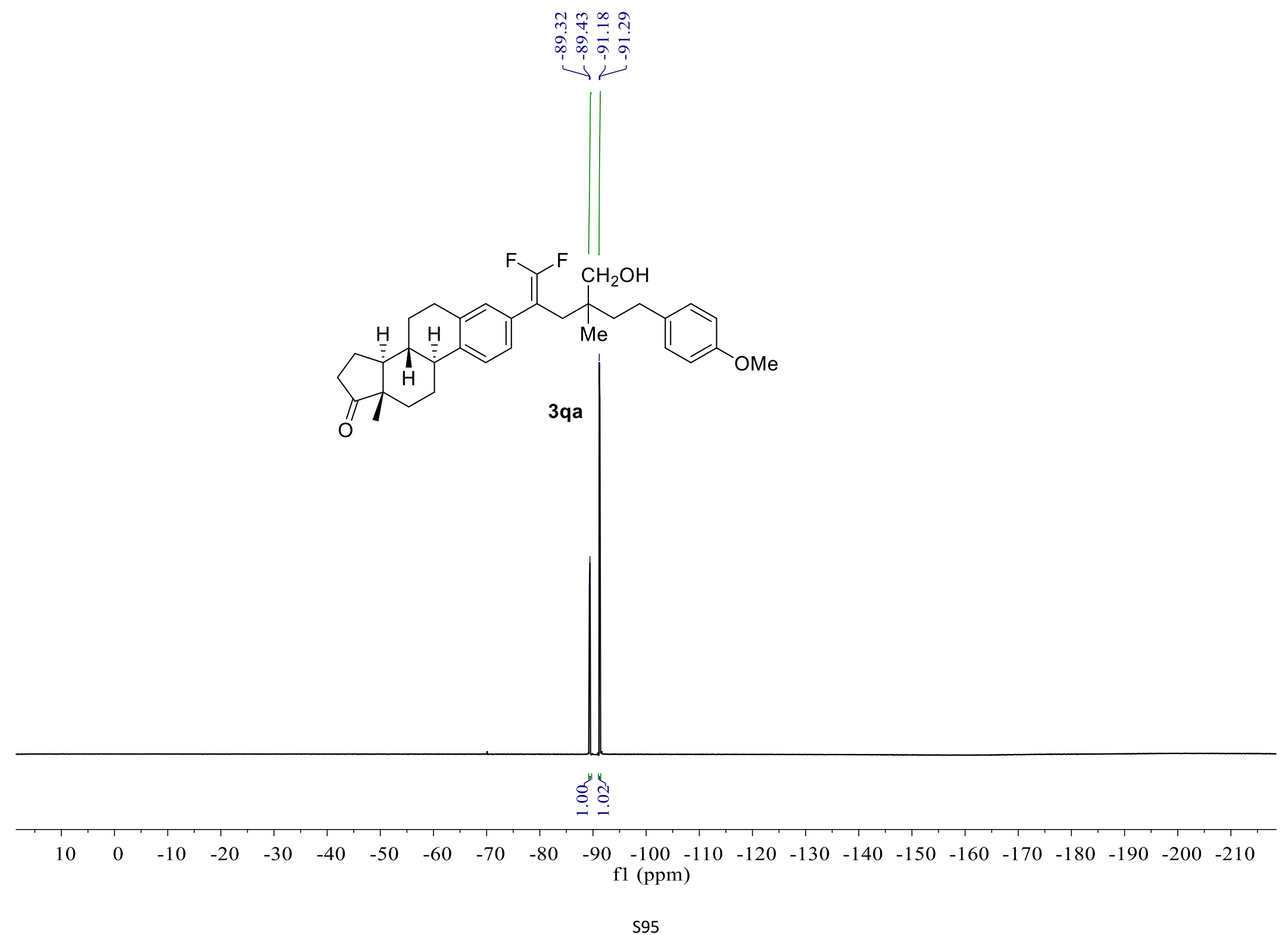


${ }^{1} \mathrm{H}$ NMR-spectrum (400 MHz, $\mathrm{CDCl}_{3}$ ) of $\mathbf{3 a b}$

în

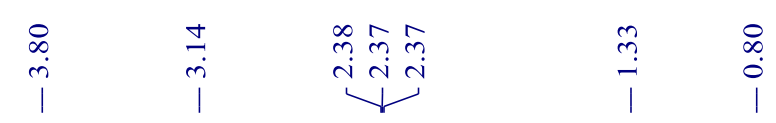

$\int \Gamma$
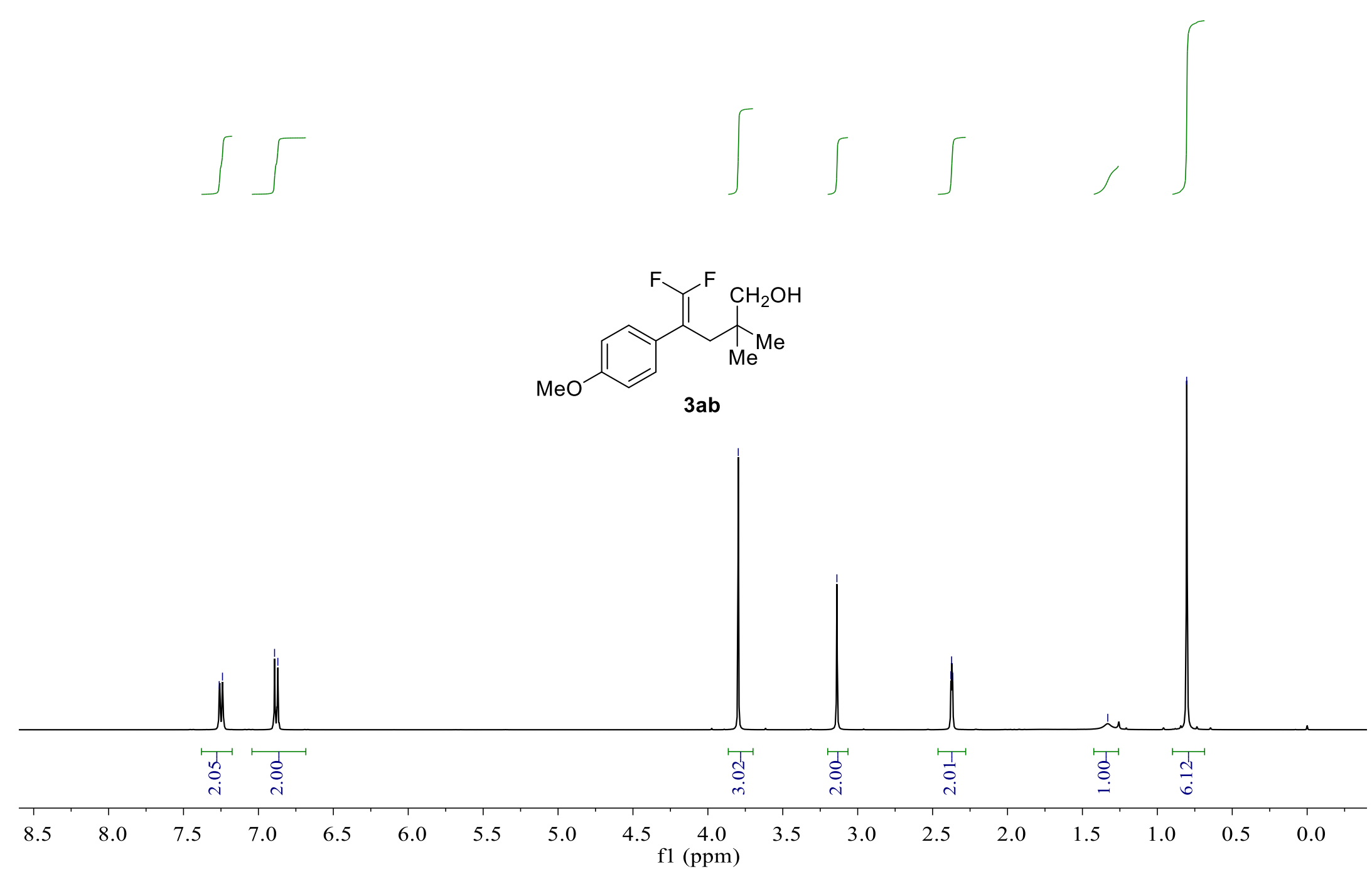
${ }^{13} \mathrm{C}$ NMR-spectrum $\left(101 \mathrm{MHz}, \mathrm{CDCl}_{3}\right)$ of $\mathbf{3 a b}$

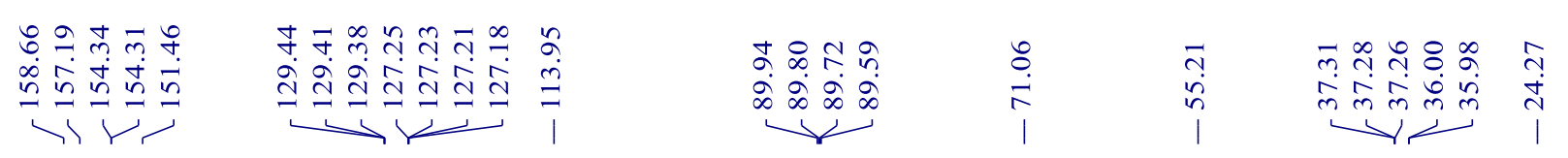

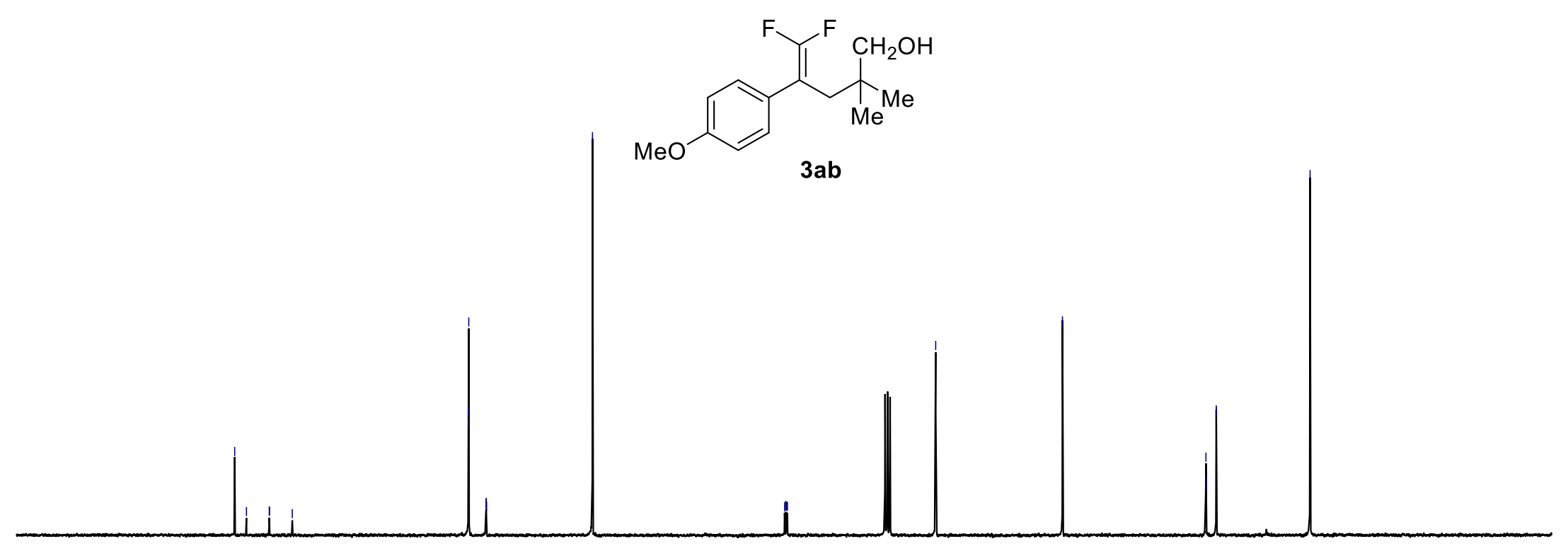

$180 \quad 170 \quad 160$

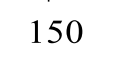

140

130

120

110

$\begin{array}{ll}90 & 90\end{array}$

70

60

40

30

$20 \quad 10 \quad 0$

S97 
${ }^{19} \mathrm{~F}$ NMR-spectrum (376 MHz, $\mathrm{CDCl}_{3}$ ) of $\mathbf{3 a b}$

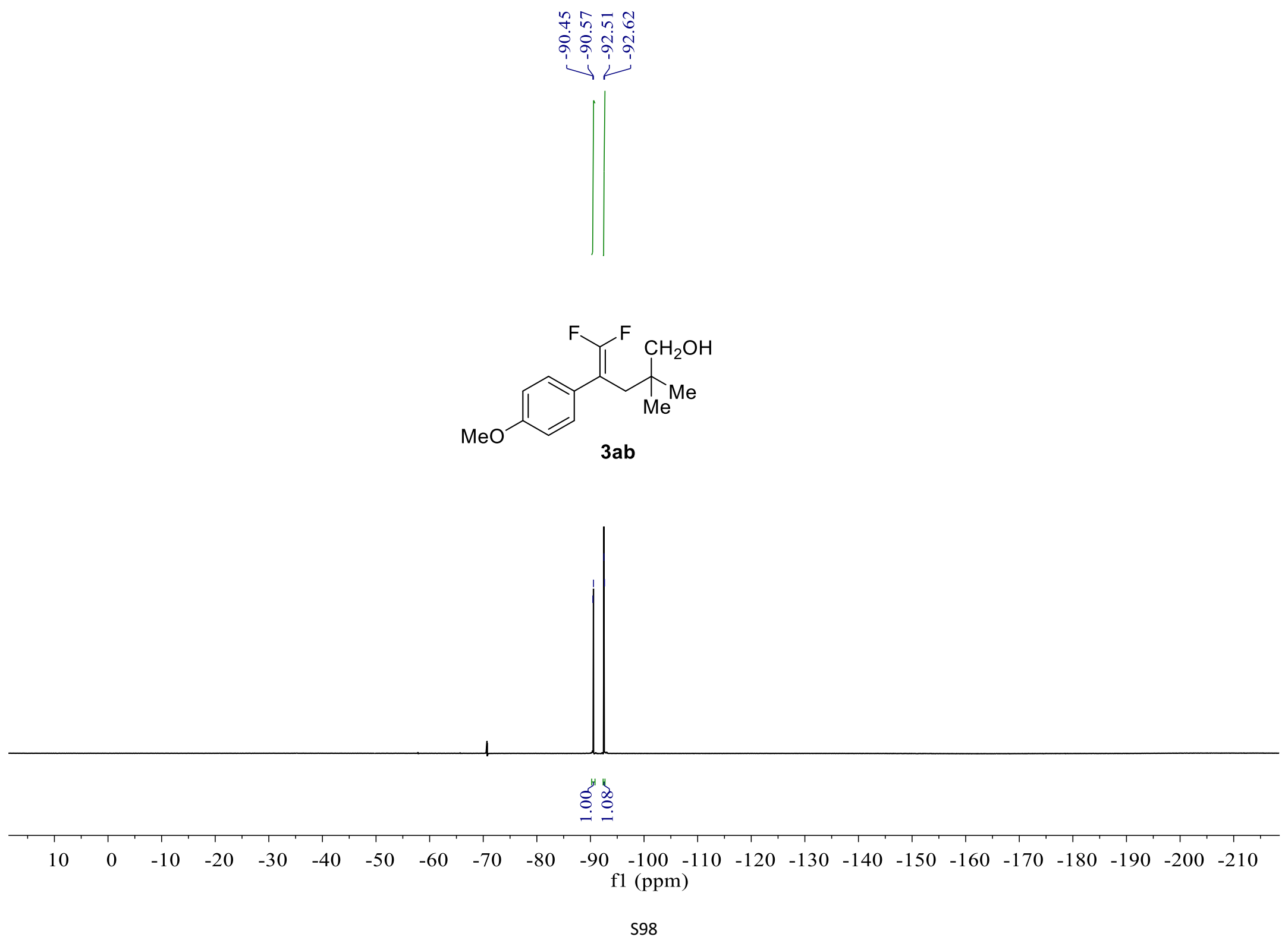


${ }^{1} \mathrm{H}$ NMR-spectrum $\left(400 \mathrm{MHz}, \mathrm{CDCl}_{3}\right)$ of $\mathbf{3 a c}$

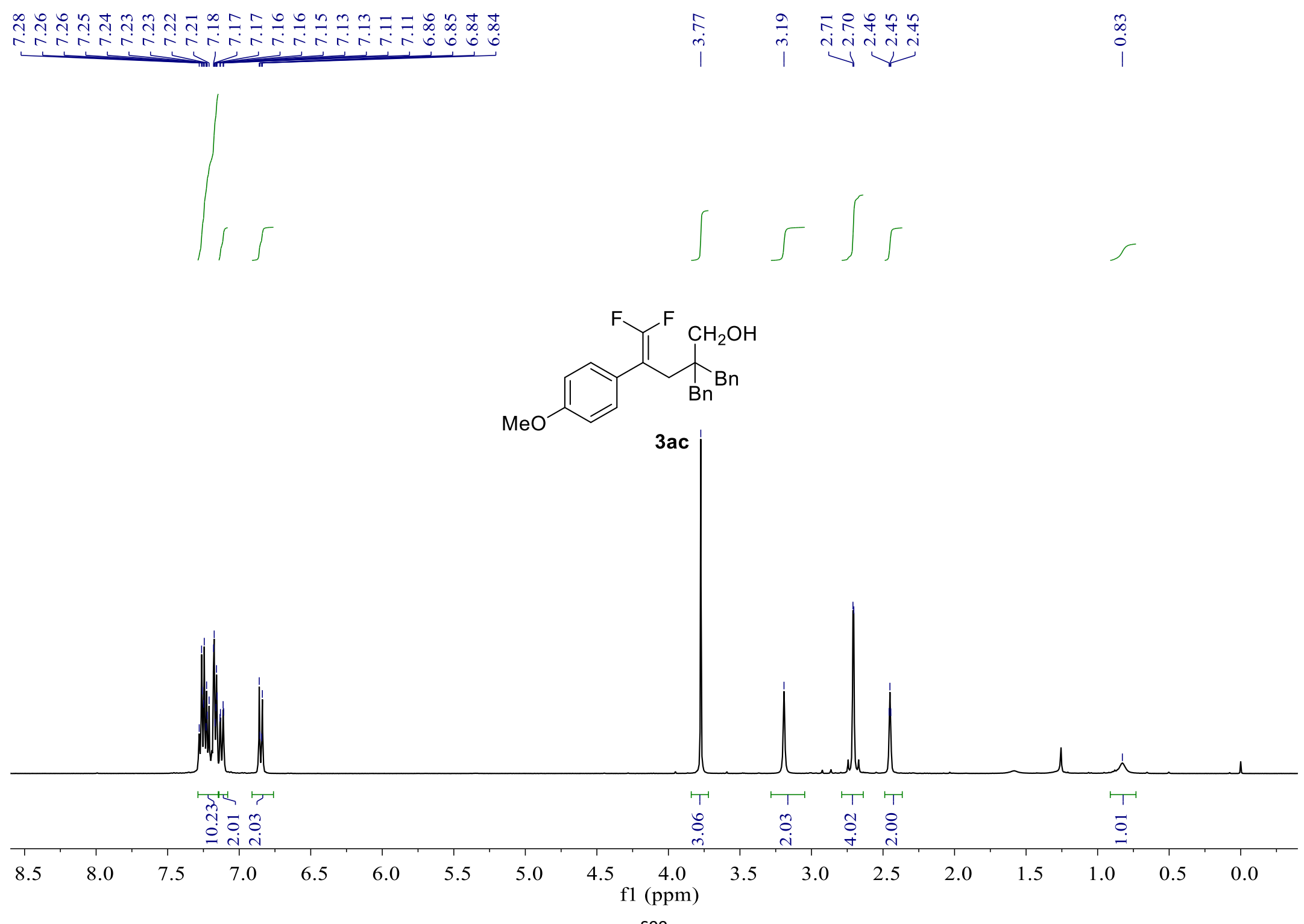

s99 
${ }^{13} \mathrm{C}$ NMR-spectrum $\left(101 \mathrm{MHz}, \mathrm{CDCl}_{3}\right)$ of $\mathbf{3 a c}$

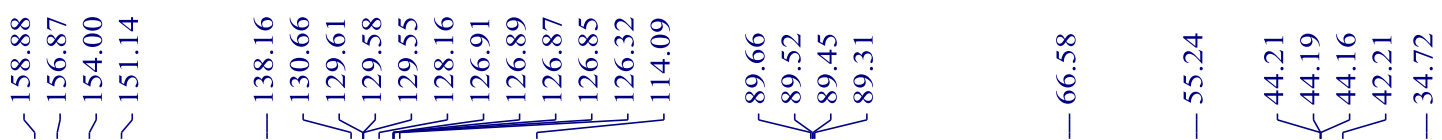
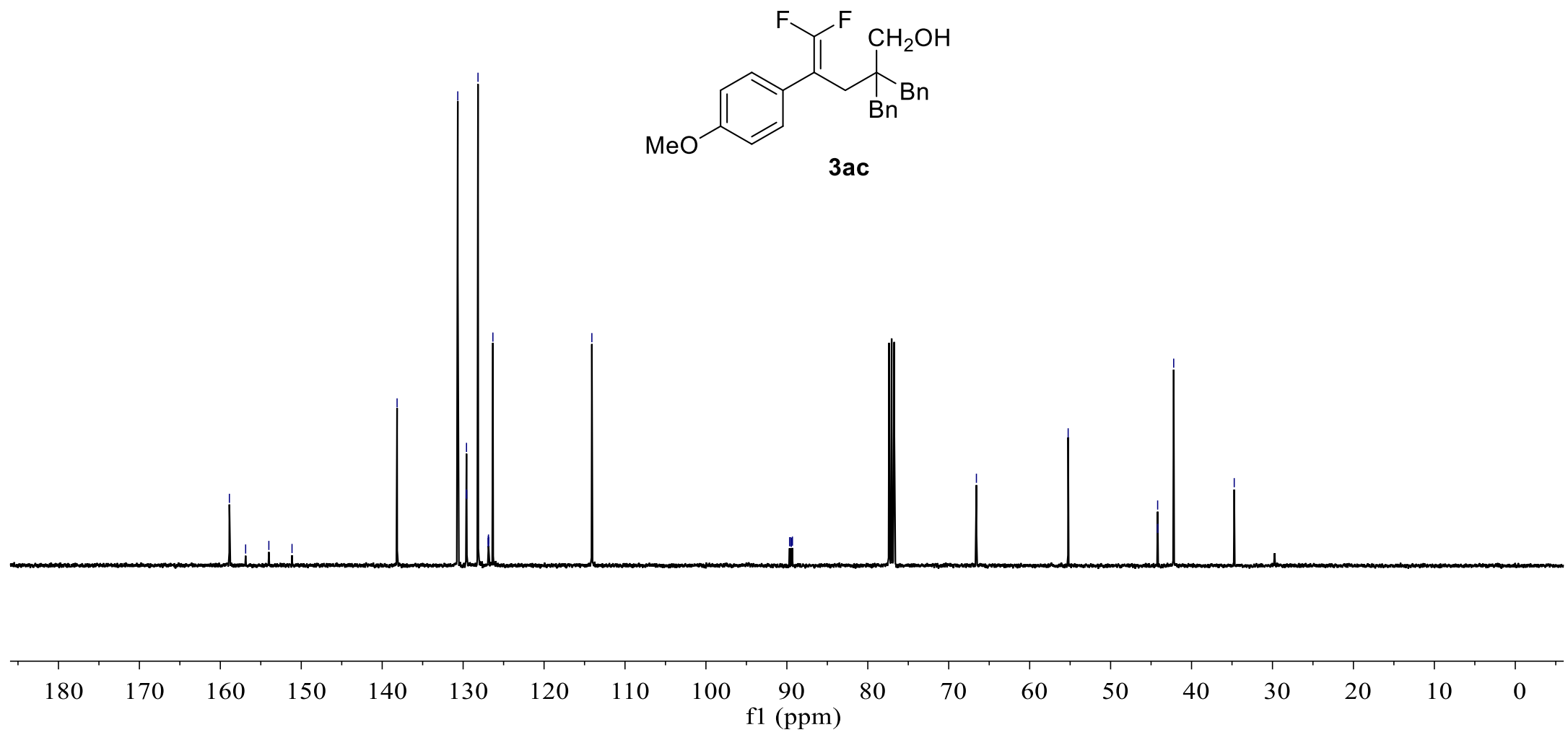
${ }^{19} \mathrm{~F}$ NMR-spectrum $\left(376 \mathrm{MHz}, \mathrm{CDCl}_{3}\right.$ ) of $\mathbf{3 a c}$
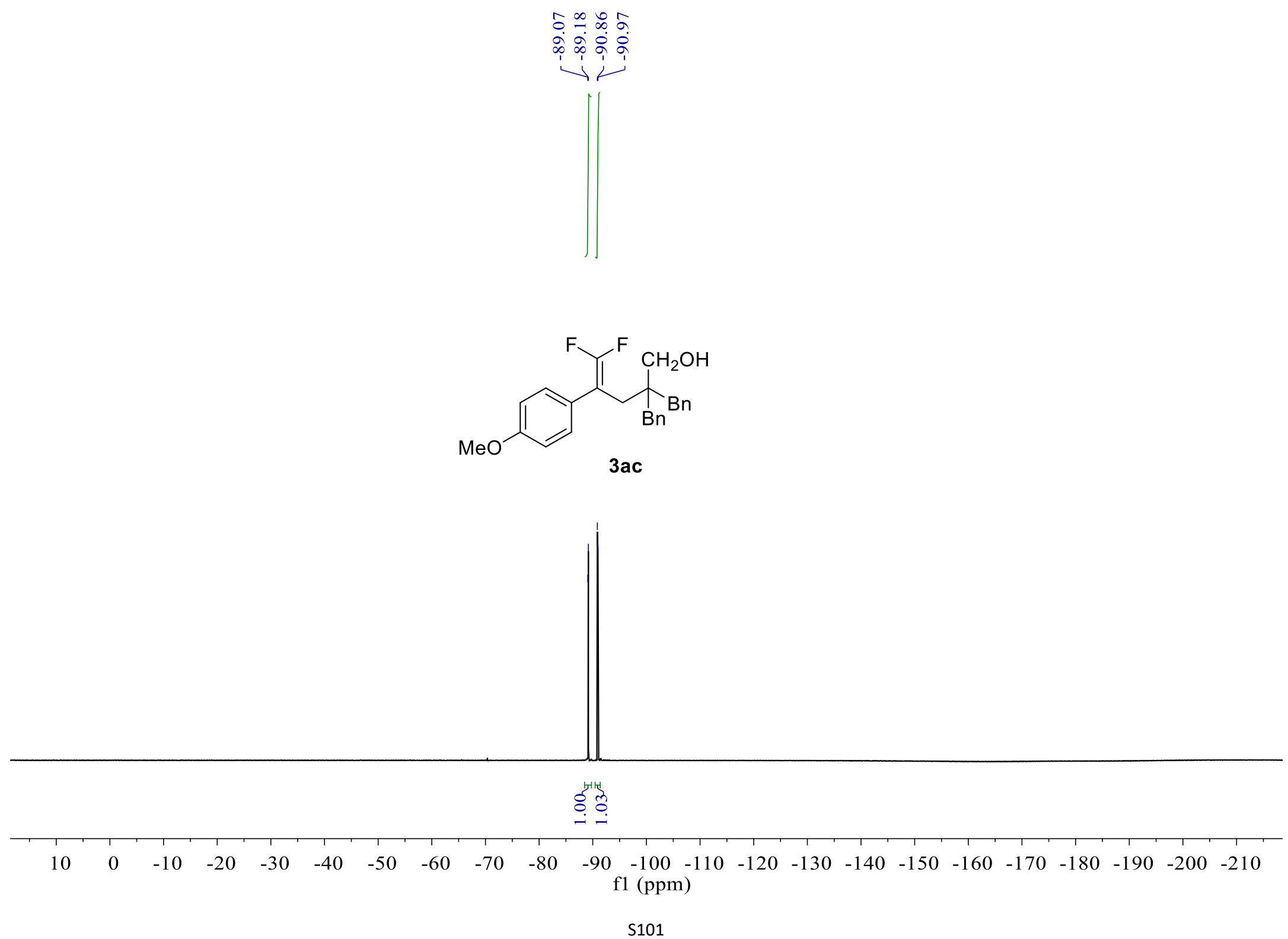
${ }^{1} \mathrm{H}$ NMR-spectrum (400 MHz, $\mathrm{CDCl}_{3}$ ) of $\mathbf{3 a d}$

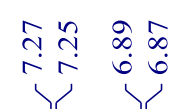

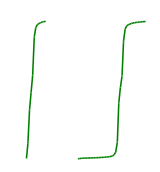

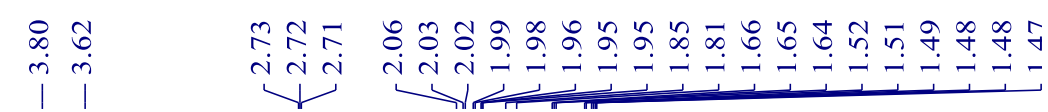

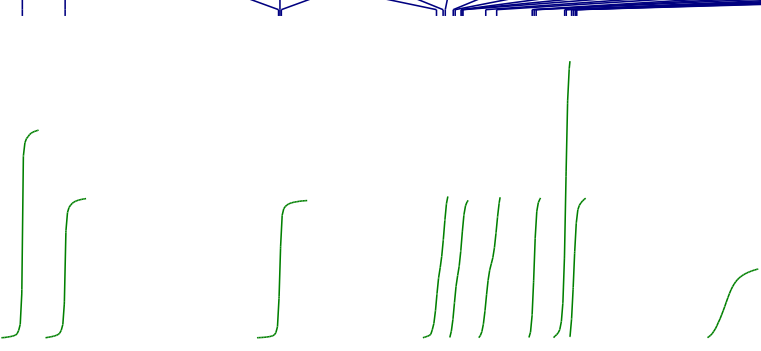

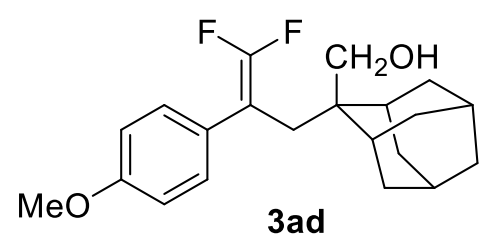

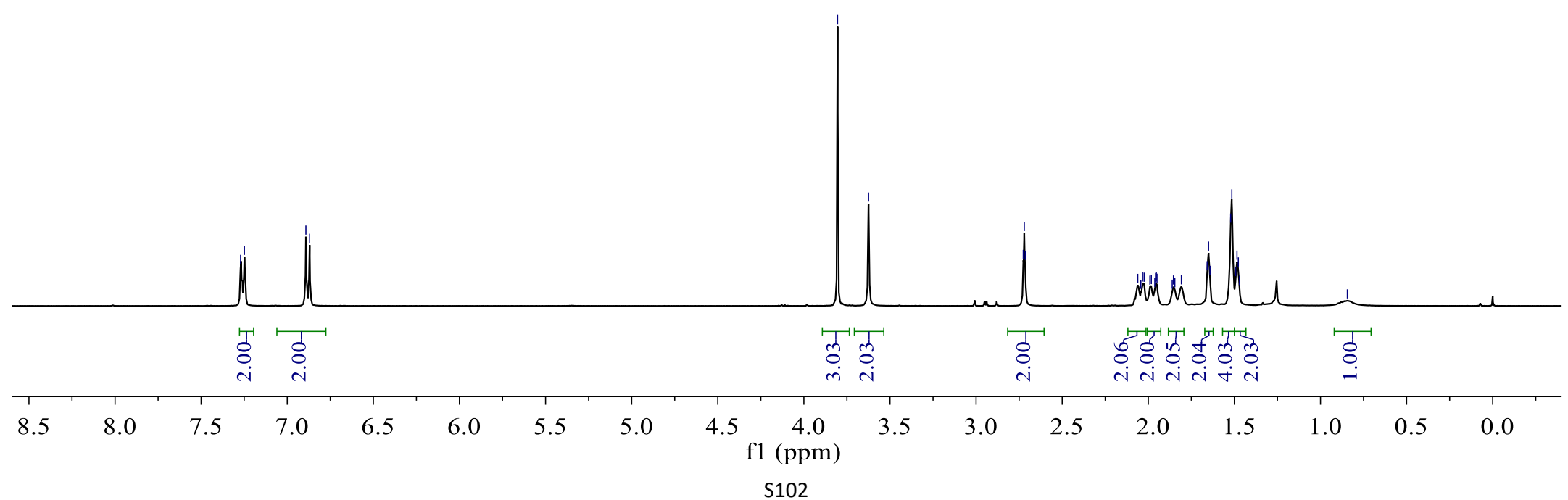


${ }^{13} \mathrm{C}$ NMR-spectrum $\left(101 \mathrm{MHz}, \mathrm{CDCl}_{3}\right)$ of $\mathbf{3 a d}$

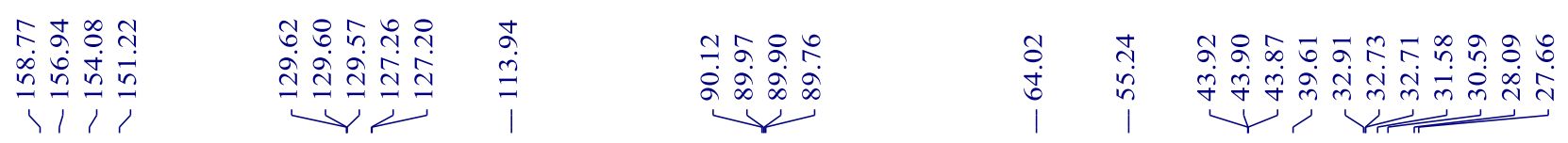
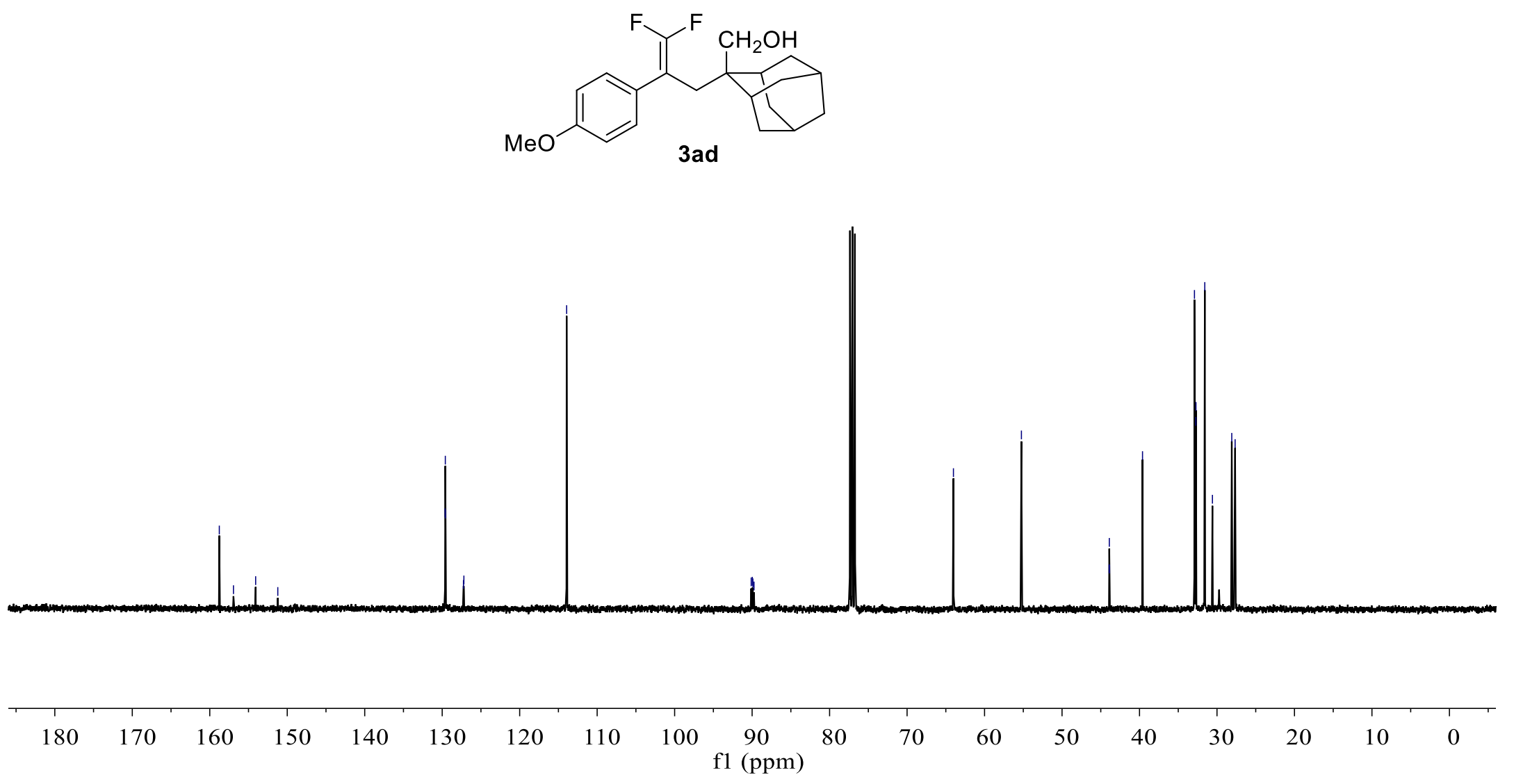

S103 
${ }^{19} \mathrm{~F}$ NMR-spectrum (376 MHz, $\mathrm{CDCl}_{3}$ ) of $\mathbf{3 a d}$
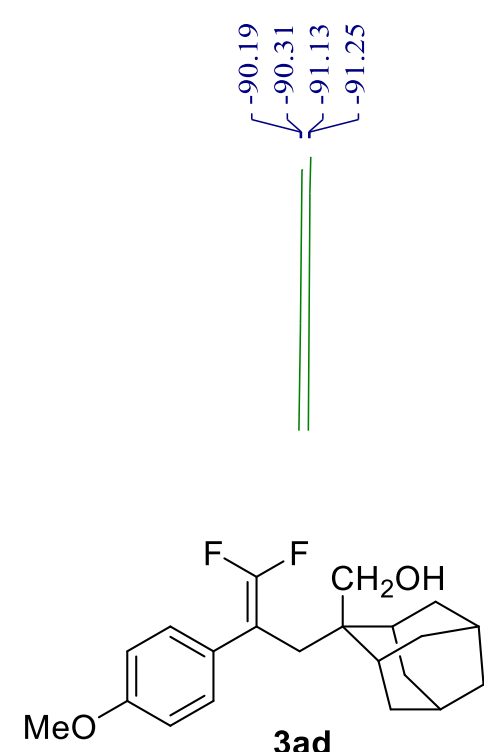

3ad

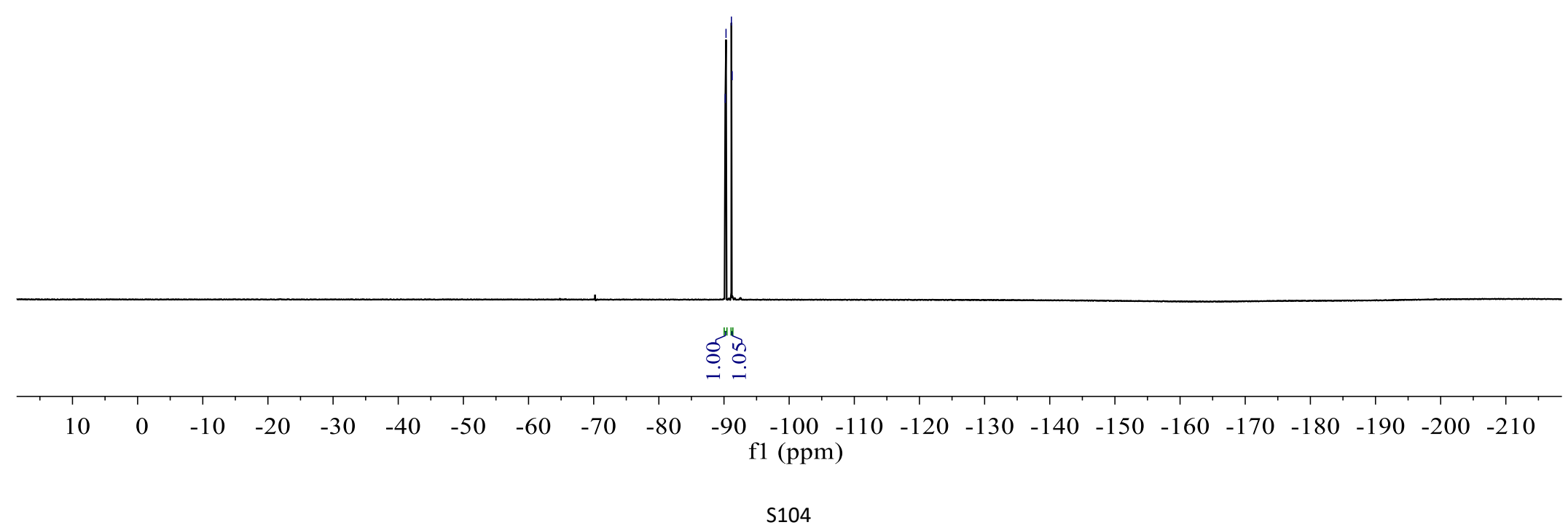


${ }^{1} \mathrm{H}$ NMR-spectrum (400 MHz, $\mathrm{CDCl}_{3}$ ) of 3ae
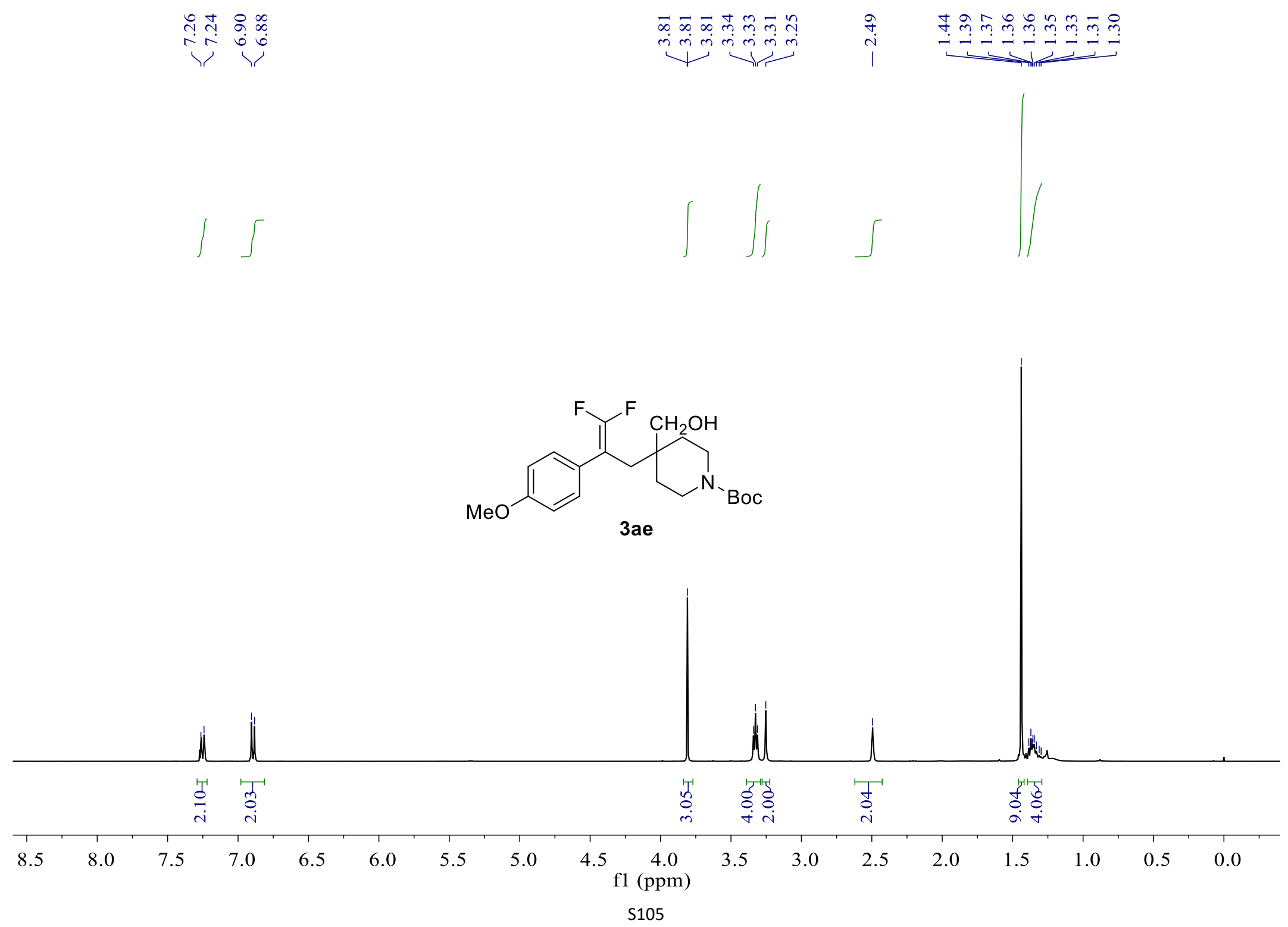
${ }^{13} \mathrm{C}$ NMR-spectrum $\left(101 \mathrm{MHz}, \mathrm{CDCl}_{3}\right)$ of $\mathbf{3 a e}$
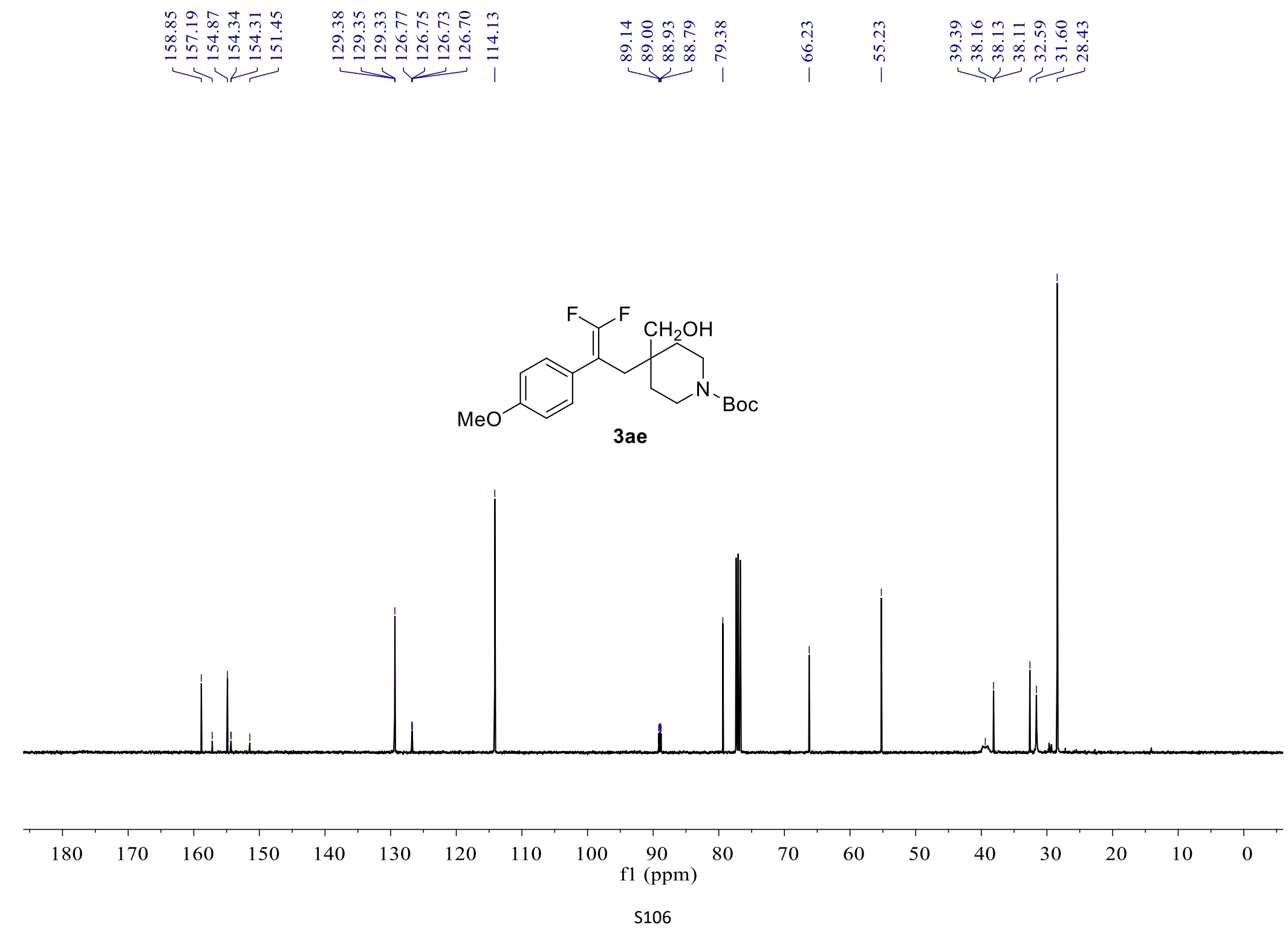
${ }^{19} \mathrm{~F}$ NMR-spectrum $\left(376 \mathrm{MHz}, \mathrm{CDCl}_{3}\right.$ ) of 3ae
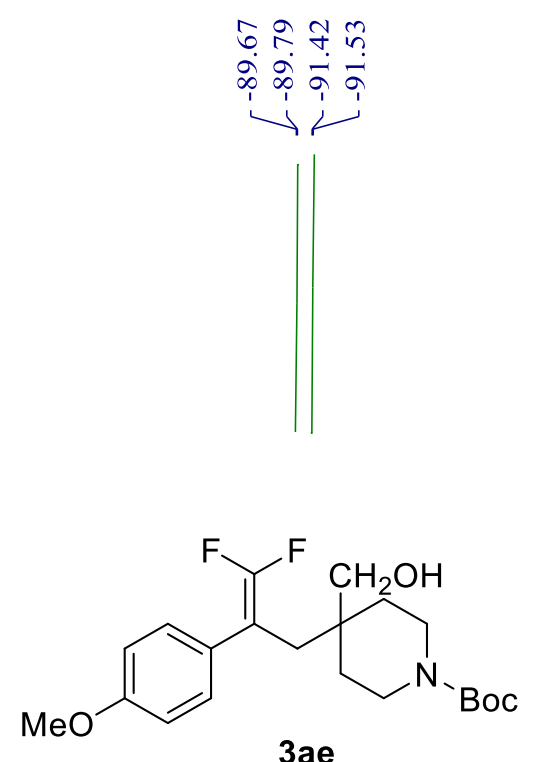

$3 a e$

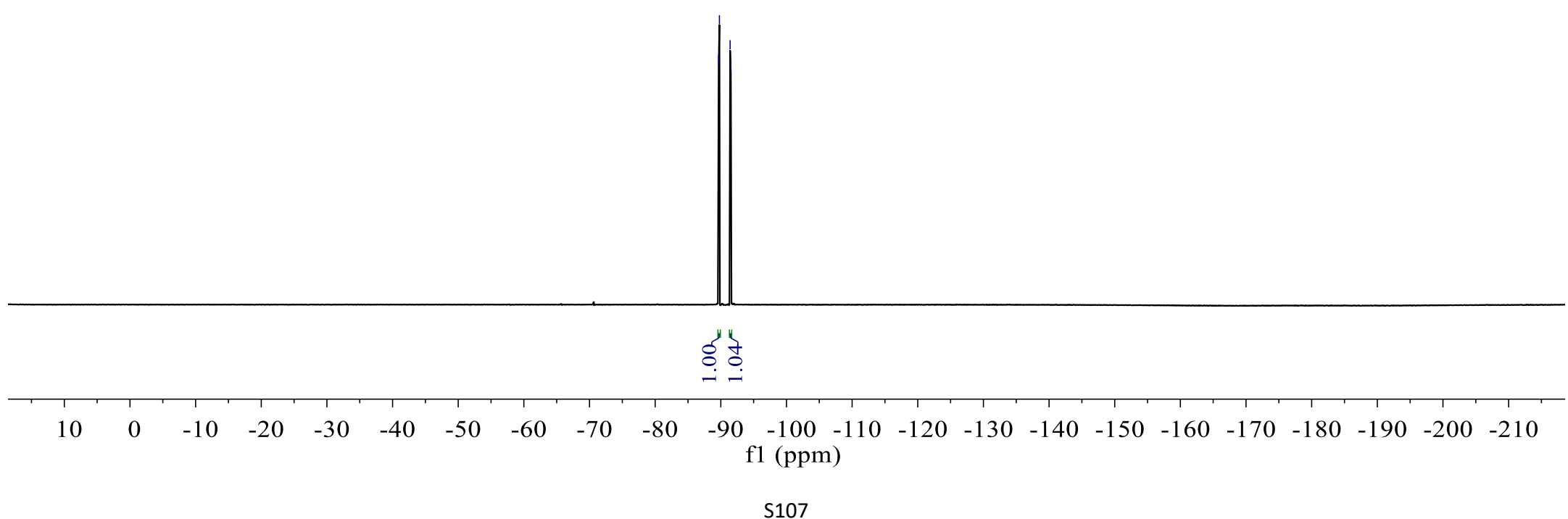




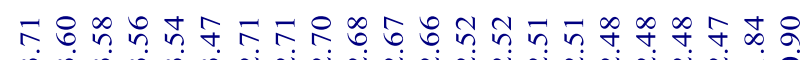

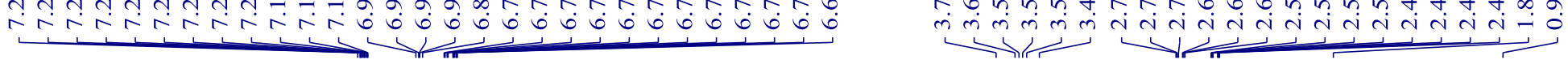
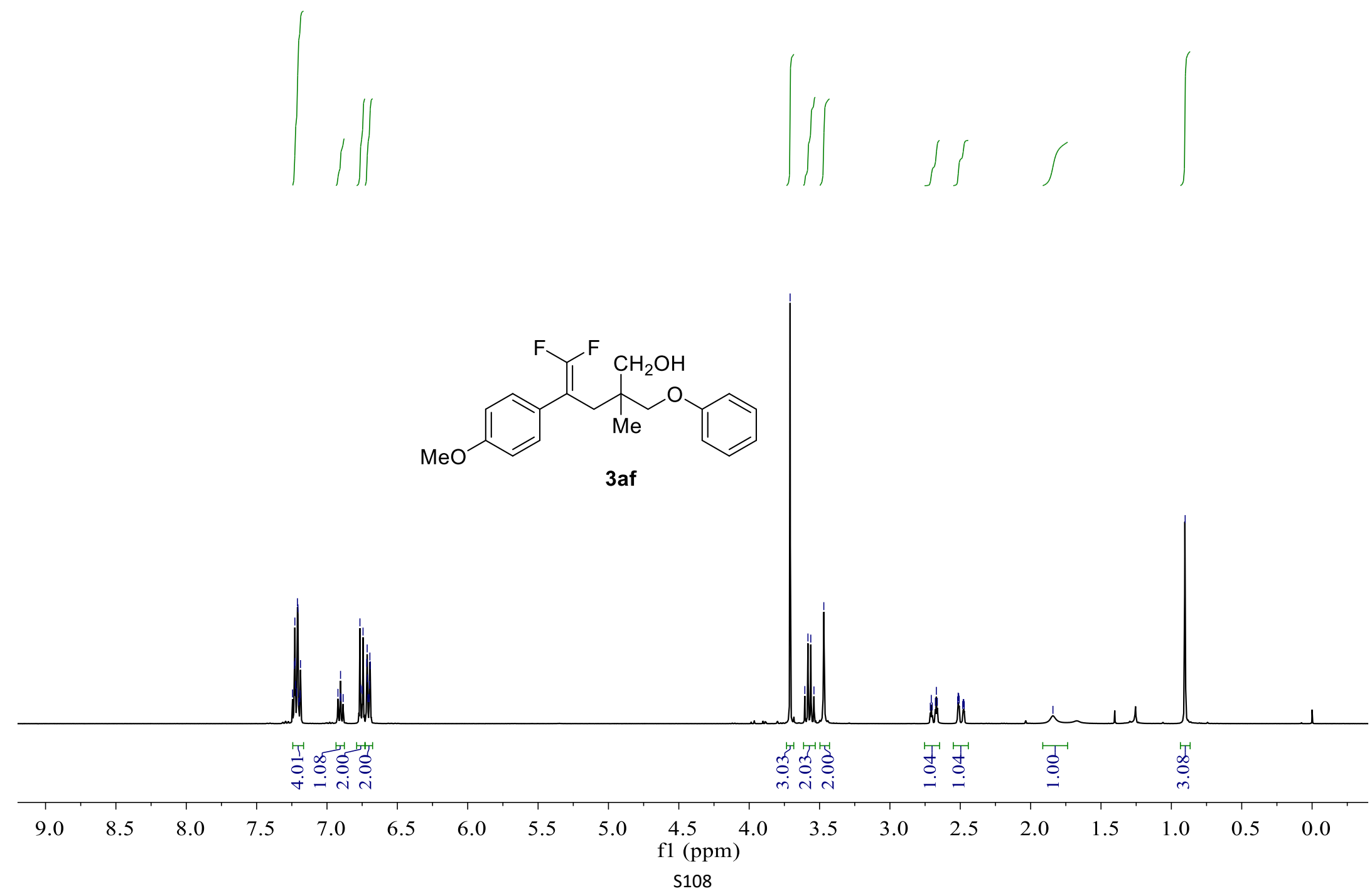
${ }^{13} \mathrm{C}$ NMR-spectrum $\left(101 \mathrm{MHz}, \mathrm{CDCl}_{3}\right)$ of $3 \mathbf{a f}$

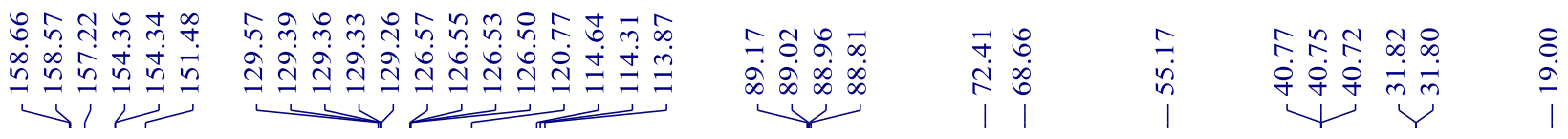

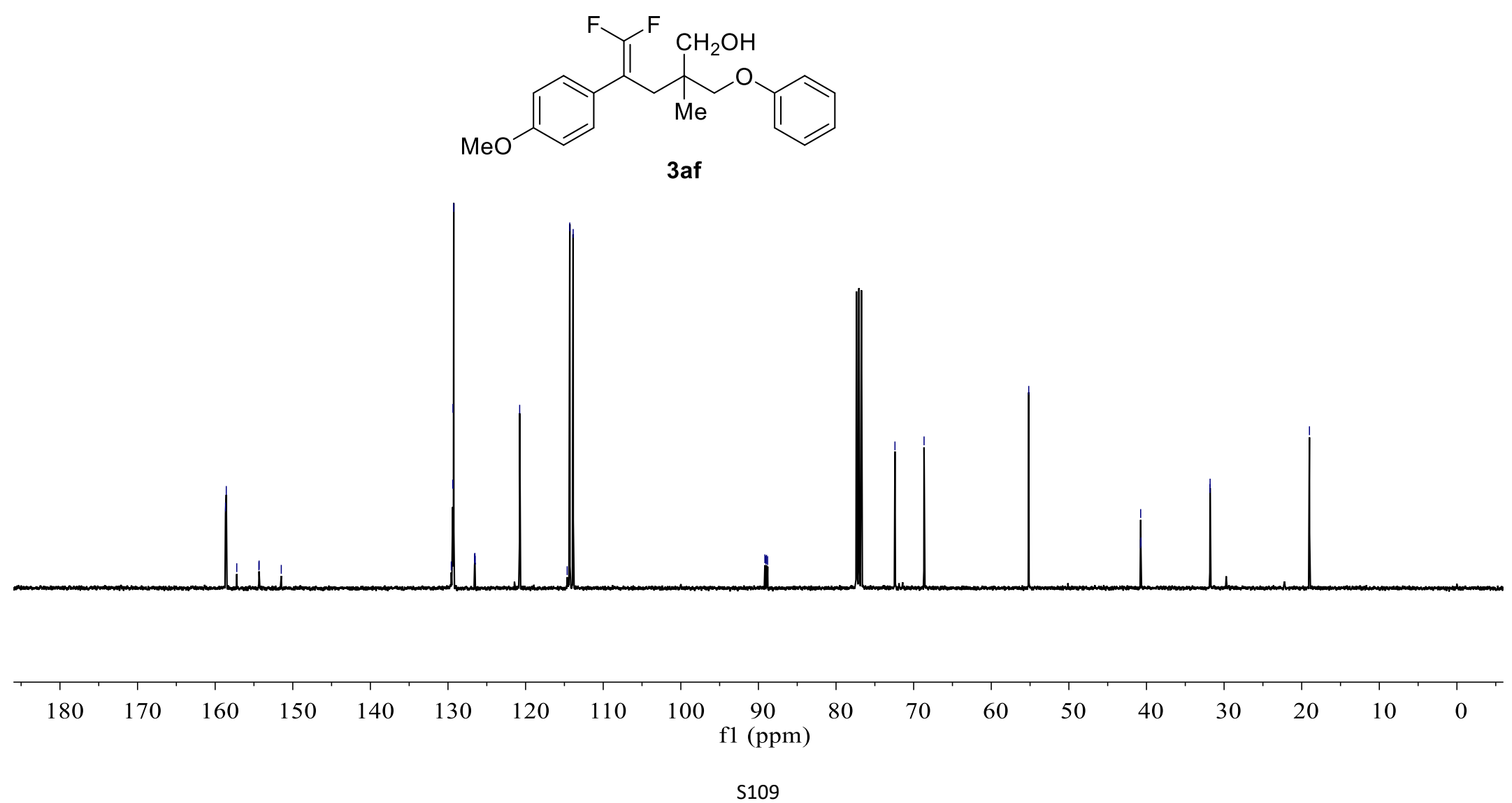


${ }^{19} \mathrm{~F}$ NMR-spectrum $\left(376 \mathrm{MHz}, \mathrm{CDCl}_{3}\right)$ of $\mathbf{3 a f}$
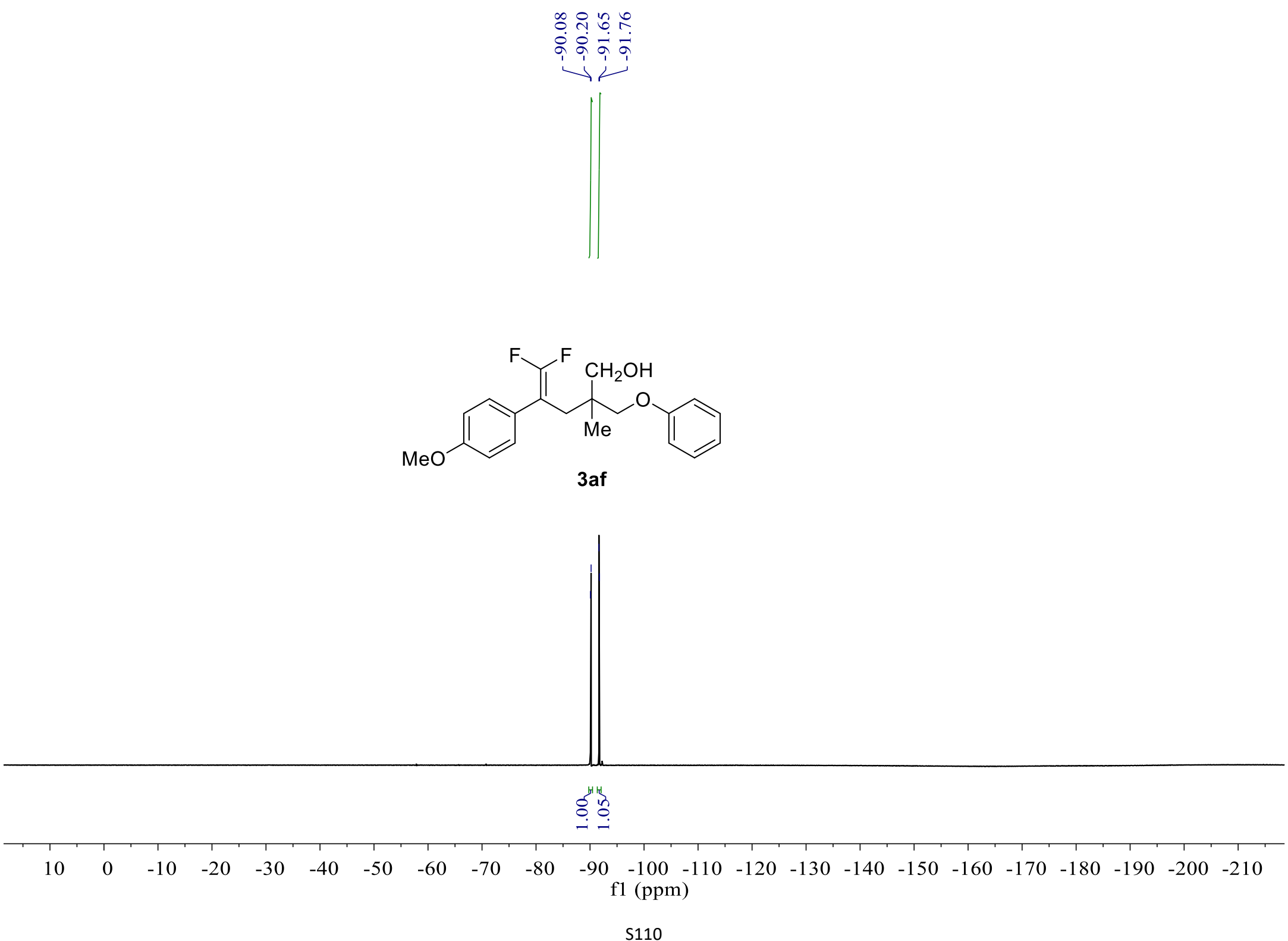
${ }^{1} \mathrm{H}$ NMR-spectrum $\left(400 \mathrm{MHz}, \mathrm{CDCl}_{3}\right)$ of $\mathbf{3 a g}$

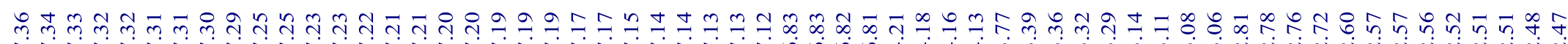

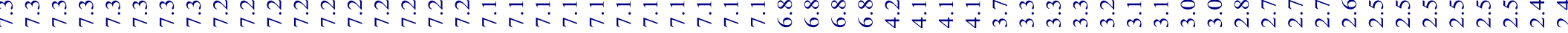
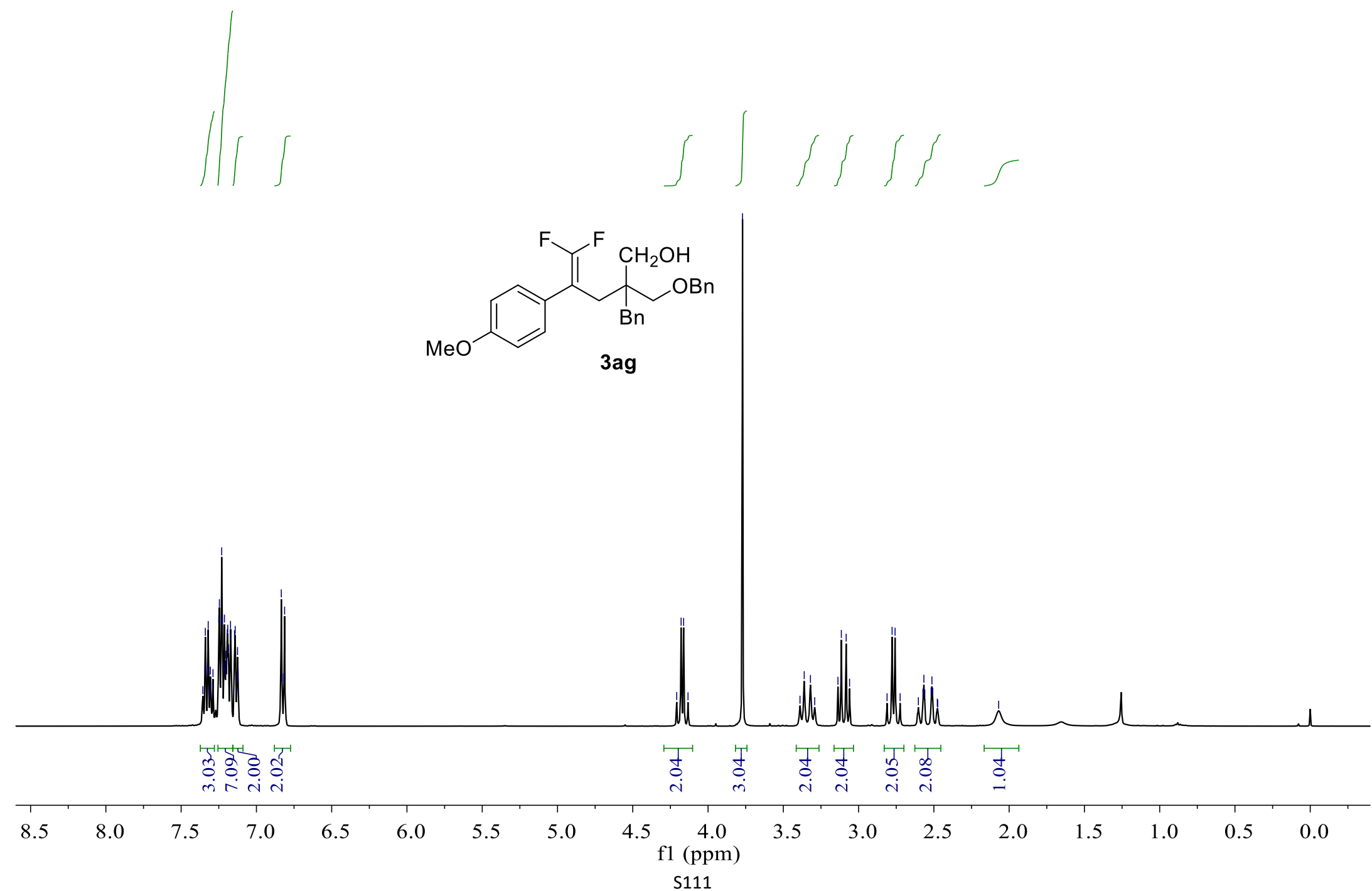
${ }^{13} \mathrm{C}$ NMR-spectrum (101 MHz, $\left.\mathrm{CDCl}_{3}\right)$ of $\mathbf{3 a g}$
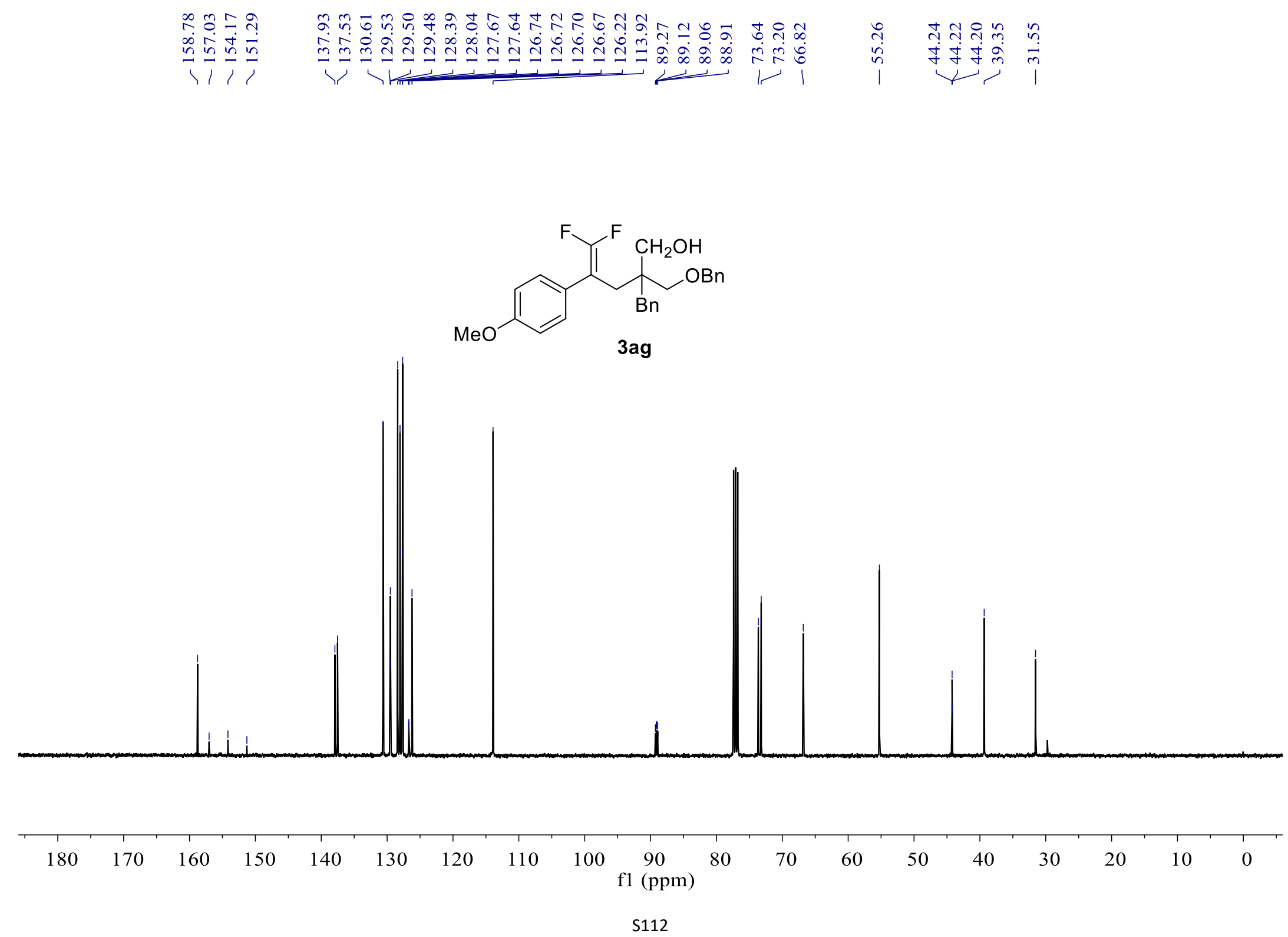
${ }^{19} \mathrm{~F}$ NMR-spectrum (376 MHz, $\mathrm{CDCl}_{3}$ ) of $\mathbf{3 a g}$
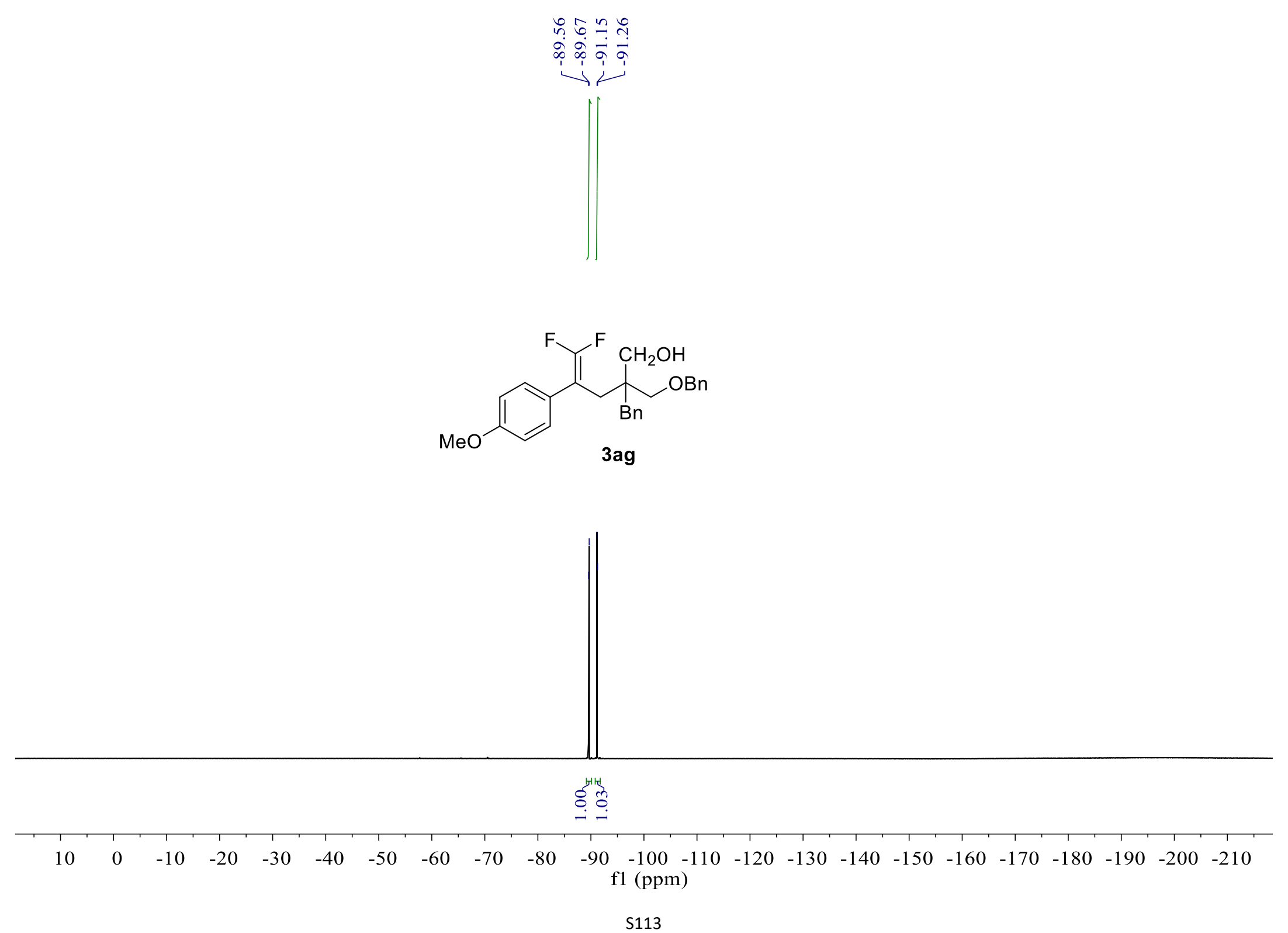


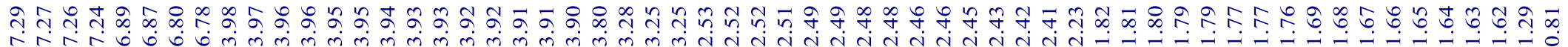

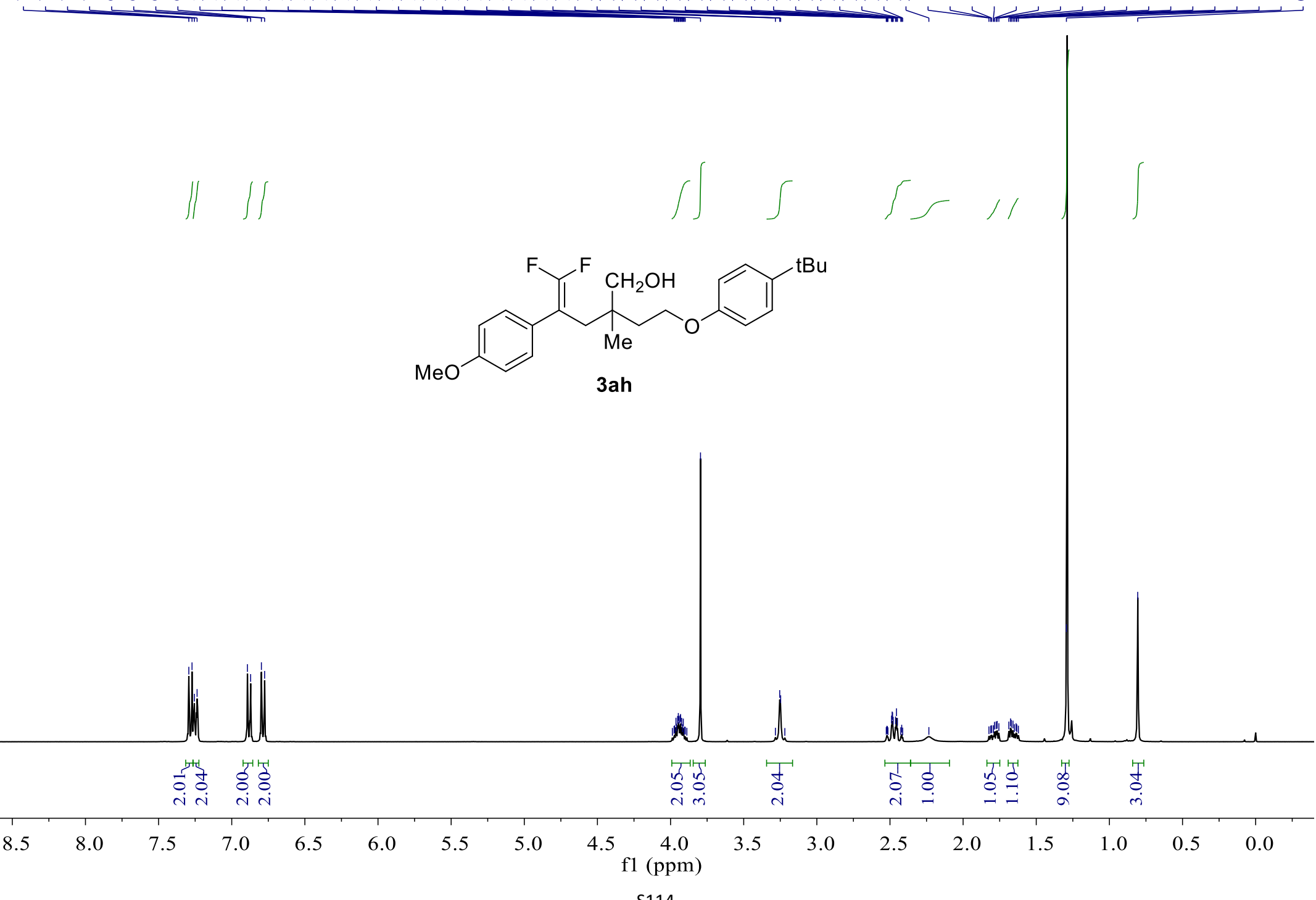


${ }^{13} \mathrm{C}$ NMR-spectrum $\left(101 \mathrm{MHz}, \mathrm{CDCl}_{3}\right)$ of $\mathbf{3 a h}$
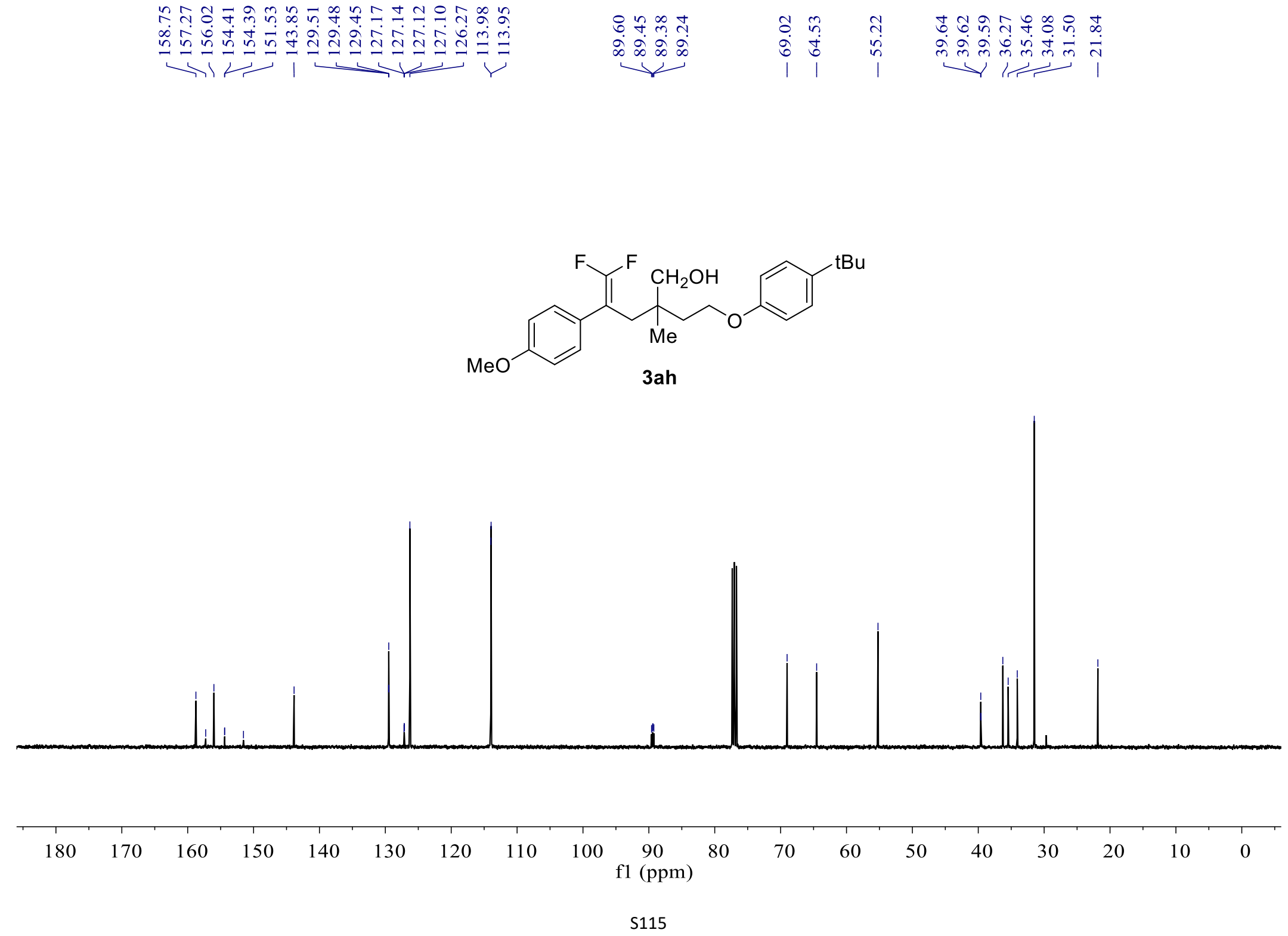
${ }^{19} \mathrm{~F}$ NMR-spectrum $\left(376 \mathrm{MHz}, \mathrm{CDCl}_{3}\right)$ of $\mathbf{3 a h}$

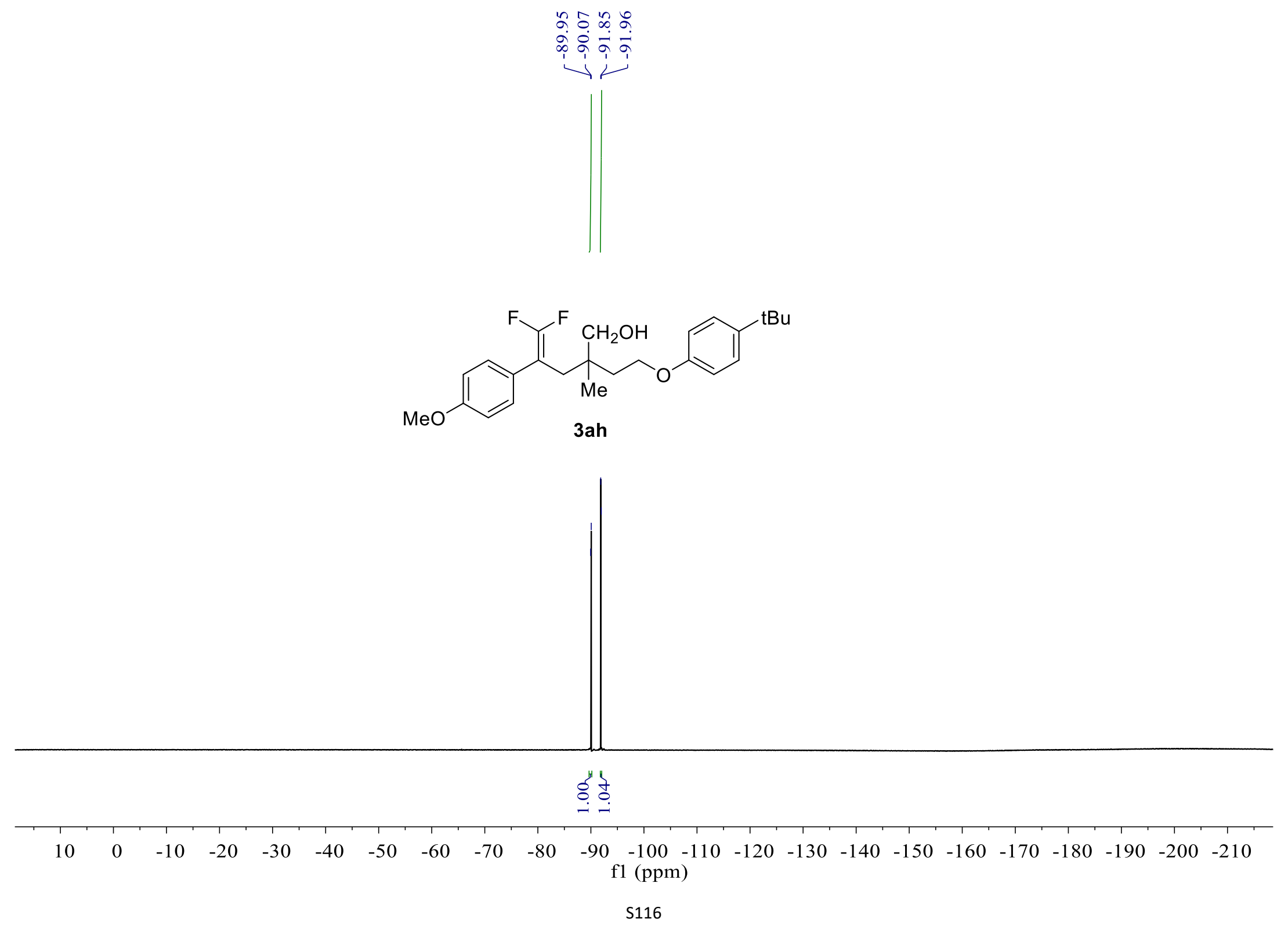




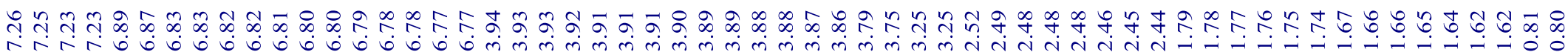

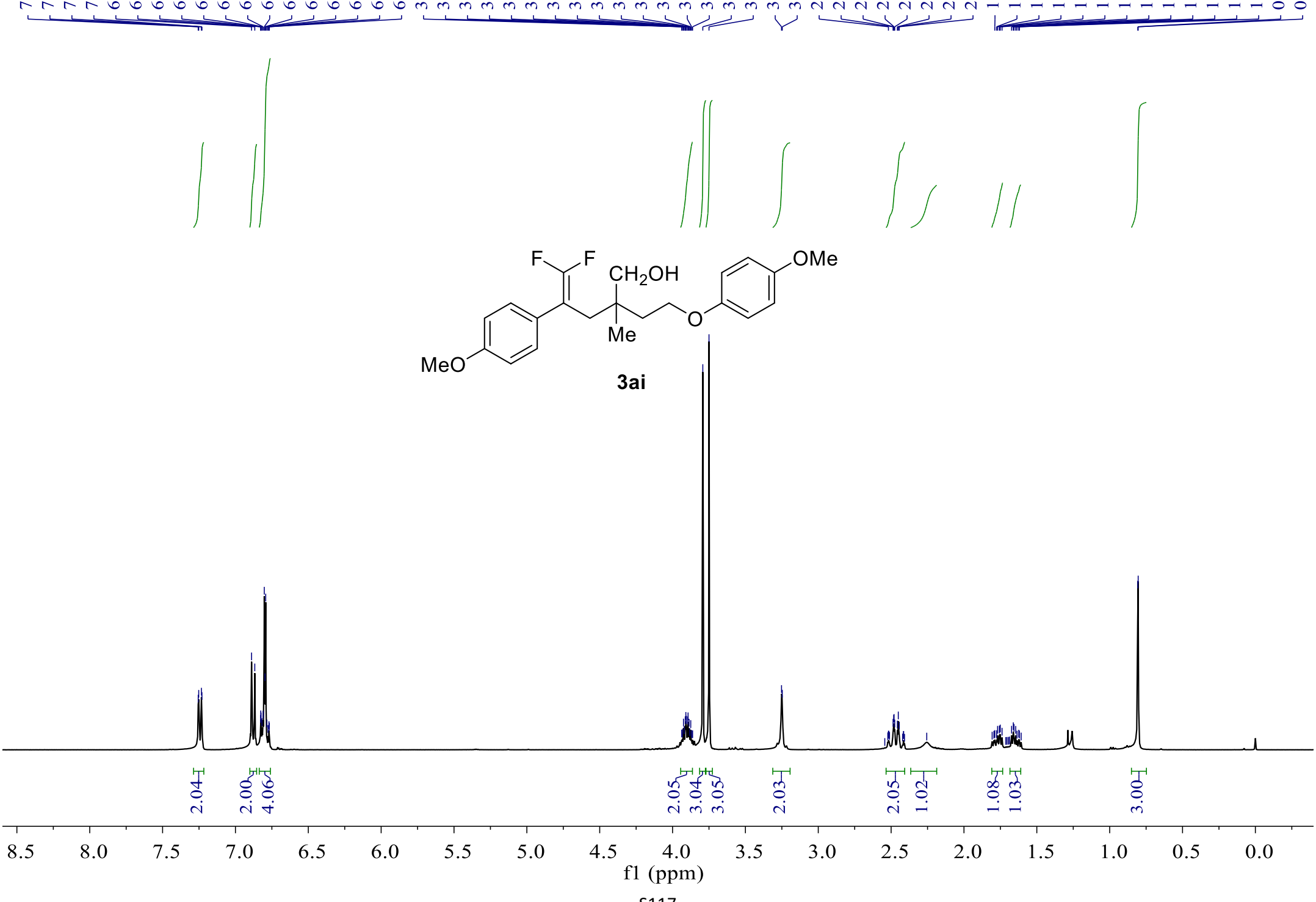


${ }^{13} \mathrm{C}$ NMR-spectrum $\left(101 \mathrm{MHz}, \mathrm{CDCl}_{3}\right)$ of 3ai
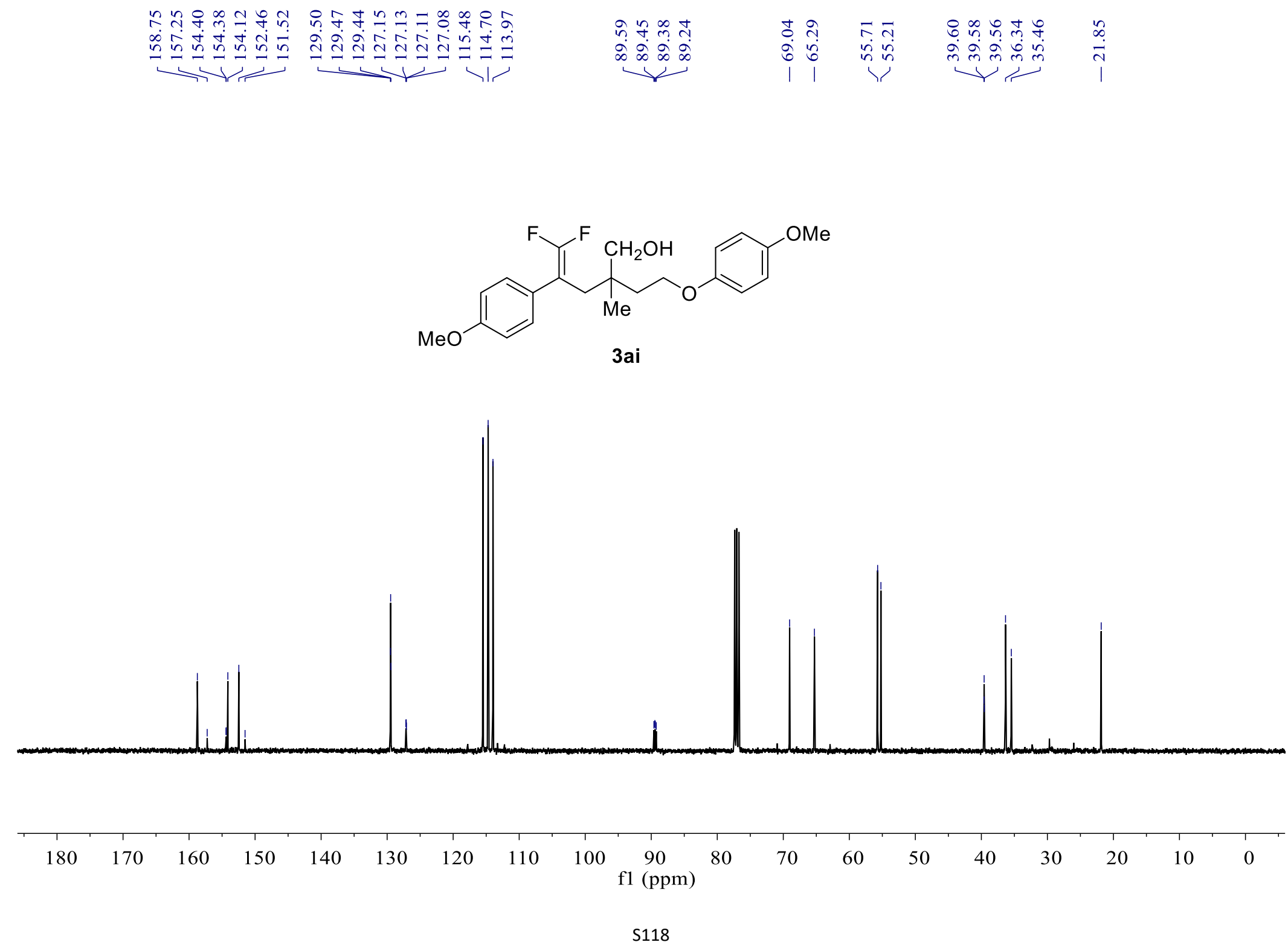
${ }^{19} \mathrm{~F} \mathrm{NMR}$-spectrum $\left(376 \mathrm{MHz}, \mathrm{CDCl}_{3}\right)$ of 3ai
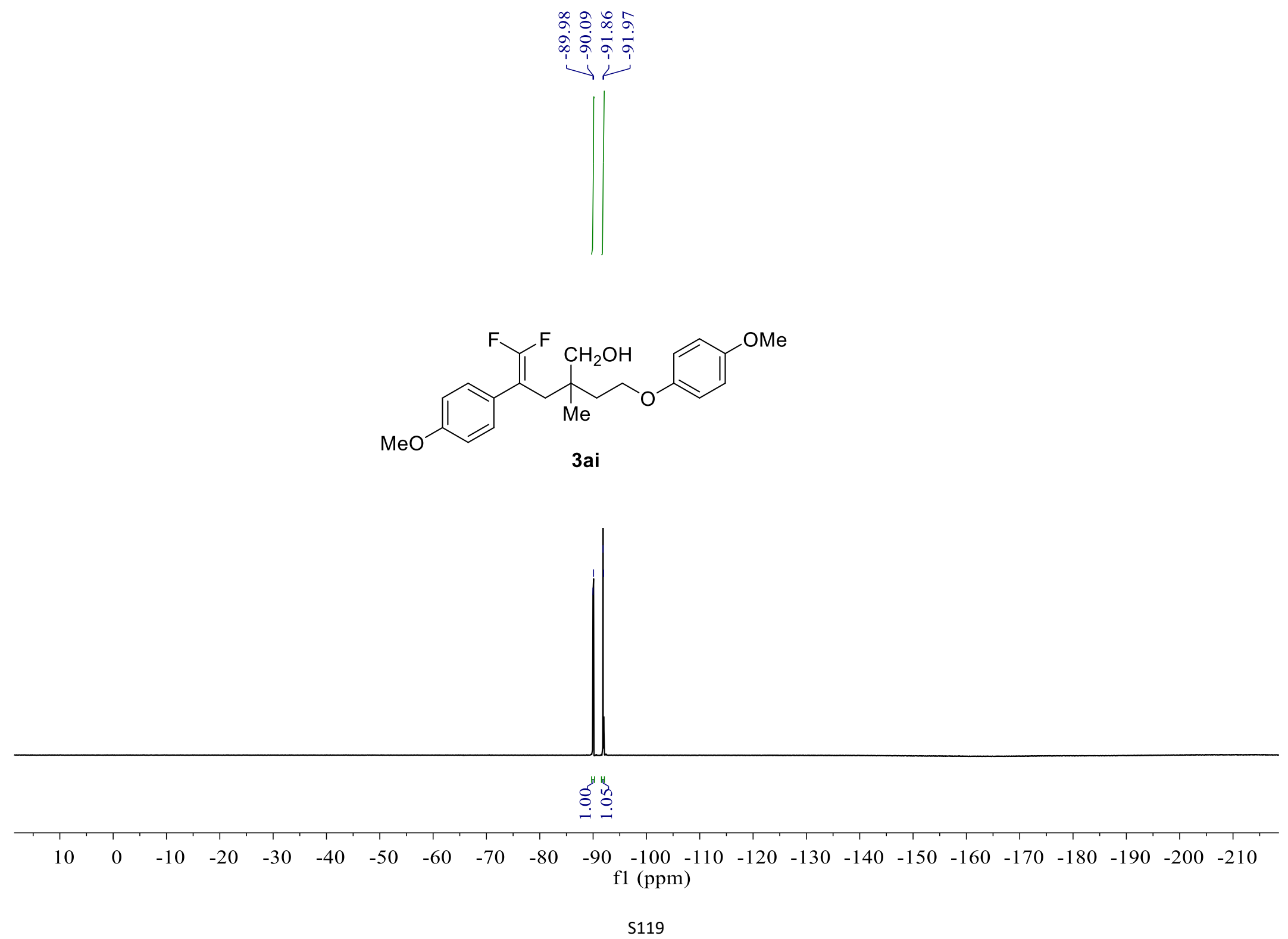
${ }^{1} \mathrm{H}$ NMR-spectrum $\left(400 \mathrm{MHz}, \mathrm{CDCl}_{3}\right)$ of $\mathbf{3 a j}$
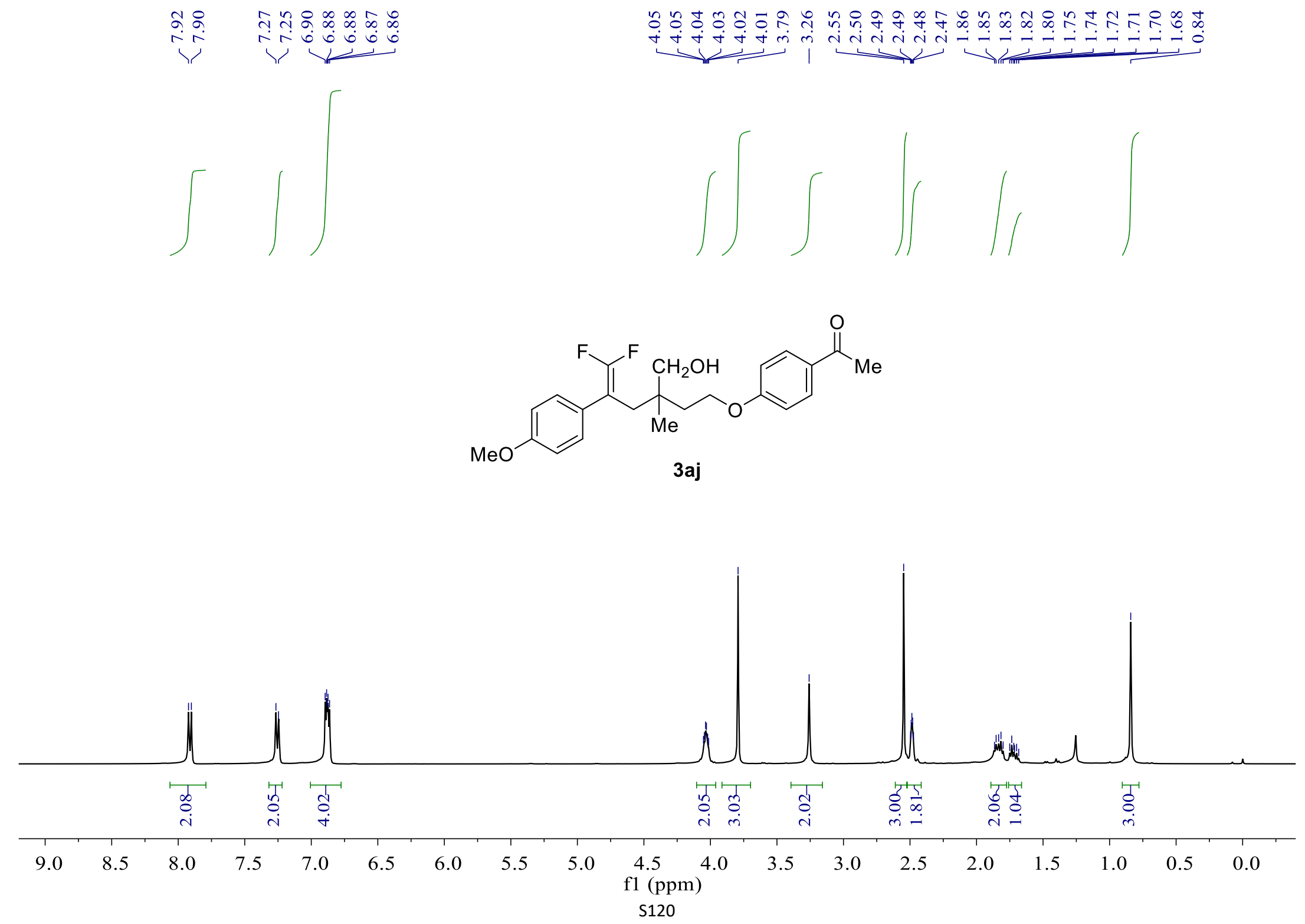
${ }^{13} \mathrm{C}$ NMR-spectrum $\left(101 \mathrm{MHz}, \mathrm{CDCl}_{3}\right)$ of $\mathbf{3 a j}$
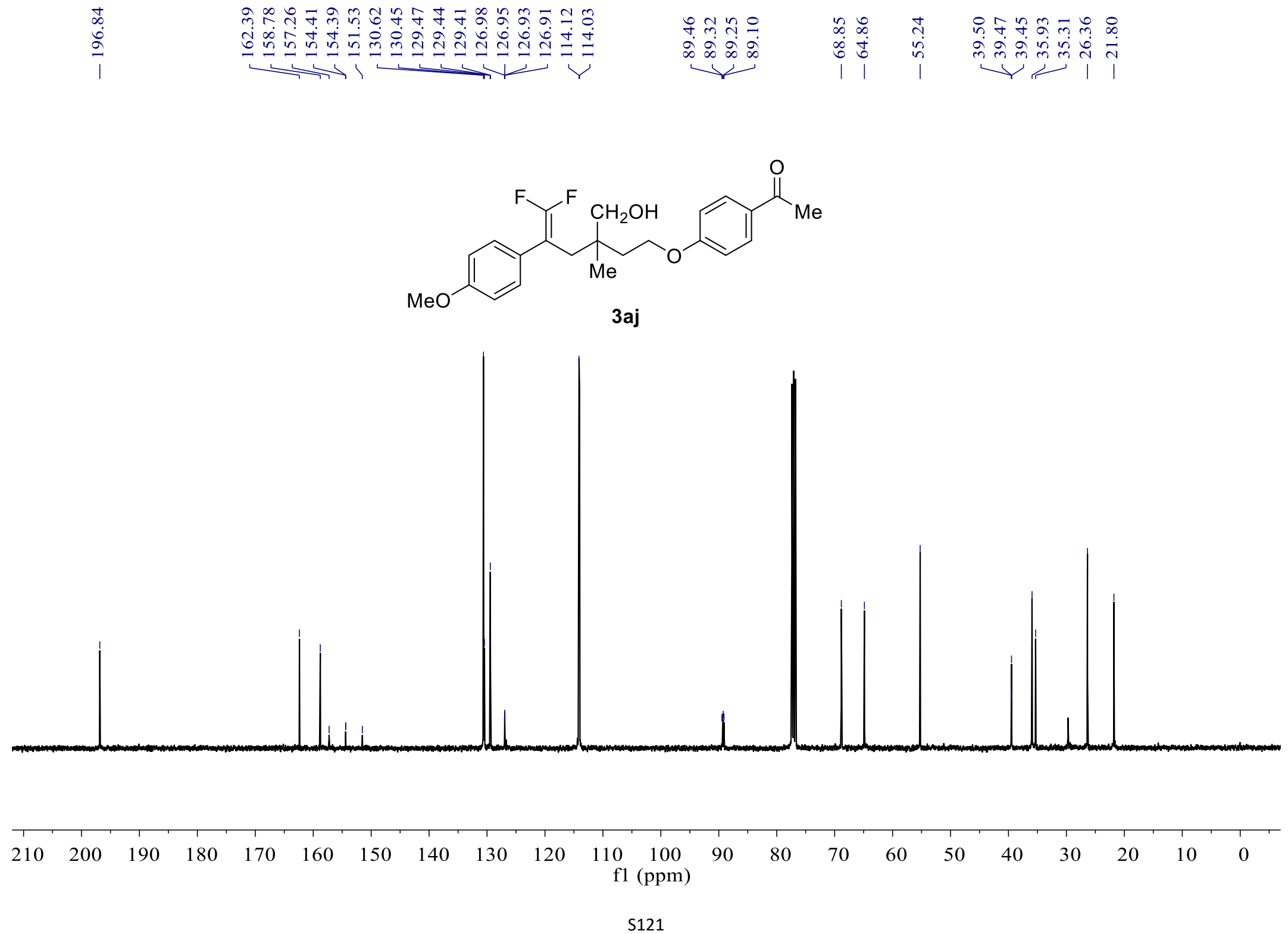
${ }^{19} \mathrm{~F} \mathrm{NMR}$-spectrum $\left(376 \mathrm{MHz}, \mathrm{CDCl}_{3}\right)$ of $\mathbf{3 a j}$

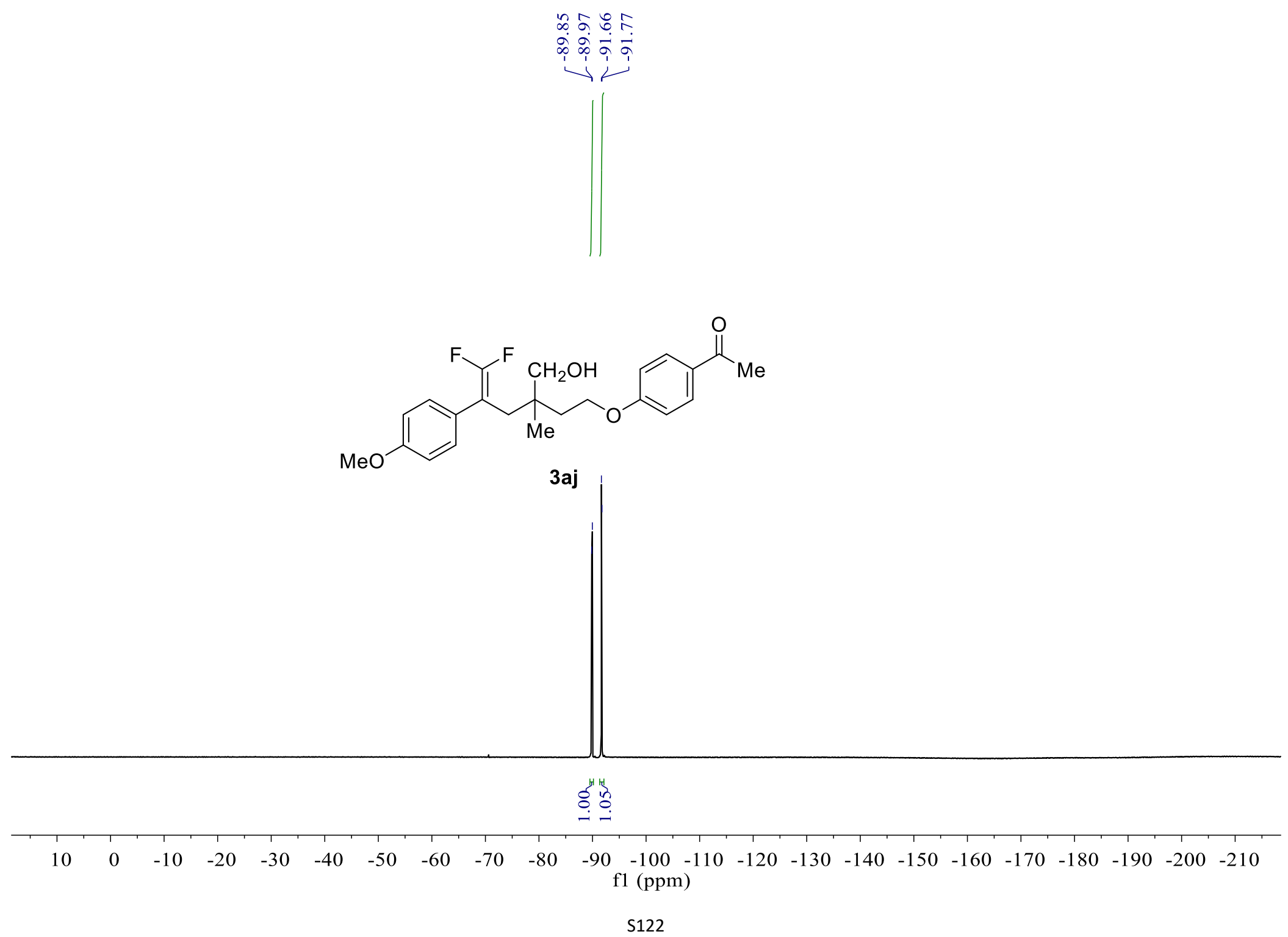


${ }^{1} \mathrm{H}$ NMR-spectrum $\left(400 \mathrm{MHz}, \mathrm{CDCl}_{3}\right)$ of $\mathbf{3 a k}$

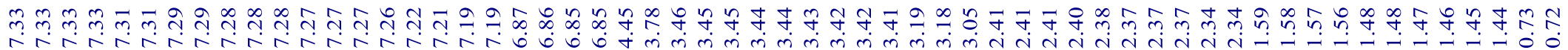
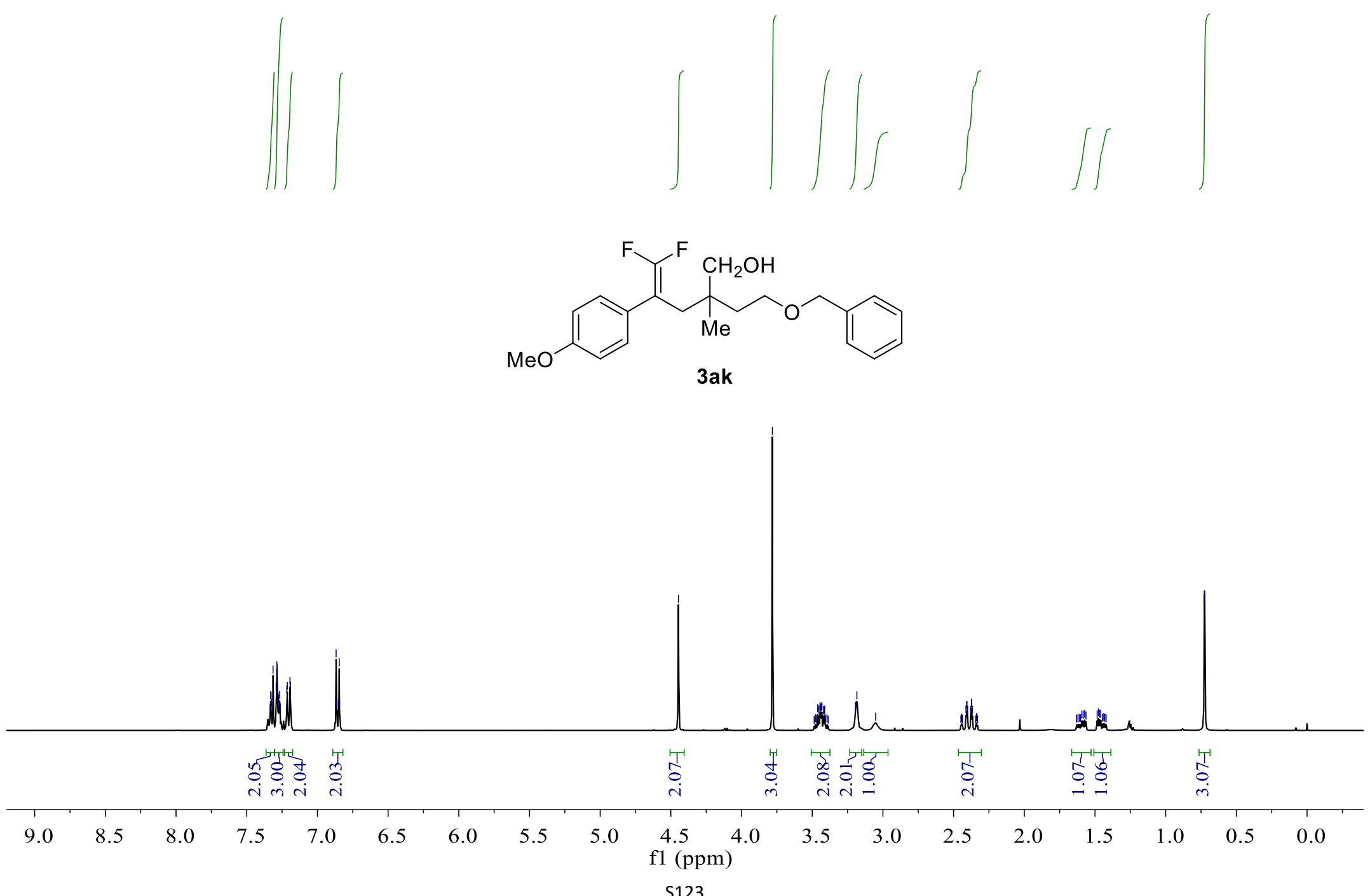

S123 
${ }^{13} \mathrm{C}$ NMR-spectrum (101 MHz, $\left.\mathrm{CDCl}_{3}\right)$ of 3ak
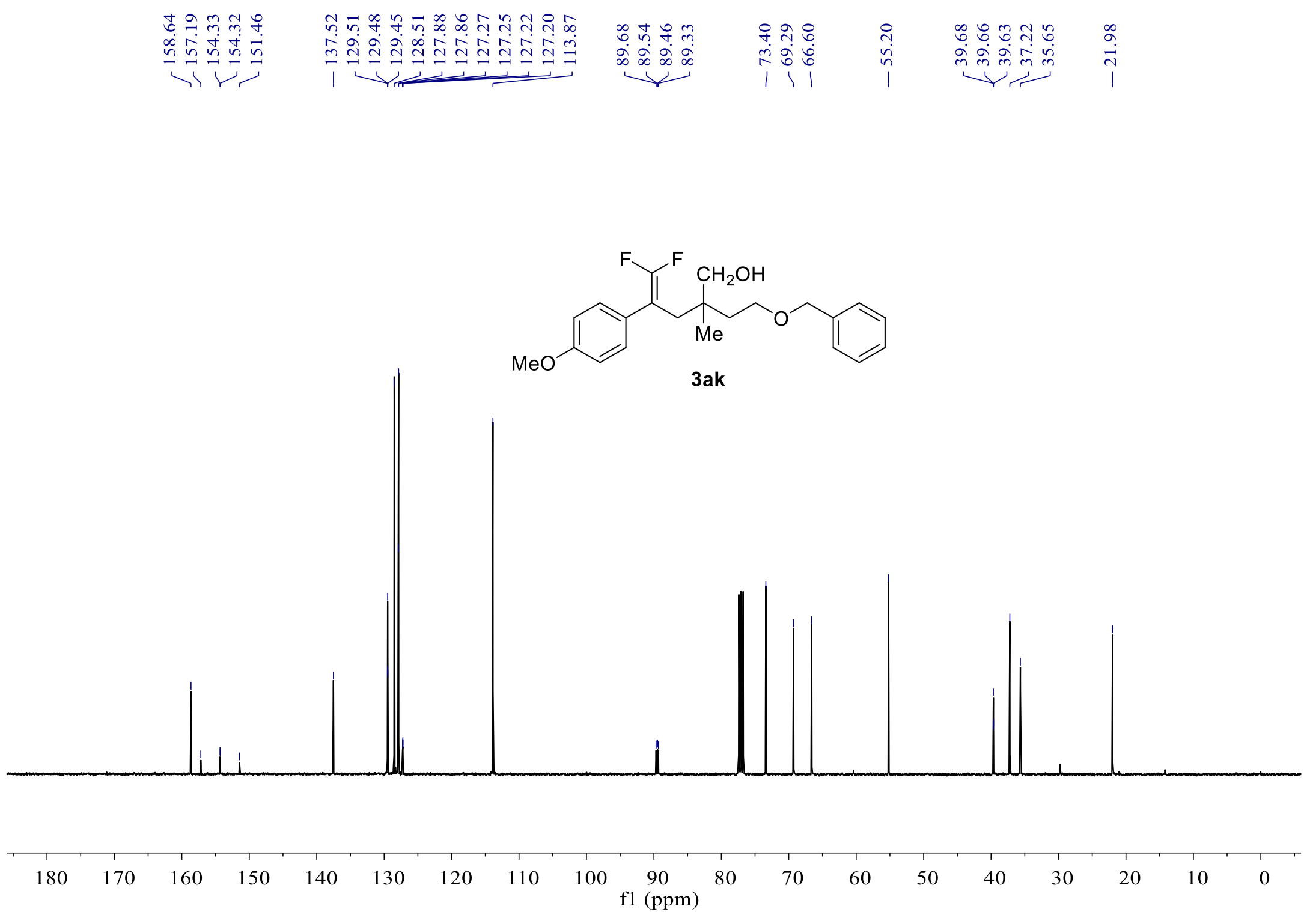
${ }^{19} \mathrm{~F}$ NMR-spectrum (376 MHz, $\mathrm{CDCl}_{3}$ ) of 3ak
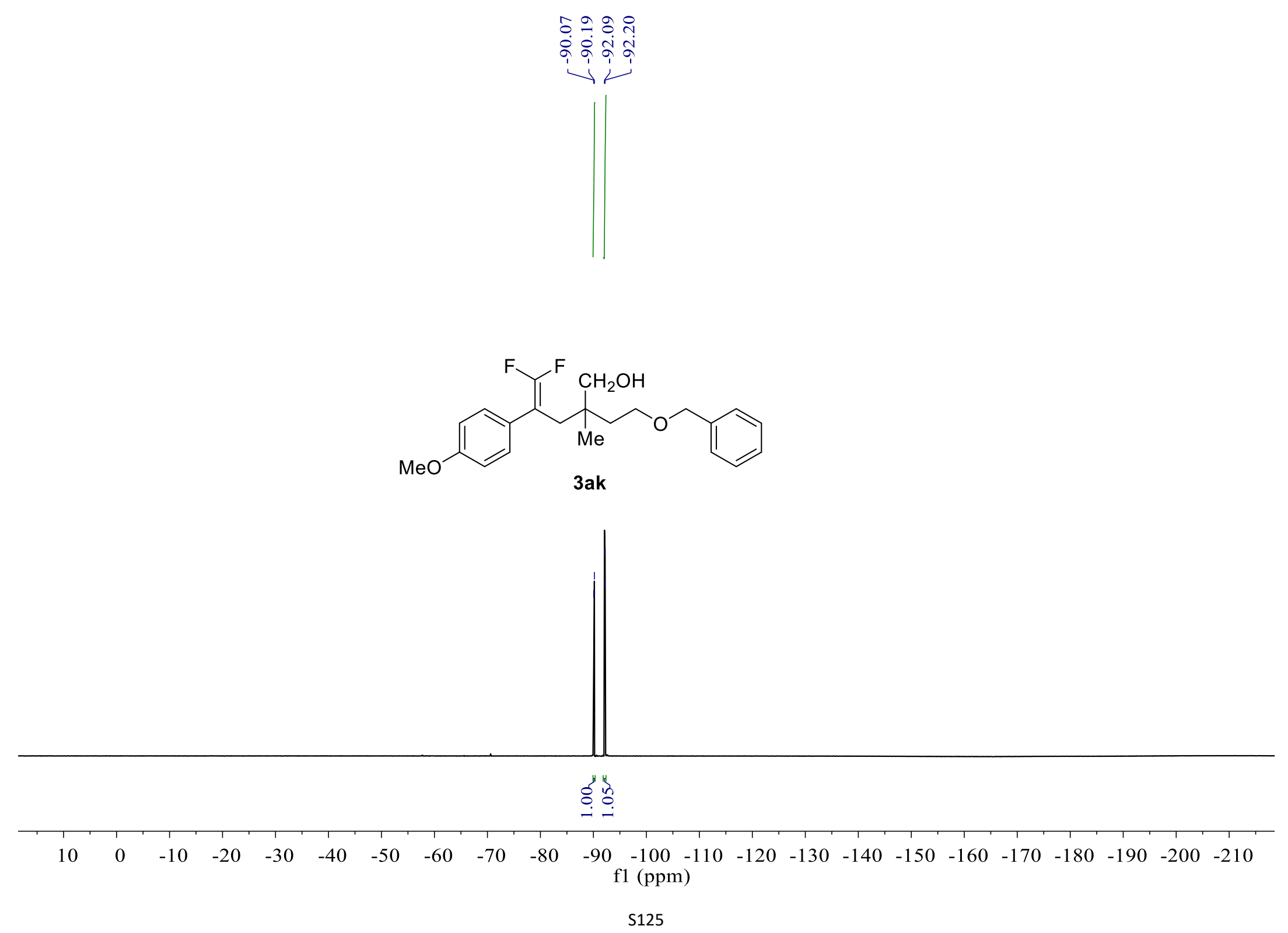
${ }^{1} \mathrm{H}$ NMR-spectrum $\left(400 \mathrm{MHz}, \mathrm{CDCl}_{3}\right)$ of $\mathbf{3 a l}$

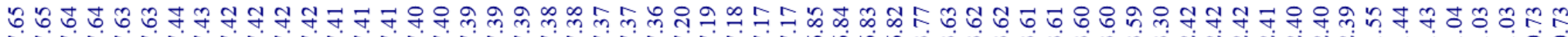

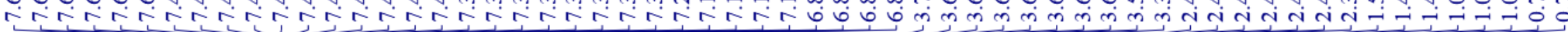

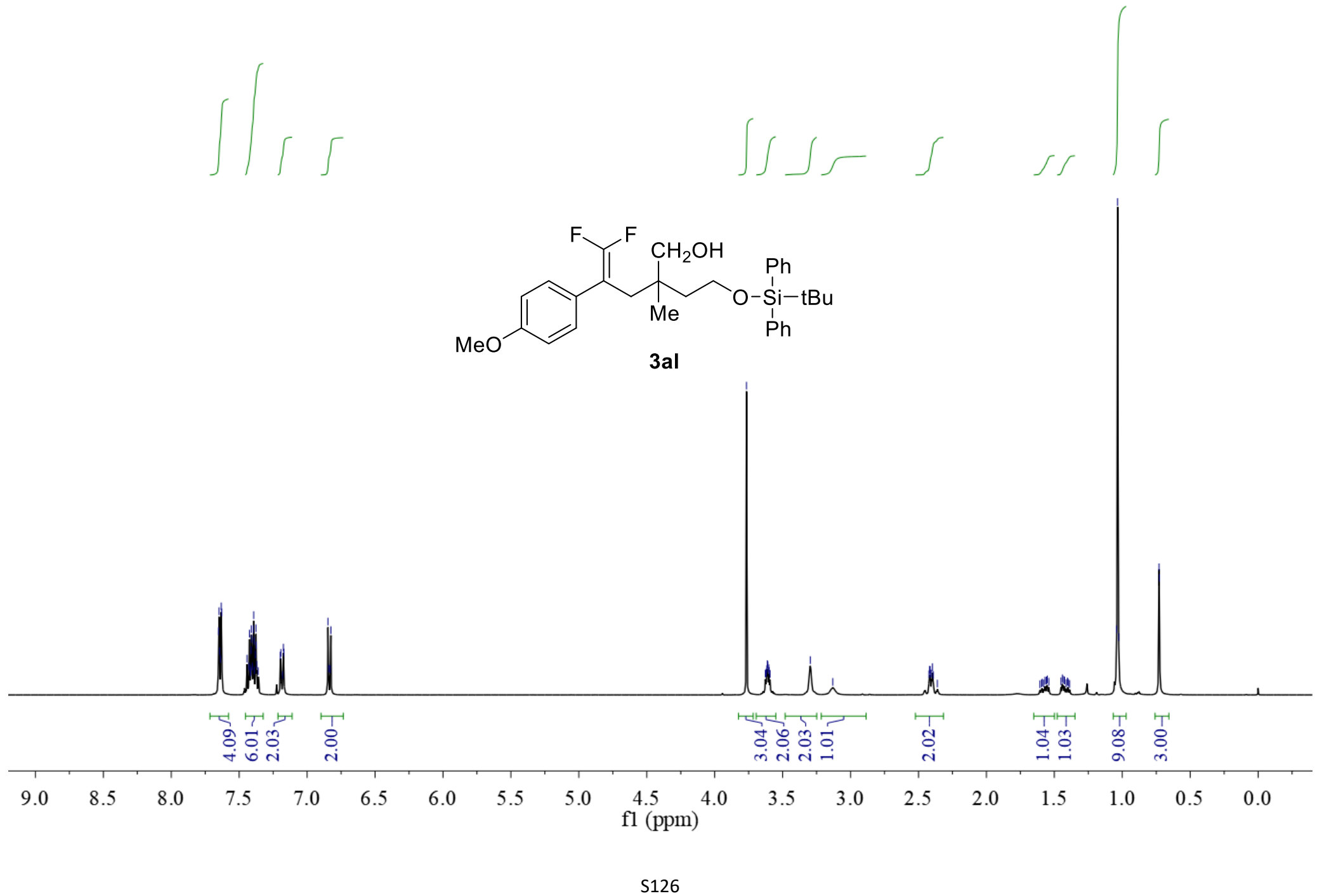



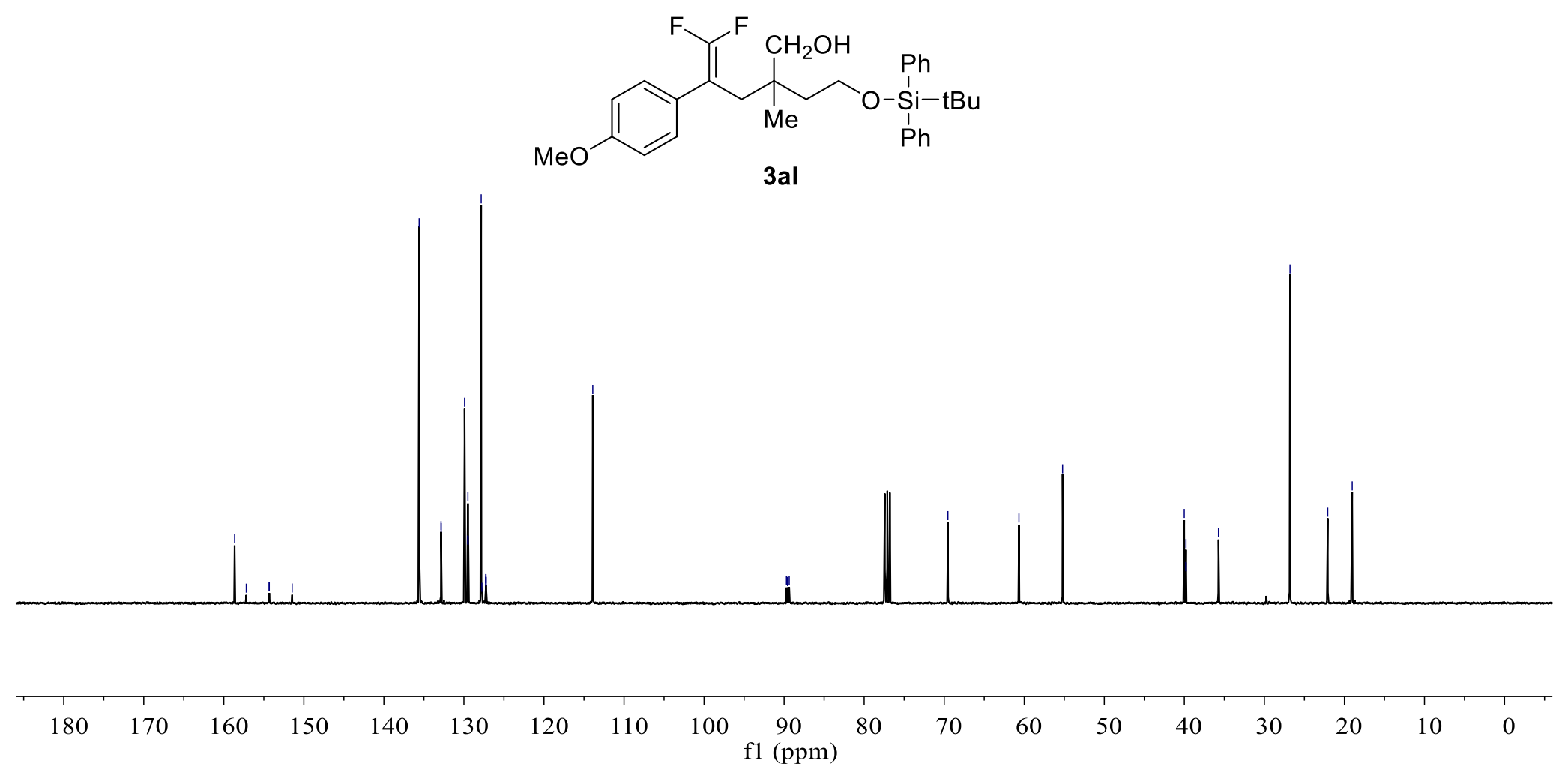
${ }^{19} \mathrm{~F} \mathrm{NMR}$-spectrum $\left(376 \mathrm{MHz}, \mathrm{CDCl}_{3}\right)$ of $\mathbf{3 a l}$

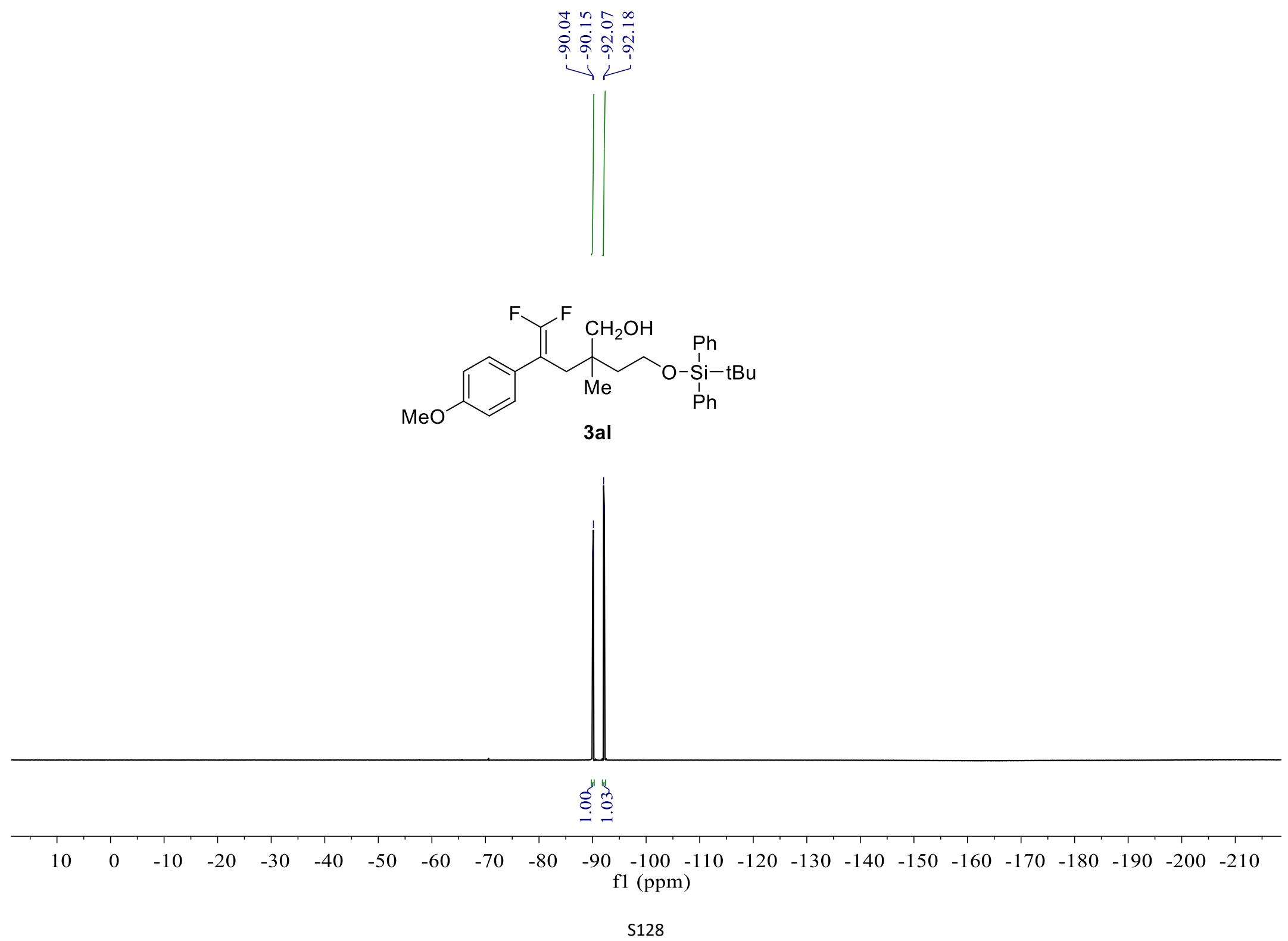


${ }^{1} \mathrm{H}$ NMR-spectrum $\left(400 \mathrm{MHz}, \mathrm{CDCl}_{3}\right)$ of $\mathbf{3 a m}$
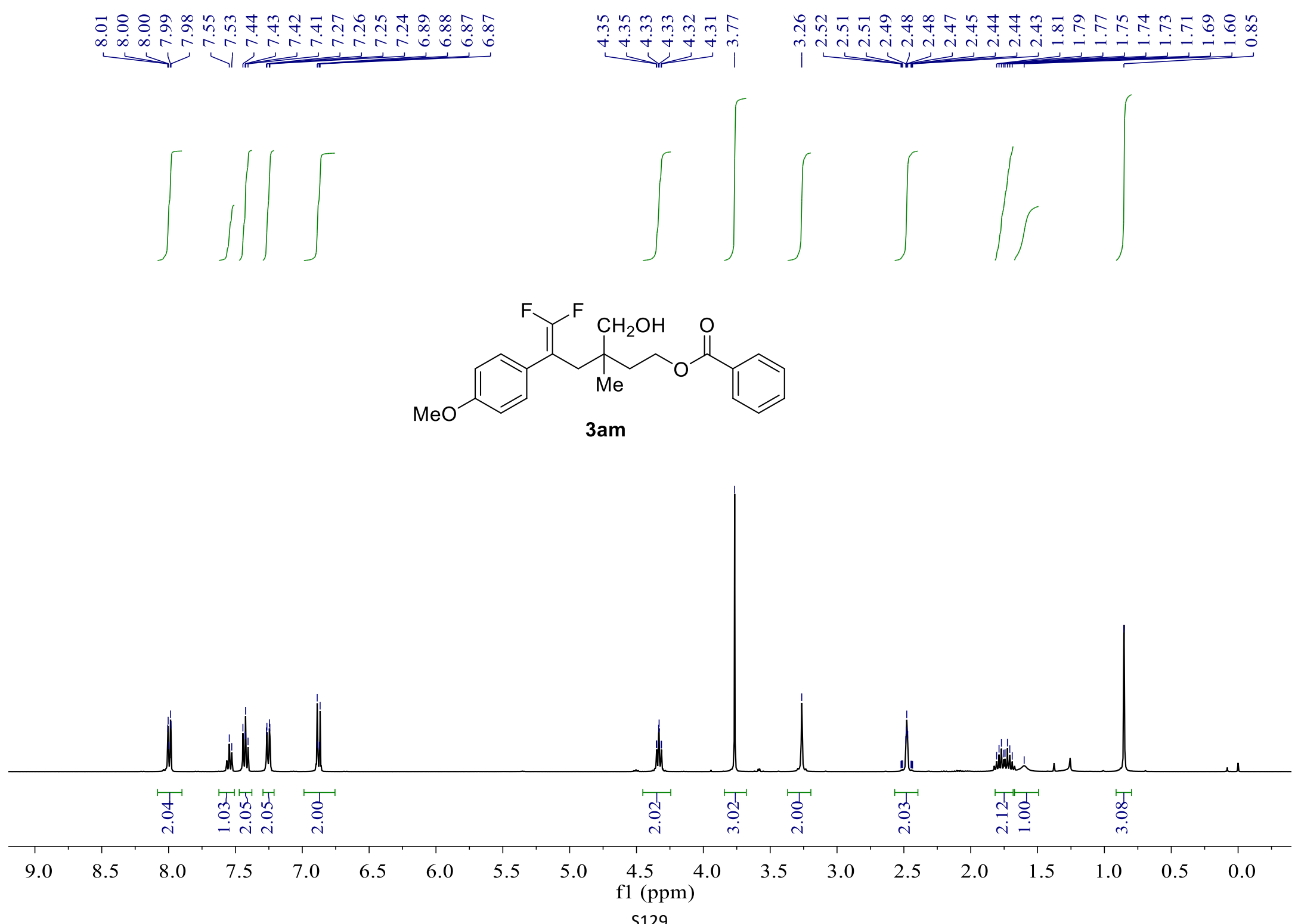
${ }^{13} \mathrm{C}$ NMR-spectrum (101 MHz, $\mathrm{CDCl}_{3}$ ) of 3am
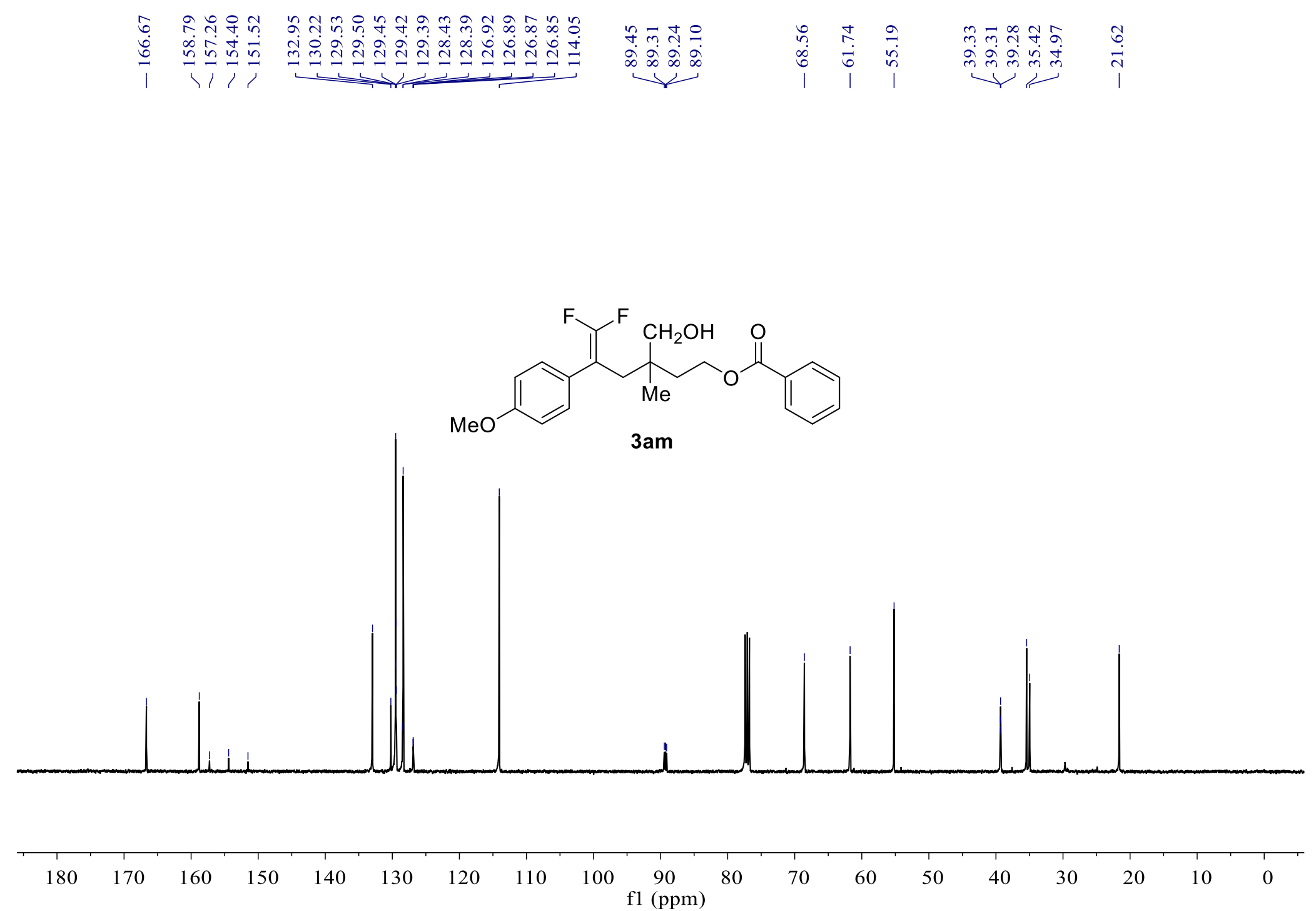
${ }^{19} \mathrm{~F}$ NMR-spectrum $\left(376 \mathrm{MHz}, \mathrm{CDCl}_{3}\right)$ of $\mathbf{3 a m}$
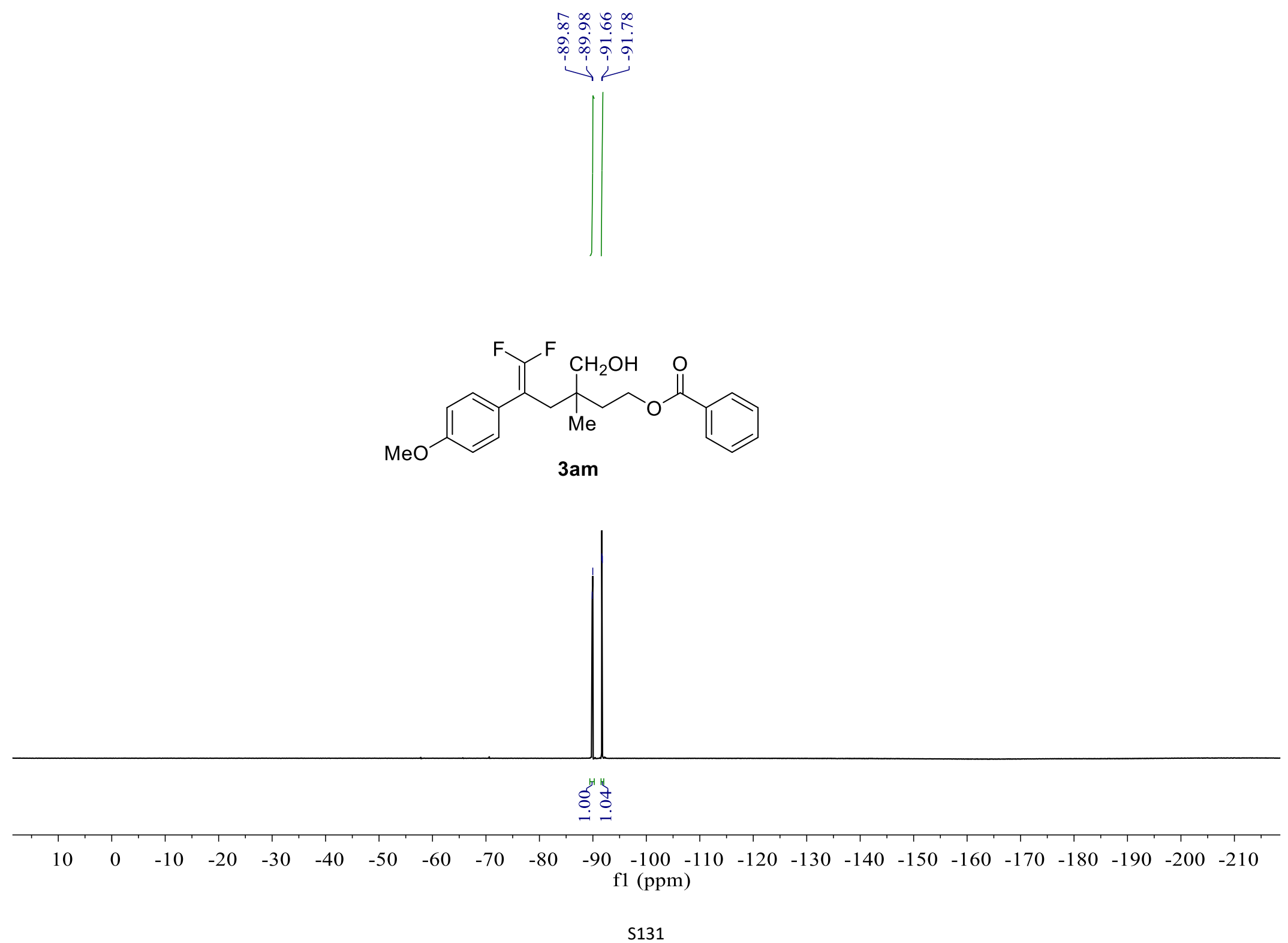
${ }^{1} \mathrm{H}$ NMR-spectrum $\left(400 \mathrm{MHz}, \mathrm{CDCl}_{3}\right)$ of 3an

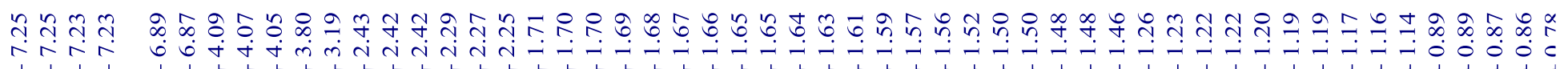
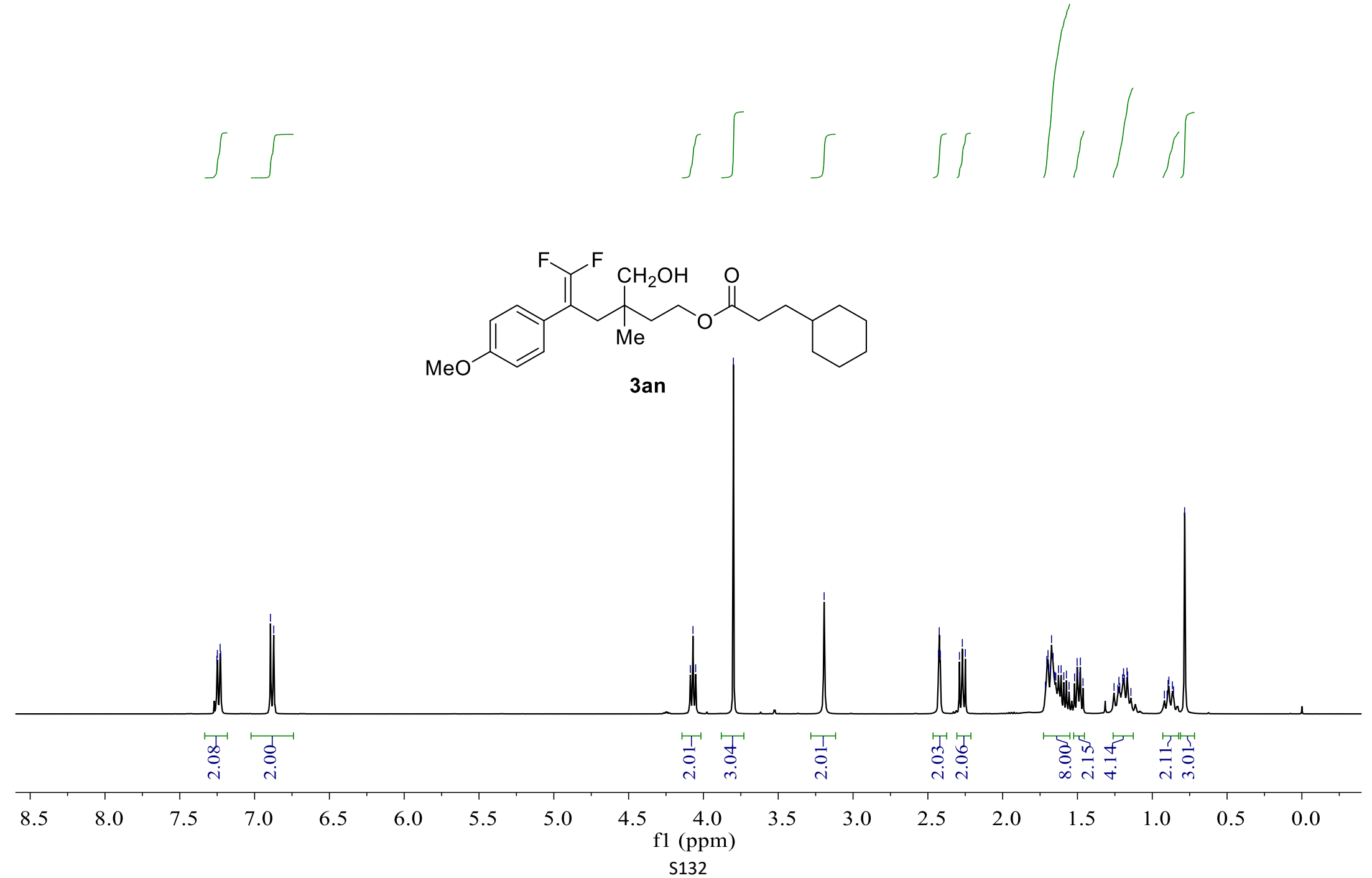
${ }^{13} \mathrm{C}$ NMR-spectrum $\left(101 \mathrm{MHz}, \mathrm{CDCl}_{3}\right)$ of 3an
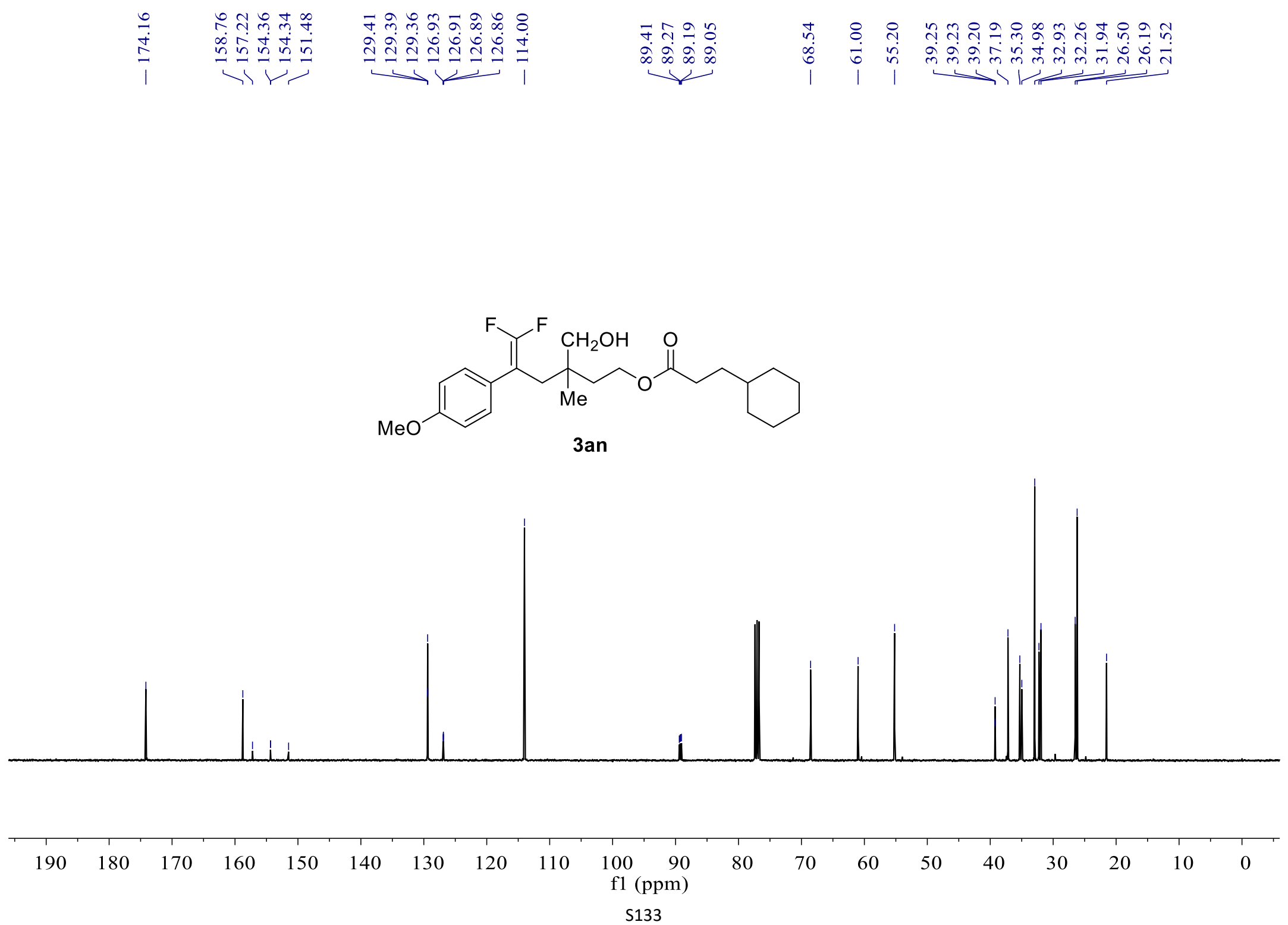
${ }^{19} \mathrm{~F}$ NMR-spectrum $\left(376 \mathrm{MHz}, \mathrm{CDCl}_{3}\right)$ of 3an
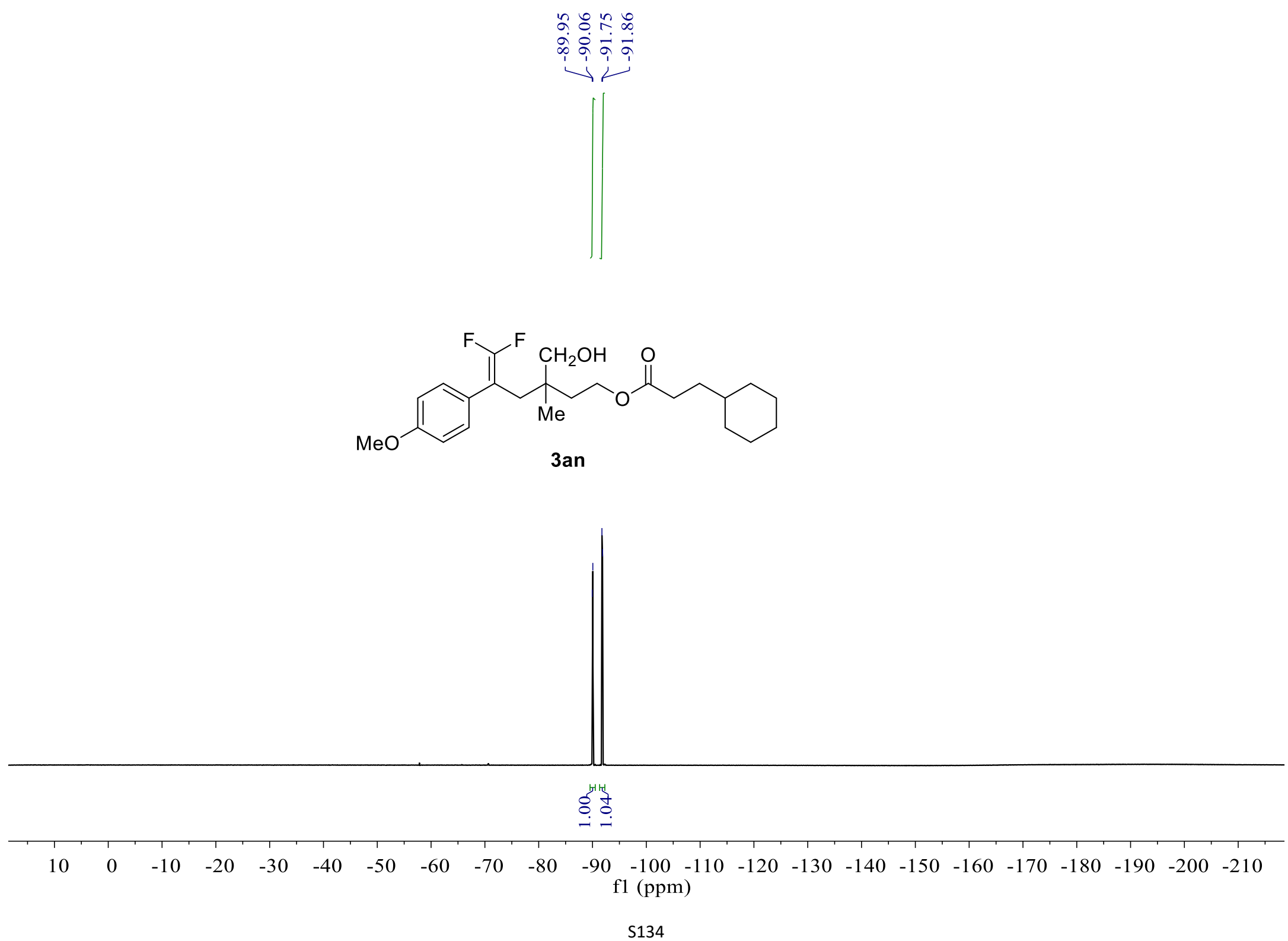
${ }^{1} \mathrm{H}$ NMR-spectrum $\left(400 \mathrm{MHz}, \mathrm{CDCl}_{3}\right)$ of $\mathbf{3 a o}$
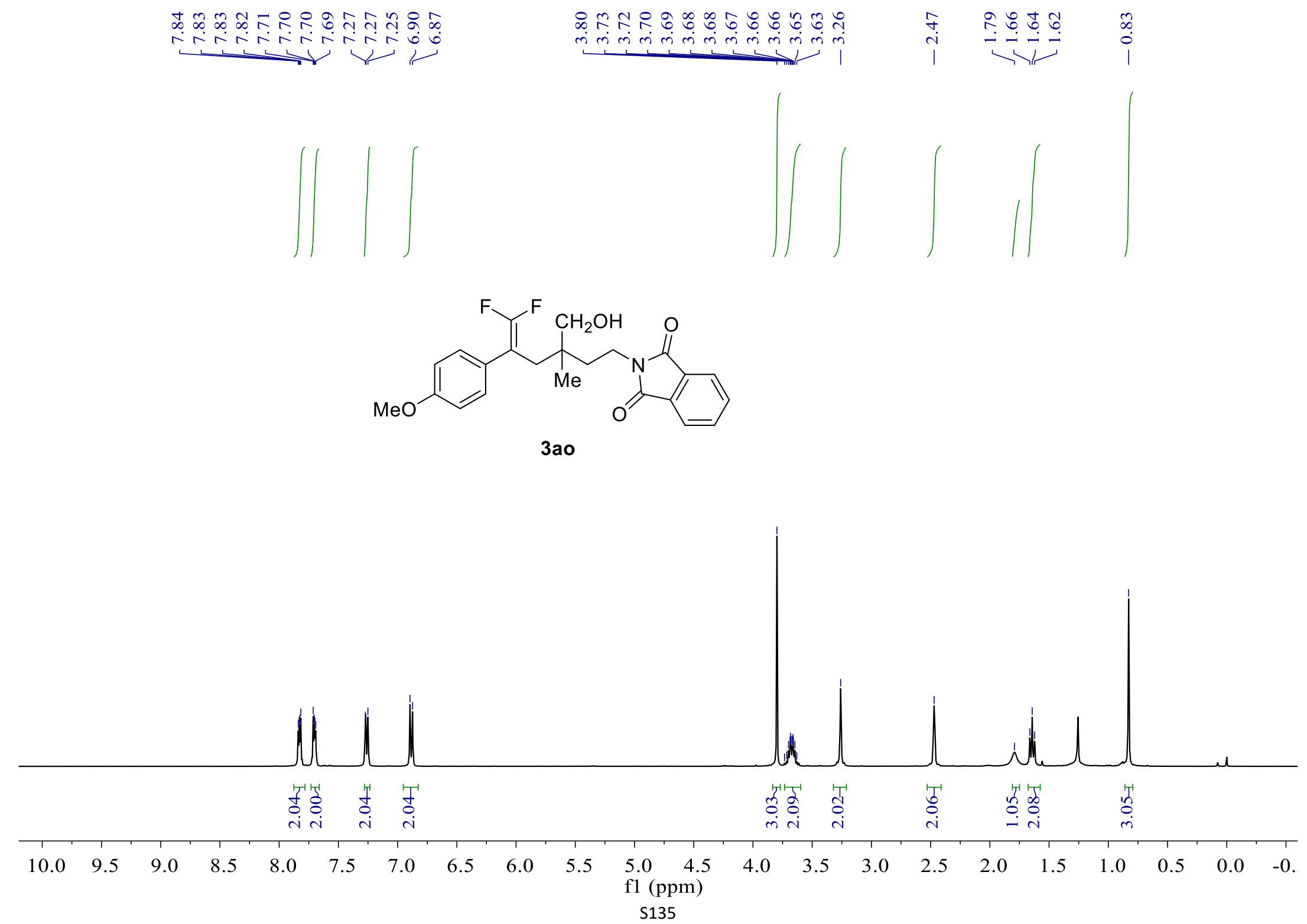
${ }^{13} \mathrm{C}$ NMR-spectrum (101 MHz, $\mathrm{CDCl}_{3}$ ) of 3ao

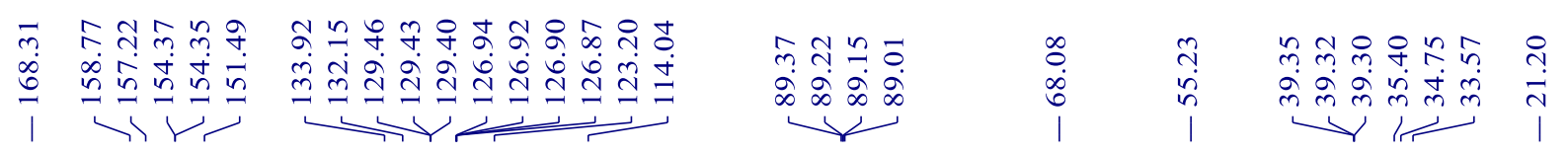

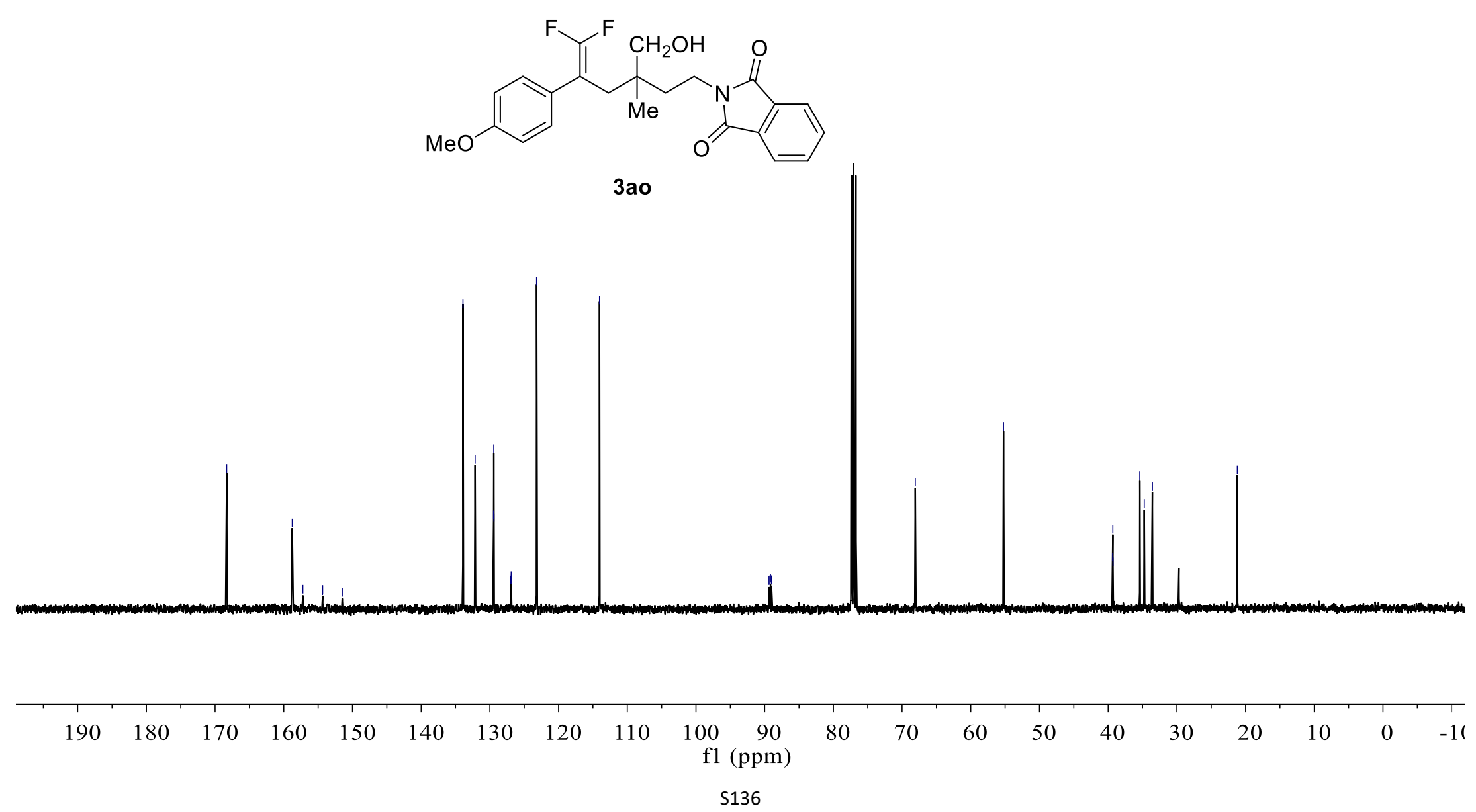


${ }^{19} \mathrm{~F} \mathrm{NMR}$-spectrum $\left(376 \mathrm{MHz}, \mathrm{CDCl}_{3}\right.$ ) of $\mathbf{3 a o}$ 


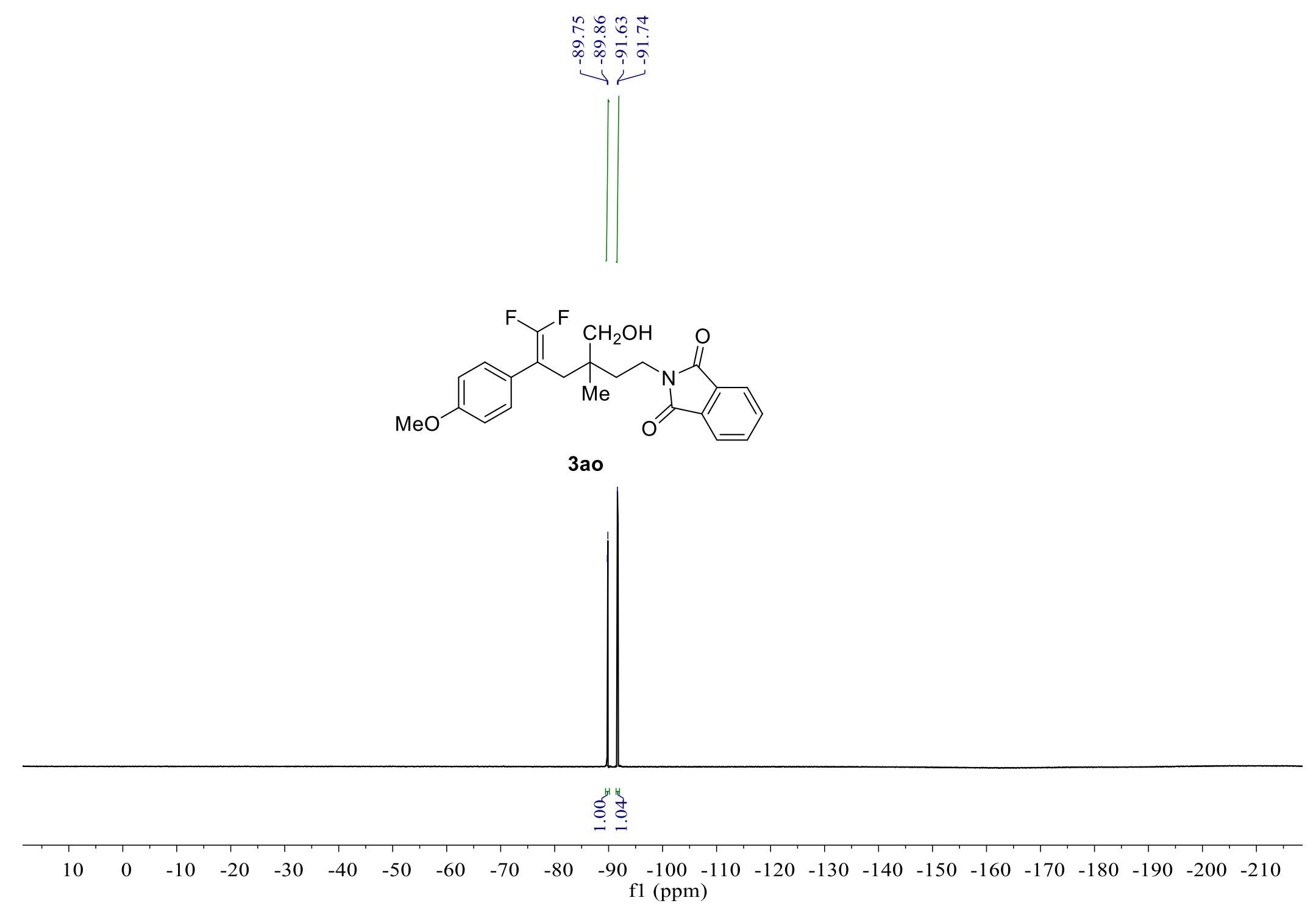

${ }^{1} \mathrm{H}$ NMR-spectrum (400 MHz, $\mathrm{CDCl}_{3}$ ) of 3ap 

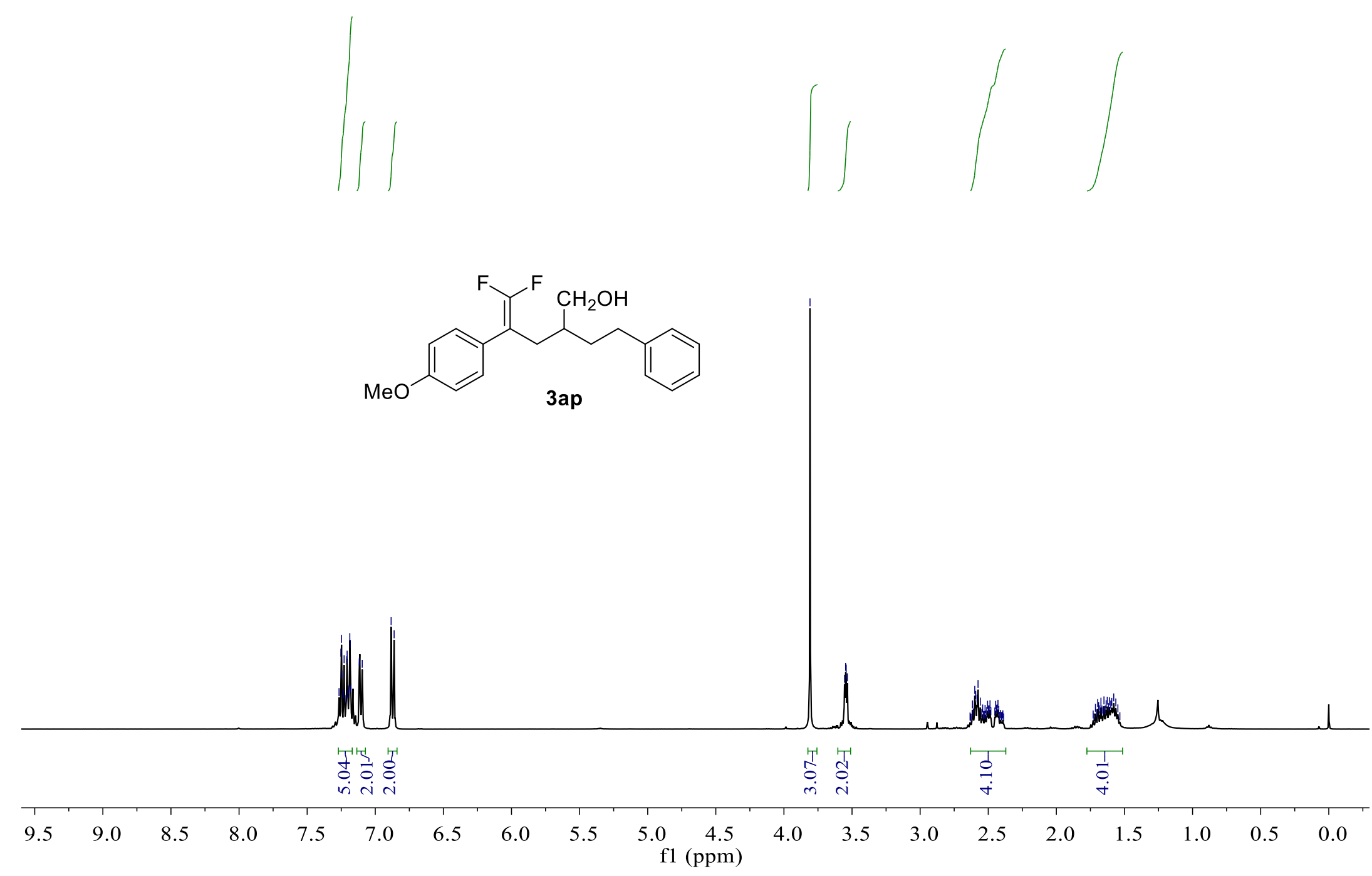

${ }^{13} \mathrm{C}$ NMR-spectrum $\left(101 \mathrm{MHz}, \mathrm{CDCl}_{3}\right)$ of 3ap 


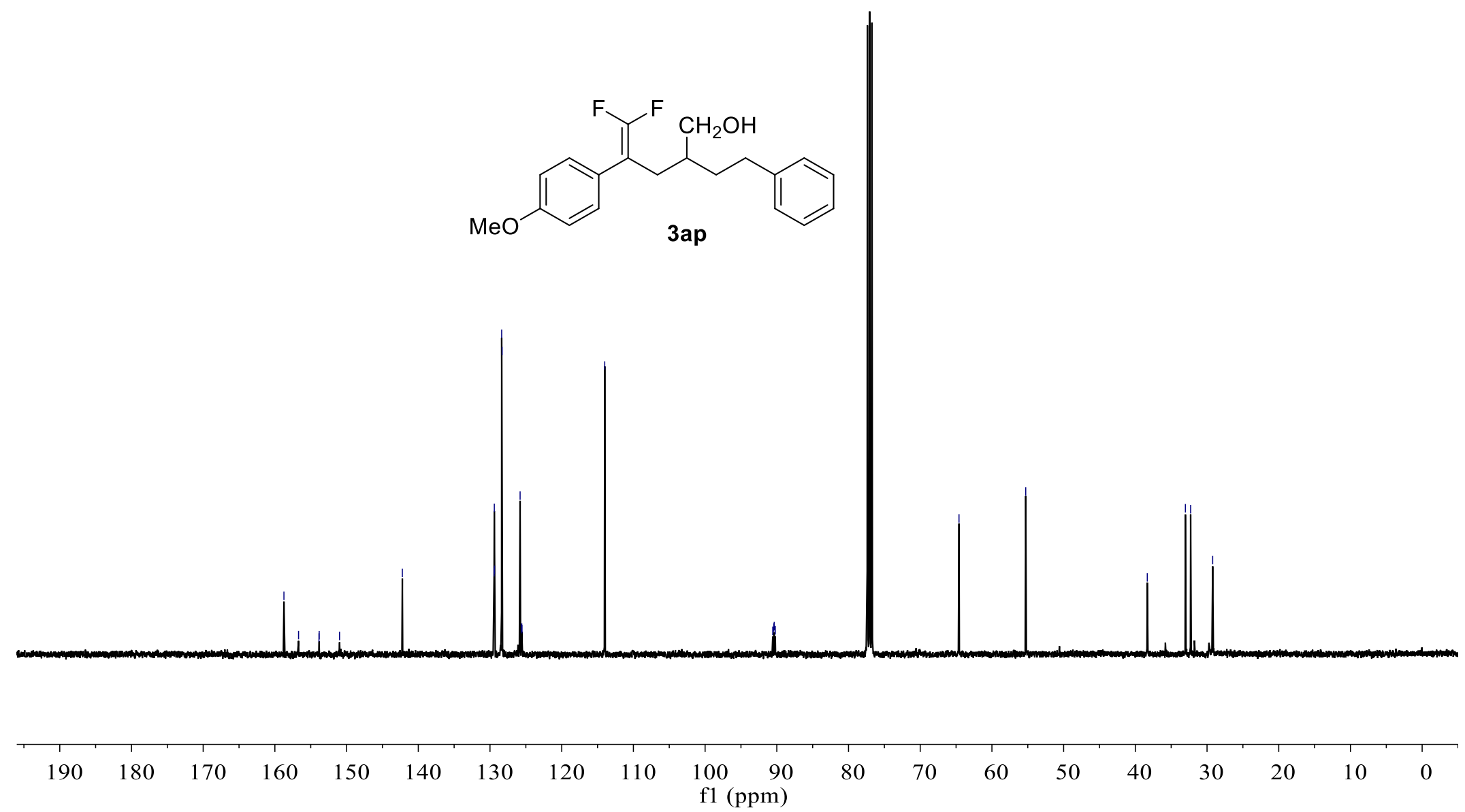

${ }^{19} \mathrm{~F}$ NMR-spectrum (376 MHz, $\mathrm{CDCl}_{3}$ ) of 3ap 


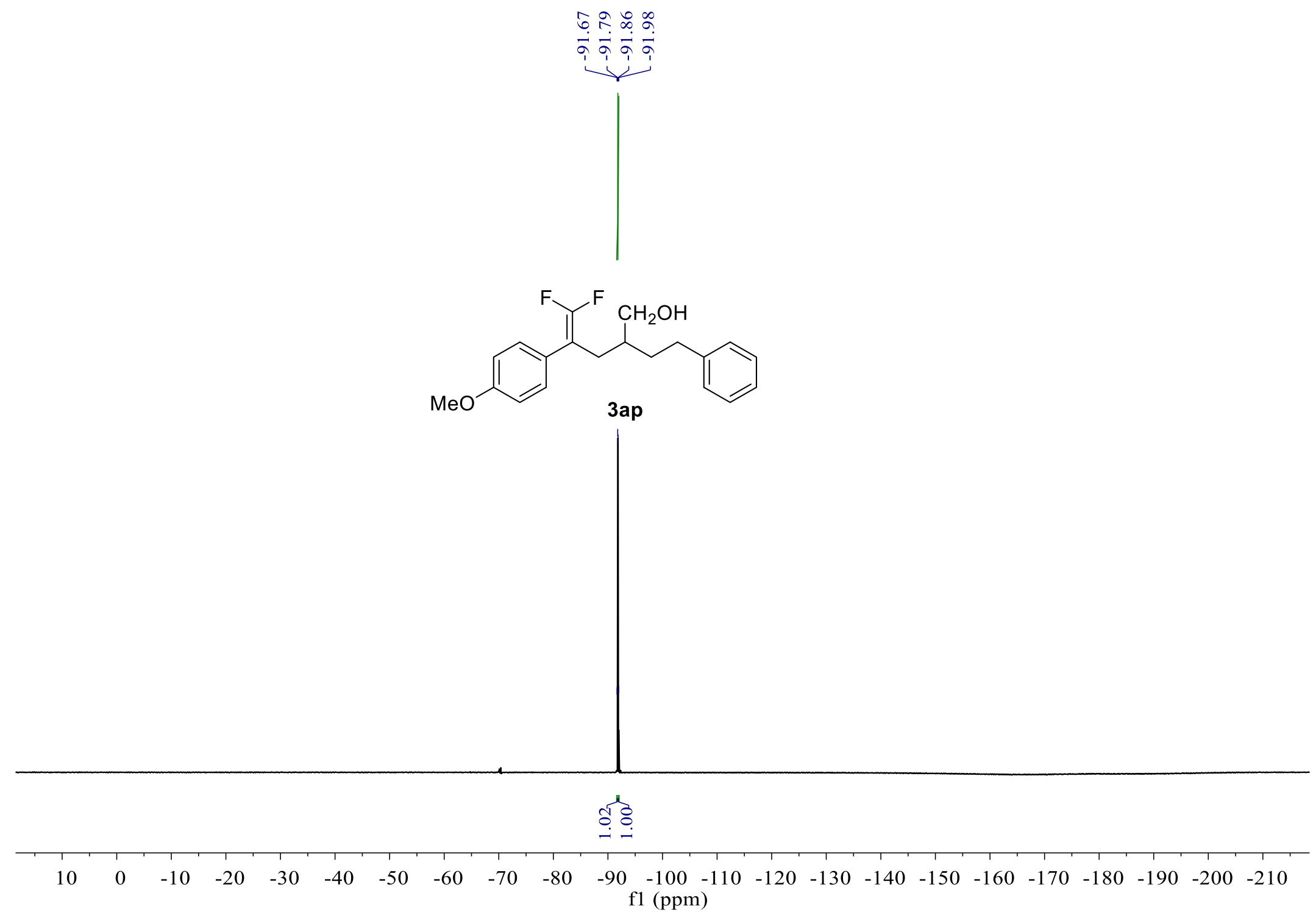

${ }^{1} \mathrm{H}$ NMR-spectrum $\left(500 \mathrm{MHz}, \mathrm{CDCl}_{3}\right)$ of $\mathbf{3 a q}$ 


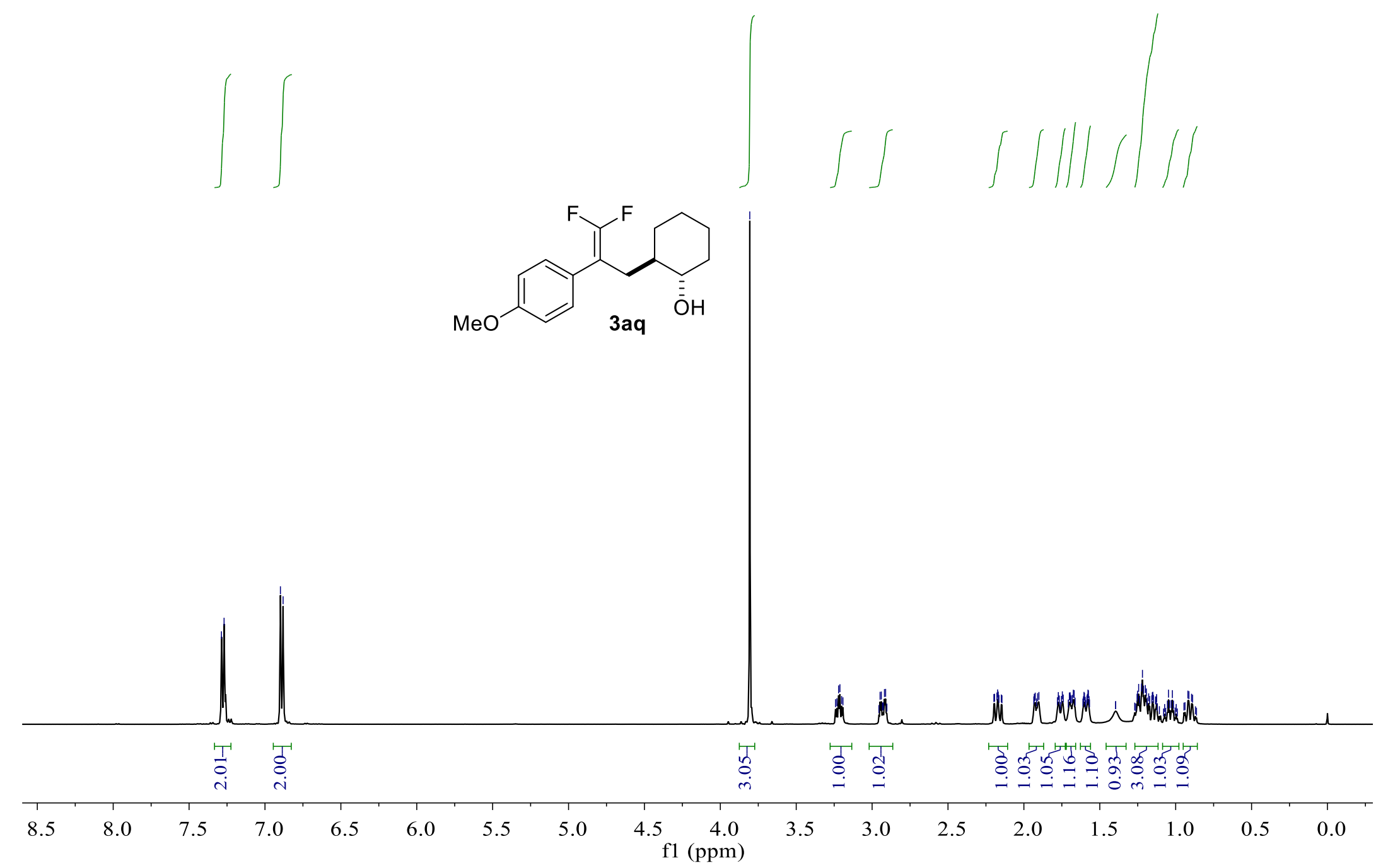

${ }^{13} \mathrm{C}$ NMR-spectrum $\left(126 \mathrm{MHz}, \mathrm{CDCl}_{3}\right)$ of $\mathbf{3 a q}$ 


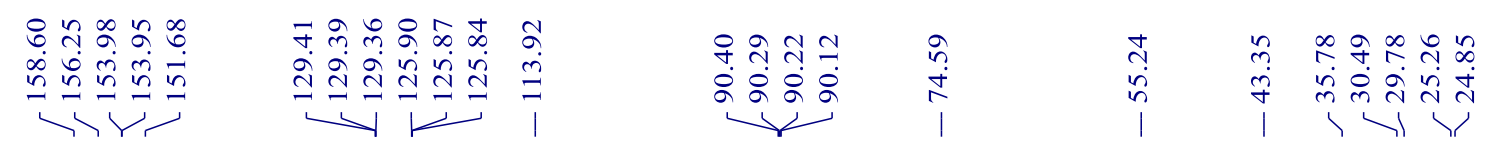
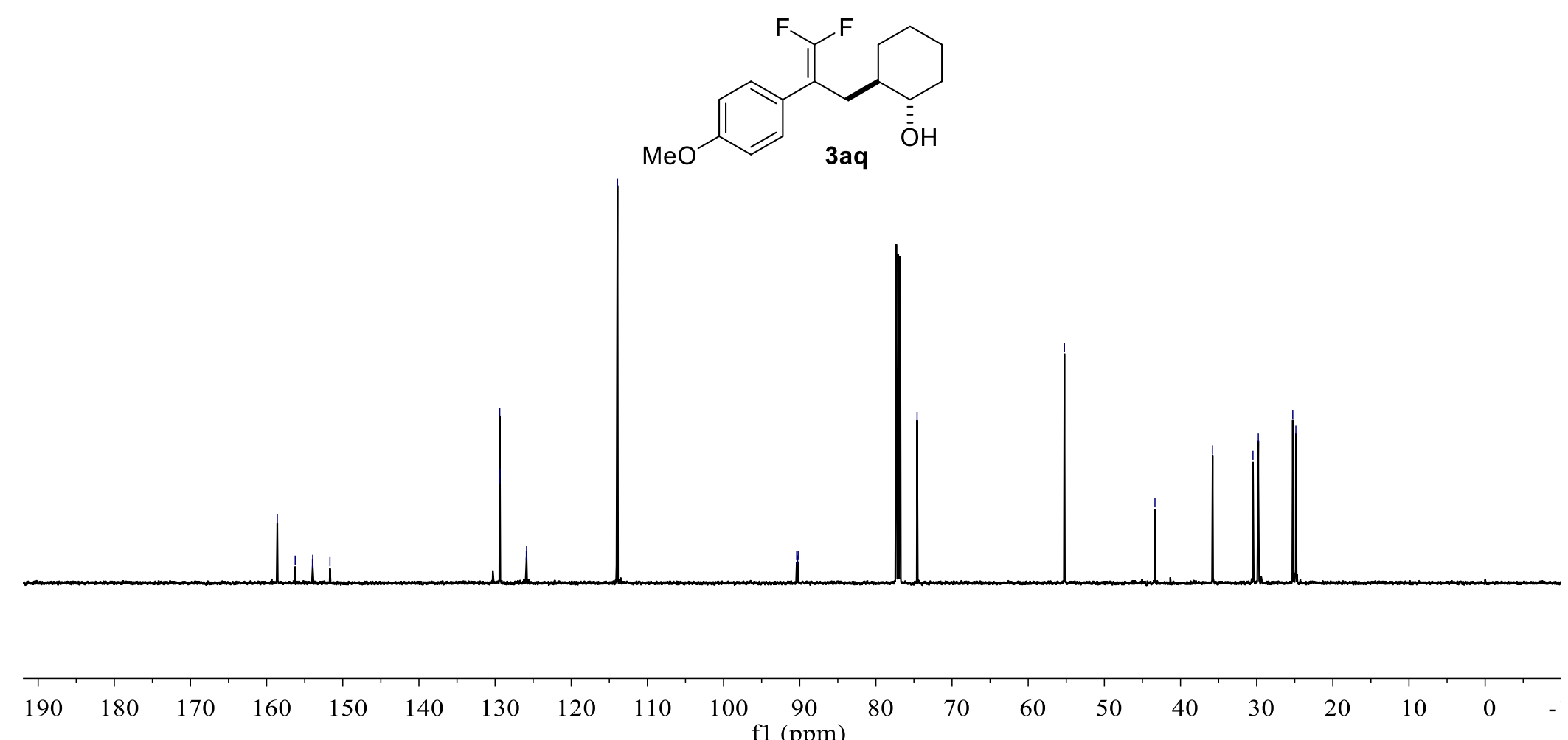

${ }^{19} \mathrm{~F}$ NMR-spectrum $\left(471 \mathrm{MHz}, \mathrm{CDCl}_{3}\right.$ ) of $\mathbf{3 a q}$ 


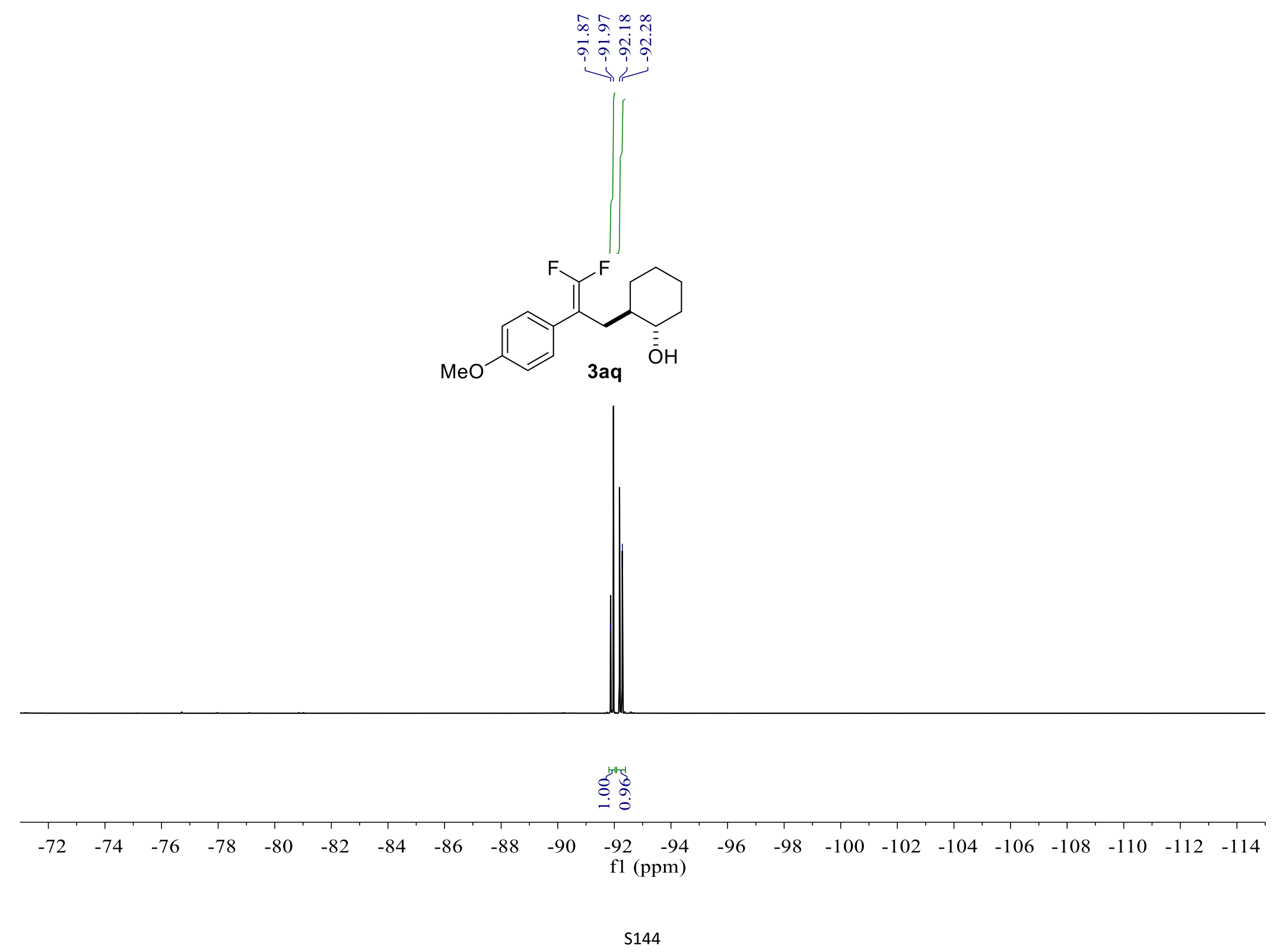



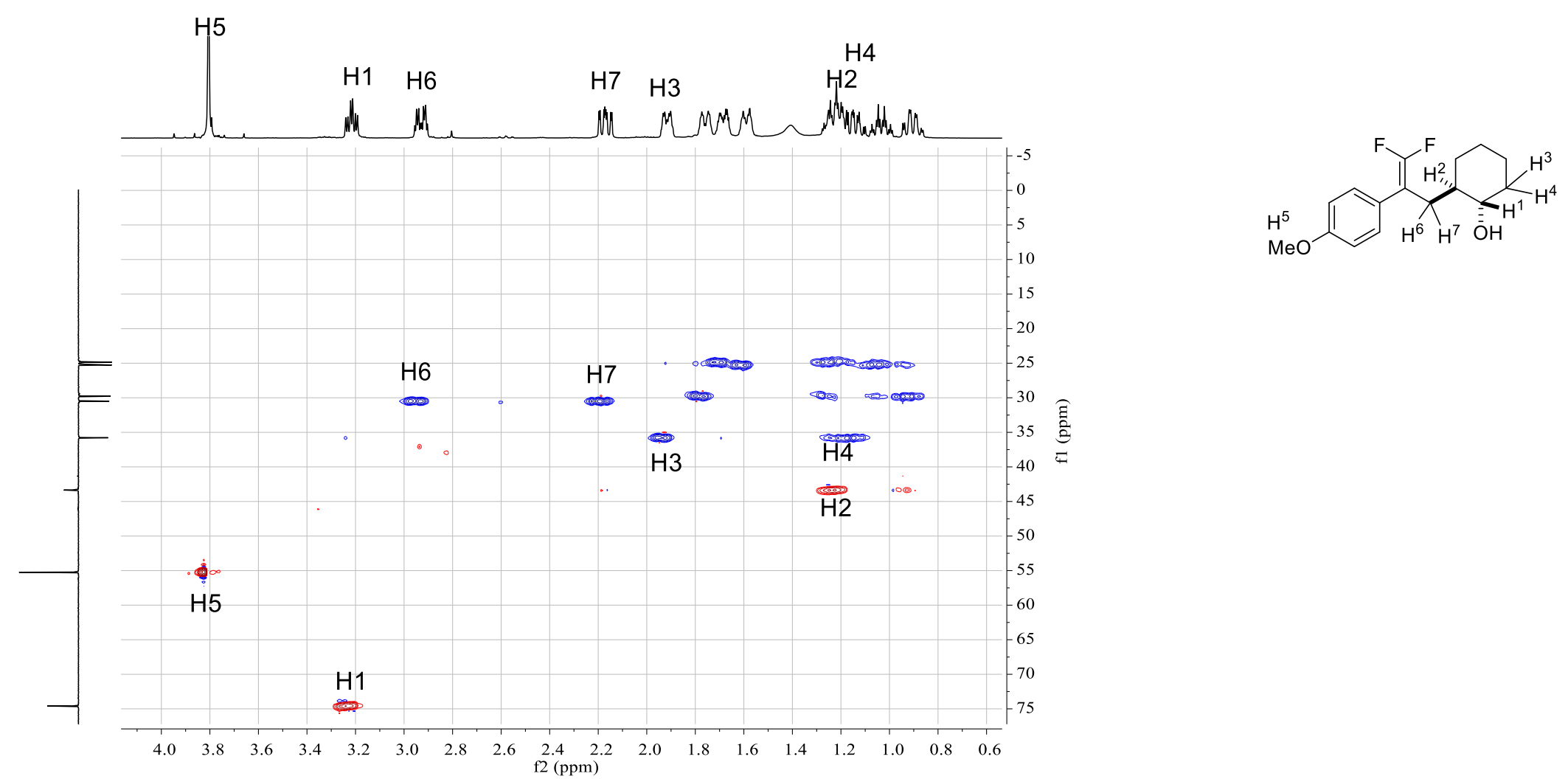

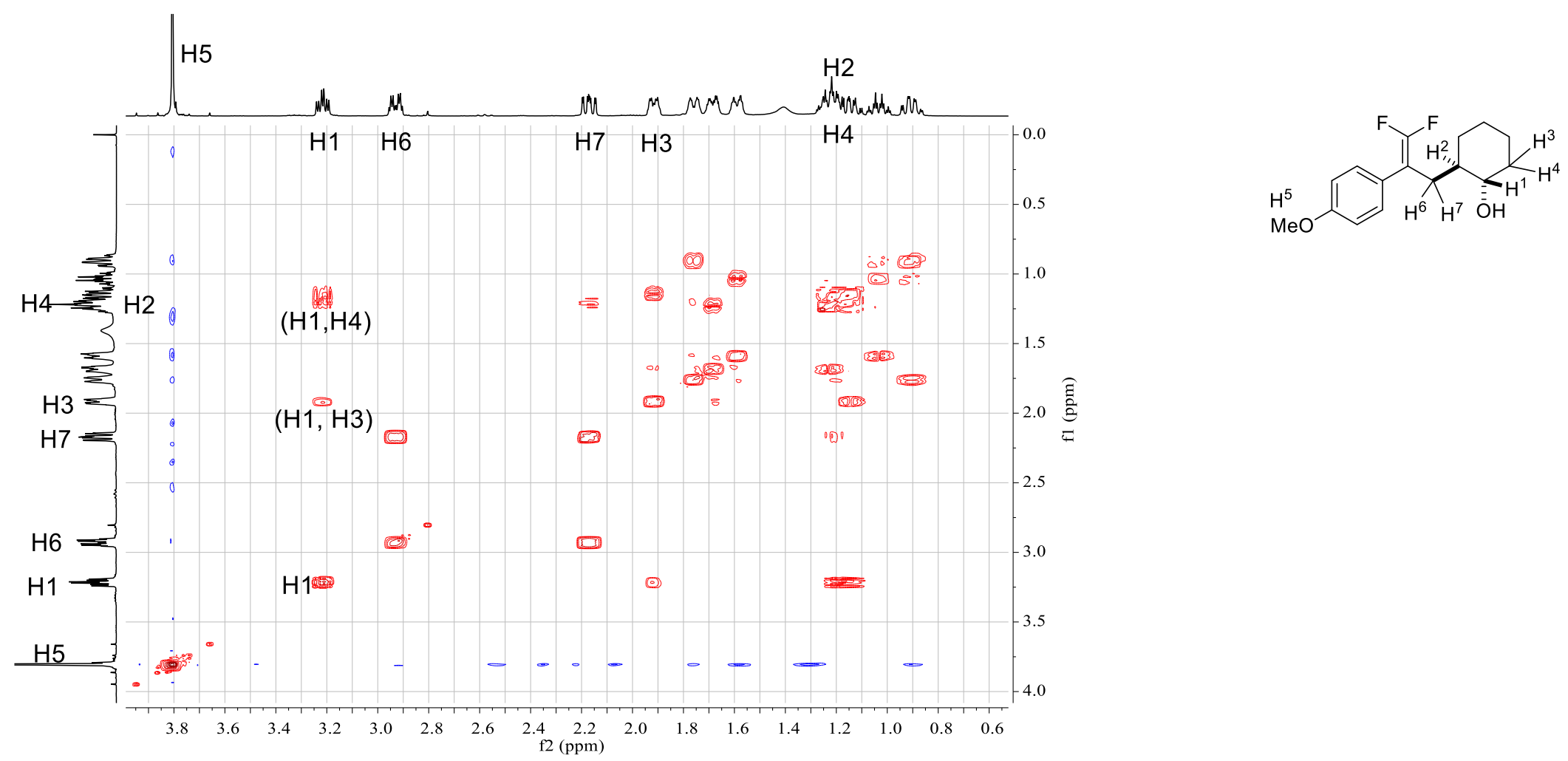

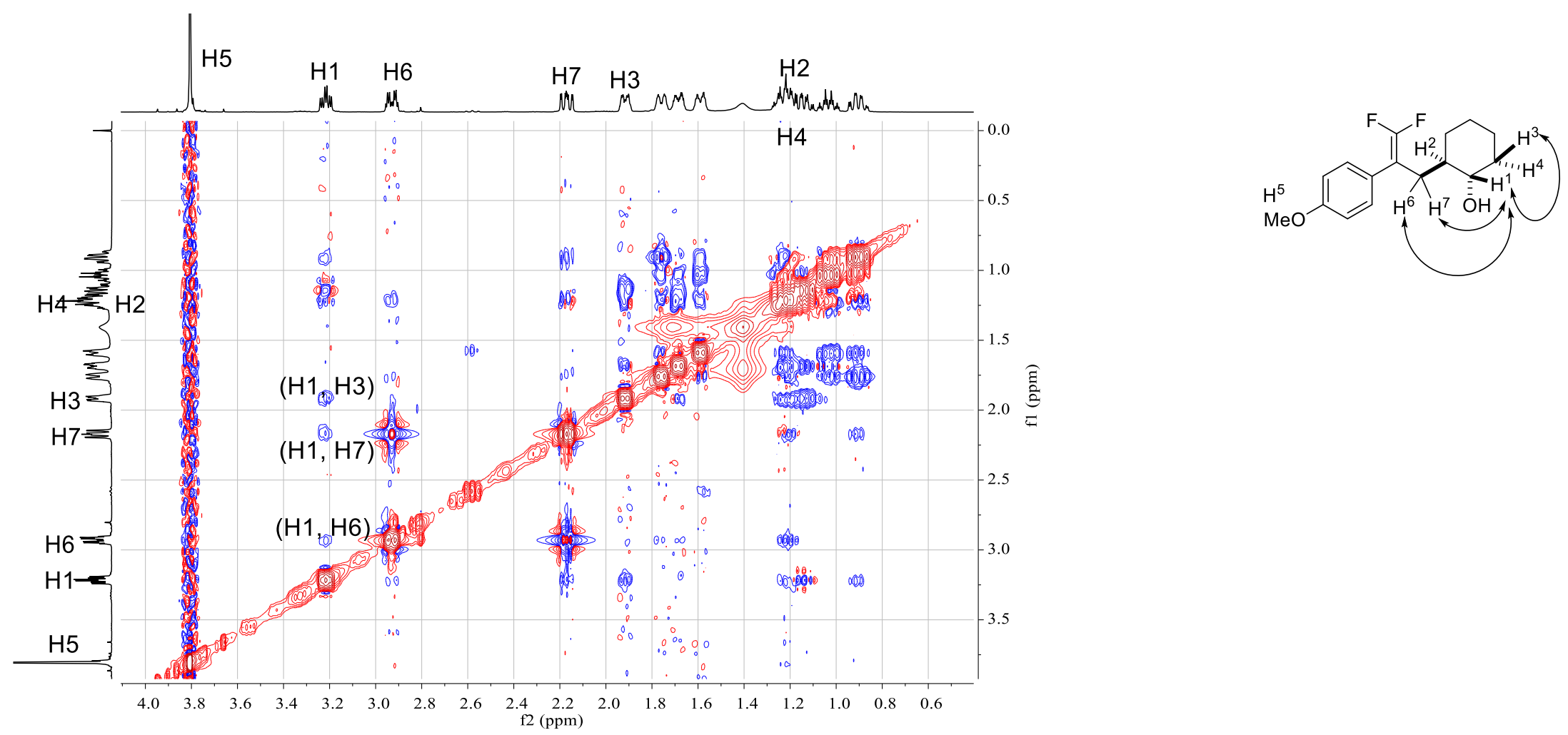

${ }^{1} \mathrm{H}$ NMR-spectrum $\left(500 \mathrm{MHz}, \mathrm{CDCl}_{3}\right.$ ) of $\mathbf{4 a a}$ 


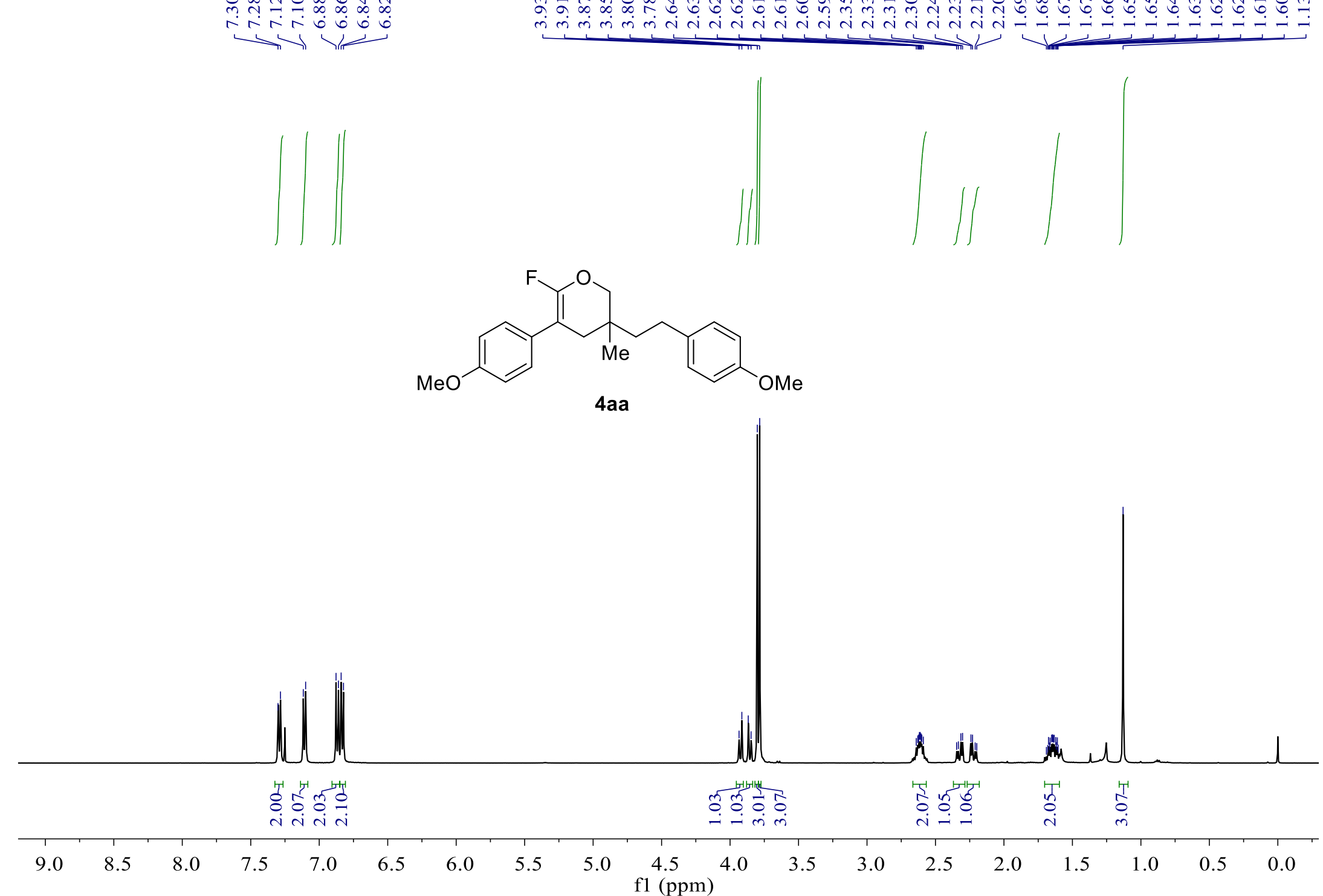


${ }^{13} \mathrm{C}$ NMR-spectrum $\left(126 \mathrm{MHz}, \mathrm{CDCl}_{3}\right)$ of $4 \mathbf{a a}$

\begin{tabular}{|c|c|c|c|c|c|}
\hline 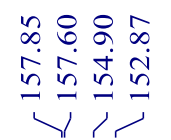 & 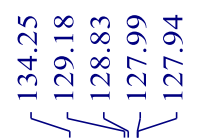 & $\begin{array}{l}8 \hat{\sigma} \\
\stackrel{m}{=} \\
=\end{array}$ & 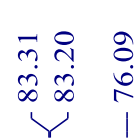 & $\begin{array}{c}\bar{n} \\
n \\
n \\
1\end{array}$ & 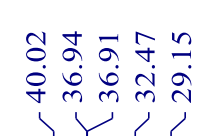 \\
\hline
\end{tabular}

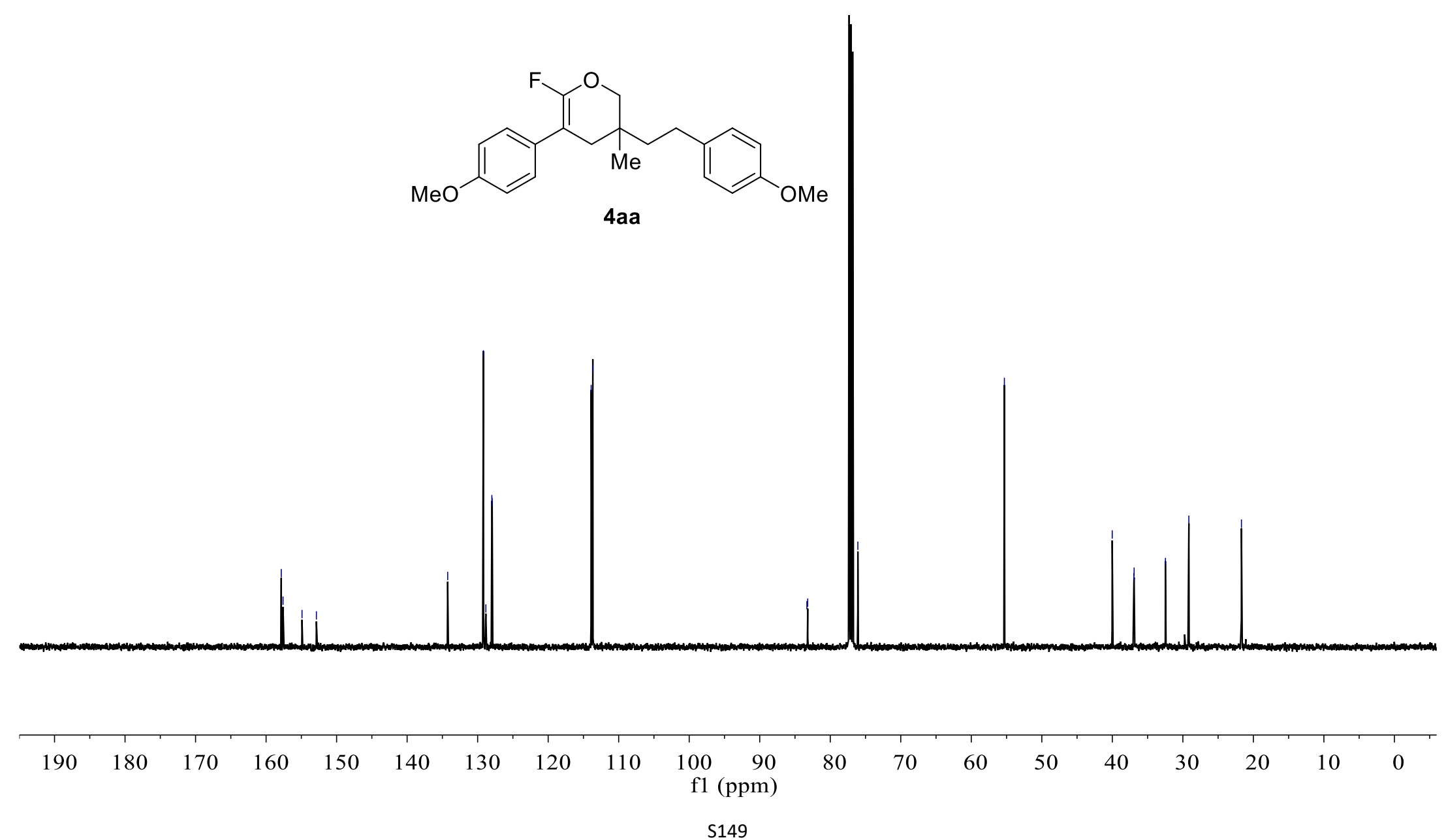


${ }^{19} \mathrm{~F}$ NMR-spectrum $\left(471 \mathrm{MHz}, \mathrm{CDCl}_{3}\right)$ of $\mathbf{4 a a}$

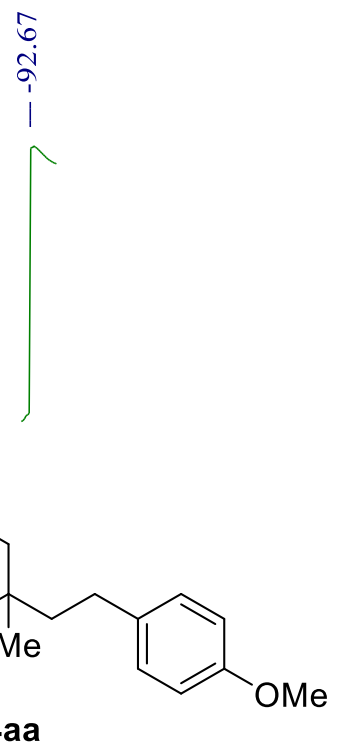

$4 a a$

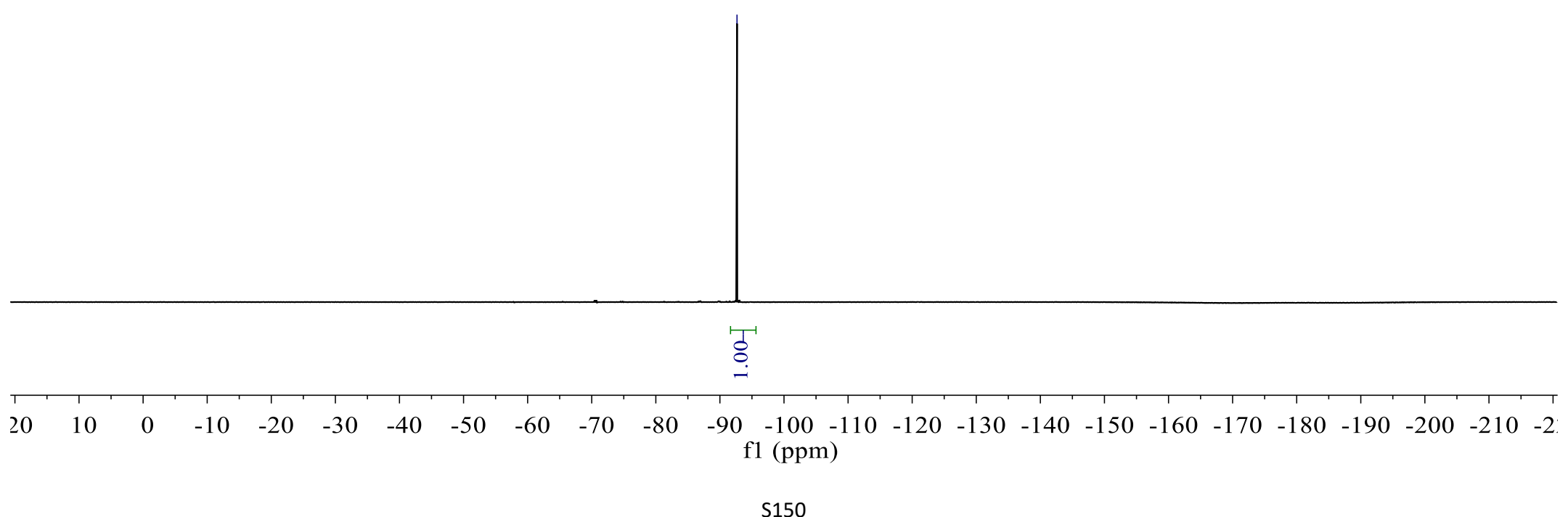


${ }^{1} \mathrm{H}$ NMR-spectrum $\left(400 \mathrm{MHz}, \mathrm{CDCl}_{3}\right.$ ) of $\mathbf{4 b a}$

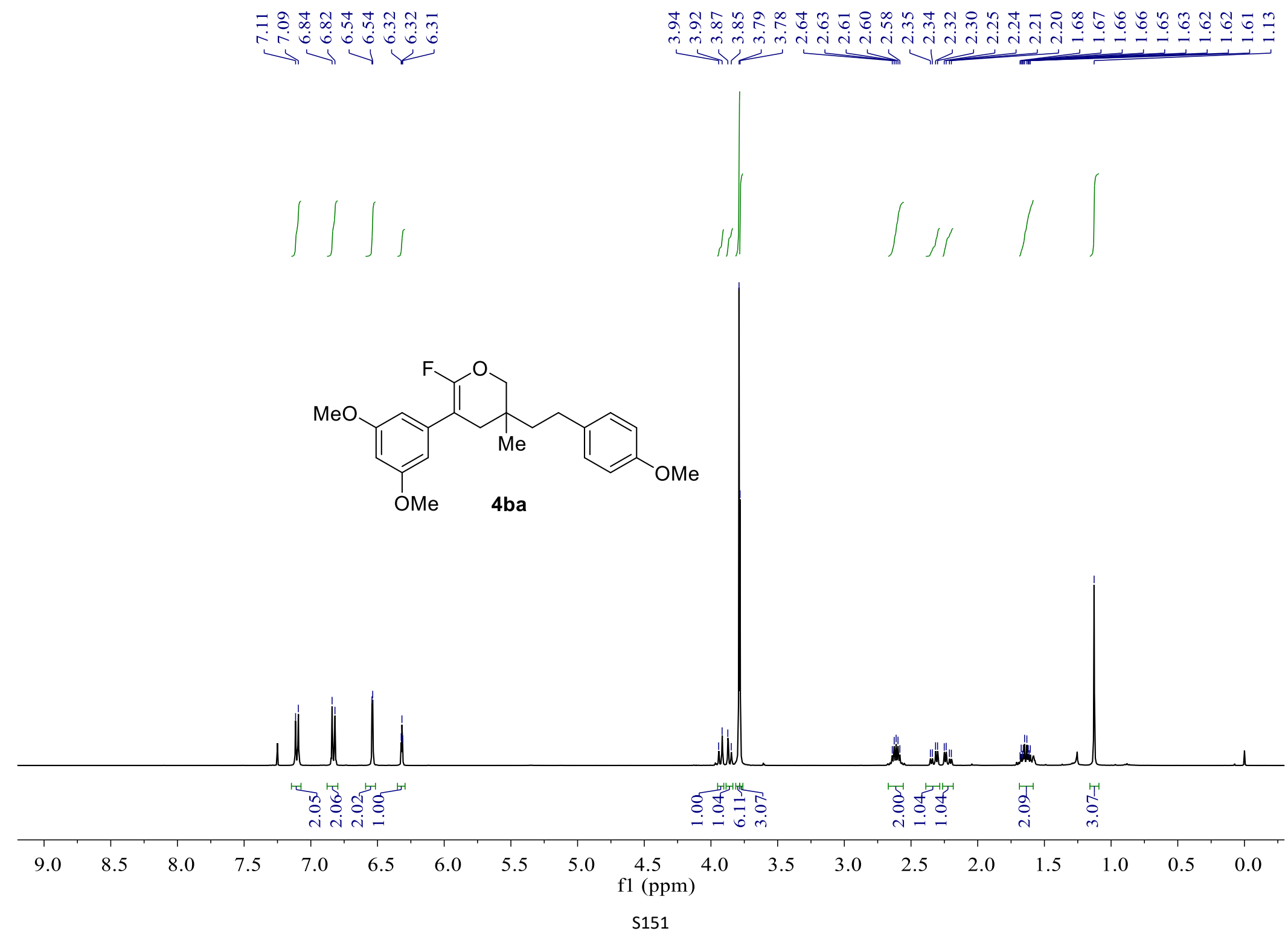


${ }^{13} \mathrm{C}$ NMR-spectrum (101 MHz, $\mathrm{CDCl}_{3}$ ) of $\mathbf{4 b a}$

\begin{tabular}{|c|c|c|c|c|c|c|c|c|}
\hline 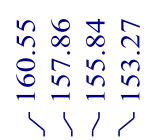 & 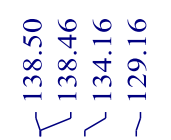 & $\vec{a}$ & 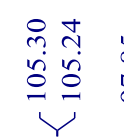 & $\begin{array}{l}\infty \\
\infty \\
a \\
a \\
1\end{array}$ & 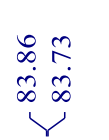 & $\frac{2}{6}$ & $\hat{n}_{\substack{n \\
n}}^{n}$ & 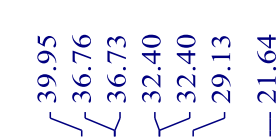 \\
\hline
\end{tabular}

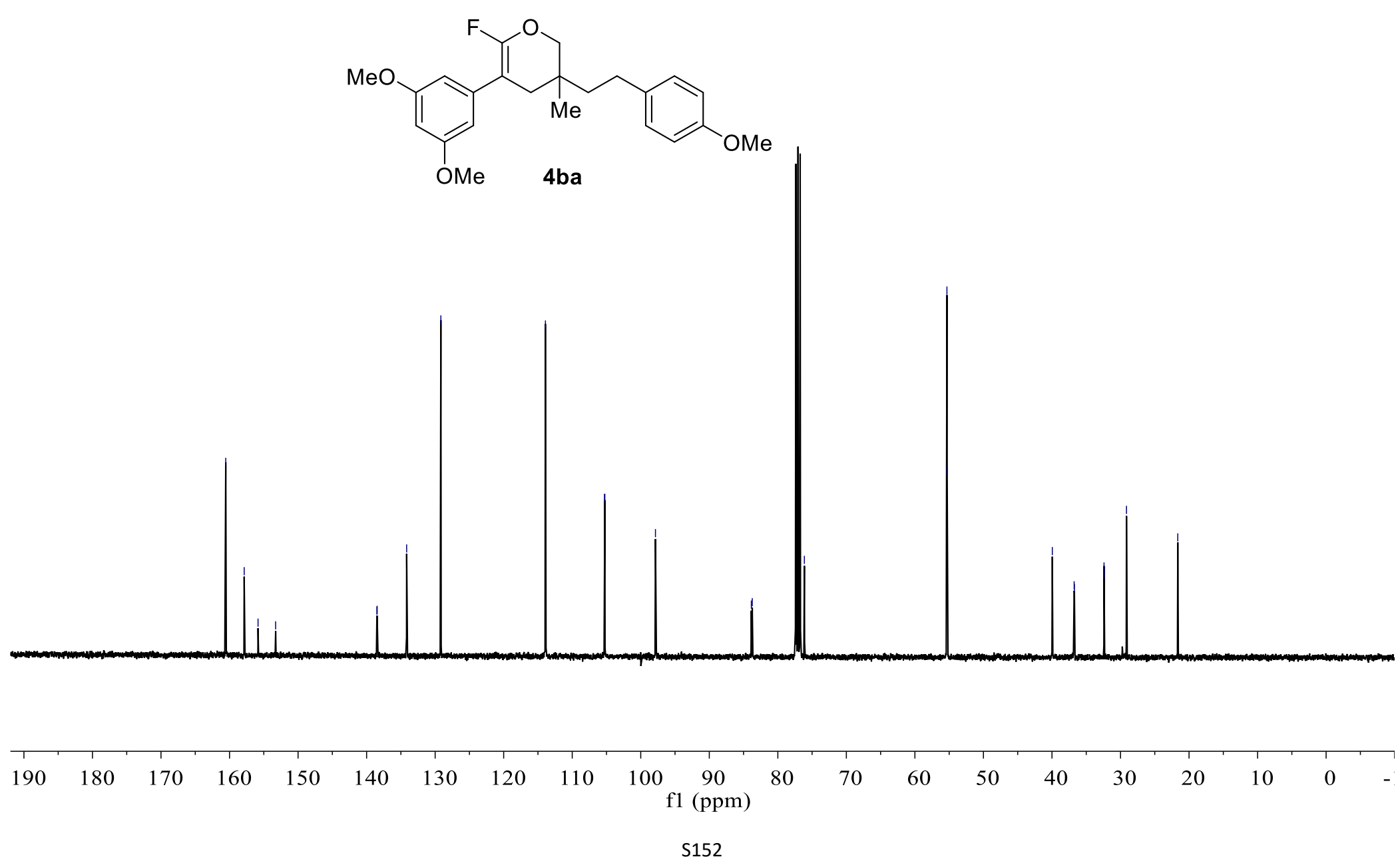


${ }^{19} \mathrm{~F}$ NMR-spectrum $\left(376 \mathrm{MHz}, \mathrm{CDCl}_{3}\right)$ of $\mathbf{4 b a}$

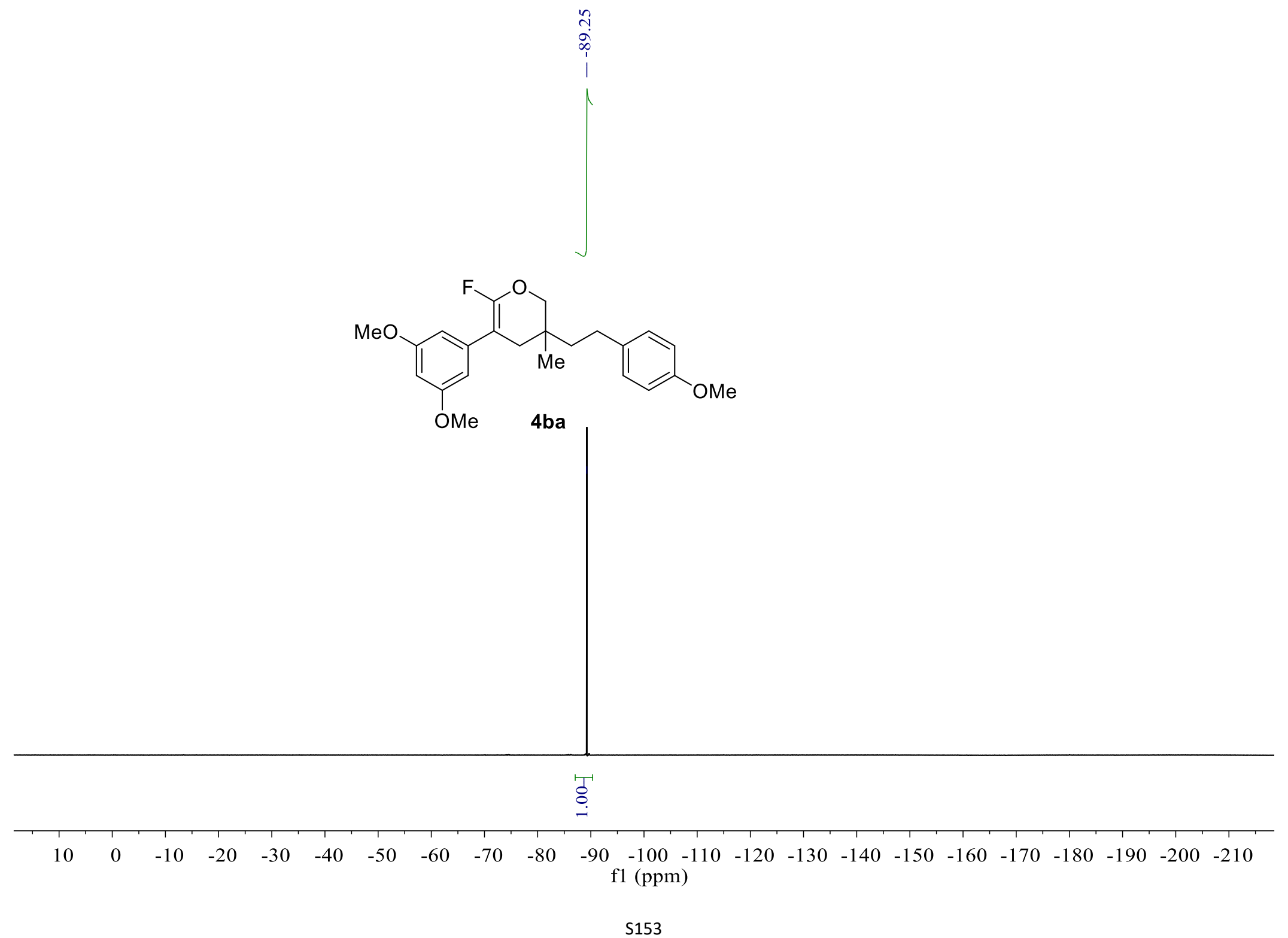


${ }^{1} \mathrm{H}$ NMR-spectrum $\left(400 \mathrm{MHz}, \mathrm{CDCl}_{3}\right)$ of $\mathbf{4} \mathbf{c a}$
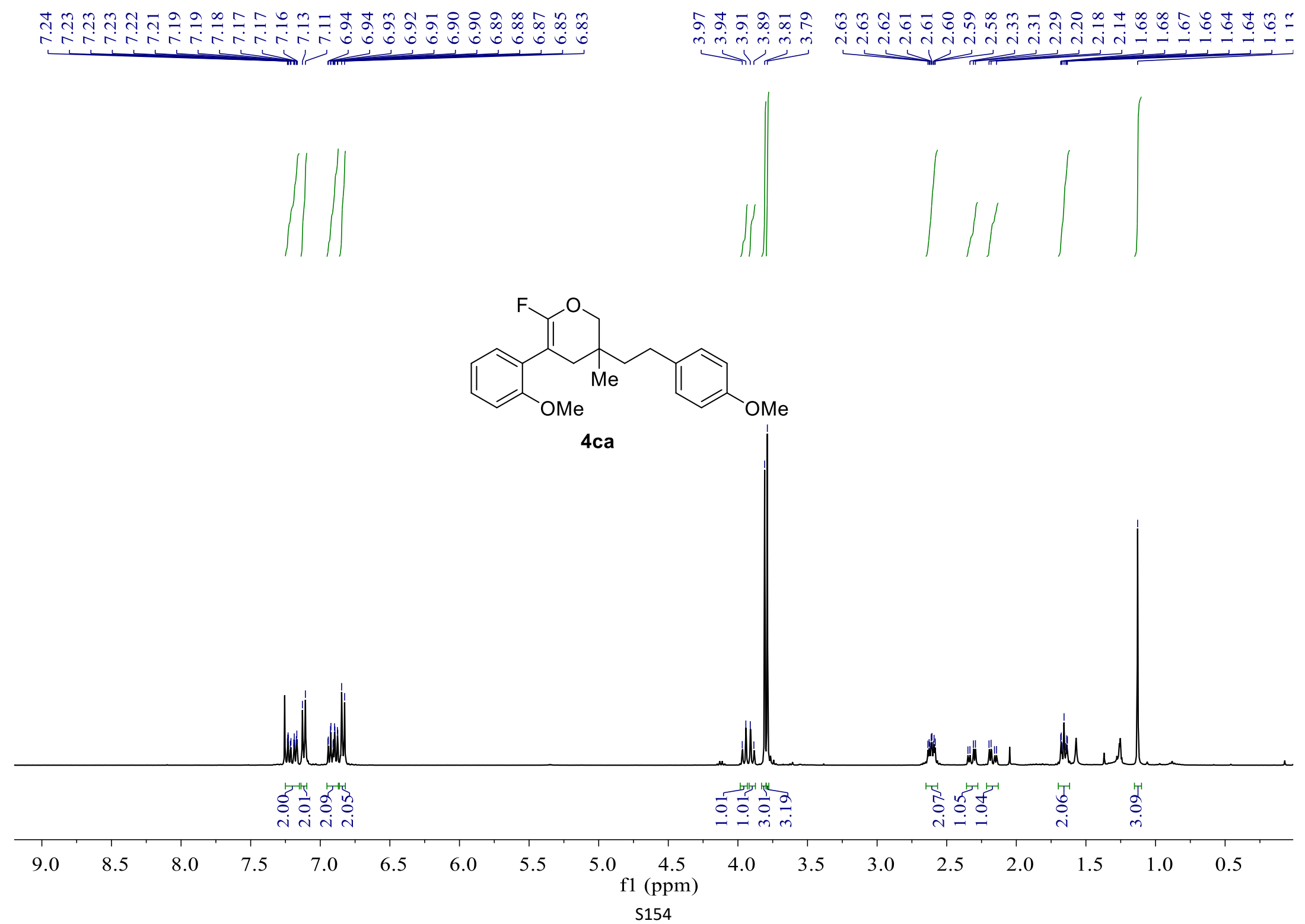
${ }^{13} \mathrm{C}$ NMR-spectrum $\left(101 \mathrm{MHz}, \mathrm{CDCl}_{3}\right)$ of $\mathbf{4 c a}$

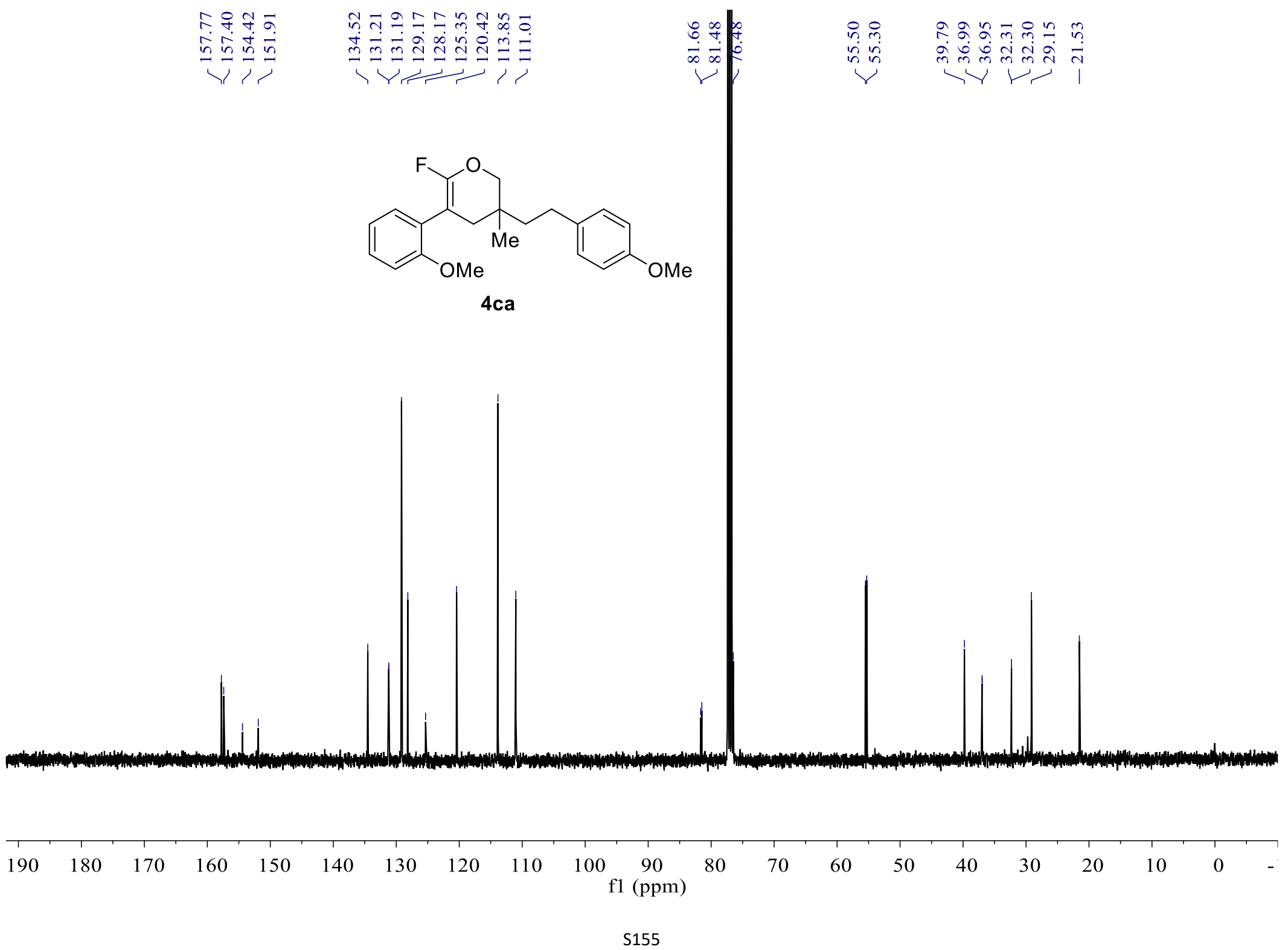


${ }^{19} \mathrm{~F}$ NMR-spectrum $\left(376 \mathrm{MHz}, \mathrm{CDCl}_{3}\right.$ ) of $\mathbf{4} \mathbf{c a}$

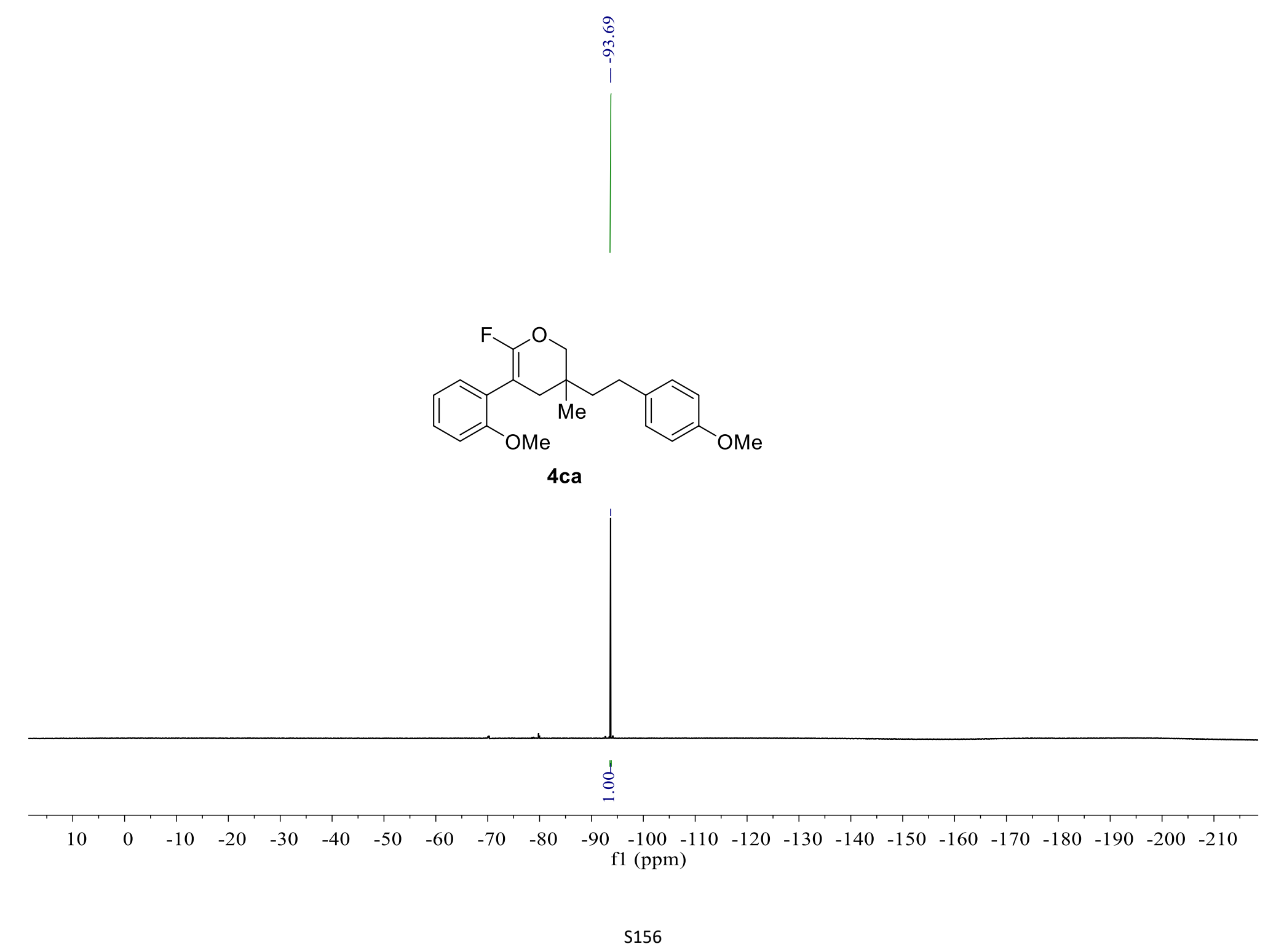



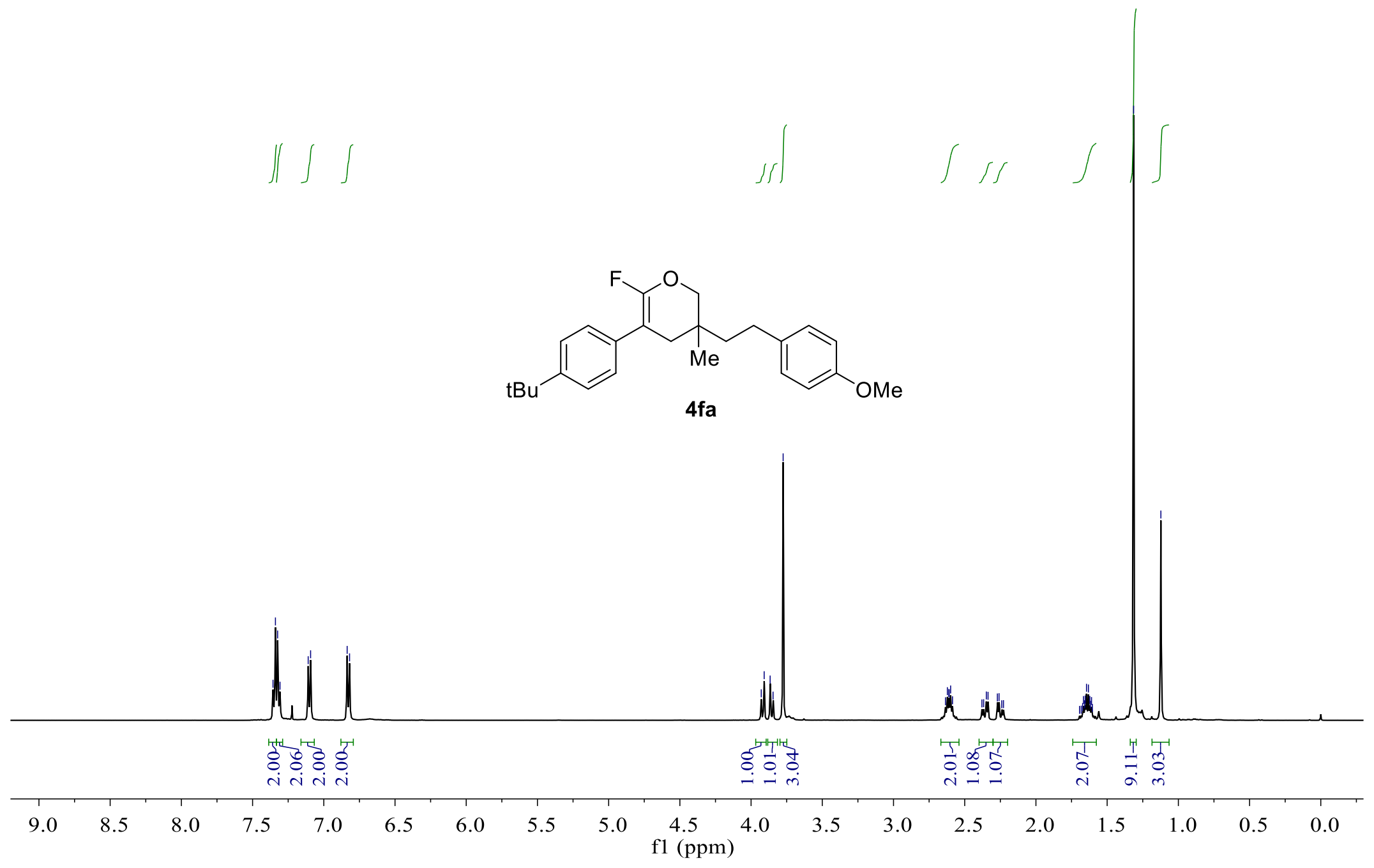
${ }^{13} \mathrm{C}$ NMR-spectrum $\left(126 \mathrm{MHz}, \mathrm{CDCl}_{3}\right)$ of $\mathbf{4 f a}$
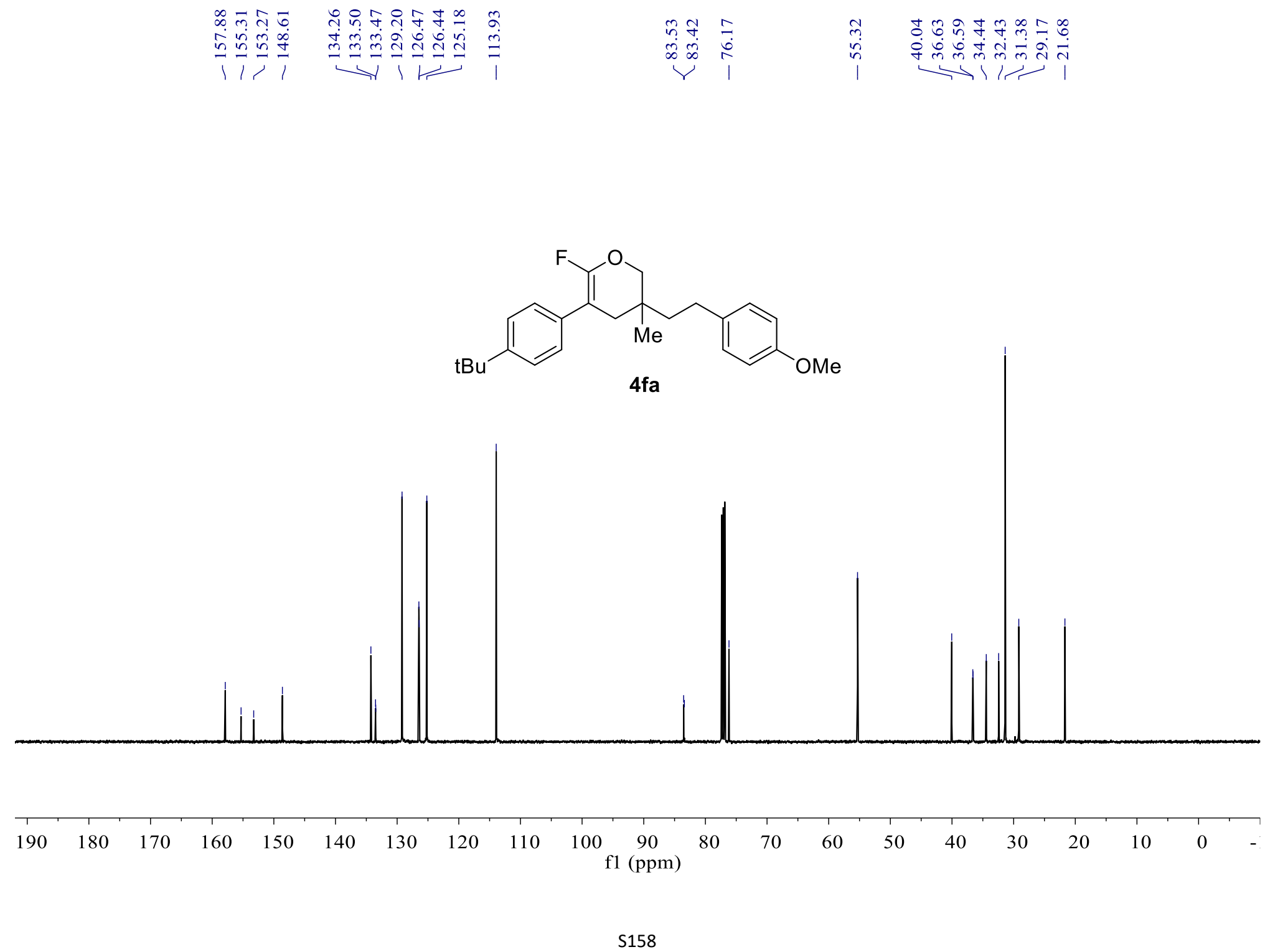
${ }^{19} \mathrm{~F}$ NMR-spectrum $\left(471 \mathrm{MHz}, \mathrm{CDCl}_{3}\right)$ of $\mathbf{4 f a}$

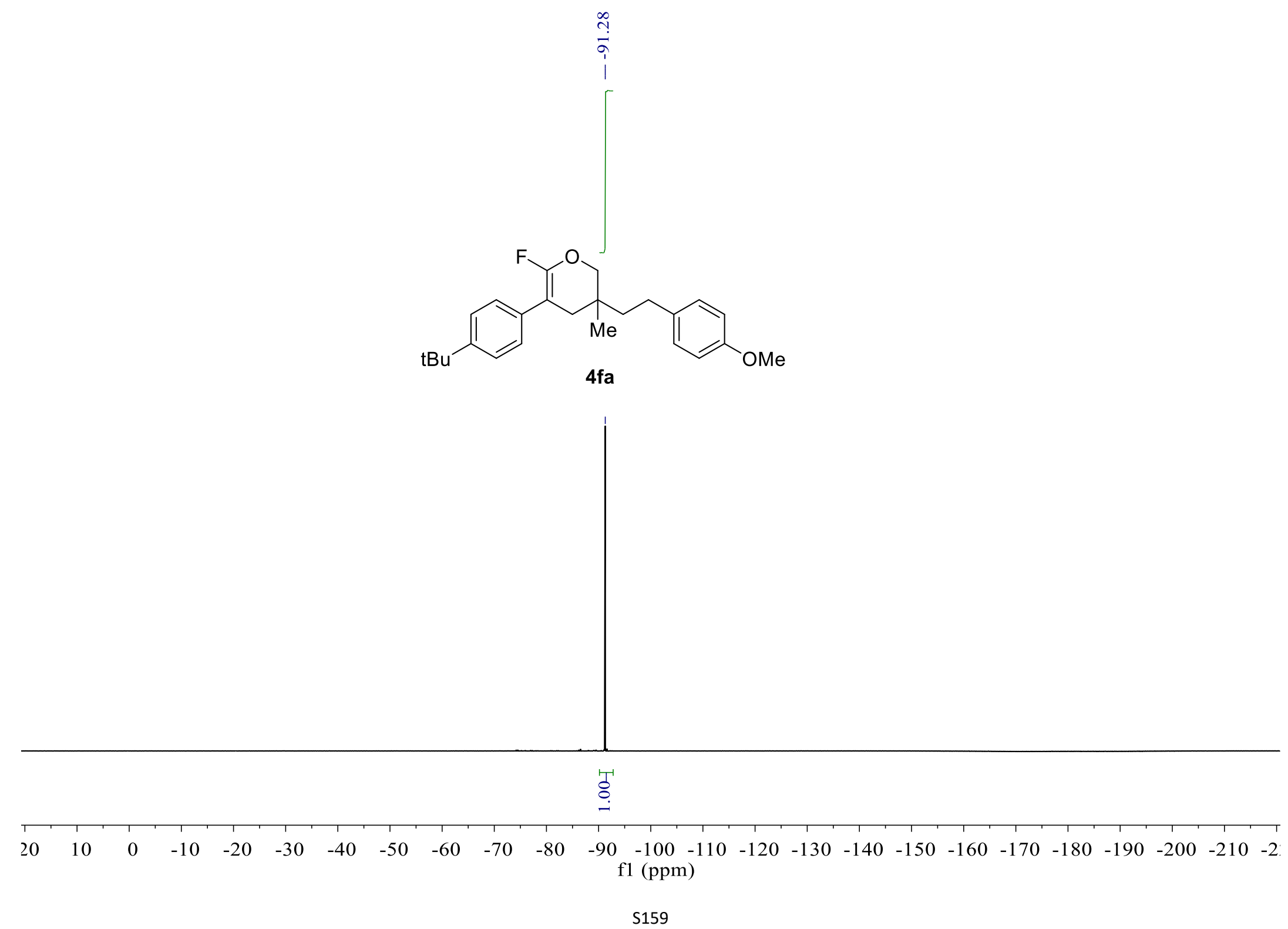


${ }^{1} \mathrm{H}$ NMR-spectrum $\left(500 \mathrm{MHz}, \mathrm{CDCl}_{3}\right)$ of 4 ha

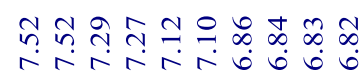
年

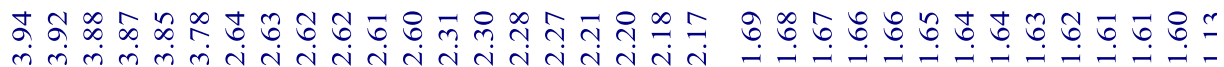
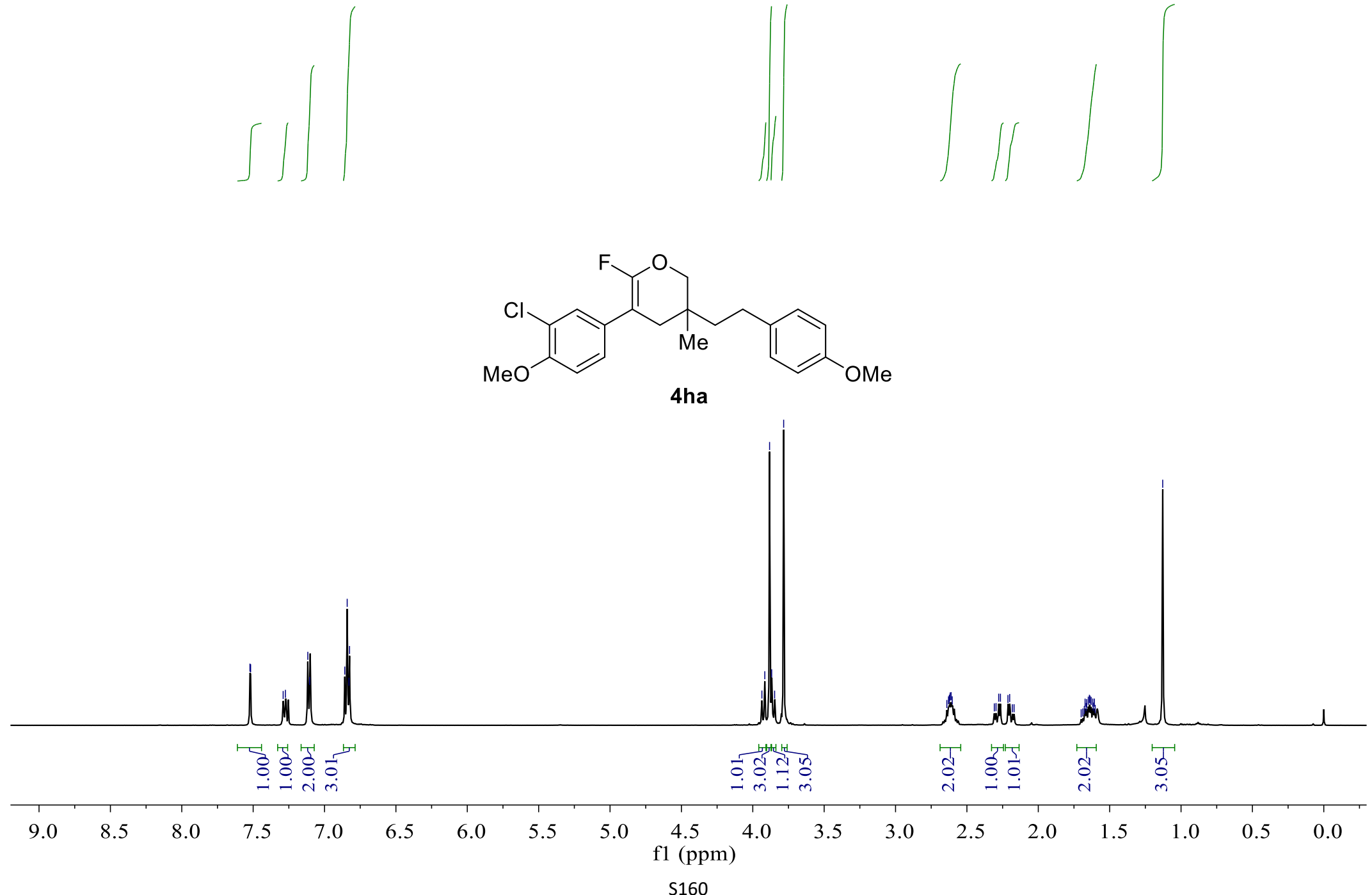
${ }^{13} \mathrm{C}$ NMR-spectrum $\left(126 \mathrm{MHz}, \mathrm{CDCl}_{3}\right)$ of 4 ha

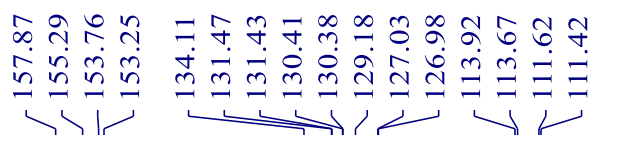

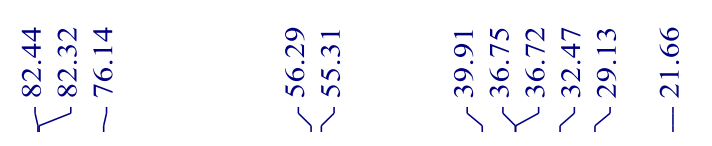

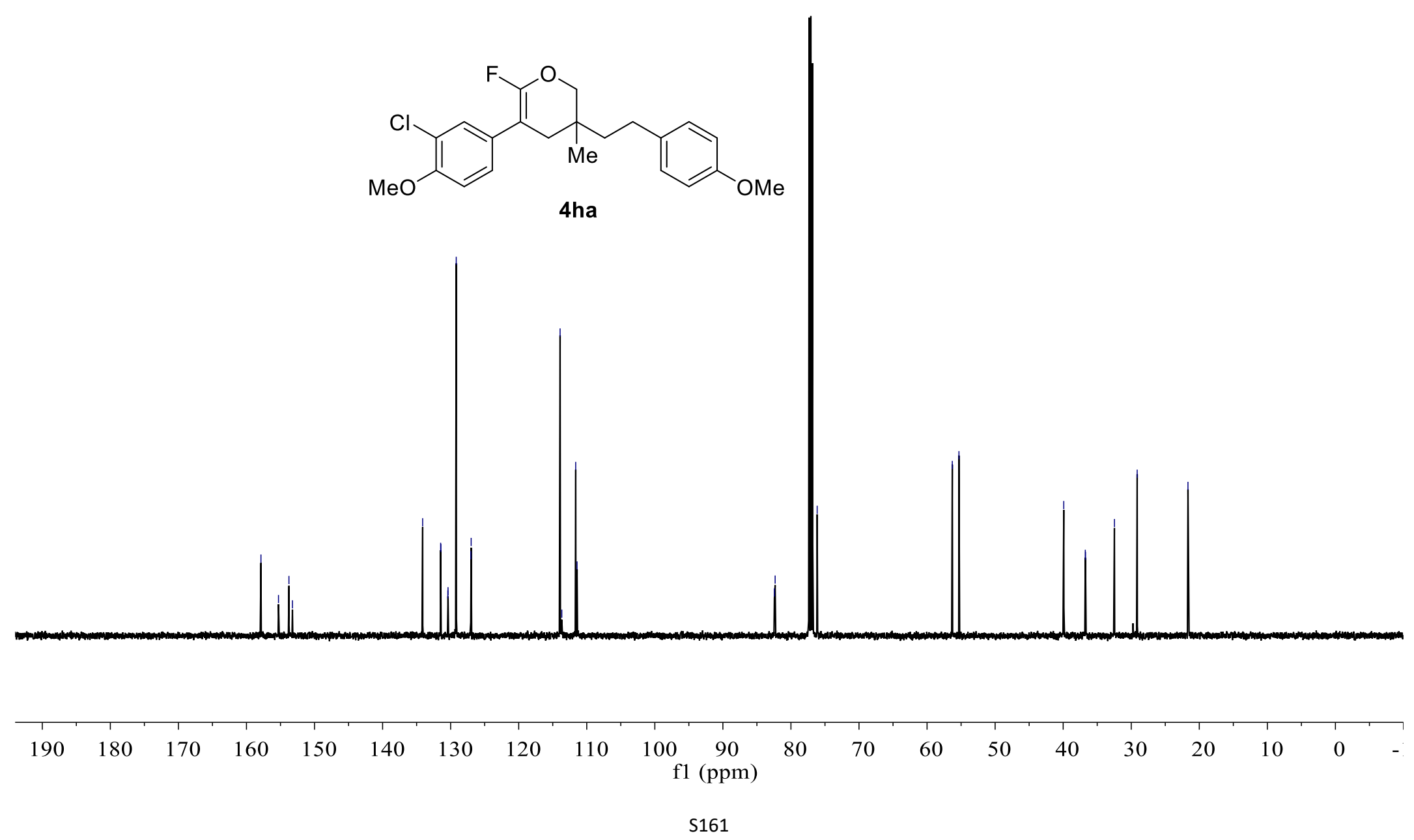




\section{${ }^{19}$ F NMR-spectrum (471 MHz, $\mathrm{CDCl}_{3}$ ) of 4 ha}

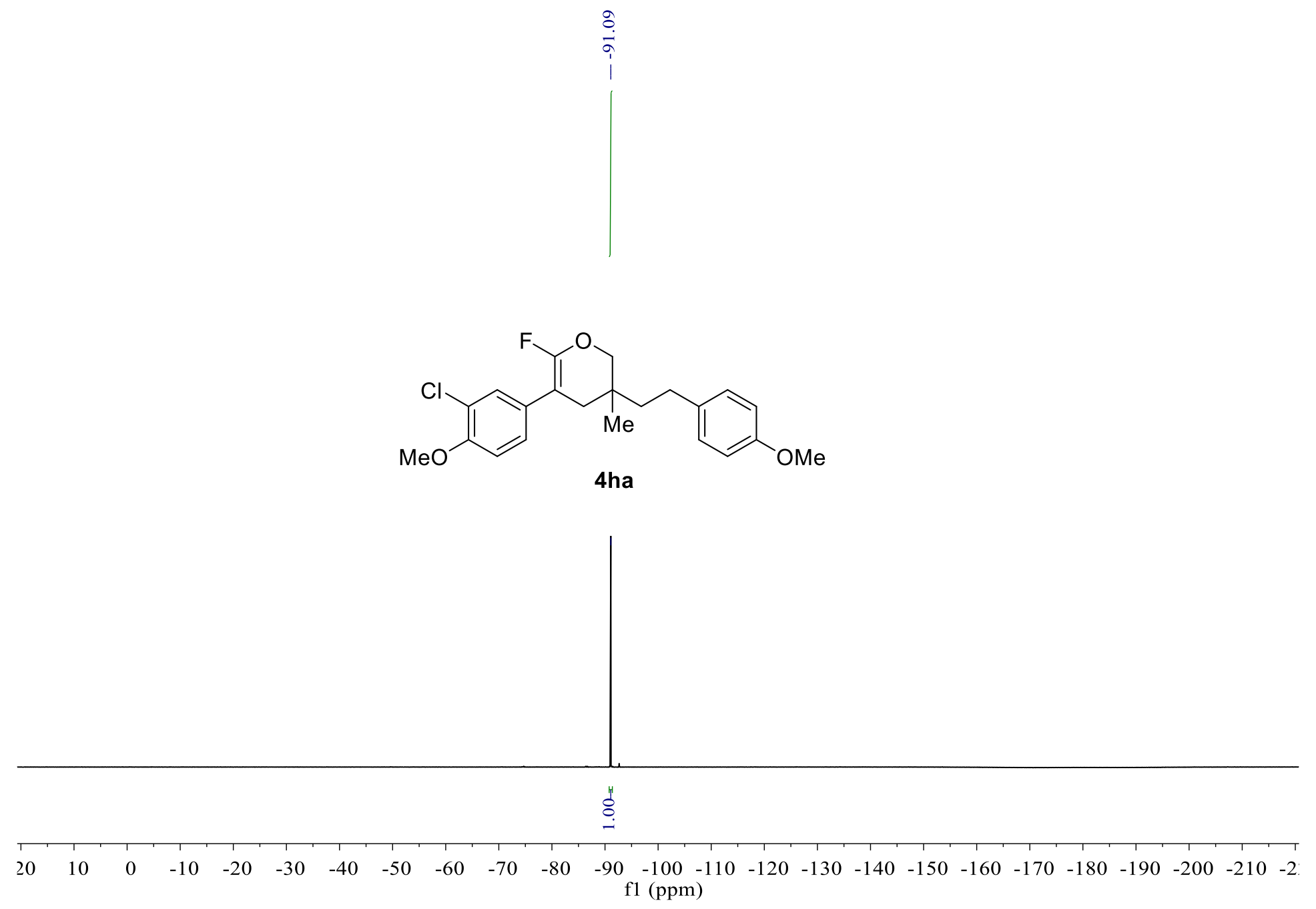



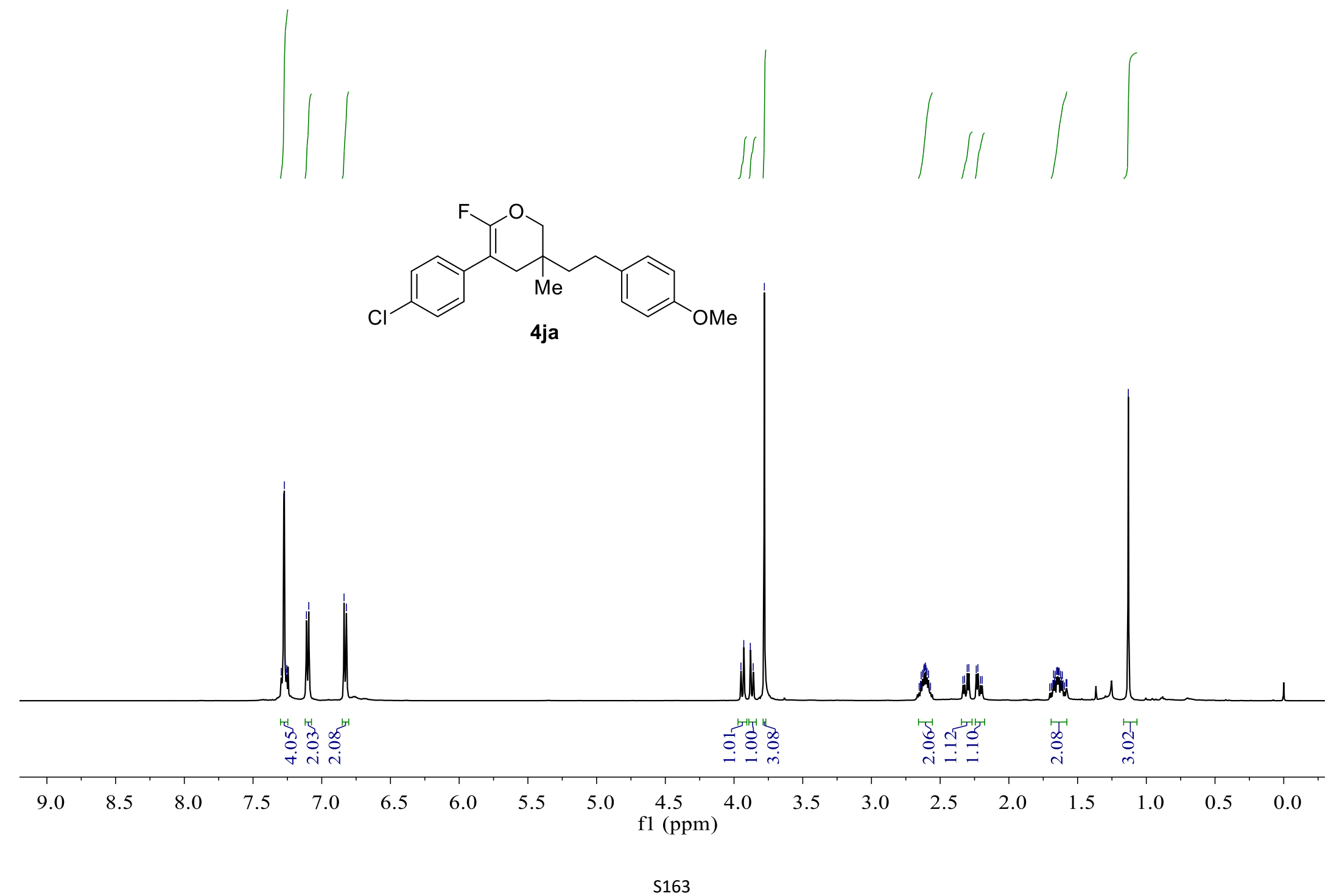
${ }^{13} \mathrm{C}$ NMR-spectrum $\left(126 \mathrm{MHz}, \mathrm{CDCl}_{3}\right)$ of $\mathbf{4 j a}$
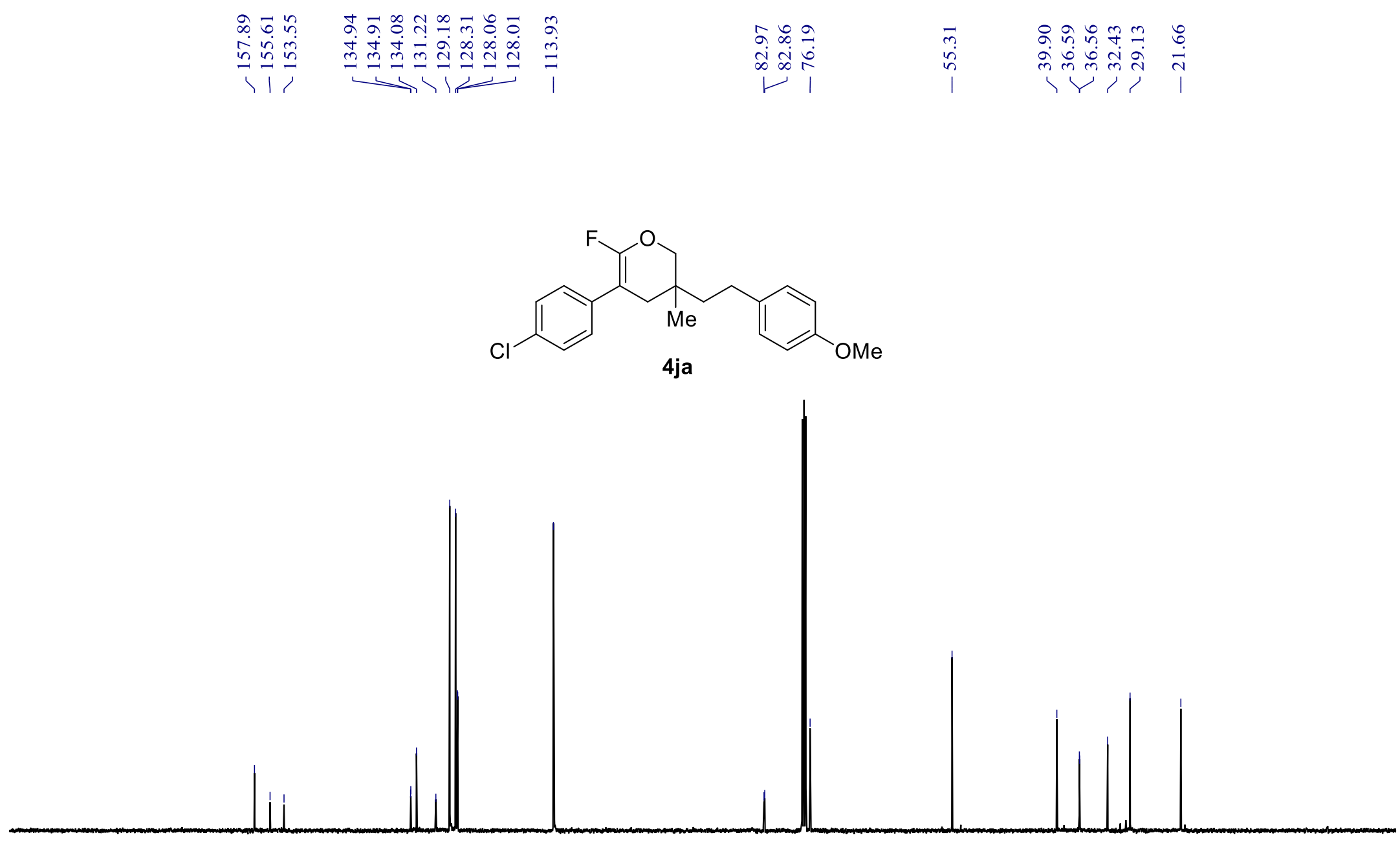

$\begin{array}{lllllll}190 & 180 & 170 & 160 & 150 & 140 & 130\end{array}$

$$
\text { f1 (ppm) }
$$$$
\begin{array}{lllllll}
60 & 50 & 40 & 30 & 20 & 10 & 0
\end{array}
$$ 
${ }^{19} \mathrm{~F}$ NMR-spectrum $\left(471 \mathrm{MHz}, \mathrm{CDCl}_{3}\right)$ of $\mathbf{4 j a}$

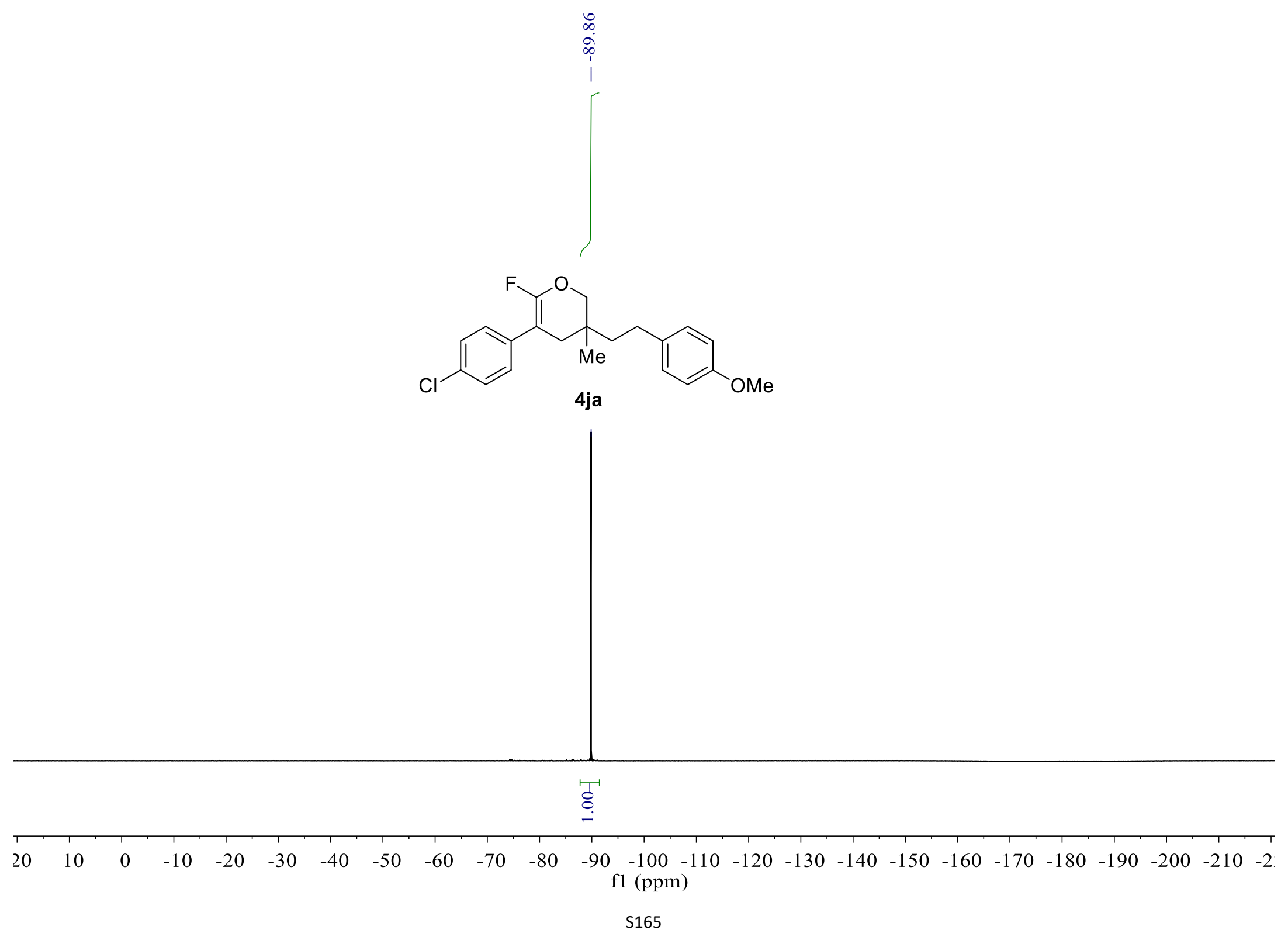




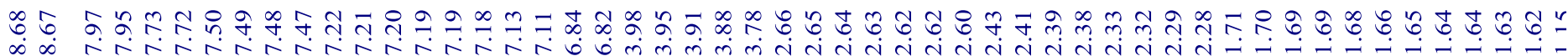
$\checkmark>120$

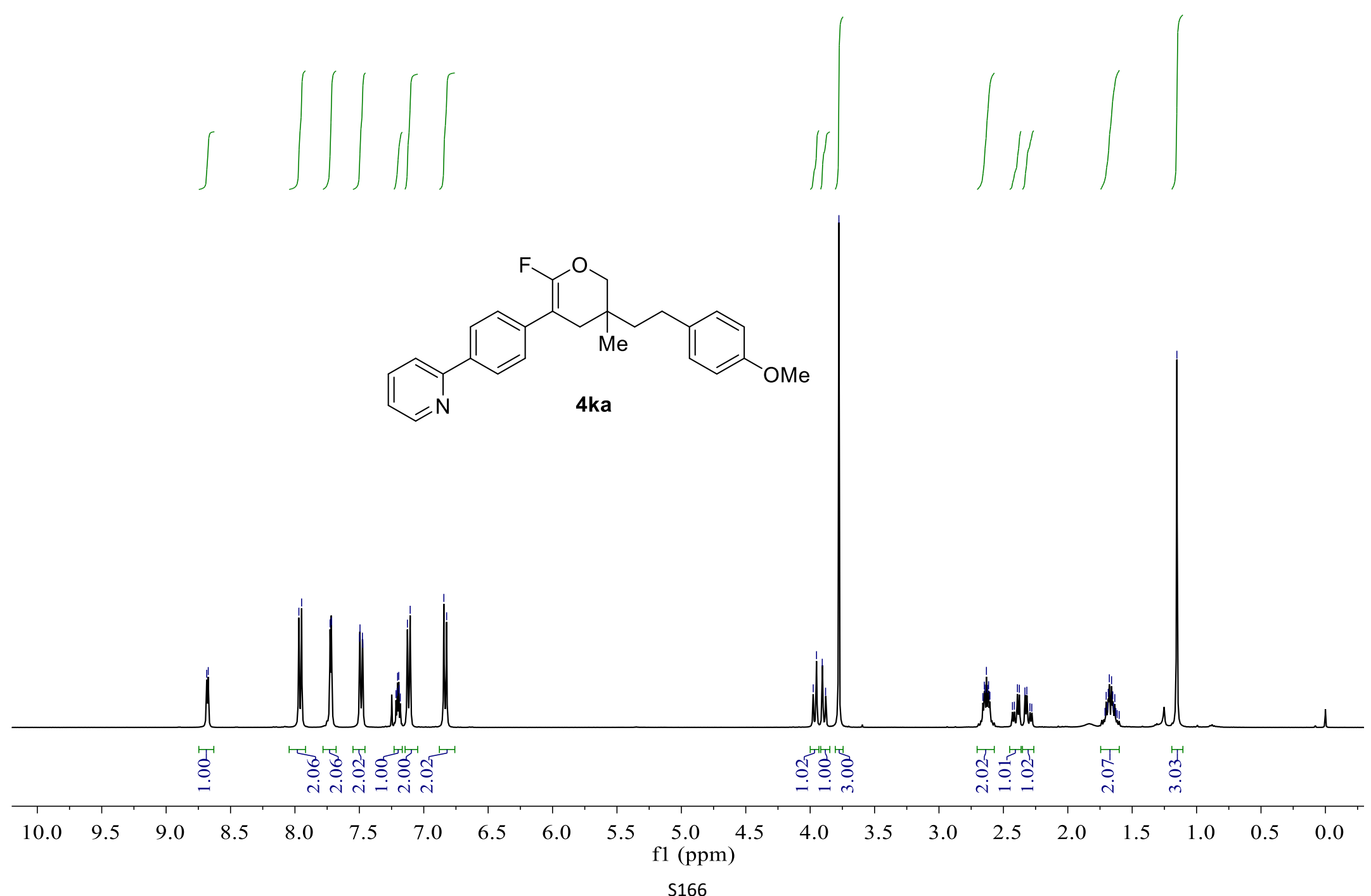


${ }^{13} \mathrm{C} \mathrm{NMR}$-spectrum $\left(101 \mathrm{MHz}, \mathrm{CDCl}_{3}\right)$ of $\mathbf{4} \mathbf{k a}$
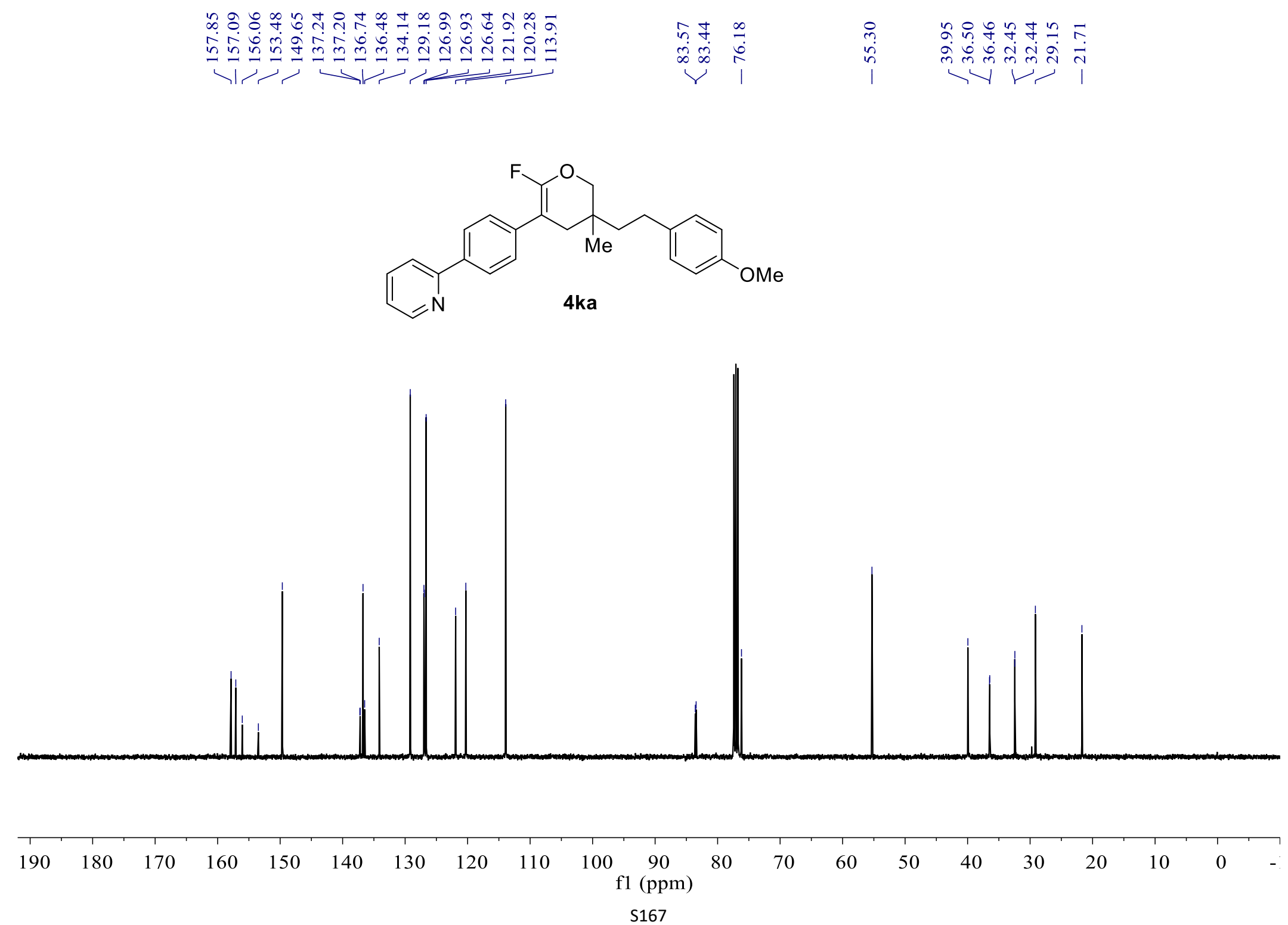
${ }^{19} \mathrm{~F}$ NMR-spectrum $\left(376 \mathrm{MHz}, \mathrm{CDCl}_{3}\right.$ ) of $\mathbf{4 k a}$

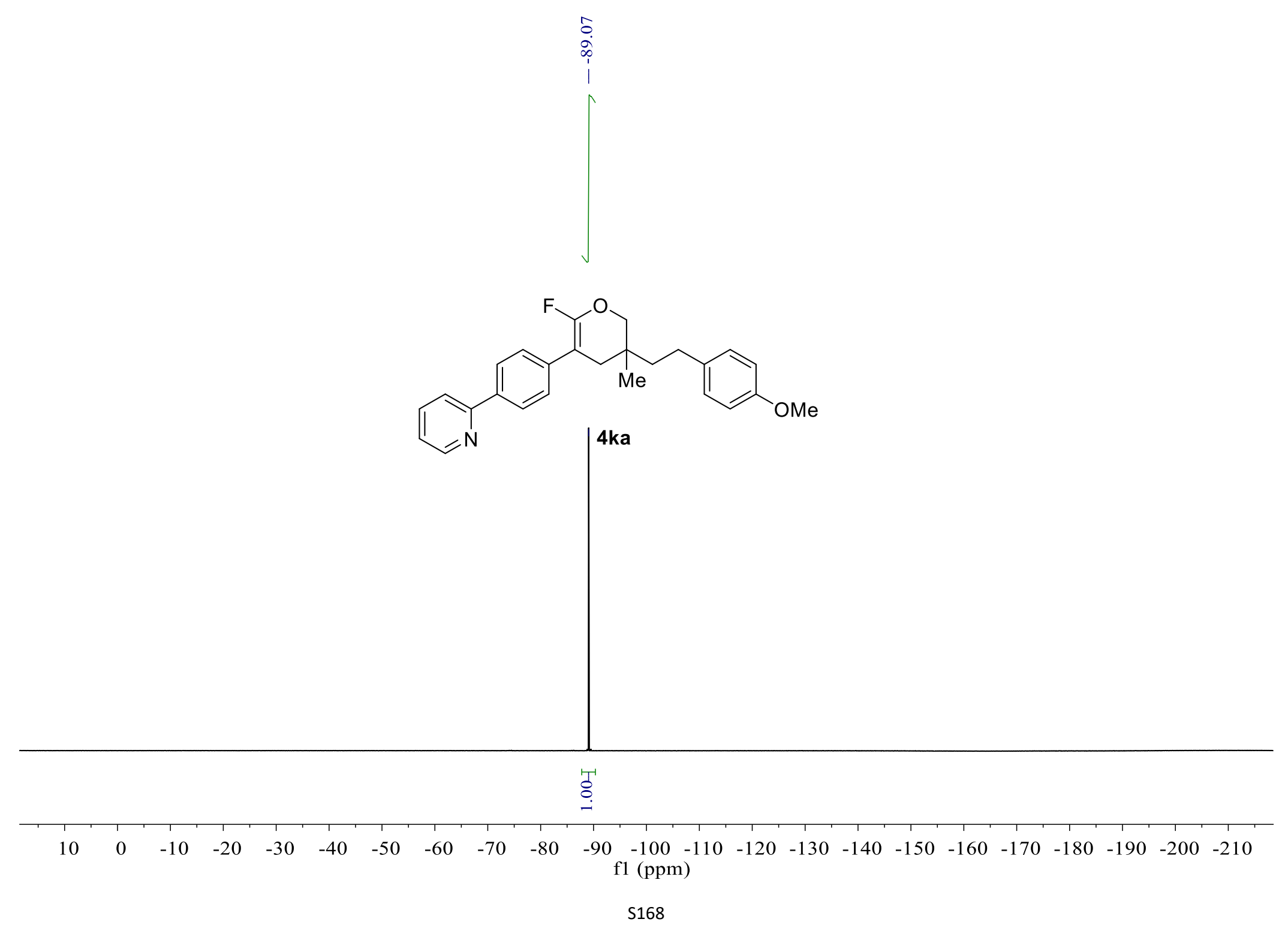


${ }^{1} \mathrm{H}$ NMR-spectrum $\left(500 \mathrm{MHz}, \mathrm{CDCl}_{3}\right.$ ) of $\mathbf{4 n a}$

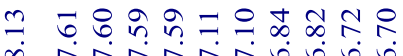

inisine

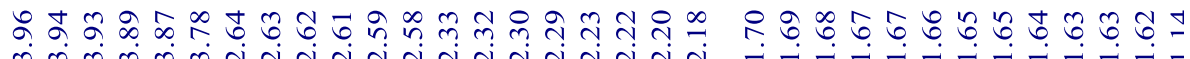
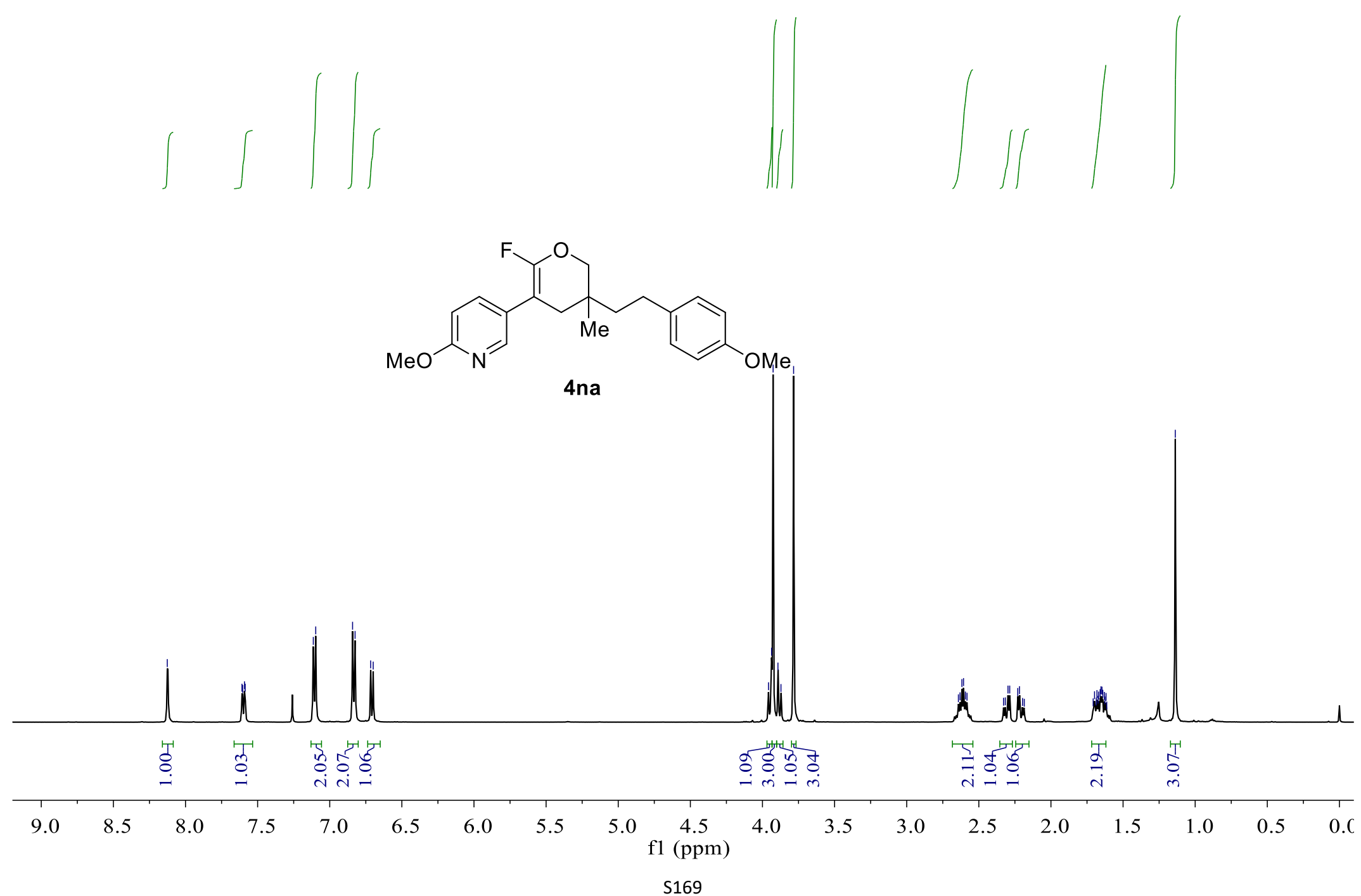
${ }^{13} \mathrm{C}$ NMR-spectrum (126 MHz, $\mathrm{CDCl}_{3}$ ) of 4 na

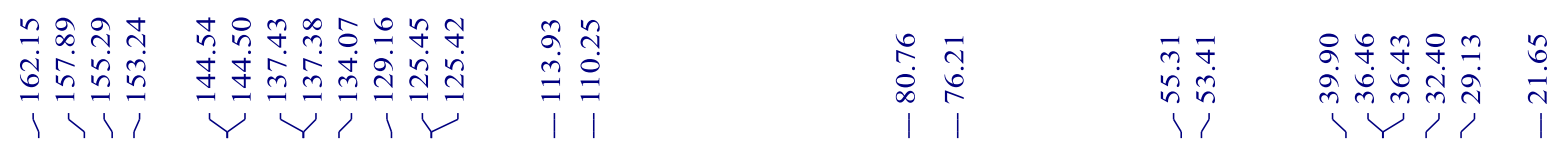

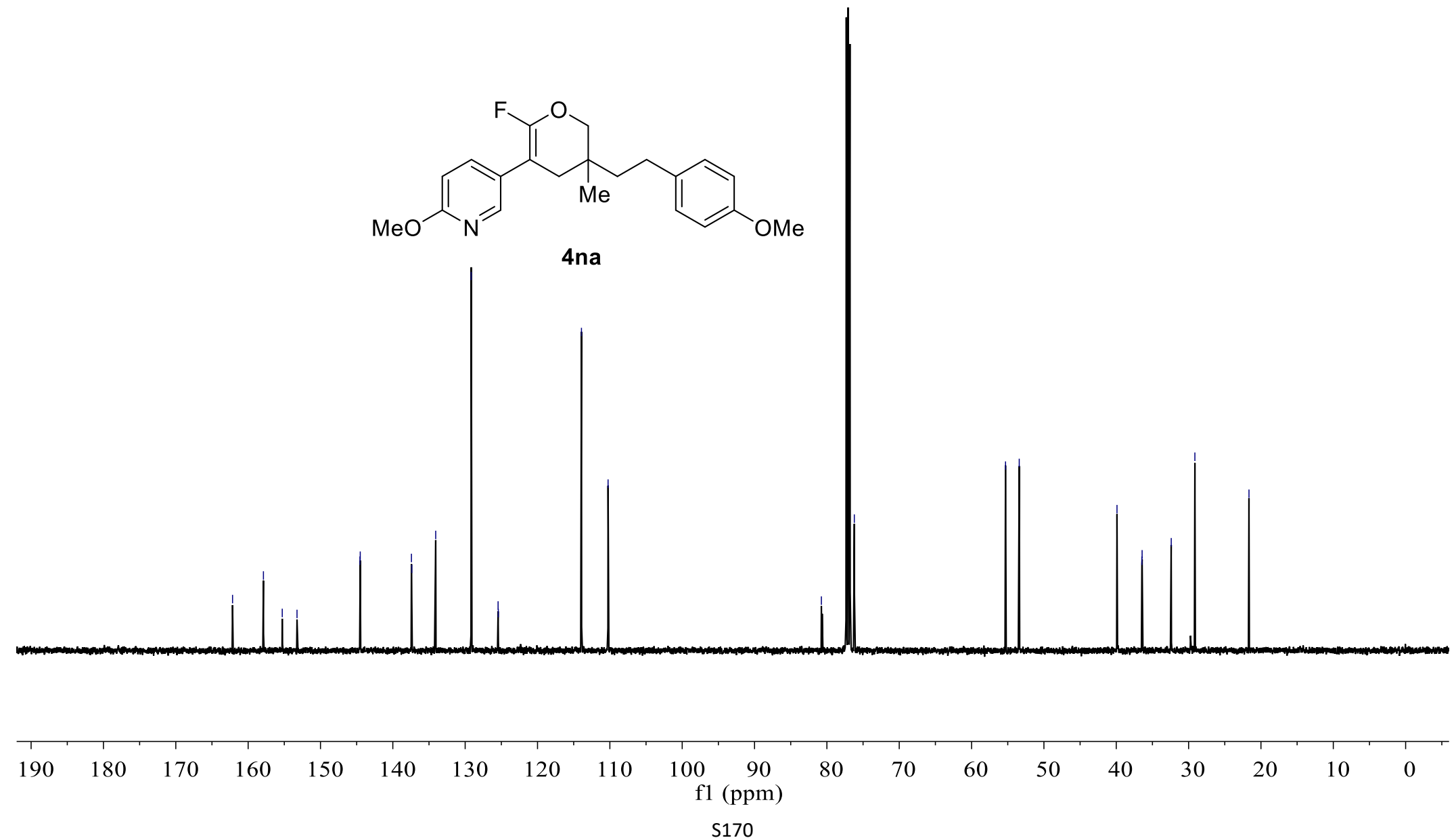


${ }^{19} \mathrm{~F}$ NMR-spectrum $\left(471 \mathrm{MHz}, \mathrm{CDCl}_{3}\right)$ of 4 na

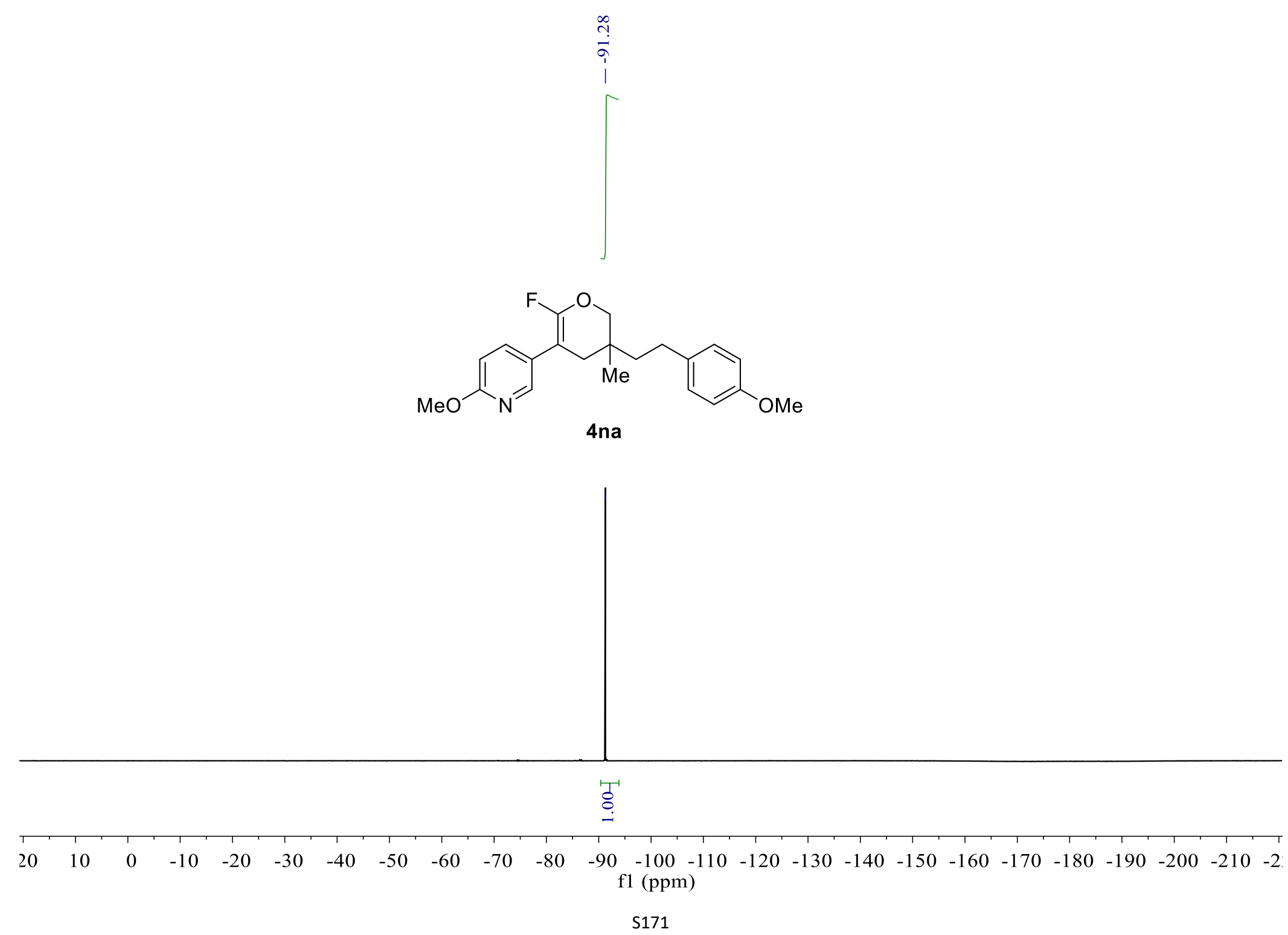




\section{${ }^{1} \mathrm{H}$ NMR-spectrum $\left(400 \mathrm{MHz}, \mathrm{CDCl}_{3}\right)$ of $\mathbf{4 o a}$}

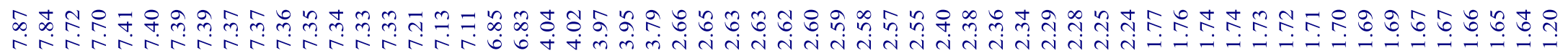
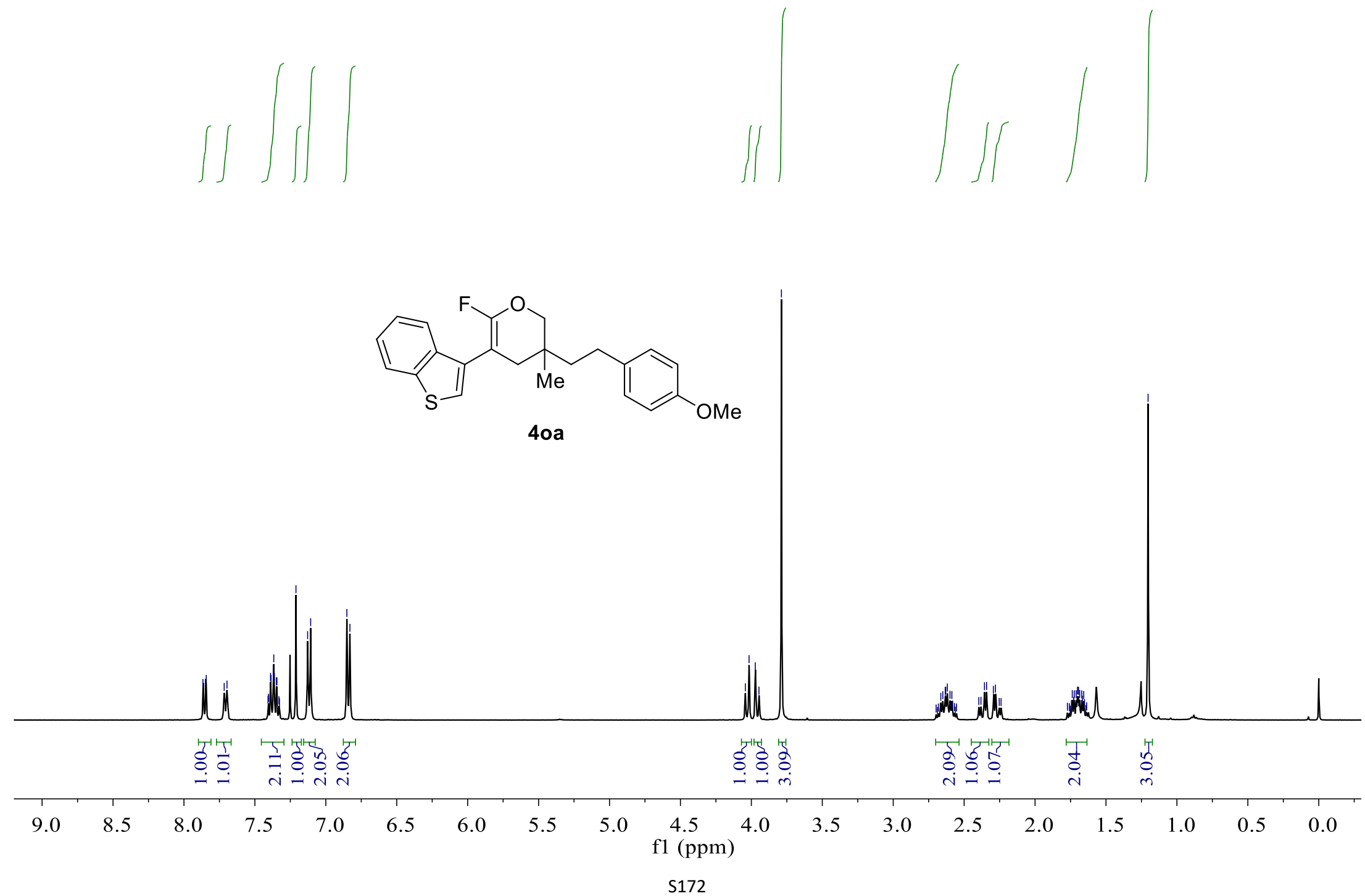
${ }^{13} \mathrm{C}$ NMR-spectrum $\left(101 \mathrm{MHz}, \mathrm{CDCl}_{3}\right)$ of $4 \mathbf{o a}$

$$
\begin{aligned}
& \text { क्্ } \\
& \text { in } \\
& \text { 1) }
\end{aligned}
$$
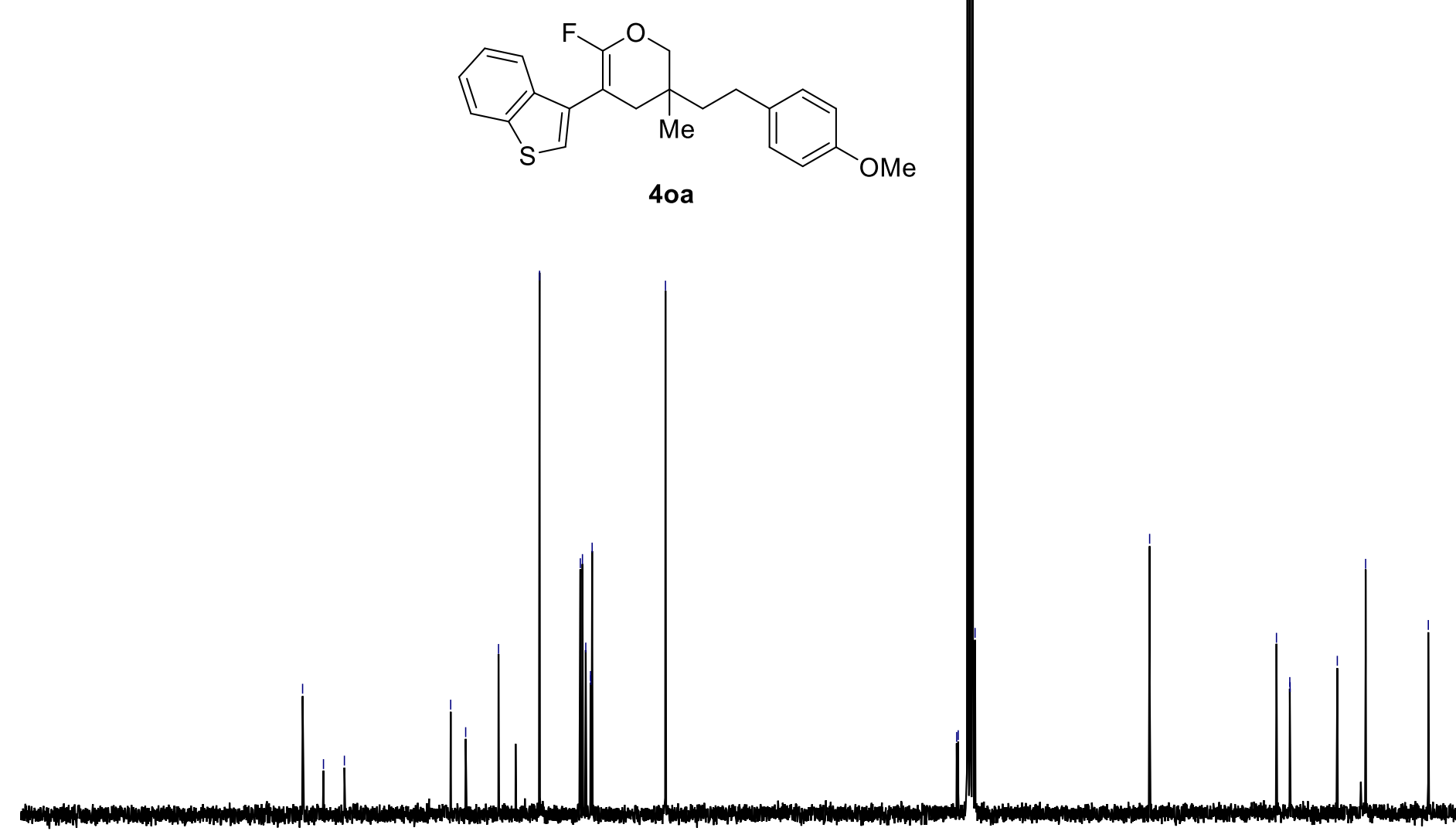
${ }^{19} \mathrm{~F}$ NMR-spectrum $\left(376 \mathrm{MHz}, \mathrm{CDCl}_{3}\right.$ ) of $\mathbf{4 o a}$

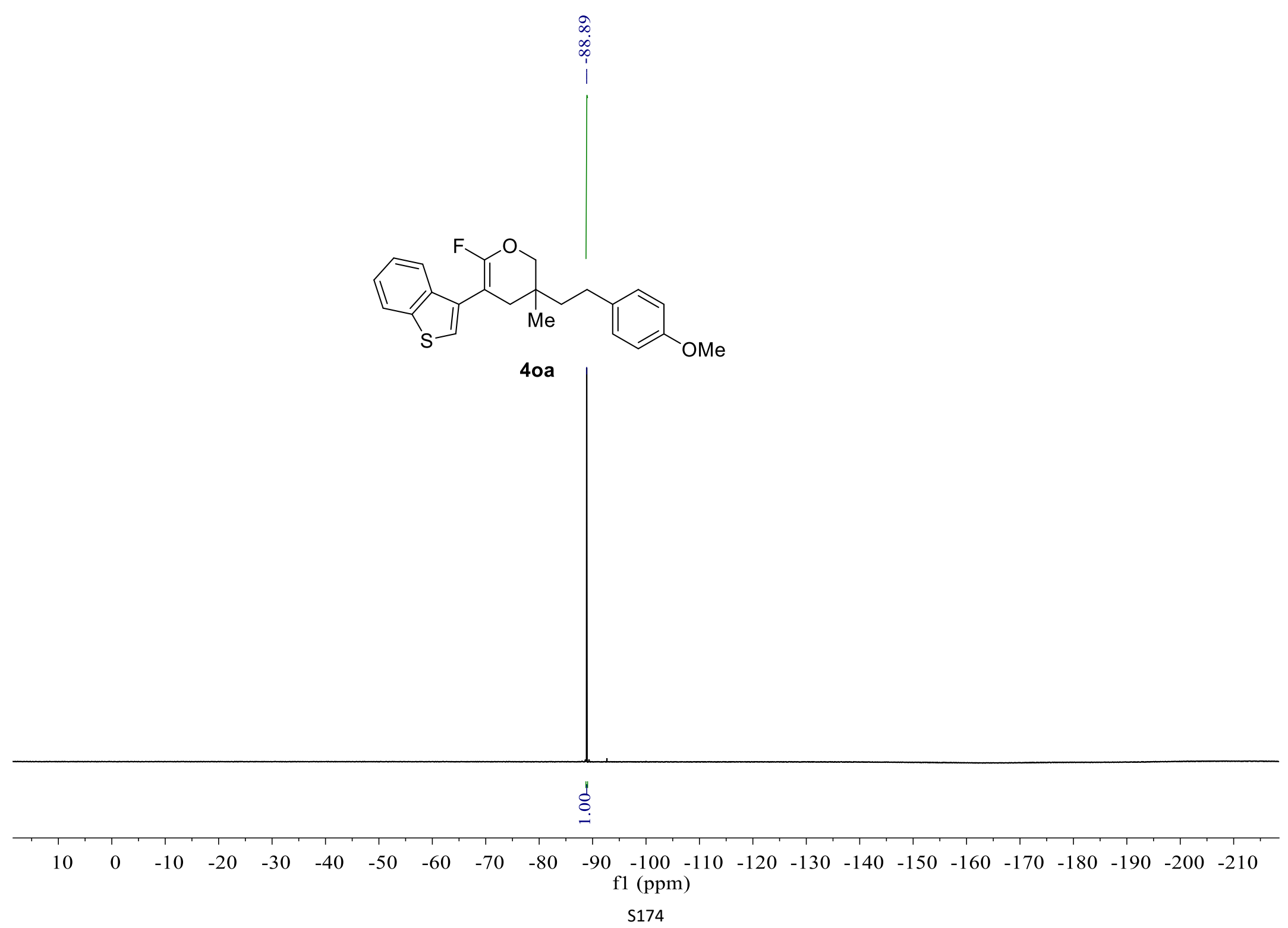


${ }^{1} \mathrm{H}$ NMR-spectrum $\left(500 \mathrm{MHz}, \mathrm{CDCl}_{3}\right.$ ) of $\mathbf{4 a b}$
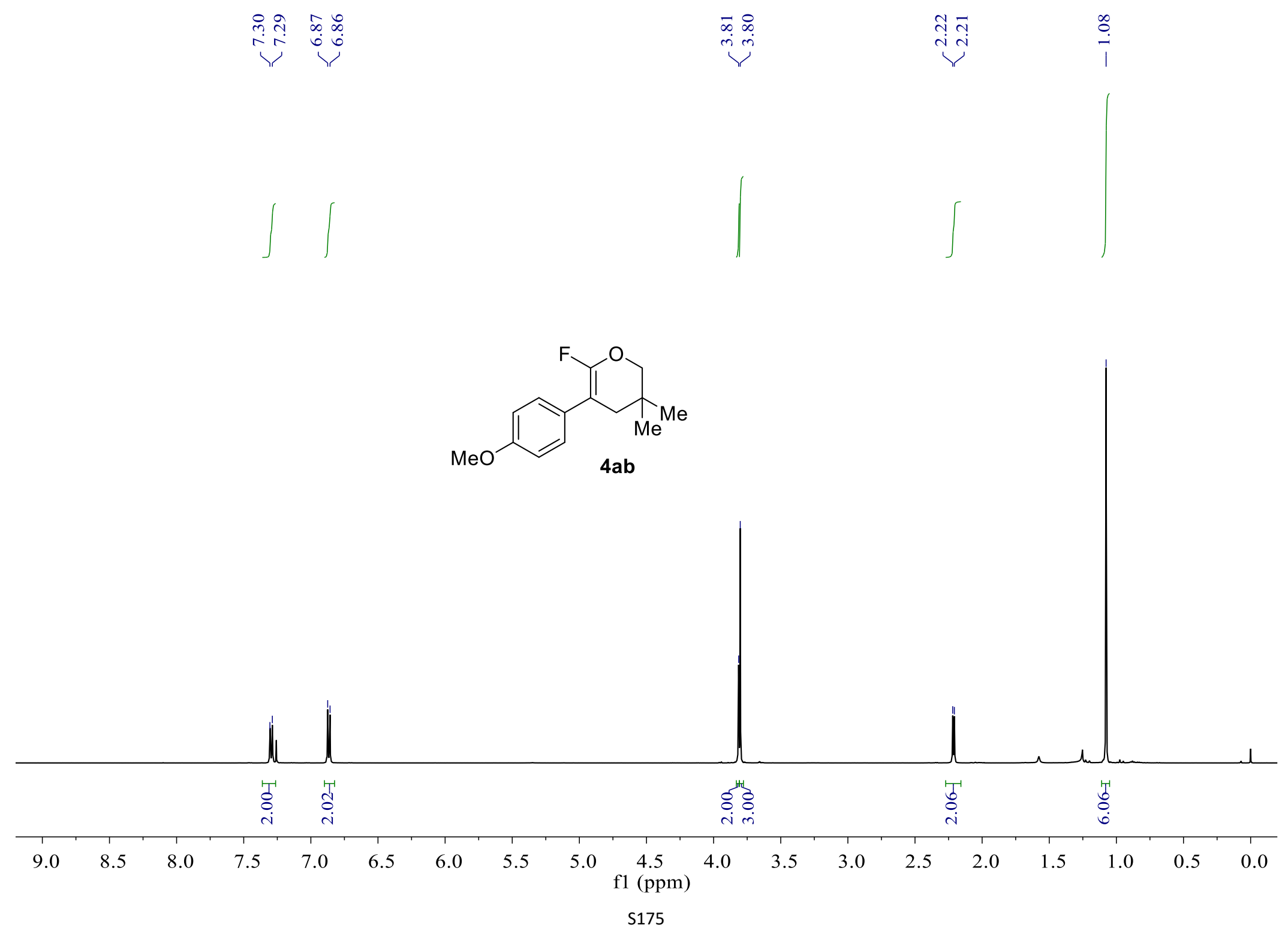
${ }^{13} \mathrm{C}$ NMR-spectrum (126 MHz, $\mathrm{CDCl}_{3}$ ) of $\mathbf{4 a b}$

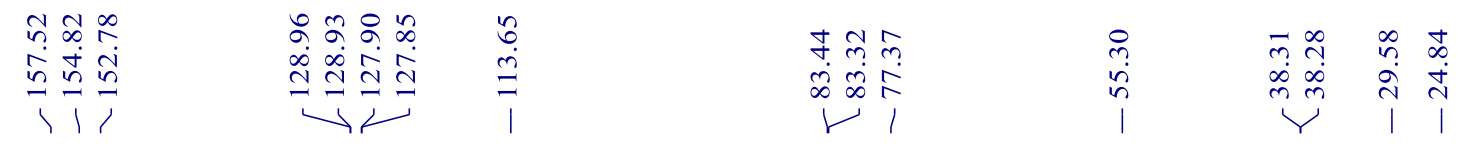

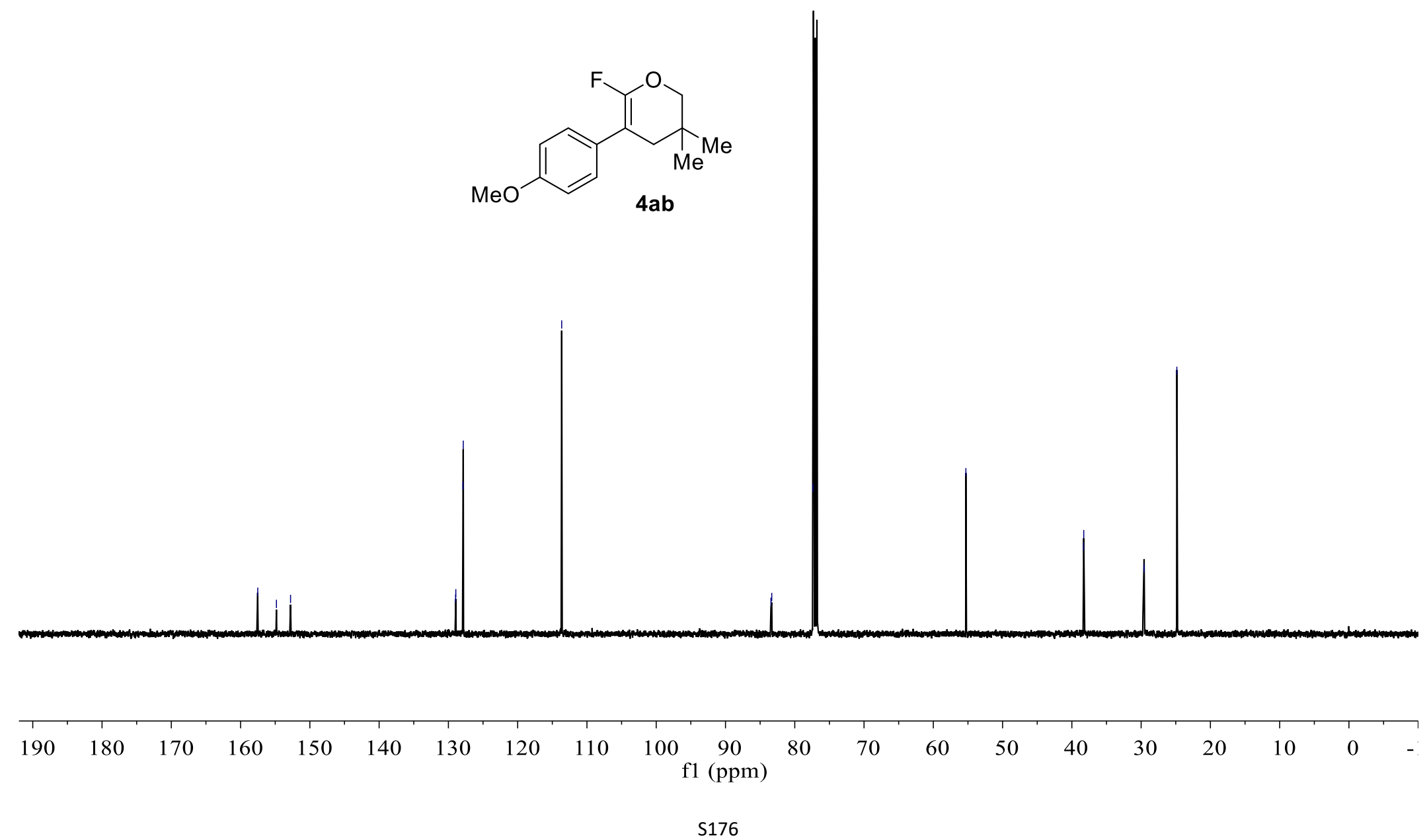


${ }^{19} \mathrm{~F}$ NMR-spectrum $\left(471 \mathrm{MHz}, \mathrm{CDCl}_{3}\right)$ of $\mathbf{4 a b}$
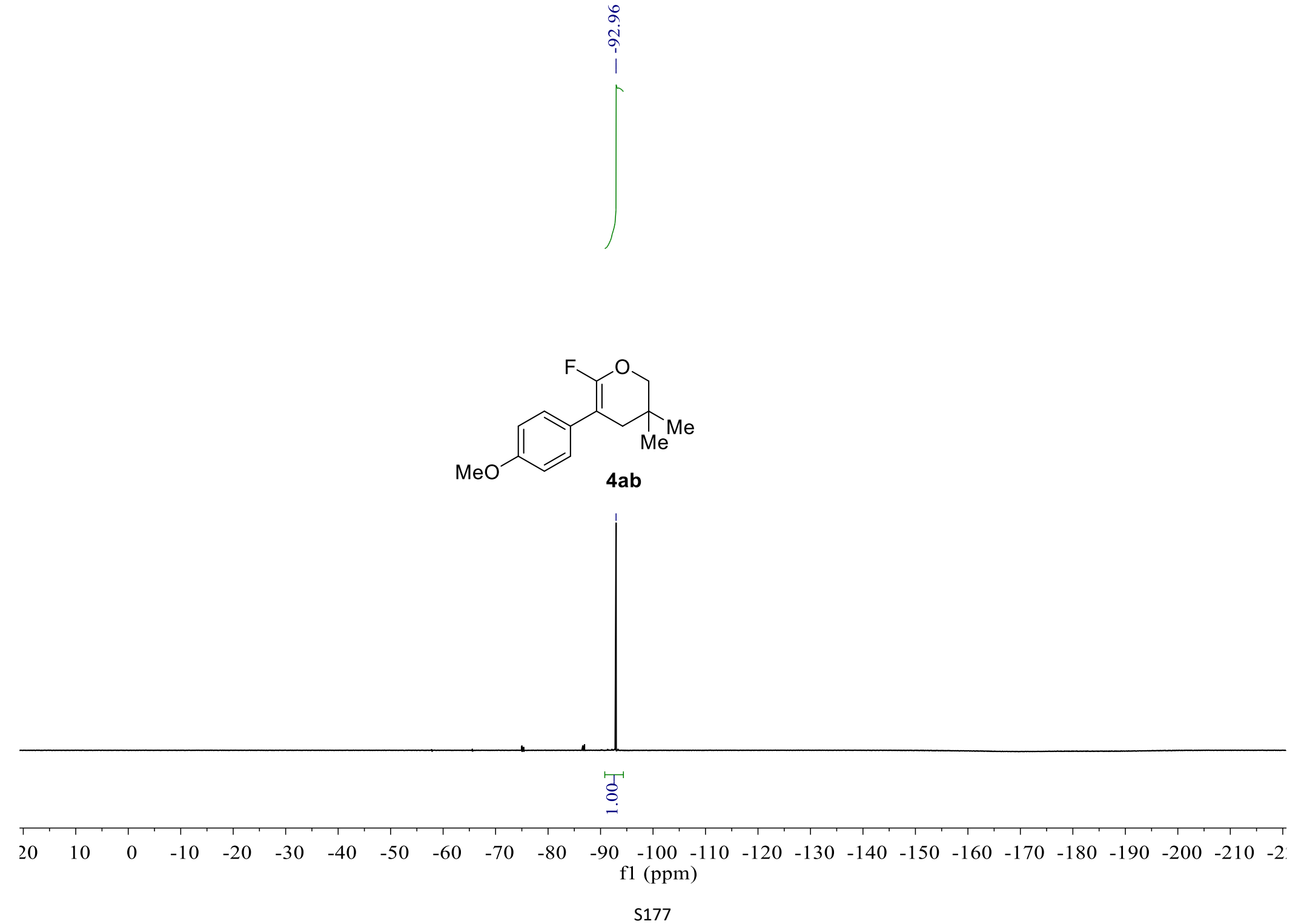
${ }^{1} \mathrm{H}$ NMR-spectrum (400 MHz, $\mathrm{CDCl}_{3}$ ) of $\mathbf{4 a e}$

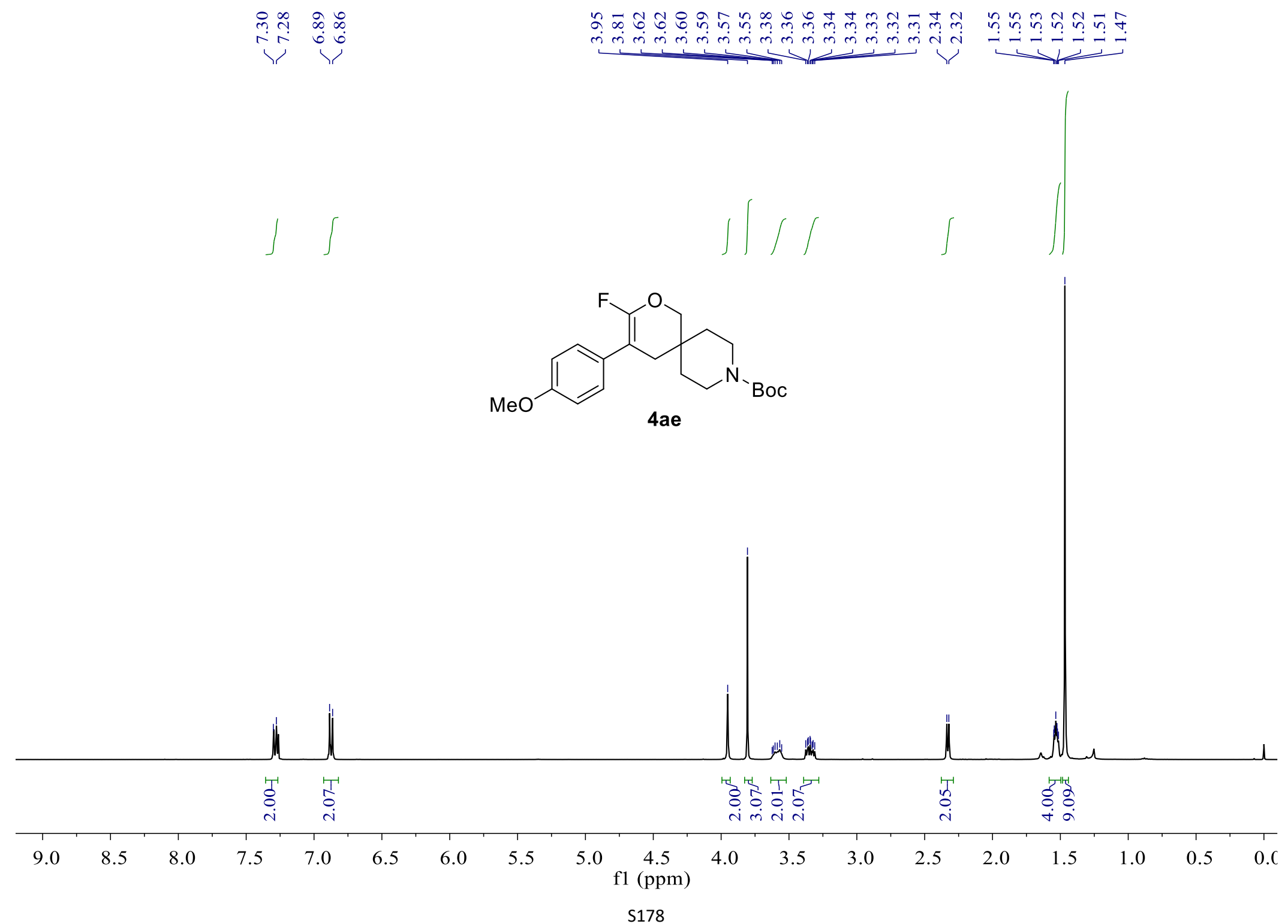


${ }^{13} \mathrm{C}$ NMR-spectrum (101 MHz, $\mathrm{CDCl}_{3}$ ) of $\mathbf{4 a e}$
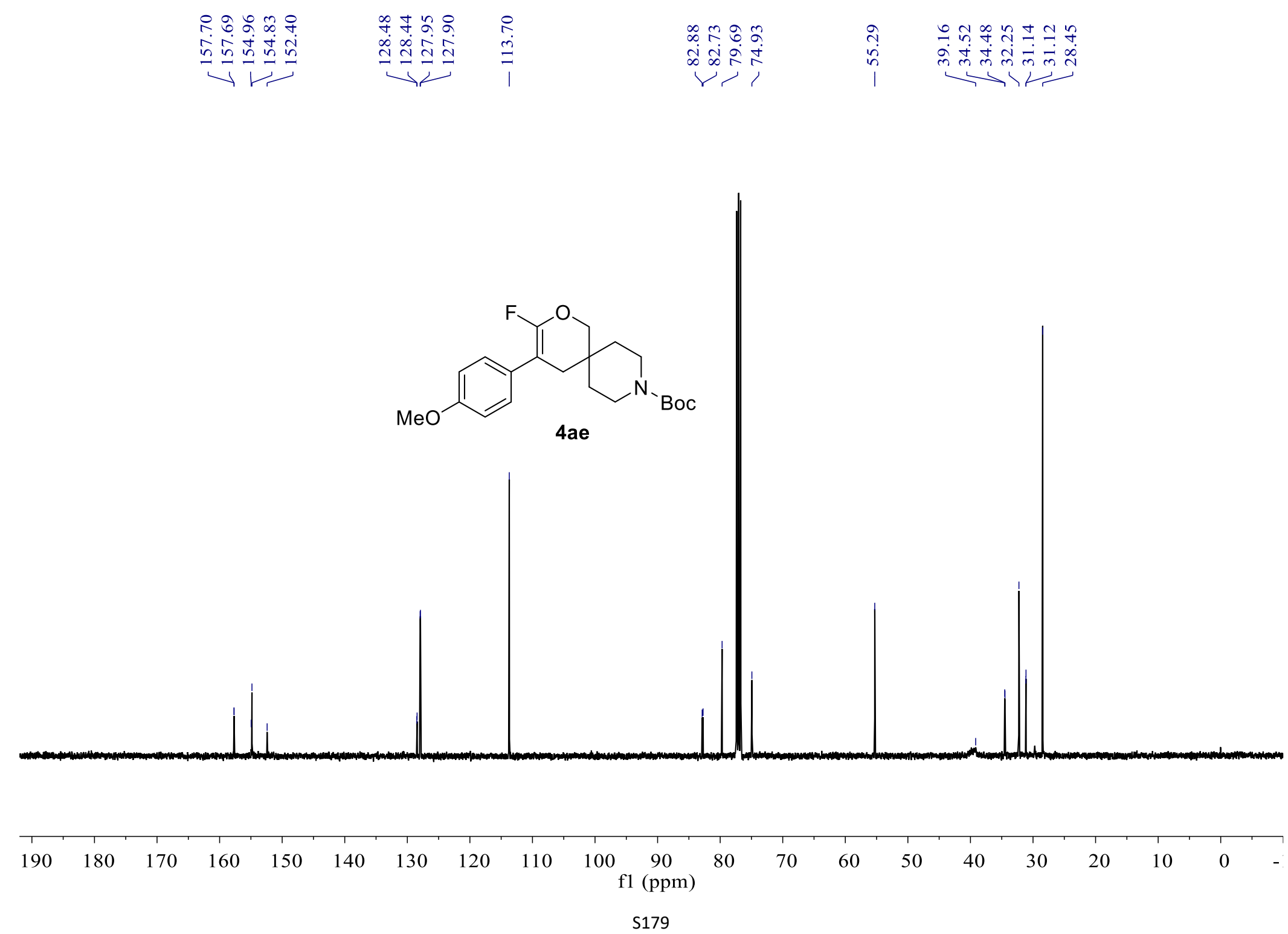
${ }^{19} \mathrm{~F} \mathrm{NMR}$-spectrum $\left(376 \mathrm{MHz}, \mathrm{CDCl}_{3}\right.$ ) of $\mathbf{4 a e}$

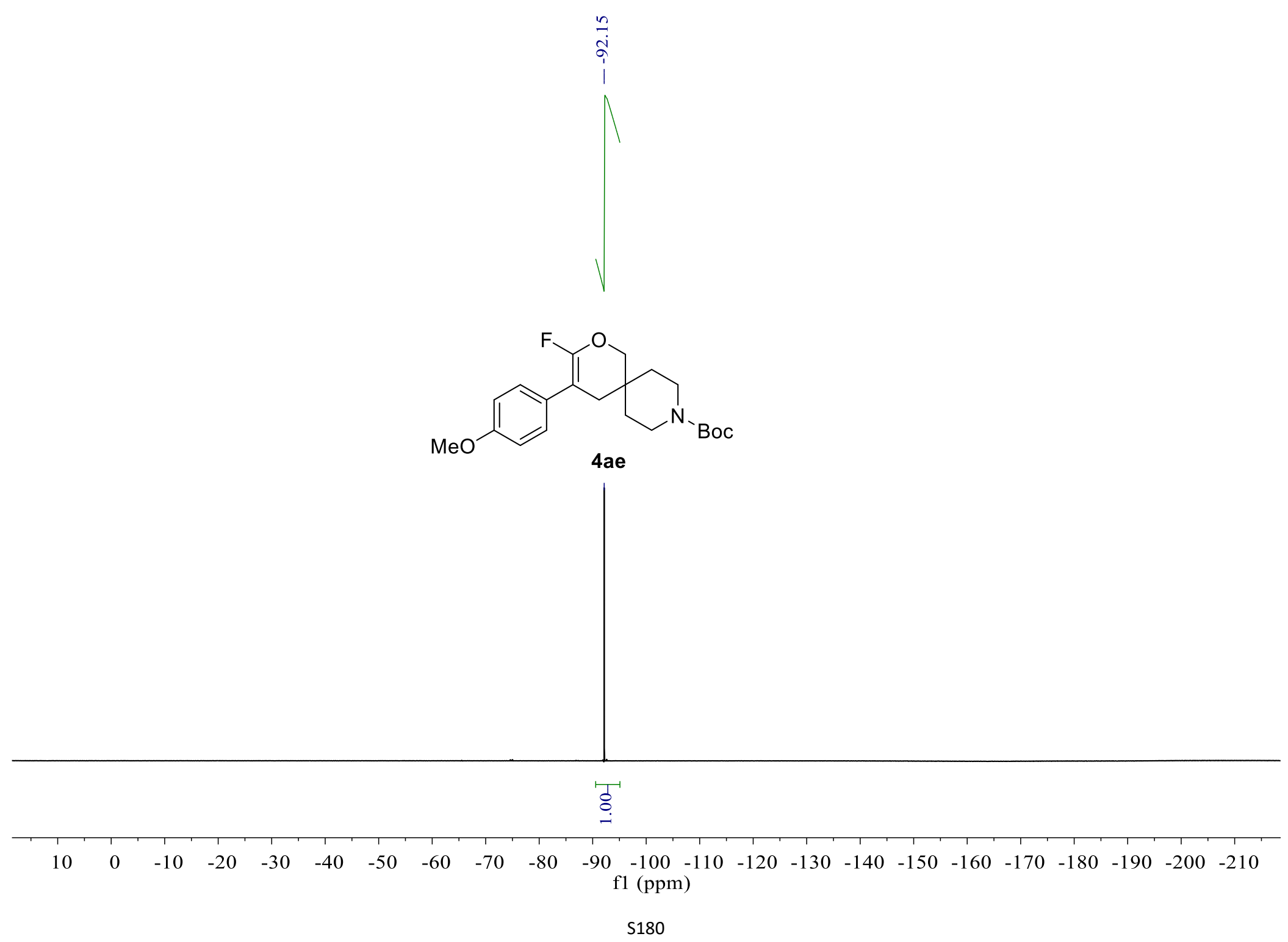


${ }^{1} \mathrm{H}$ NMR-spectrum $\left(500 \mathrm{MHz}, \mathrm{CDCl}_{3}\right.$ ) of $\mathbf{4 a i}$

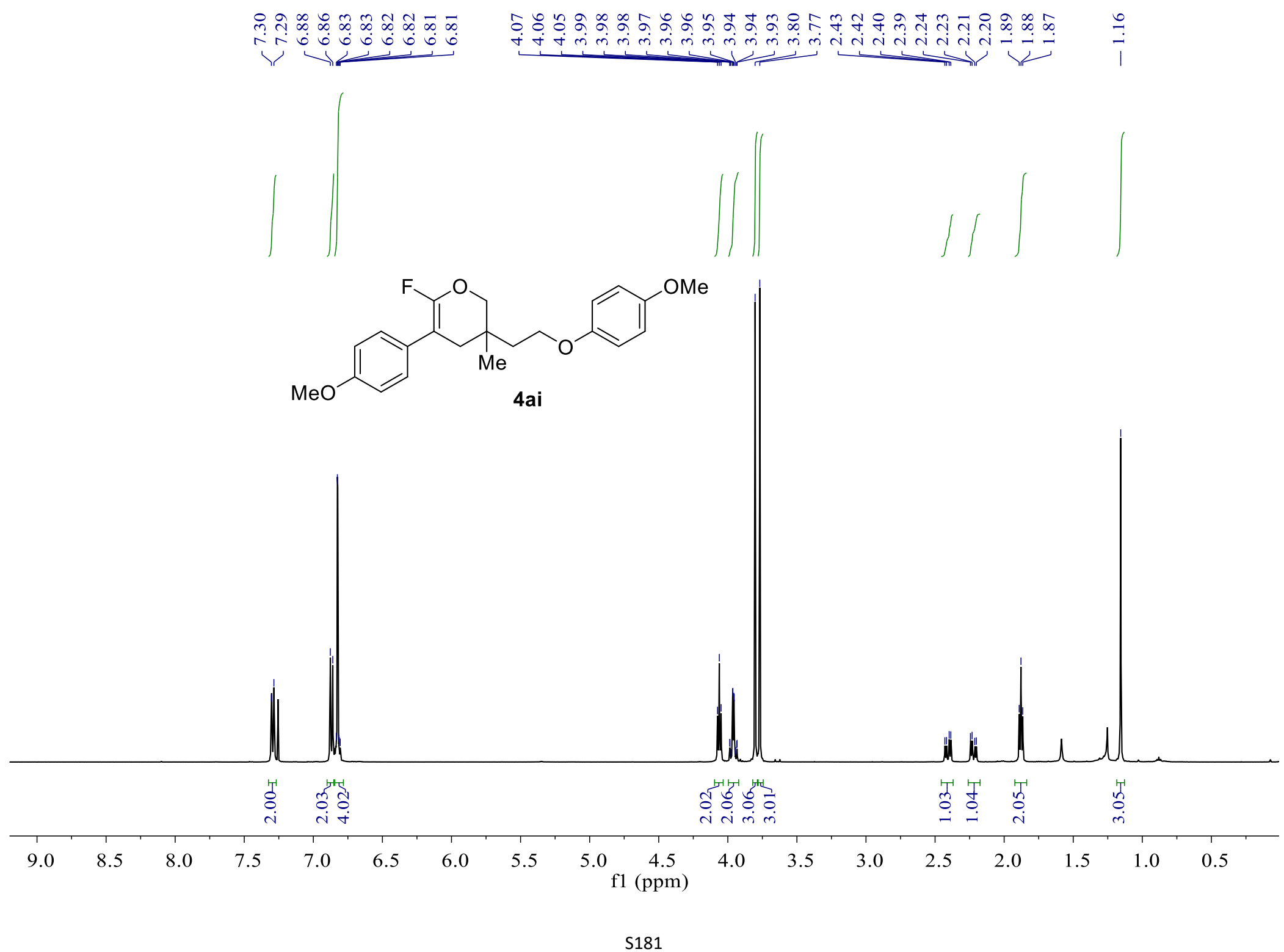


${ }^{13} \mathrm{C} \mathrm{NMR}$-spectrum $\left(126 \mathrm{MHz}, \mathrm{CDCl}_{3}\right)$ of $\mathbf{4 a i}$

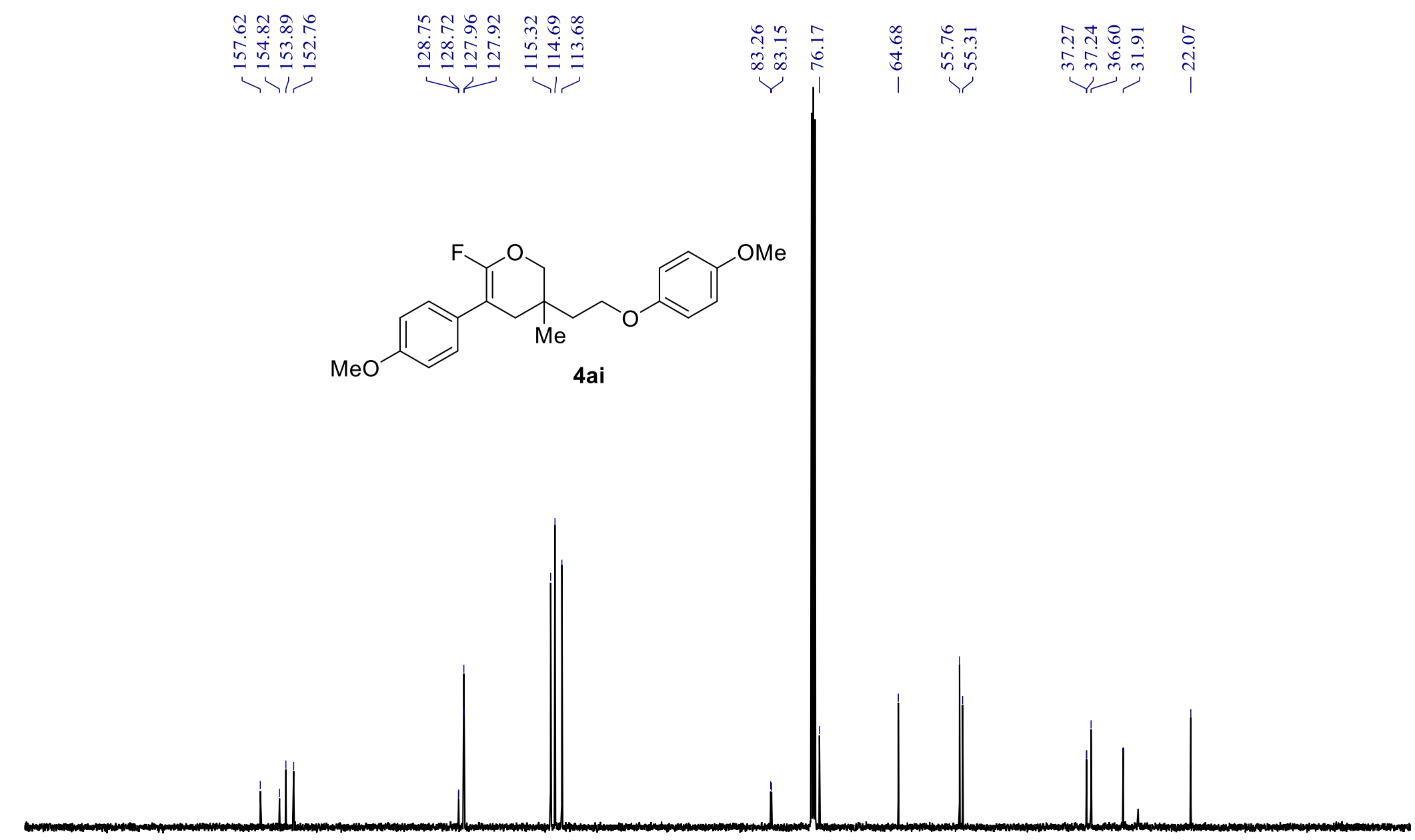

$190 \quad 180$

$170 \quad 160$

150

$140 \quad 13$

120

110100

$100 \underset{\mathrm{f} 1(\mathrm{ppm})}{90} 80$

$70 \quad 60$

50

$40 \quad 30$

$20 \quad 10$

S182 
${ }^{19} \mathrm{~F}$ NMR-spectrum $\left(471 \mathrm{MHz}, \mathrm{CDCl}_{3}\right)$ of $4 \mathbf{a i}$

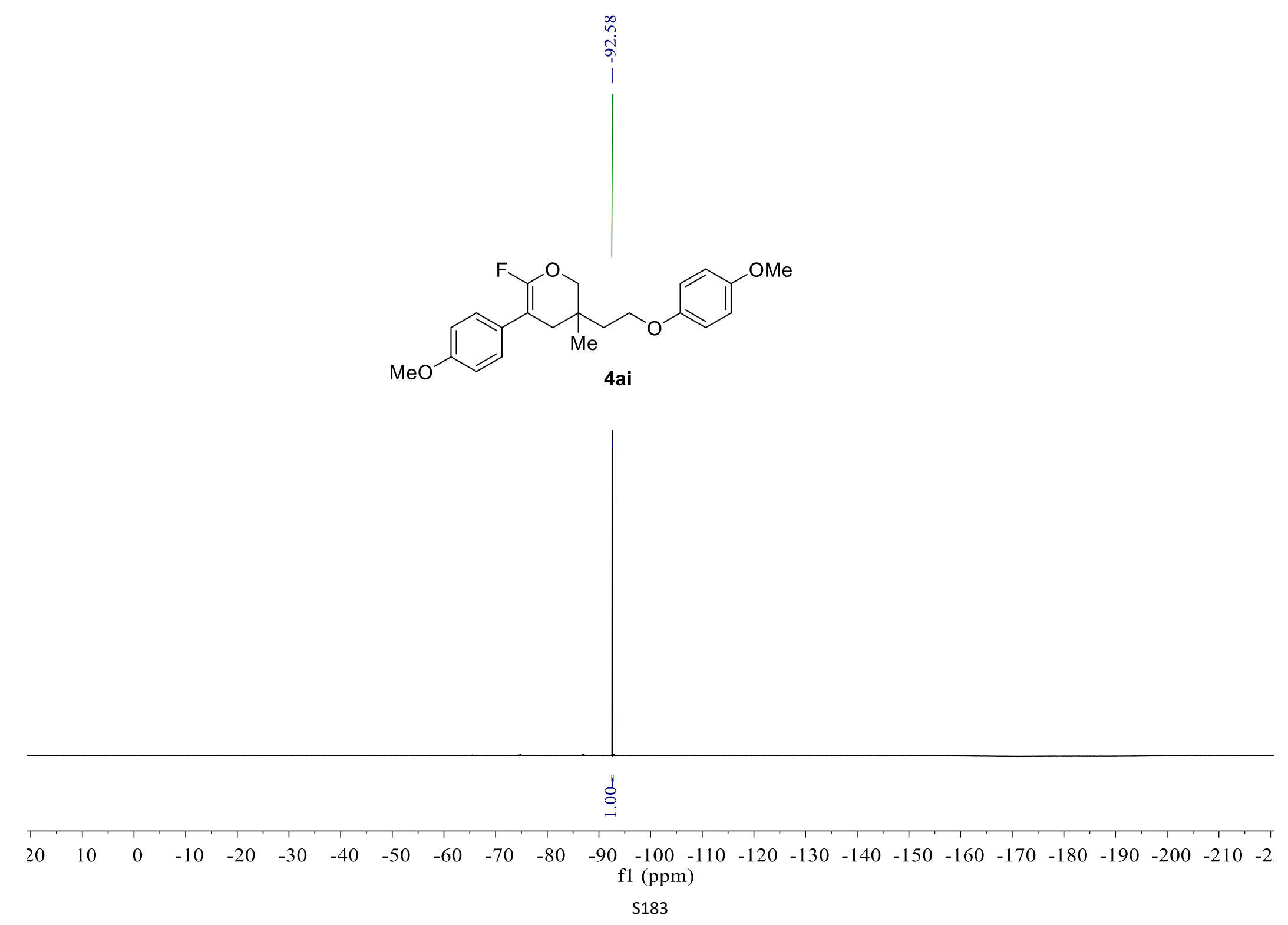


${ }^{1} \mathrm{H}$ NMR-spectrum (400 MHz, $\mathrm{CDCl}_{3}$ ) of $\mathbf{4 a k}$

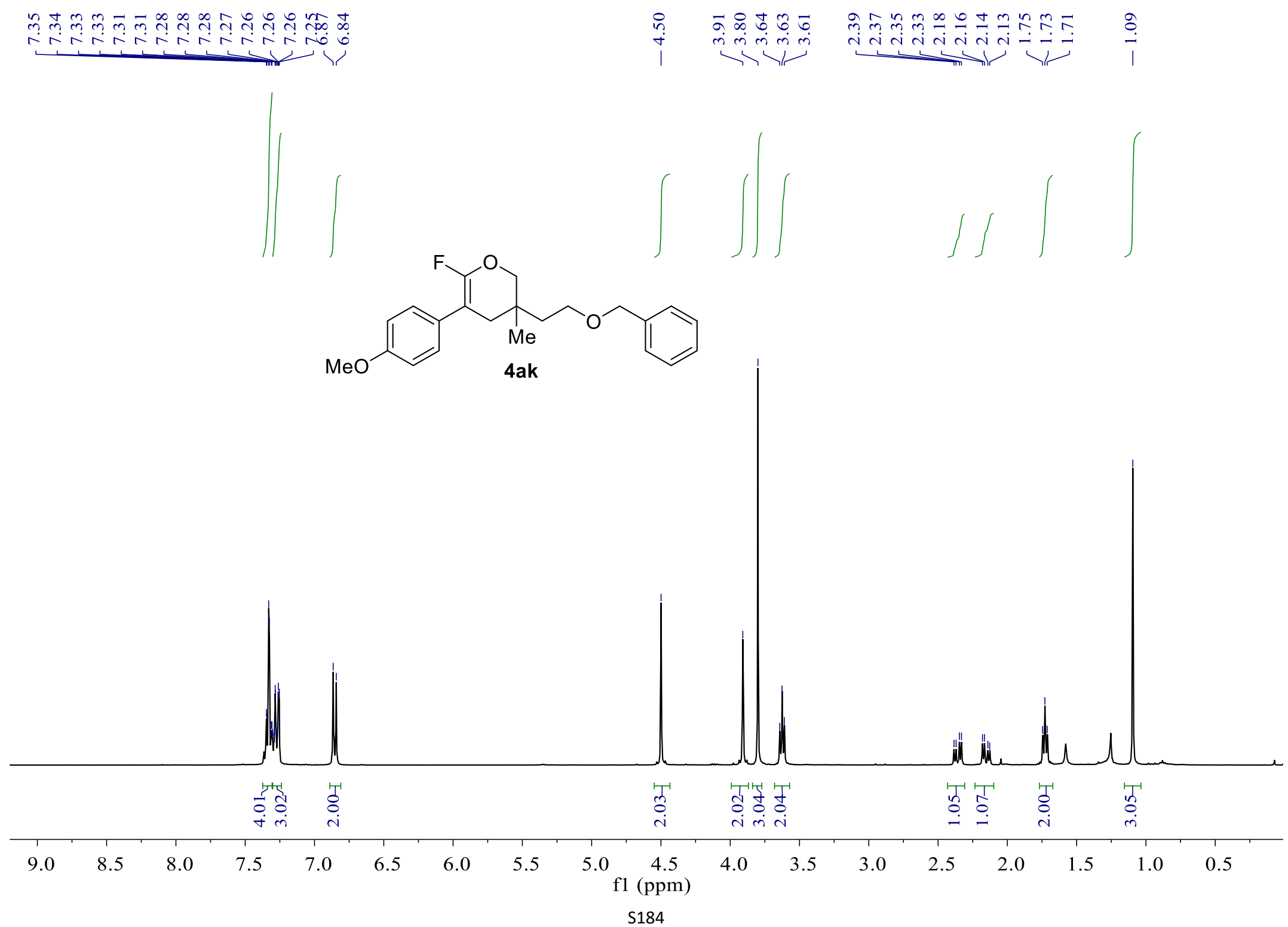


${ }^{13} \mathrm{C}$ NMR-spectrum (101 MHz, $\left.\mathrm{CDCl}_{3}\right)$ of $\mathbf{4 a k}$

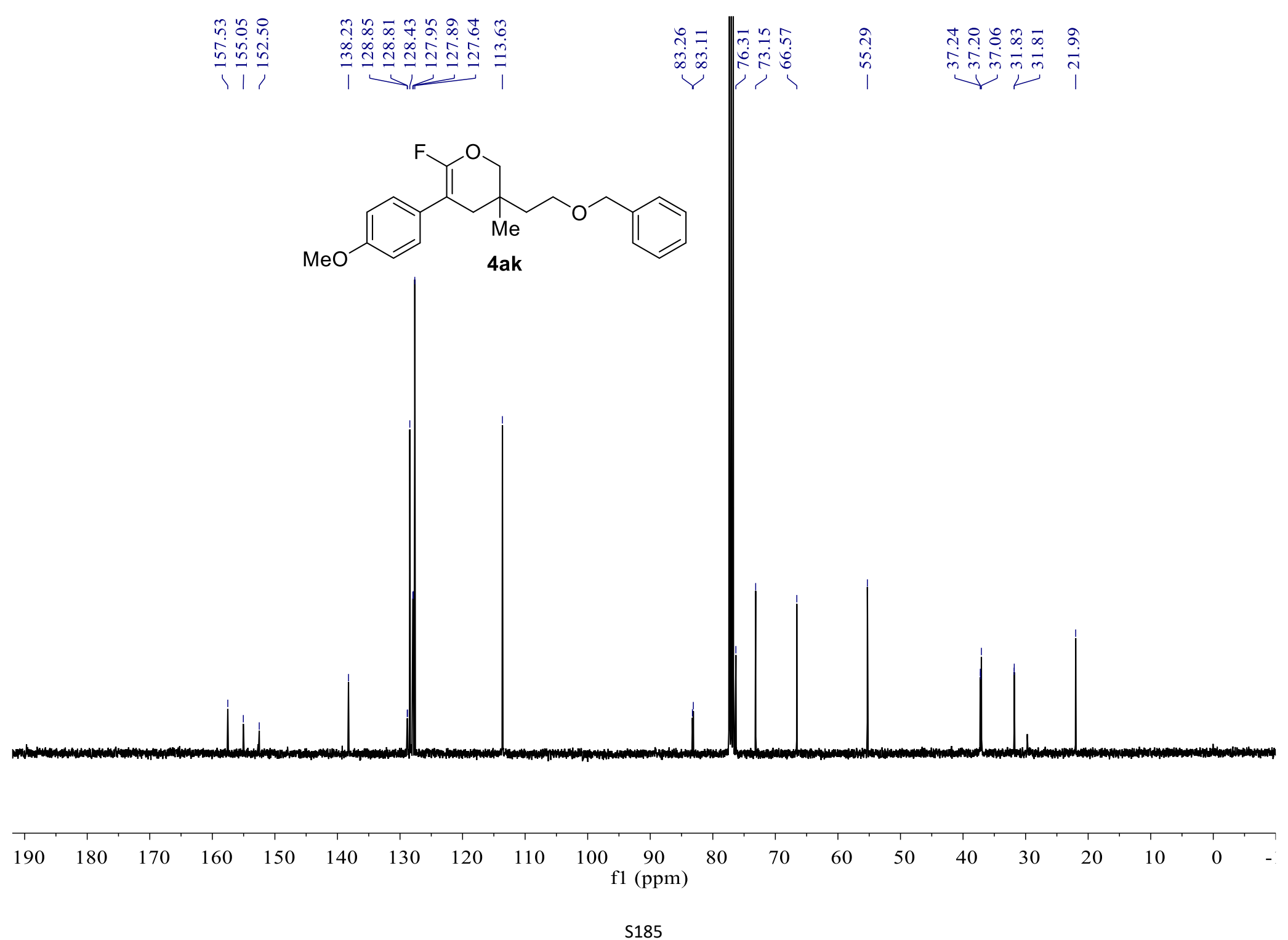


${ }^{19} \mathrm{~F}$ NMR-spectrum $\left(376 \mathrm{MHz}, \mathrm{CDCl}_{3}\right)$ of $\mathbf{4 a k}$

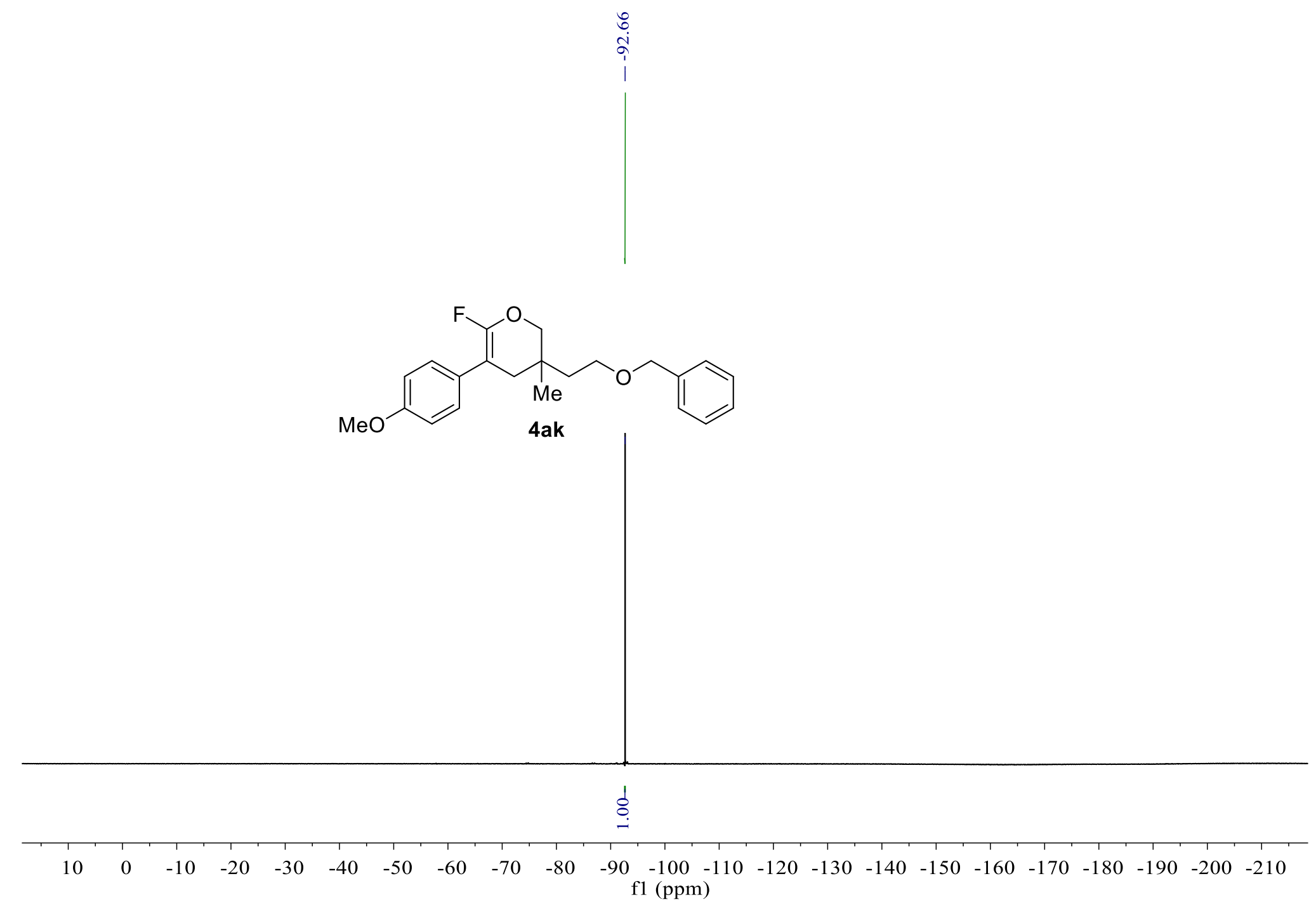

${ }^{1} \mathrm{H}$ NMR-spectrum $\left(500 \mathrm{MHz}, \mathrm{CDCl}_{3}\right.$ ) of $\mathbf{4 a p}$ 

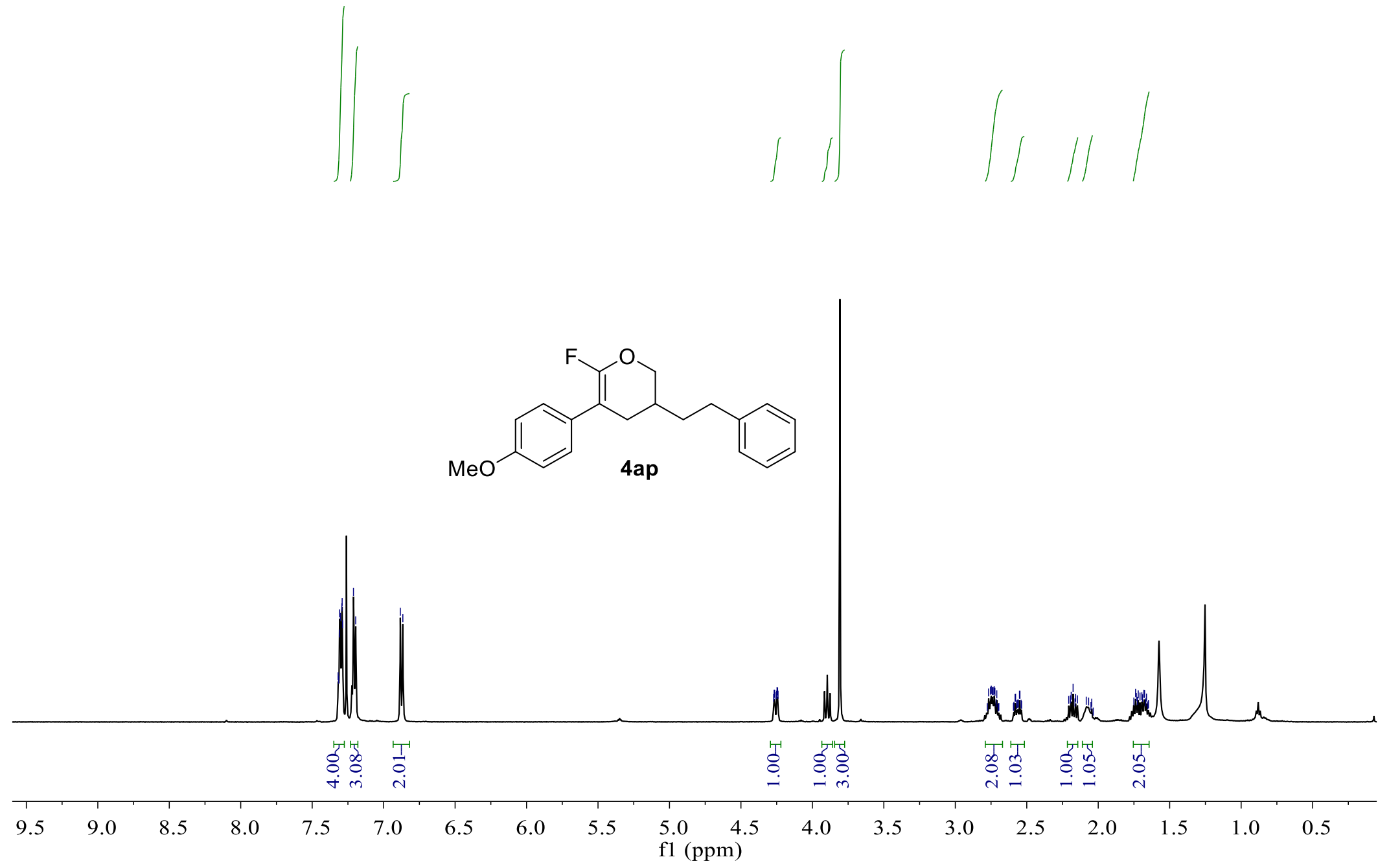

${ }^{13} \mathrm{C}$ NMR-spectrum $\left(126 \mathrm{MHz}, \mathrm{CDCl}_{3}\right)$ of 4 ap 


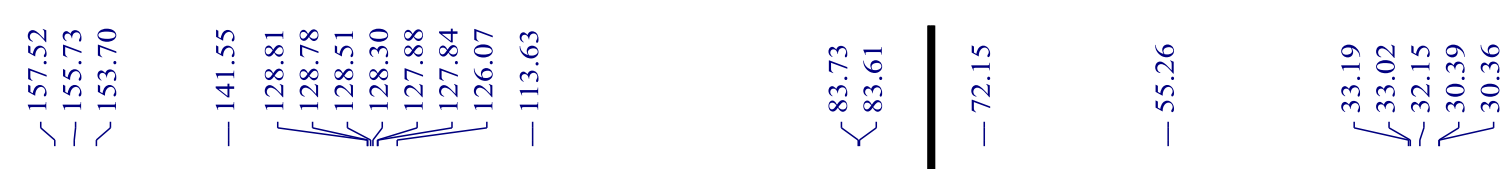
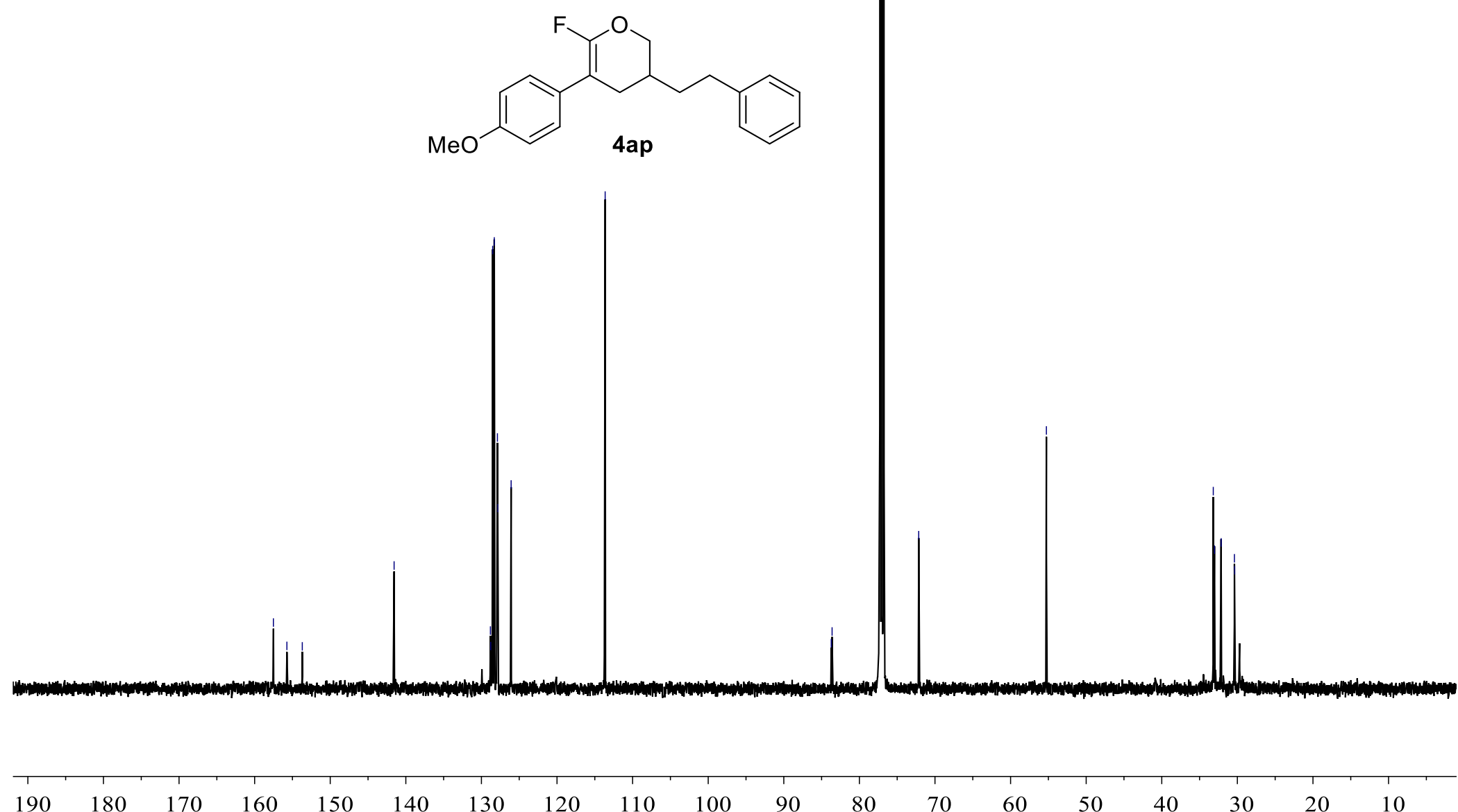

110 10090

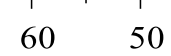

40

${ }^{19} \mathrm{~F}$ NMR-spectrum (471 MHz, $\mathrm{CDCl}_{3}$ ) of 4 ap 


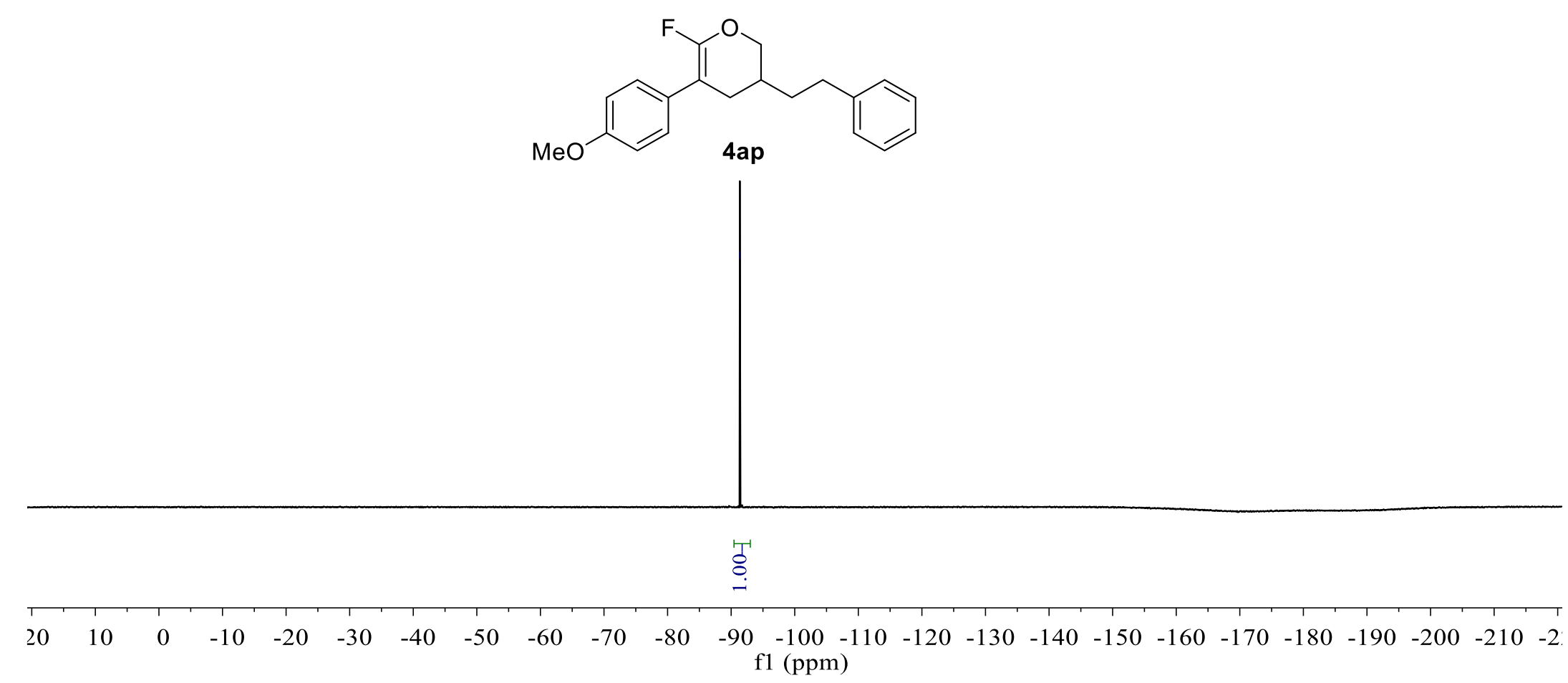

${ }^{1} \mathrm{H}$ NMR-spectrum $\left(400 \mathrm{MHz}, \mathrm{CDCl}_{3}\right)$ of $\mathbf{4 a q}$ 


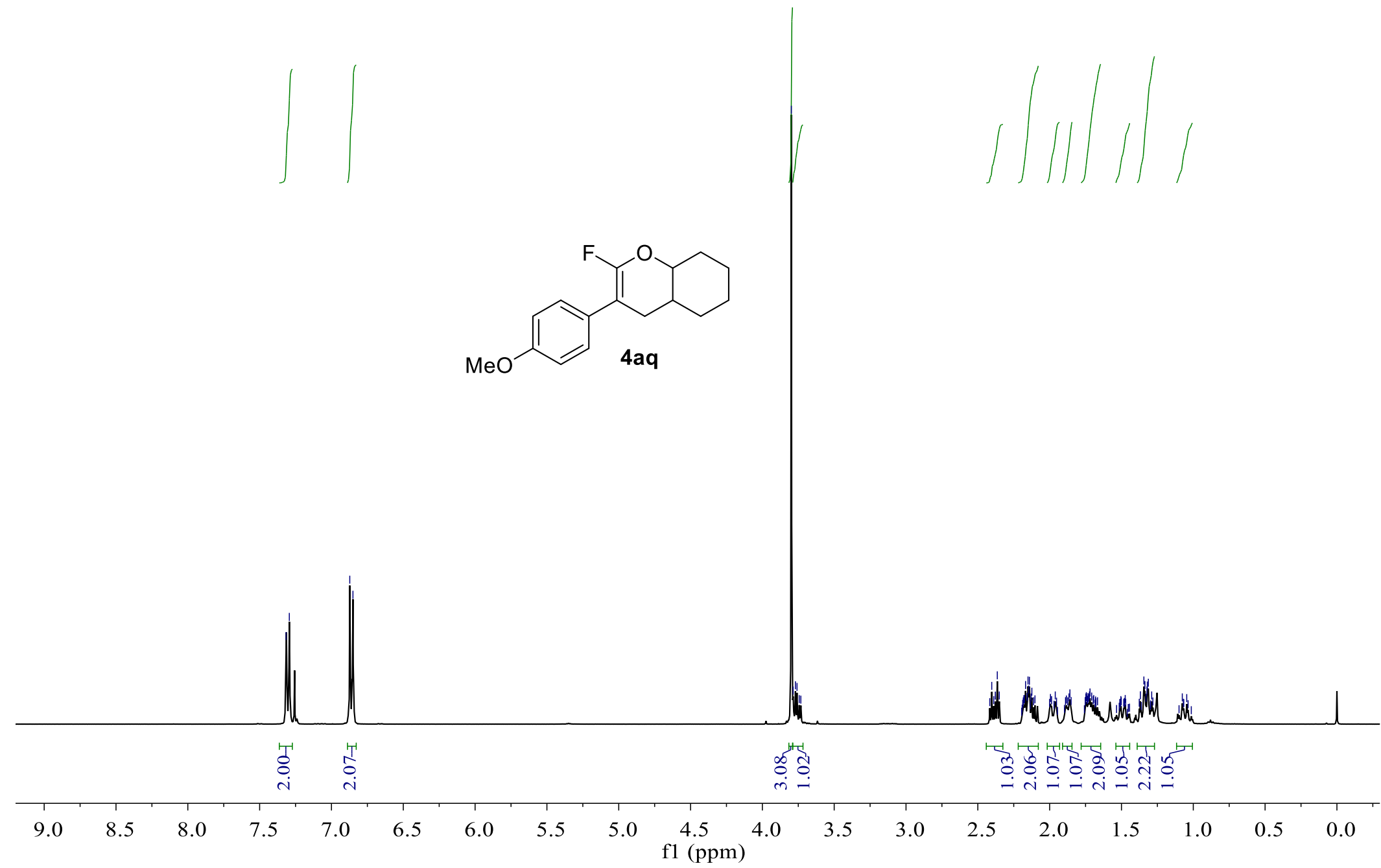


${ }^{13} \mathrm{C}$ NMR-spectrum (101 MHz, $\mathrm{CDCl}_{3}$ ) of $\mathbf{4 a q}$

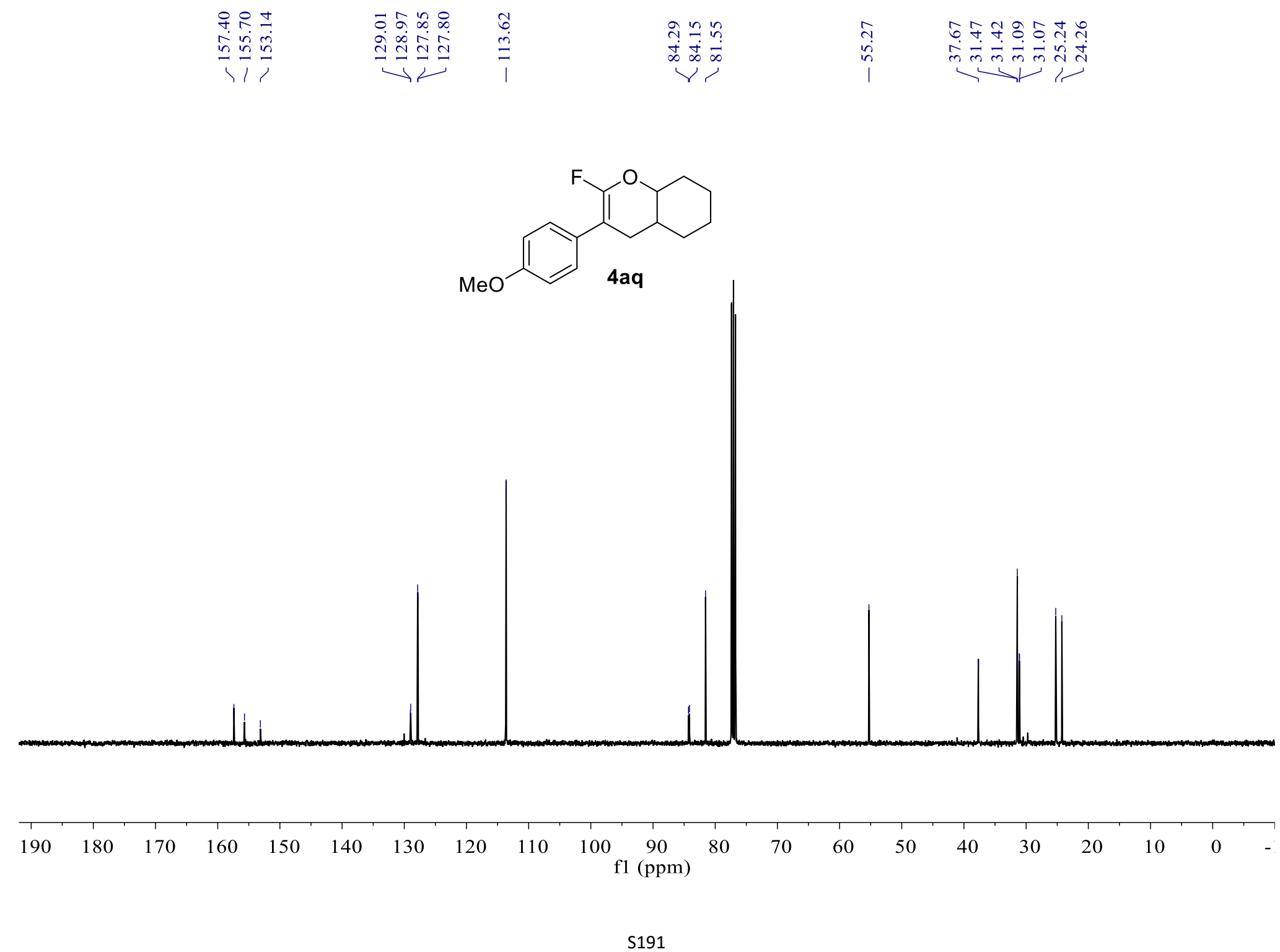


${ }^{19}$ F NMR-spectrum (376 MHz, $\mathrm{CDCl}_{3}$ ) of $\mathbf{4 a q}$

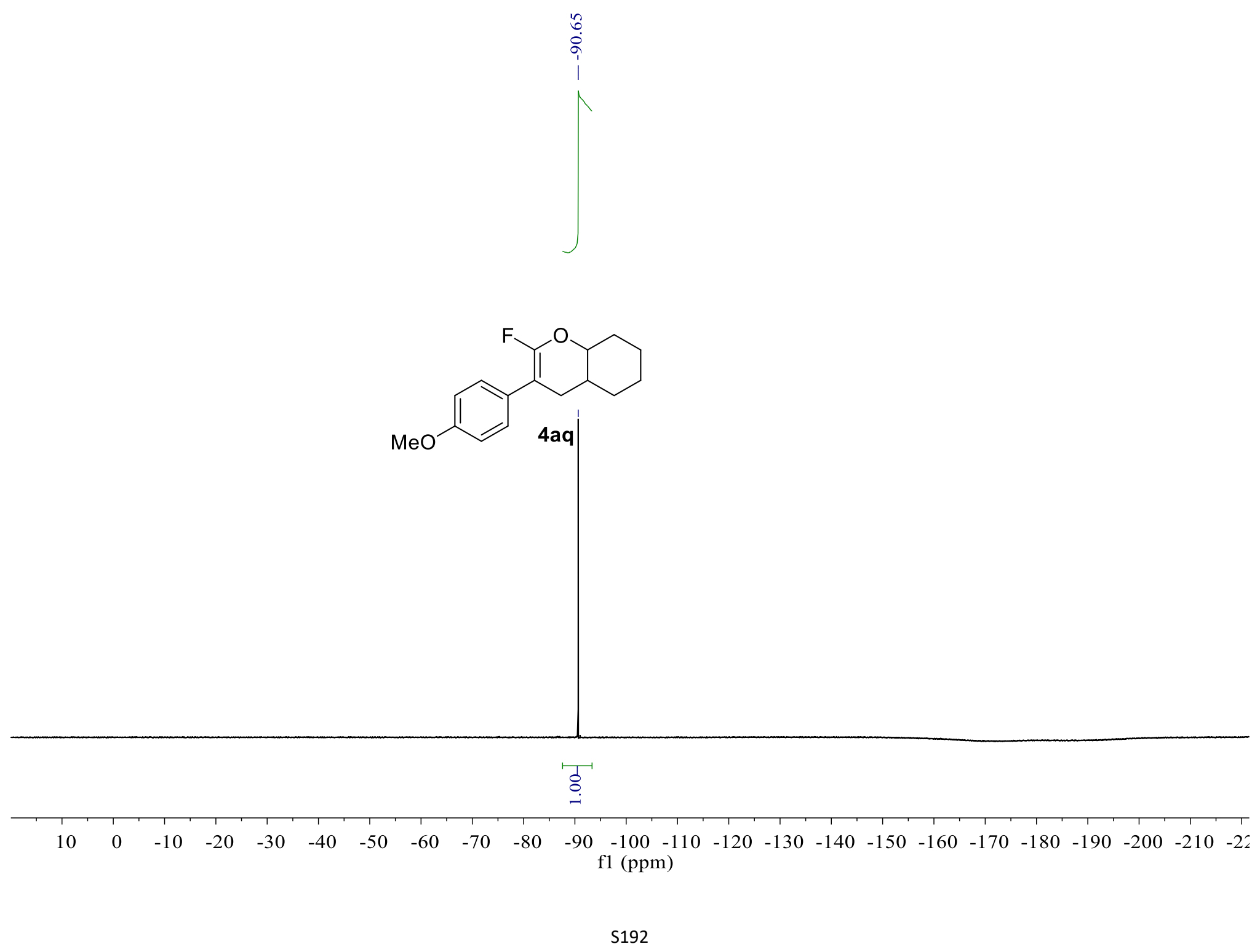




\section{HPLC Data and Chromatograms}
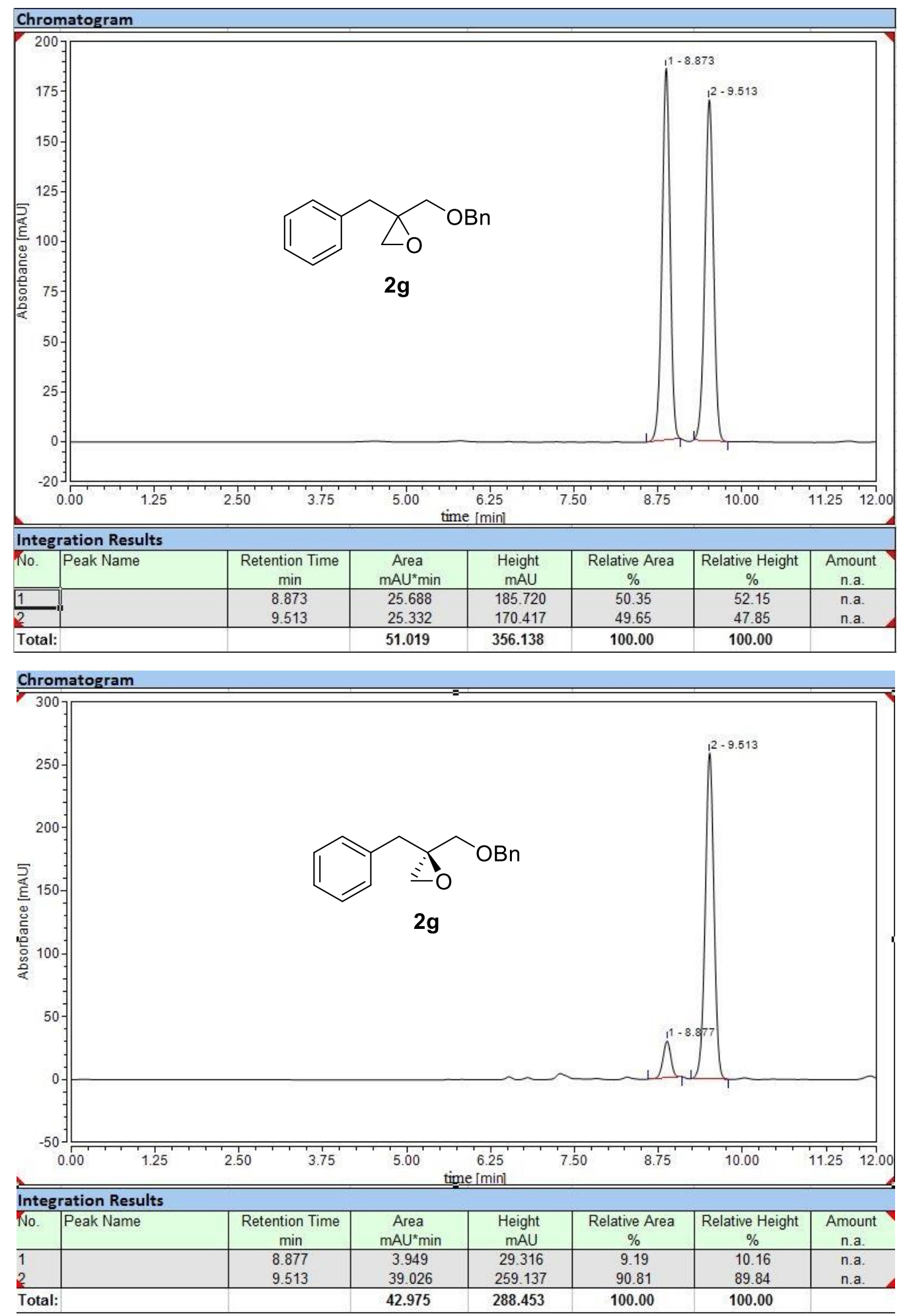

HPLC (ChiralpakIA): $\mathrm{t}_{\mathrm{R}}=8.8$ (minor), 9.5 (major)

Condition: $95: 5 \mathrm{n}-\mathrm{Hexane}: i-\mathrm{PrOH}$, flow rate $0.5 \mathrm{~mL} / \mathrm{min}, 25^{\circ} \mathrm{C}$. 
Recovered epoxide $\mathbf{2 g}$

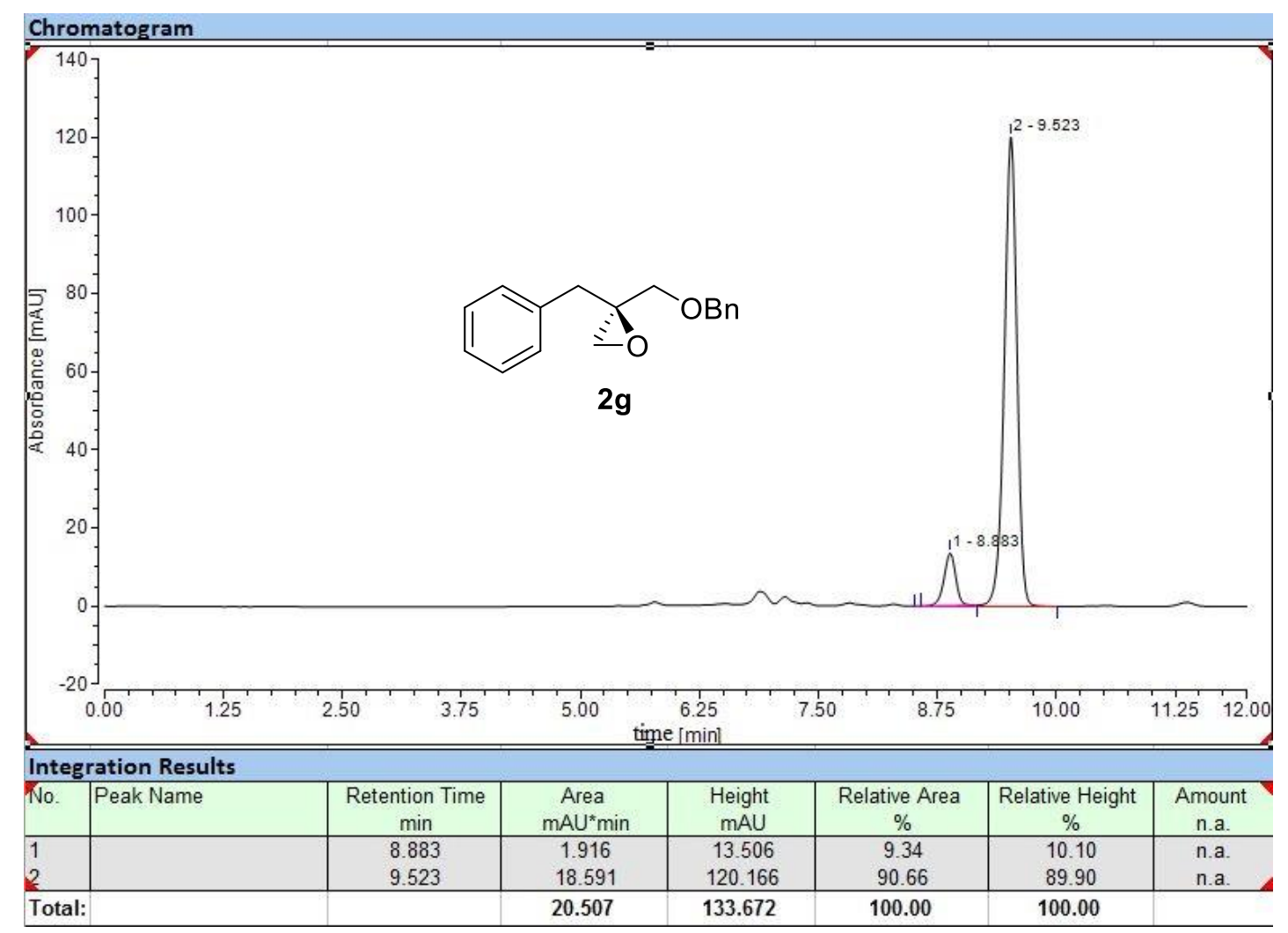

HPLC (ChiralpakIA): $\mathrm{t}_{\mathrm{R}}=8.8$ (minor), 9.5 (major)

Condition: $95: 5 \mathrm{n}-\mathrm{Hexane}: i-\mathrm{PrOH}$, flow rate $0.5 \mathrm{~mL} / \mathrm{min}, 25^{\circ} \mathrm{C}$. 
Cross-Coupling Product Starting from Racemic Epoxide 2g

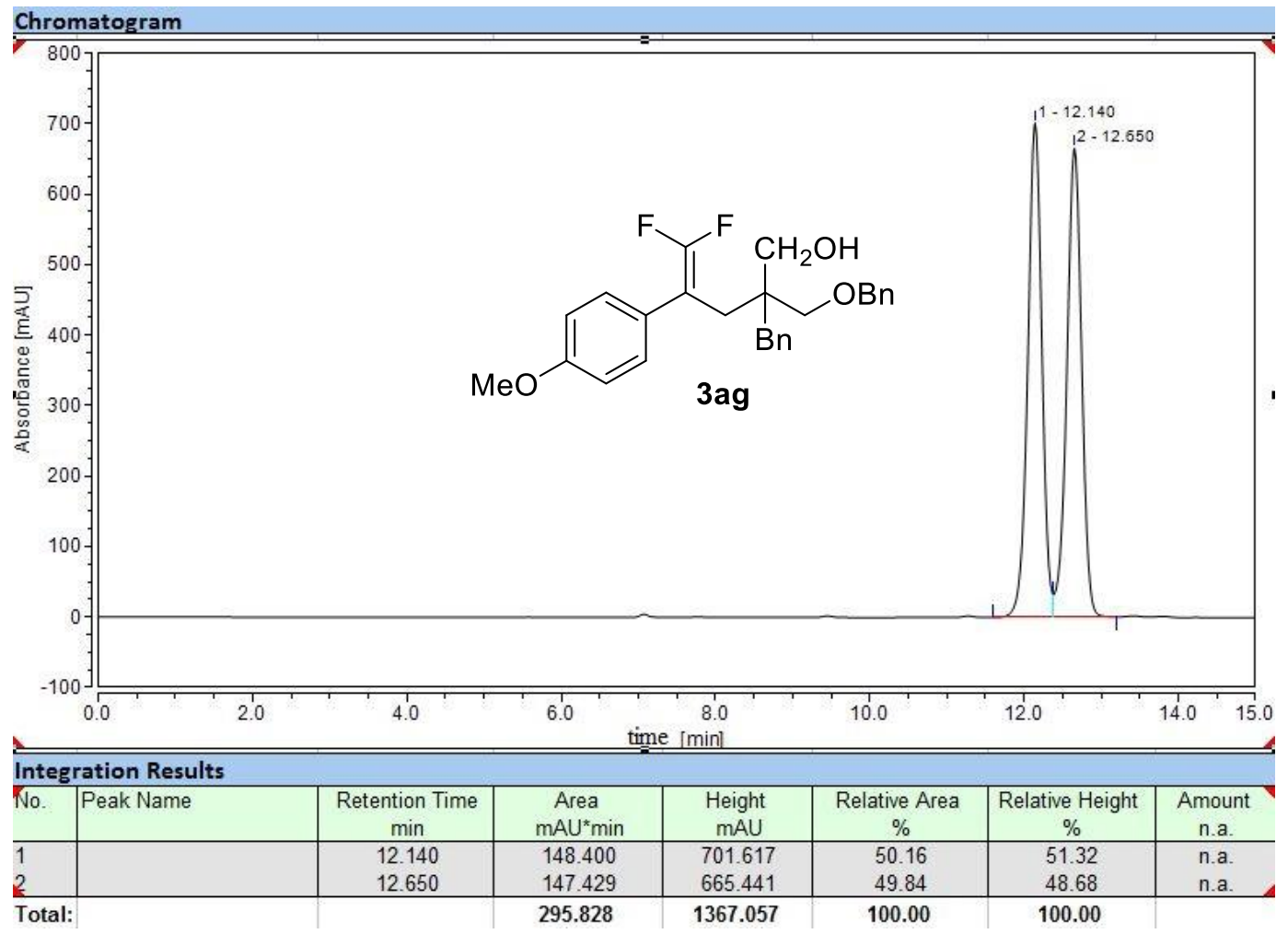

Cross-Coupling Product Starting from Enantioenriched Epoxide 2g

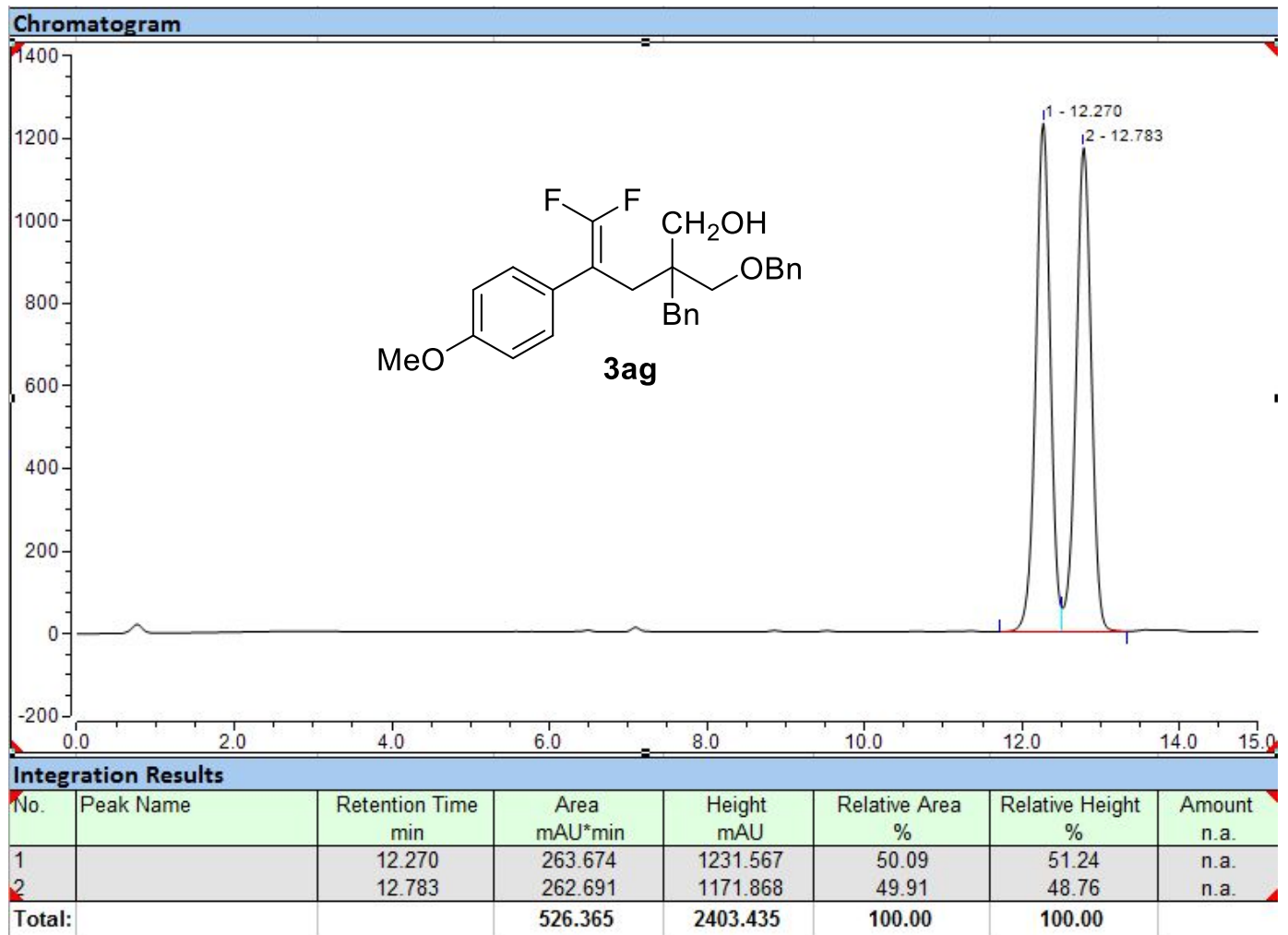

HPLC (ChiralpakIA): $\mathrm{t}_{\mathrm{R}}=12.1$ (minor), 12.6 (major)

Condition: $95: 5 \mathrm{n}-\mathrm{Hexane}: \mathrm{i}-\mathrm{PrOH}$, flow rate $0.5 \mathrm{~mL} / \mathrm{min}, 25^{\circ} \mathrm{C}$. 